\title{
Plasmonic Properties of Supported Silver Nanocrystals: Tuning and Anisotropy
}

\author{
by \\ Adam J. H. Bottomley \\ Doctor of Philosophy \\ in \\ Chemistry \\ Carleton University \\ Ottawa, Ontario \\ (C) 2016 \\ Adam Bottomley
}

A thesis submitted to the Faculty of Graduate and Postdoctoral Affairs in partial fulfillment of the requirements for the degree of 


\section{Abstract}

Silver nanoparticles support strong localized surface plasmon resonances (LSPRs) in the visible region of the spectrum affording them great potential for refractive index sensing, molecular sensing, Raman and fluorescent signal enhancement, and fabrication of optical materials. Characterization of the effects on the plasmonic properties of the particles in terms of size, shape, and anisotropy in their local environment, and how these factors are related to intrinsic properties such as absorption, scattering, and transmission of incident radiation is required for the rational design of materials.

This work focuses on the optical properties of ensembles of silver nanocubes (AgNCs) supported by dielectric materials. AgNCs support multiple plasmon resonances in the ultraviolet-visible region of the spectrum which are strongly influenced by anisotropy in their local environment. Nanocrystals of various sizes studied in this work were synthesized via the polyol method and dispersed as Langmuir films for transfer to solid substrates. Ultraviolet-visible spectroscopy was employed to monitor the peak positions and optical properties associated with the LSPR modes present in these systems.

The peak positions, range, refractive index sensitivities (RIS), and figures of merit (FOM) were determined for observable modes in colloidal and contrasted with those of solid supported AgNCs. Modes present in colloidal AgNCs cannot be tuned independently while some modes present in solid supported AgNC allow for independent tuning by varying the anisotropy of the surrounding environment selectively. Similarly the effects of angle of incidence and polarization on transmission, scattering, and absorption of incident radiation is quantified for solid supported AgNCs as functions of size and refractive index of the solid support. 


\section{Preface}

This preface provides full bibliographical details for each article included in this thesis, as well as whether the article is reproduced in whole or in part. Use of copyrighted material is likewise acknowledged here. When citing material from this thesis, please cite the article relevant to the chapter, if the chapter is based on a publication.

Pursuant to the Integrated Thesis policy of Carleton University, the "supervisor" (Anatoli Ianoul) and the "student" (Adam Bottomley) confirm that the student was fully involved in setting up and conducting the research, obtaining data and analyzing results, as well as preparing and writing the material presented in the co-authored article(s) integrated in the thesis. Additionally, the supervisor confirms the information provided by the student in this preface.

\section{Chapter 1}

Figure 1.1 is reproduced with permission from the Nature Publishing Group, 2007. Figure 1.3 is reproduced with permission from Daniel Prezgot (Carleton Unviersity), 2014. Figure 1.4 is reproduced with permission from the American Chemical Society, 2011. Figure 1.5 is reproduced with permission from IOP Publishing, 2010.

Figure 1.6 is reproduced with permission from the American Chemical Society, 2004. Figure 1.7 is reproduced with permission from the American Chemical Society, 2009. Figure 1.8 is reproduced with permission from the American Chemical Society, 2011. Figure 1.9 is reproduced with permission from the American Chemical Society, 2013.

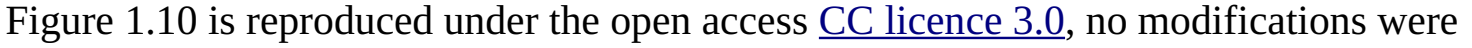
made to the figure.

Figure 1.11 is reproduced with permission from the Nature Publishing Group, 2015. Figure 1.12 is reproduced with permission from John Wiley and Sons, 2005.

\section{Chapter 2}

Bottomley, A., Prezgot, D. and Ianoul, A., Plasmonic properties of silver nanocube monolayers on high refractive index substrates. Applied Physics A 2012, 109, 869-872.

The article is wholly reproduced and edited for formatting and clarity of presentation. Adam Bottomley performed the majority of experimental work with the assistance of Daniel Prezgot on the synthesis of silver nanocubes and formation and characterization of monolayers. The article was written by Anatoli Ianoul.

\section{Chapter 3}

Bottomley, A. and Ianoul, A., Improved Refractive-Index Sensitivity of Silver-Nanocube Monolayers on Silicon Films. ChemPhysChem 2011, 12, 2912-2914.

The article is wholly reproduced and edited for formatting and clarity of presentation. Adam Bottomley performed all of the experimental work. The article was written by Anatoli Ianoul. 


\section{Chapter 4}

Ahamad, N., Bottomley, A. and Ianoul, A., Optimizing Refractive Index Sensitivity of Supported Silver Nanocube Monolayers. J. Phys. Chem. C 2012, 116, 185-192.

The article is wholly reproduced and edited for formatting and clarity of presentation. Adam Bottomley performed all of the work related to 40 and 80 nanometer cubes including synthesis, and fabrication and characterization of monolayers. Nur Ahamad performed the experimental work associated with 130 nanometer cubes. This article was written by Nur Ahamad and Adam Bottomley.

\section{Chapter 5}

Bottomley, A., Prezgot, D., Staff, A. and Ianoul, A., Fine tuning of plasmonic properties of monolayers of weakly interacting silver nanocubes on thin silicon films. Nanoscale 2012, 4, 6374-6382.

The article is wholly reproduced and edited for formatting and clarity of presentation. Adam Bottomley performed the majority of work with assistance Daniel Prezgot and Alyssa Staff related to nanocube synthesis, monolayer formation and characterization of final materials. SERS experiments were performed by Anatoli Ianoul. This article was written by Anatoli Ianoul and Adam Bottomley.

\section{Chapter 6}

Bottomley, A. and Ianoul, A., Reflection and Absorption Spectra of Silver Nanocubes on a Dielectric Substrate: Anisotropy, Angle, and Polarization Dependencies. J. Phys. Chem. C 2014, 27509-27515.

The article is wholly reproduced and edited for formatting and clarity of presentation. Adam Bottomley performed all of the experimental work. The article was written by Anatoli Ianoul and Adam Bottomley.

\section{Chapter 7}

Bottomley, A., Prezgot, D., Coyle, P. J., and Ianoul, A., Dynamics of nanocubes embedding into polymer films investigated via spatially resolved plasmon modes. Nanoscale 2016, 8, 11168-11176.

This article is wholly reproduced and edited for formatting and clarity of presentation. Adam Bottomley performed all of the reported experimental work. Initial exploratory work performed by Adam Bottomley and Jason Coyle. FDTD simulations performed by Daniel Prezgot. The article was written by Adam Bottomley, Daniel Prezgot, and Anatoli Ianoul. 


\section{Acknowledgments}

First and foremost I would like to thank Dr. Anatoli Ianoul for giving me the opportunity and guidance to conduct my research. He taught me not only how to perform and understand research at the academic level, but how to truly become a scientist. His lessons will surely help me throughout the entirety of my career.

I would like to thank the various grad students I worked along side with over the years in the Ianoul lab, Amy Won, Graham Galway, Nur Ahamad, Daniel Prezgot, Devin O'Neill, Alyssa Staff, Grace Idiong, and Emma Jorgenson, for their insightful conversations and welcome distractions. I would also like to thank the long list if undergraduate researchers and summer students who passed through the Ianoul lab during my time here.

I would like to thank the Natural Sciences and Engineering Research Council of Canada (NSERC), SPIE the International Society for Optics and Phonics, and Carleton University for funding me during my studies.

I would like to acknowledge the people in the department of chemistry and their support. In particular the help by Dr. Jainqun Wang with electron microscopy, Jim Logan for his help with building and designing various parts required for my experiments, Sean Barry for saving my academic career several times, and Chantelle Gravelle for helping me stay on track during the final year of my education.

A special thanks to Rob Vandusen, Angie McCormick, and Rick Adams for their help with materials fabrication and characterization in the Carleton University Micro-fabrication Laboratory. 


\section{Table of Contents}

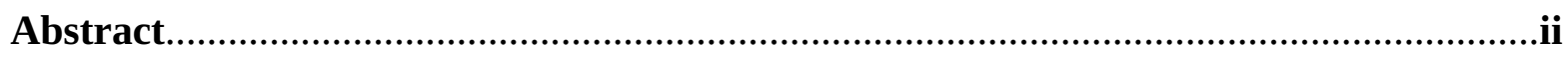

Preface

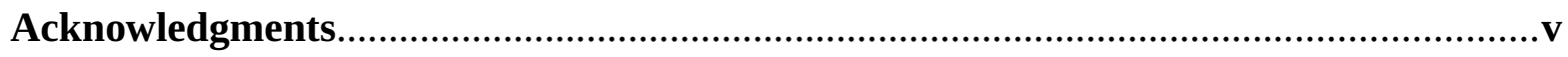

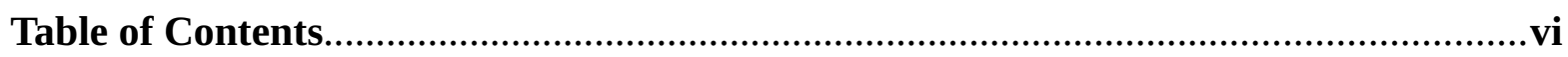

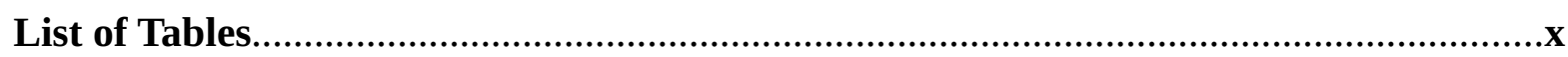

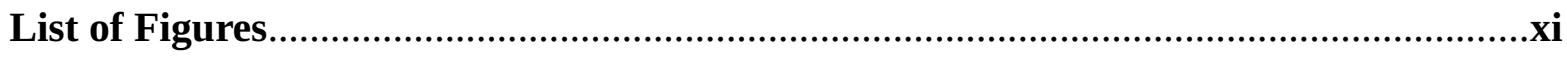

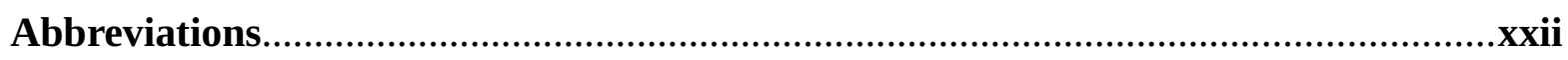

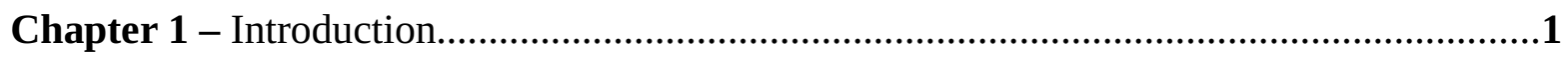

1.1 Historical Development....................................................................................

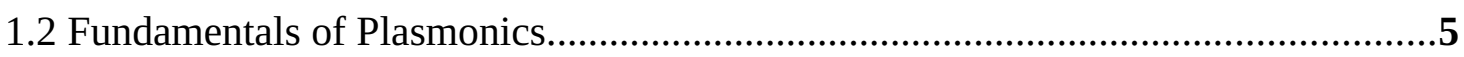

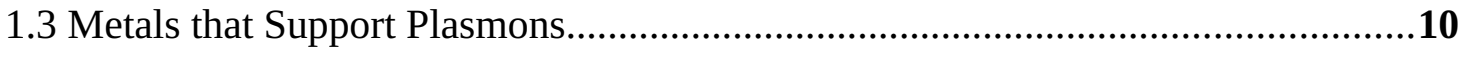

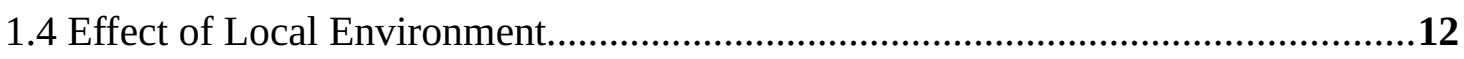

1.4.1 Size and Shape

1.4.2 Inter-particle Coupling............................................................................13

1.4.3 The Plasmon Hybridization Model...............................................................14

1.4.4 Higher Order and Dark Modes...................................................................15

1.4.5 Substrate Interactions...........................................................................16

1.4.6 Fano Interferences.....................................................................................18

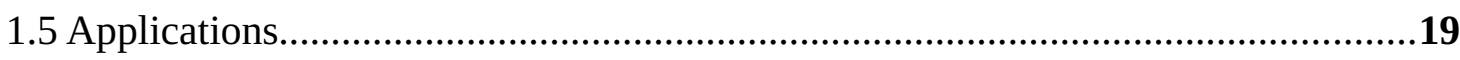

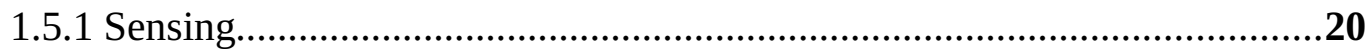

1.5.2 Field Enhanced Spectroscopy..................................................................21

1.5.3 Nanorulers............................................................................................22 
1.5.4 Thermoplasmonics................................................................................23

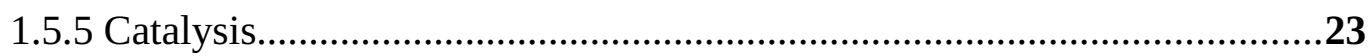

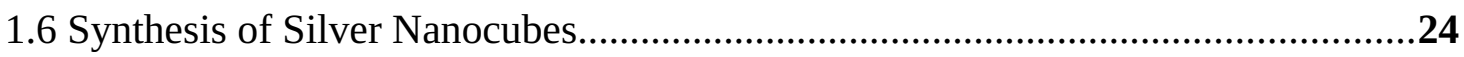

1.7 Rationale

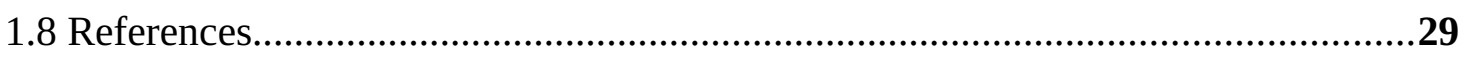

Chapter 2 - Plasmonic properties of silver nanocube monolayers on high refractive index

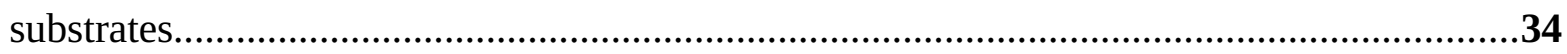

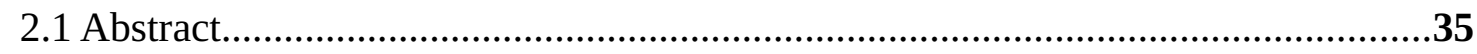

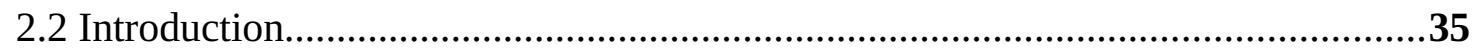

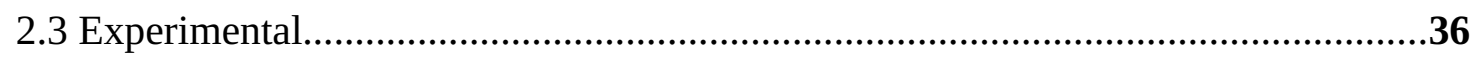

2.4 Results and Discussion...........................................................................................39

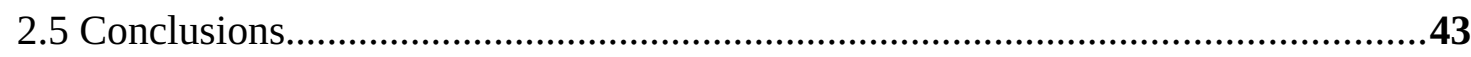

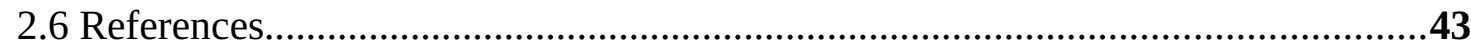

Chapter 3 - Improved Refractive Index Sensitivity of Silver Nanocube Monolayers on

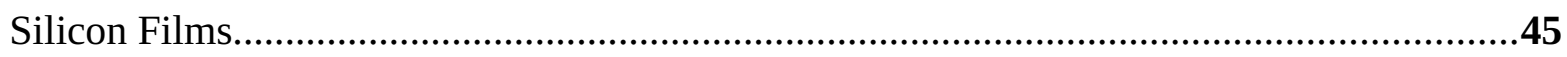

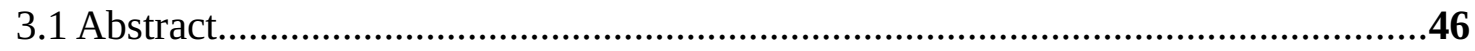

3.2 Results and Discussion.....................................................................................46

3.3 Experimental................................................................................................51

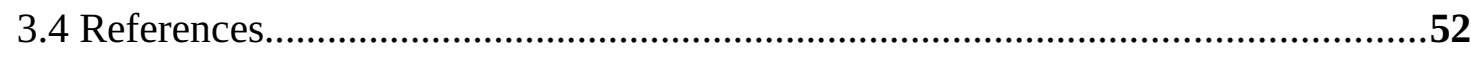

Chapter 4 - Optimizing Refractive Index Sensitivity of Supported Silver Nanocube

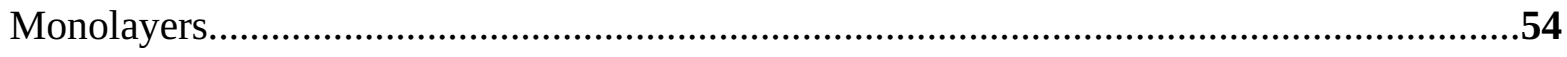

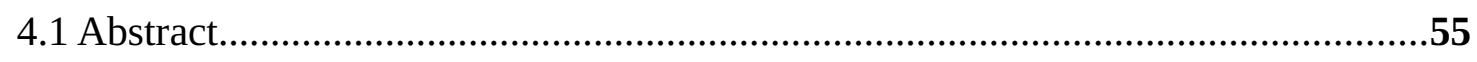

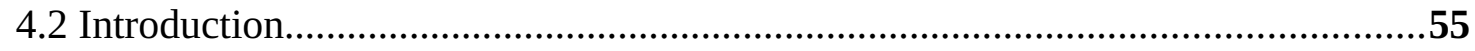

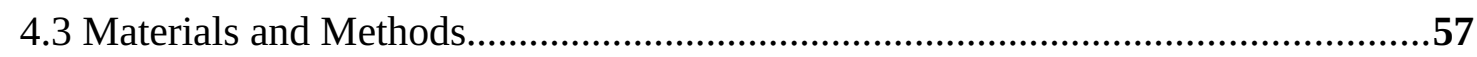




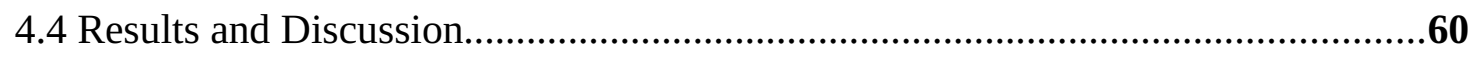

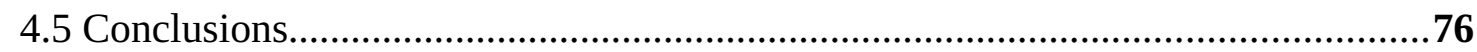

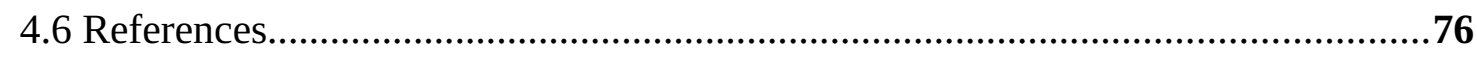

Chapter 5 - Fine tuning of plasmonic properties of monolayers of weakly interacting silver

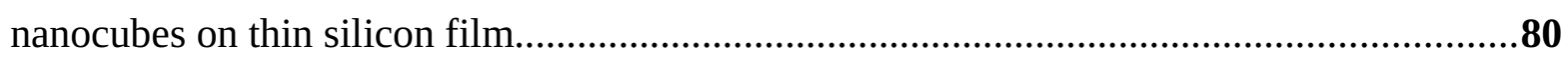

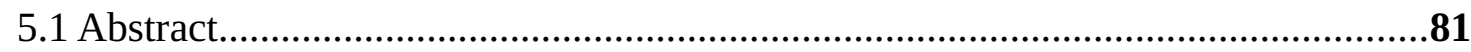

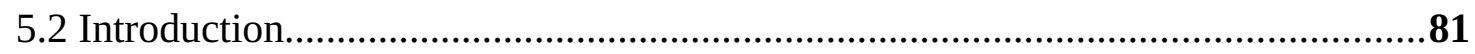

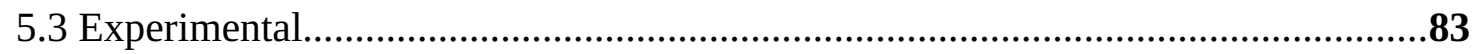

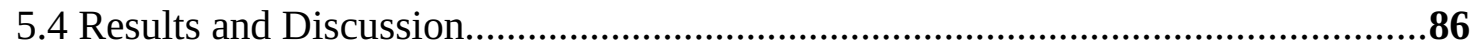

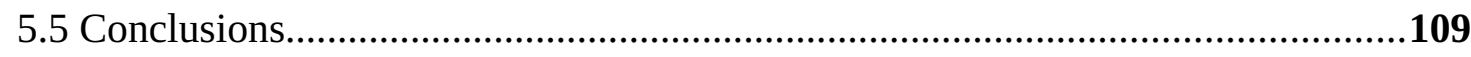

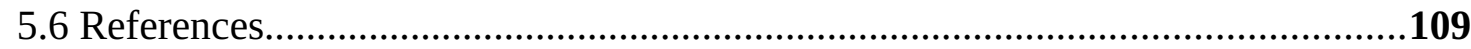

Chapter 6 - Reflection and Absorption Spectra of Silver Nanocubes on a Dielectric

Substrate: Anisotropy, Angle, and Polarization Dependencies...........................................112

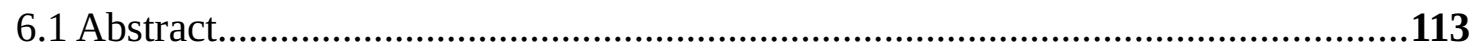

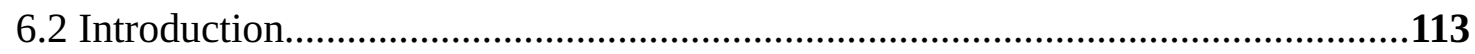

6.3 Materials and Methods......................................................................................115

6.4 Results and Discussion............................................................................118

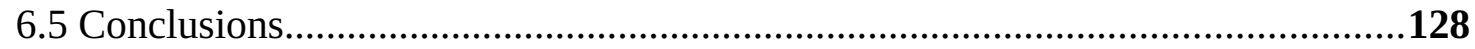

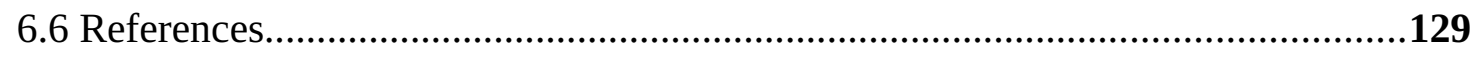

Chapter 7 - Dynamics of nanocubes embedding into polymer films investigated via spatially resolved plasmon modes....................................................................................132

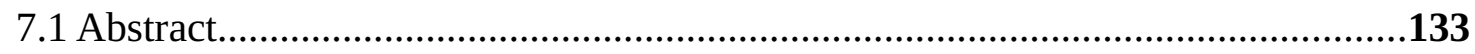

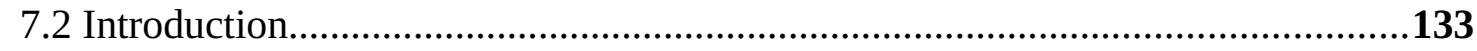

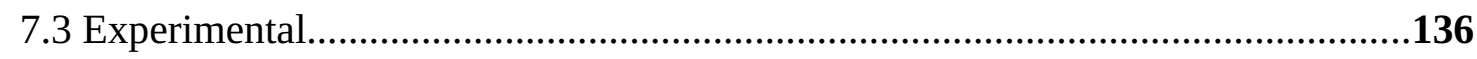




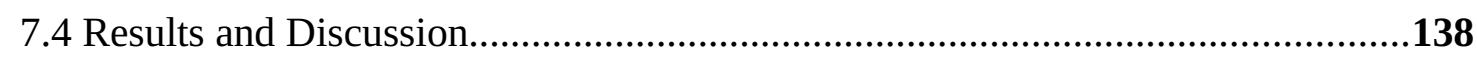

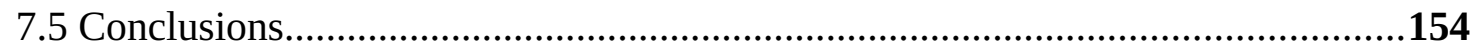

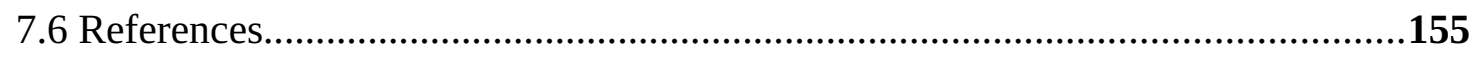

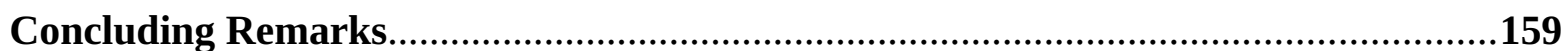

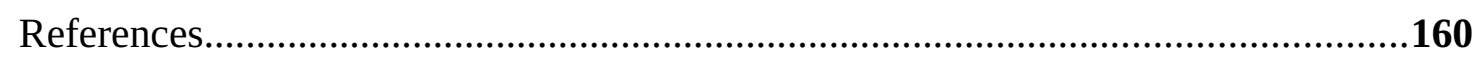




\section{List of Tables}

Table 4.1 Refractive Index Sensitivities of Silver Nanocubes in Suspension.........................64 Table 4.2 Refractive Index Sensitivities of Supported Silver Nanocube Monolayers on Glass

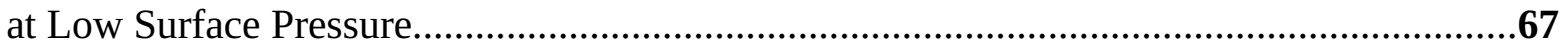

Table 5.1 Refractive index sensitivities of monolayers of 35-40nm silver nanocubes on silicon. 98

Table 5.2 Refractive index sensitivities of monolayers of $40-45 \mathrm{~nm}$ silver nanocubes on silicon. 99

Table 5.3 Refractive index sensitivities of monolayers of $75-80 \mathrm{~nm}$ silver nanocubes on silicon .99

Table 5.4 Refractive index sensitivities of monolayers of $75-80 \mathrm{~nm}$ silver nanocubes on silicon. 


\section{List of Figures}

Figure 1.1 Lycurgus Cup, (British Museum, Fourth Century AD), This Roman cup contains gold nanoparticles making it dichroic. Reflected light gives it a green colour, but it attains a ruby red when viewed with transmitted light. .2

Figure 1.2 Appearance of the term 'Surface Plasmon' in scientific literature. SciFinder Scholar@. .5

Figure 1.3 Schematic illustration of a dipolar localized surface plasmon. Conducting electrons are displaced by the electric component of resonant frequency EM radiation. This leads to a strong optical extinction as well as an enhanced electric field surrounding the particle itself. .9

Figure 1.4 Plot of the real $\varepsilon_{\mathrm{r}}(\mathrm{A})$, and imaginary $\varepsilon_{\mathrm{i}}(\mathrm{B})$ components of the dielectric functions for silver, gold, and silicon in the visible spectrum.

Figure 1.5 Periodic table of the elements showing the highest quality factor attainable for each element. Frequencies are in eV. .11

Figure 1.6 Schematic illustrating plasmon hybridization in a nanoparticle dimer. The plasmons in each particle interact with each other in both a constructively and deconstructively in a similar manner to bonding and anti-bonding orbitals in molecules. This interaction generates a bright coupling mode. Interactions between modes in adjacent particles lowers their energy causing red shifts in their peak positions. .14

Figure 1.7 Schematic showing the polarization dependent substrate interactions between a plasmonic nanoparticle and a dielectric substrate. 16

Figure 1.8 (a) Simulated scattering spectra from a cube in vacuum (black line) and a cube on a glass substrate (red line). (b) and (c) correspond to the electric field amplitude for peaks I 
and II from the red curve respectively

Figure 1.9 Fano interferences caused by hybridization of plasmon modes in an array of 4 gold nanobars as the distance between the two middle bars is varied. (a) Experimental darkfield scattering spectra. (b) FDTD simulated scattering spectra. (c) Plasmon hybridization model calculated spectra. (d) SEM images of the measured arrays

Figure 1.10 Example of a SPR sensor in the Kretschmann configuration. The resonant frequency of the SPP at a fixed angle will redshift as more target material is bound to the surface opposite of illumination. 20

Figure 1.11 Schematic representation depicting the events that lead to thermal generation and dissipation beginning with the initial photoexitation of a plasmon. 23

Figure 1.12 Schematic showing the fate of initial silver ions and illustrating the pathways towards different morphologies based on crystallinity of seeds produced in the reaction.....25

Figure 2.1 Supported silver nanocube monolayer prepared by Langmuir Blodgett technique: (a) AFM topography and (b) Fast Fourier transform (FFT) images indicate the size (55nm) and the average interparticle distance (130nm); (c) extinction spectra of supported silver nanocube monolayers indicate strong dependence of the dipolar (D) but not the quadrupolar (Q) mode on the substrate refractive index. 40

Figure 2.2 Solution refractive index sensing using nanocube monolayers deposited on glass and on a $7 \mathrm{~nm}$ thick silicon film. Inset shows solution refractive index dependencies of the dipolar (D) and quadrupolar (Q) peaks. 41

Figure 3.1 UV/Vis spectra of the Ag nanocubes: A) Monolayer deposited on a thin Si film.

B) Monolayer deposited on quartz C) Suspension in EtOH. 47

Figure 3.2 UV/Vis spectra of a monolayer of Ag nanocubes deposited on a Si film immersed in: A) water, and B) ethylene glycol. The solid curves (-) represent the experimental 
spectra, the dashed curves (----) show the results of the spectral deconvolution, and the dotted curves $(\cdots \bullet)$ represent individual peaks

Figure 3.3 Plasmon shifts for dipolar (peak 3) and quadrupolar (peak 2) resonances for a monolayer of silver nanocubes deposited on a thin Si film.

50

Figure 4.1 Atomic force microscopy of supported nanocube substrate prepared by Langmuir monolayer technique at low $(0.1-1 \mathrm{mN} / \mathrm{m}, 15 \mathrm{mN} / \mathrm{m}$ for $130 \mathrm{~nm}$ cubes) surface pressure. Histograms show height distribution in the samples

Figure 4.2 Extinction spectra of colloidal suspensions in different water/ethylene glycol solutions for (A) 40, (B) 80, and (C) 130nm nanocubes. Spectra were measured in water (lowest spectra in series), followed by water:EG 4:1, ethanol (40 and 80nm only), water:EG 3:2, 2:3, 1:4, and pure EG .63

Figure 4.3 Plasmonic shifts as a function of the refractive index for silver nanocube suspensions. 65

Figure 4.4 Extinction spectra of supported nanocube monolayers in air (lowest spectra in series) and immersed in different water/ethylene glycol solutions for (A) $40 \mathrm{~nm}$ on a glass substrate, (B) $40 \mathrm{~nm}$ on a glass substrates coated with $5 \mathrm{~nm} \mathrm{TiO}_{2}$ film, (C) $80 \mathrm{~nm}$, and (D) $130 \mathrm{~nm}$ nanocubes. Spectra were measured in water (first spectra in series), followed by water:EG 4:1, water:EG 3:2, 2:3, 1:4, and pure EG. .66

Figure 4.5 Plasmonic shifts as a function of the refractive index for supported silver nanocubes. 68

Figure 4.6 Left: UV-vis spectra of supported 80nm nanocubes on different substrates: quartz, sapphire, glass slide coated with $5 \mathrm{~nm} \mathrm{TiO}_{2}$ film, glass slide coated with $5 \mathrm{~nm}$ of silicon. Right: Plasmon shifts plotted vs. the refractive index of a substrate. 70

Figure 4.7 UV-vis spectra of supported 40nm nanocubes (A) and irregular-shaped 
nanoparticles (B) on different substrates: (1) quartz, (2) glass, (3) sapphire, (4) glass slide with coated with $5 \mathrm{~nm} \mathrm{TiO}_{2}$ film, (5) glass slide coated with $5 \mathrm{~nm}$ of silicon, (6) spectra of suspensions in ethanol. 72

Figure 4.8 Plasmon shift of the dipolar (diamonds) and quadrupolar (squares, cubes only) bands in extinction spectra of supported 40nm Ag nanocubes (open symbols) and irregular Ag nanoparticles (filled symbols) as a function of support refractive index .73

Figure 4.9 Angular dependence of UV-vis extinction spectra for 40nm nanocubes deposited on different substrates. .75

Figure 5.1 AFM topography images of a monolayer of 35-40nm silver nanocubes-DOPC deposited by the Langmuir technique on a $15 \mathrm{~nm}$ thick silicon film at (A) $1.5 \mathrm{mN} / \mathrm{m}$ and (B) $4.5 \mathrm{mN} / \mathrm{m}$. Insets show the corresponding fast Fourier transformation (FFT) images. Two concentric rings in FFT of the $1.5 \mathrm{mN} / \mathrm{m}$ sample indicate the short range order with a nearest neighbour distance (center-to-center) of $\sim 120 \mathrm{~nm}$ and nanocube size of $\sim 40 \mathrm{~nm}$. The short range order disappears at $4.5 \mathrm{mN} / \mathrm{m}$ according to the FFT image. (C) Topographical analysis of the monolayer deposited at $1.5 \mathrm{mN} / \mathrm{m}$ indicating the average height of the nanocubes.....87 Figure 5.2 Left column: AFM topography images of monolayers of 35-40nm silver nanocubes/DOPC deposited on a 15nm thick silicon film at various surface pressures, as indicated. Right column: corresponding Fast Fourier transformation images corresponding to the images on the left side. Concentric circles indicate the presence of short range order (middle to middle, the smallest circle), and the particle size (second circle) respectively. The third concentric circle indicates the separation between the particles (side to side). Short range order disappears at high surface pressure of $4.5 \mathrm{mN} / \mathrm{m}$. .87

Figure 5.3 UV-vis extinction spectra for monolayers of 35-40nm silver nanocube/ DOPC deposited at various surface pressures on a $15 \mathrm{~nm}$ silicon film (left) or glass slide 
(corresponding to figure 5.2 images). Deposition was performed simultaneously onto glass and silicon sides of the substrate. The strong dipole-dipole coupling peak becomes dominant at high surface pressure of $4.5 \mathrm{mN} / \mathrm{m}$. The photo shows s slide with silicon film on the left hand side. The bottom part of the slide is coated with the nanocube/DOPC monolayer .89

Figure 5.4 Extinction spectra of silver nanocubes in ethanol (black lines) and silver nanocube-DOPC monolayers deposited on silicon films of 0 (glass, blue lines), 10 (green), 15 (orange) and 25nm (red) thickness for (A) 35-40nm cubes; (B) 40-45nm cubes; (C) 7580nm cubes, and (D) 80-85nm cubes. Blue squares indicate the positions of the quadrupolar peaks and red rhombs - dipolar peaks. Graphs at the top of each panel indicate the relative quadrupolar (Q) or dipolar (D) integral extinction intensities as a function of Si film thickness. Results of spectral fitting are presented in Fig. 5.5.........................................90

Figure 5.5 Results of extinction spectra deconvolution for silver nanocubes in ethanol solution (the top most spectra) and for nanocube/ DOPC monolayers deposited on different substrates in air. Series in columns correspond to samples A, B, C, and D (from left to right) in Figure 5.4. Red thick curves represent experimental data. Dark blue thick curves represent results of spectral fitting. Blue and red peaks correspond to quadrupolar and dipolar modes respectively. Silicon film thickness is indicated. 91

Figure 5.6 Position of the dipolar plasmon mode as a function of silicon film thickness for four different monolayers. .92

Figure 5.7 (A) Angle dependence of extinction spectra for the 40-45nm nanocube monolayer deposited on a $\sim 15 \mathrm{~nm}$ Si film for s- and p- polarized light. (B) Relative quadrupolar (Q) or dipolar (D) integral extinction intensities as a function of angle for ppolarized light. .94

Figure 5.8 Refractive index sensitivities of supported silver nanocube monolayers on Si 
films. (A) 35-40nm cubes, (B) 40-45nm cubes, (C) 75-80nm cubes, and (D) $80-85 \mathrm{~nm}$ cubes. Black lines represent extinction spectra in air; magenta, blue, green, and red in H2Oethylene glycol 1/0, 2/1, 1/2, and 0/1 mixtures respectively. Blue squares indicate the positions of the quadrupolar peaks and red rhombs - dipolar peaks for monolayers immersed in liquids. .95

Figure 5.9 Results of extinction spectra deconvolution for 35-40nm silver nanocubes/DOPC monolayers deposited on different silicon films immersed in water/ethylene glycol mixtures 1/0,2/1, 1/2, and 0/1 volume ratios. Series correspond to the sample A in Figure 5.8. Red thick curves represent experimental data. Dark blue thick curves are the result of spectral fitting. Purple lines show the difference between the fitted and experimental spectra. Blue and red peaks are quadrupolar and dipolar modes respectively. .97

Figure 5.10 Results of extinction spectra deconvolution for 75-80nm silver nanocubes/DOPC monolayers deposited on different silicon films immersed in water/ethylene glycol 1/0, 2/1, 1/2, and 0/1 volume ratios. Series correspond to the sample C in Figure 5.8. Red thick curves represent experimental data. Dark blue thick curves are the result of spectral fitting. Blue and red peaks are quadrupolar and dipolar modes respectively

Figure 5.11 Results of extinction spectra deconvolution for $80-85 \mathrm{~nm}$ silver nanocubes/DOPC monolayers deposited on different silicon films immersed in water/ethylene glycol 1/0, 2/1, 1/2,and 0/1 volume ratios. Series correspond to sample D in Figure 5.8. Red thick curves represent experimental data. Dark blue thick curves are the result of spectral fitting. Blue and red peaks are quadrupolar and dipolar modes respectively

Figure 5.12A Plasmonic shifts as functions of solution refractive index for supported 
nanocube/DOPC monolayers in figure 5.8A (left) and B (right)

Figure 5.12B Plasmonic shifts as functions of solution refractive index for supported nanocube/DOPC monolayers in figure 5.8C(left) and D(right). .102

Figure 5.13 Deconvolution of extinction spectra for the $80-85 \mathrm{~nm}$ nanocube sample deposited on a 10nm silicon film in water-ethylene glycol 1/0, 2/1, 1/2, and 0/1 mixtures. Red thick curves represent experimental data. Dark blue thick curves are the result of spectral fitting. Blue and red peaks are quadrupolar and dipolar modes respectively. The top black spectrum is measured in air 103

Figure 5.14 Plasmonic shifts as functions of refractive index for supported nanocube-DOPC monolayers for peaks shown in Fig. 5.13. 104 Figure 5.15 SERS spectra of rhodamine 6G on glass (blue curves) and a 7nm silicon film (red curves) at different excitation wavelengths 458, 488, 514, 568 and 647nm. Extinction spectra for nanocube-lipid monolayers on glass (blue dashed curve) and silicon (red dashed curve) are presented. Extinction and fluorescence spectra for Rh6G are shown as black dotted lines. SERS spectra were normalized by using the intensity of the $1508 \mathrm{~cm}^{-1}$ band of Rh6G on glass at different wavelengths.

Figure 5.16 SERS spectra of Rhodamine 6G $(1 \mu \mathrm{M})$ deposited on a monolayer of $\sim 60 \mathrm{~nm}$ silver nanocubes/DOPC on glass (blue) and 7nm silicon film (red). The spectra were obtained by averaging spectra from 9 different areas of the sample. Accumulation time in each point was 60 seconds. Excitation wavelength used was 458nm with laser power below $1 \mathrm{~mW}$ .105

Figure 5.17 SERS enhancement factor as a function of excitation wavelength for spectra shown in Fig. 5.15. 106

Figure 5.18 Time dependence of the rhodamine 6G SERS spectrum on glass (blue) and a 
$7 \mathrm{~nm}$ silicon film (red). The excitation wavelength was $488 \mathrm{~nm}$ and power was $50 \mathrm{~mW}$. Disappearance of the SERS spectrum and the appearance of the carbon spectrum occur faster on glass. 107

Figure 5.19 SERS spectra of Rhodamine 6G $(1 \mu \mathrm{M})$ deposited on a monolayer of $\sim 60 \mathrm{~nm}$ silver nanocubes/DOPC on a glass (A,C,E, blue surfaces) and 7nm silicon film (B,D,F, red surfaces). Time progression of the spectra over 60 seconds is presented. Excitation wavelength of 458(A,B) or 488 (C-F) nm was used. Laser power was 25mW (A,B); $5 \mathrm{~mW}$ (C,D), and 50mW(E,F)... 108

Figure 6.1 Experimental scheme: silver nanocubes of 40 and 80nm average edge length were deposited by the Langmuir-Blodgett method onto a glass substrate containing a thin titanium oxide film of 0, 5, and 20nm thickness. Reflection (R) and transmission (T) spectra were measured for s- and p-polarization for front (left) or rear (right) illumination geometry. Absorption was calculated as $100-T-R$. Interparticle separation was controlled to minimize coupling. 118

Figure 6.2 AFM topography and TEM images of 80nm (A) and 40nm (B) cubes deposited on a dielectric substrate demonstrate nanocube size and interparticle separation. Nanocube density is indicated. Extinction spectra of nanocubes in suspension are presented as well. 120 Figure 6.3 Cross sections of extinction (solid lines) and absorption in front (dotted lines) and rear (dashed lines) illumination geometry measured for 80nm (top) and 40nm (bottom) silver nanocubes deposited on glass (left) and titanium oxide (5 and 20nm) substrates. Gray shaded areas indicate physical cross section. Quadrupolar and dipolar resonances are labeled

Figure 6.4 P-polarized light angle dependent reflection (R), transmission (T), and absorption (A) for a $40 \mathrm{~nm}$ nanocube sample on a $5 \mathrm{~nm}$ titanium oxide film. The signal is measured in \% 
for both rear (top) and front (bottom) illumination geometries.

Figure 6.5 S-polarized light angle dependent reflection (R), transmission (T), and absorption (A) for a $40 \mathrm{~nm}$ nanocube sample on a $5 \mathrm{~nm}$ titanium oxide film. The signal is measured in \% for both rear (top) and front (bottom) illumination geometries. 125

Figure 6.6 P-polarized light angle dependent reflection (R), transmission (T), and absorption (A) for an $80 \mathrm{~nm}$ nanocube sample on a 5nm titanium oxide film. The signal is measured in $\%$ for both rear (top) and front (bottom) illumination geometries. 125

Figure 6.7 S-polarized light angle dependent reflection (R), transmission (T), and absorption (A) for an $80 \mathrm{~nm}$ nanocube sample on a $5 \mathrm{~nm}$ titanium oxide film. The signal is measured in \% for both rear (top) and front (bottom) illumination geometries. 126

Figure 7.1 (A) UV-visible extinction spectra of a nanocube sample in ethanol (top) and on a polystyrene substrate in air (bottom). E-field distributions for the dipolar (D), quadrupolar (Q), and multipolar (M) resonances are shown for both environments. (B) TEM image of as synthesized cubes. (C) Histogram showing size distribution in terms of edge length for the sample. 139

Figure 7.2 (Left) Contour plot depicting the attenuation of light as a function of time. Contributions from plasmon modes are labelled as dipolar (D),quadrupolar (Q), and multipolar (M). (Right) Single extinction spectra corresponding to the horizontal lines on the contour plot. The red, green, and blue bands indicate the overall shift for D, Q, and M modes respectively. 140

Figure 7.3 Topographical evolution of a nanocube monolayer visualized by AFM. Average exposed cube height and duration of heating are indicated for each panel. .141 Figure 7.4 AFM images $\left(5 \times 5 \mu \mathrm{m}^{2}\right.$, left, and $1 \times 1 \mu \mathrm{m}^{2}$, right) of nanocube monolayers (A) initially and (B) after heating for $30 \mathrm{~min}$ at $112{ }^{\circ} \mathrm{C}$ 142 
Figure 7.5 Evolution of plasmonic modes. Relative change in wavelength of the dipolar resonance (red circles, relative change in intensities of the multimode resonances (blue triangles), and relative change in the wavelength of quadrupolar resonance (green square) versus time 145

Figure 7.6 Evolution of plasmonic modes as a function of nanocrystal depth. Red and green data points correspond to the normalized peak shift versus particle depth for the dipolar (D) and quadrupolar (Q) modes respectively. The blue data points represent the relative change in intensities of the multimode (M) resonances versus depth of nanocube immersion. Fitted lines are empirical and based on a mono-exponential decay for $\mathrm{D}$, and logistic functions for $\mathrm{Q}$ and $\mathrm{M}$ modes. 146

Figure 7.7 FDTD calculated extinction spectra of a silver nanocube in the polystyrene film for different embedding depths. E-Field distributions (imbedded) are given for the initial and final states of the simulation. The red, green, and blue bands indicate the overall shift for $\mathrm{D}$, $\mathrm{Q}$, and $\mathrm{M}$ modes respectively..... 149

Figure 7.8 Peak shifts from FDTD modelling displaying: (A) normalized peakshift versus depth of immersion for a normal oriented $62 \mathrm{~nm}$ cube imbedding into polystyrene. (B) Relative peak shift against angle of tilt about a single axis for a $62 \mathrm{~nm}$ cube held at a constant depth of $40 \mathrm{~nm}$ in polystyrene. Very little change is observed at small angles $\left(<15^{\circ}\right)$, but increasing the angle of tilt results in a small blue shift of the D mode and a rapid red shift of the Q mode. .151

Figure 7.9 Experimental schematic: A monolayer of silver nanocubes deposited via the Langmuir-Schaefer technique onto a thin $50 \mathrm{~nm}$ film of polystyrene on a $20 \mathrm{~nm}$ thin film of high refractive index titanium oxide. As the system is heated near the glass transition temperature of the underlying polymer the nanocubes begin to diffuse into the polymer layer. 
The position of the nanocubes and corresponding extinction spectrum are shown for the initial (A), intermediate (B), and final (C) position. Due to the high refractive index of the underlying titanium oxide, the D mode shifts further when compared to polystyrene on glass system. The Q mode only shifts marginally as the cubes themselves are larger than the polymer layer and a large portion of the cube remains exposed keeping the Q mode sensitive to the surrounding environment (air) above the composite material...............................154 


\section{Abbreviations}

AFM - Atomic Force Microscopy

AgNC - Silver Nanocube

CCD - Charge-Coupled Device

DPPE-RhB - 1,2-Dipalmitoyl-sn-glycero-3-phosphoethanolamine- $N$-(lissamine rhodamine B sulfonyl)

EELS - Electron Energy Loss Spectroscopy

EG - Ethylene Glycol

EM - Electromagnetic

FFT - Fast Fourier Transform

FOM - Figure of Merit

FRET - Förster Resonance Energy Transfer

FWHM - Full Width at Half Maximum

GA - Glycolaldehyde

IR - Infrared

LB - Langmuir Blodgett

LSPR - Localized Surface Plasmon Resonance

NSET - Nanometal Surface Energy Transfer

PTFE - Polytetrafluoroethylene

PS - Polystyrene

PVP - Polyvinylpyrrolidone

RI - Refractive Index

RIS - Refractive Index Sensitivity

RIU - Refractive Index Unit

SB - Scheafer Blodgett

SEM - Scanning Electron Microscopy

SERS - Surface Enhanced Raman Spectroscopy

SPP - Surface Plasmon Polariton

SPR - Surface Plasmon Resonance

TEM - Transmission Electron Microscopy

UV-Vis - Ultraviolet Visible 
Chapter 1

Introduction 
"Light has a relation to the matter which it meets within in its course, and is affected by it, being reflected, deflected, transmitted, refracted, absorbed, \&c. by particles very minute in their dimensions"

-Micheal Faraday, 1857

\subsection{Historical Development}

Coinage metal nanoparticles have been in use for more than a millennia, beginning with the production of stained glass materials. The earliest known use is the Lycurgus cup (Figure 1.1), a $4^{\text {th }}$ century artifact from the Roman Empire. ${ }^{1}$ The cup contains gold nanoparticles giving it a dichroic property in which the transmission of light is red, while its reflection is green.

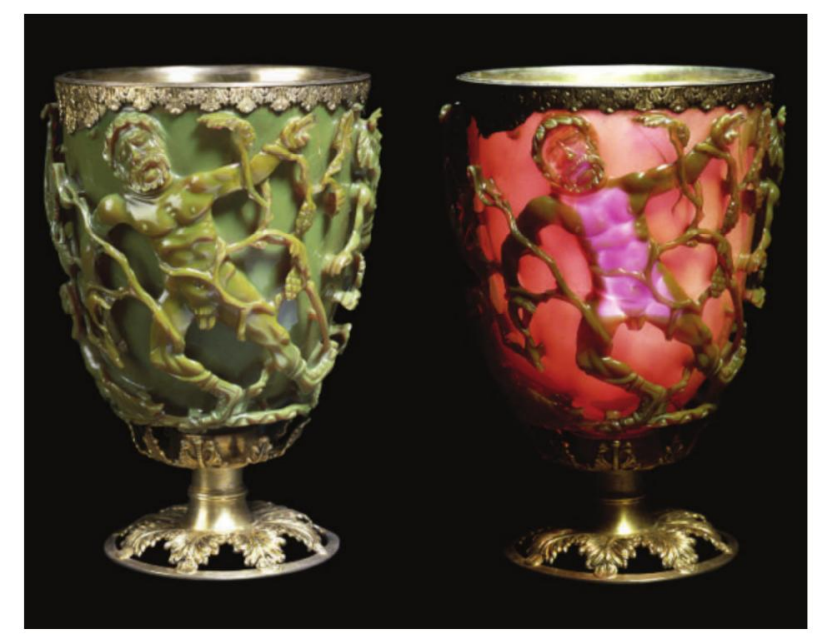

Figure 1.1: Lycurgus Cup, (British Museum, Fourth Century AD), This Roman cup contains gold nanoparticles making it dichroic. Reflected light gives it a green colour, but it attains a ruby red when viewed with transmitted light. Reproduced with Permission. ${ }^{1}$ Copyright 2007 Nature Publishing Group.

Alchemists and iatrochemists such as Paracelcus in the $16^{\text {th }}$ century, were producing solutions of stable gold nanoparticles for use as a cure-all due to the resemblance of the fluid to blood. The unparalleled beauty of these stained glass materials continued to captivate audiences, particularly artists and glass makers. The first known treatise on glass making 
L'Arte Vetraria published in 1612 by Antonio Neri, a Florentine priest and glass maker who provided a description on how to produce ruby red glass by the application of gold in the process. ${ }^{2}$ While the production of theses vibrant glasses and fluids had been well established centuries ago, there was no true insight into the actual origin of the phenomena as there was no way to know that the optical properties were a consequence of nanoscale particles. Although the idea of very small gold particles being responsible for the resulting colours was suggested by German chemists Johann Kunckel and Jeremais Benjamin Richter, it was not until a detailed study by the English scientist Micheal Faraday published in 1857 that produced a wide array of experimental evidence to support this hypothesis. ${ }^{3}$ Half a century later, using the electromagnetic theory of light developed by Scottish scientist James Clerk Maxwell, German physicist Gustav Mie derived exact solutions to Maxwell's equations for the scattering of light by spherical particles building a theoretical foundation for the observed phenomena of gold colloids. ${ }^{4,5}$

Up to the 1950s, this general phenomena was simply referred to to as 'Mie scattering', as the idea of a plasmon had not yet been realized. Pioneering work done by David Bohm and David Pines studying the collective oscillations of electrons in metals, led Pines to coin the term 'plasmon' short for plasmon oscillations, deriving the name from their analogous similarities to plasma oscillations previously discovered in the gas phase by Irving Langmuir. $^{6-8}$ This type of electron oscillation is referred to as a bulk or volume plasmon as it occurs within the body metal, and primarily measured by electron energy loss spectroscopy (EELS); the main tool used to investigate bulk plasmons and develop the theory behind them. Shortly after this development Rufus Ritchie demonstrated that metals could support an additional type of plasmon confined to the surface of the metal. ${ }^{9}$ These types of 
oscillations were aptly named surface plasmons, which at the time were still primarily investigated through the application of EELS. It wasn't long before the discovery that surface plasmons could be probed optically under specific conditions such as the use of a high refractive index prism, or by production of a diffraction grating directly on the metal

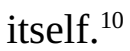

The application of surface plasmons as a tool and the first experimental insight into their near-field behavior began accidentally with the discovery of Surface Enhanced Raman Spectroscopy (SERS), which was first observed by Martin Fleischmann in 1973 while observing an unusually intense Raman spectrum of pyridine on a roughened silver film. ${ }^{11}$ It was soon confirmed that this anomalous increase in intensity of the Raman signal could not be accounted for by concentration even when the dramatic increase in surface area of the film was taken into account, so in 1977 Richard van Duyne proposed that an electric field enhancement in the vicinity where the Raman labels were located was responsible. ${ }^{12}$ In the same year Alan Creighton explained the phenomenon via a charge-transfer effect resulting from the direct interaction of the molecule with a surface plasmon. ${ }^{13}$

Near the end of the $20^{\text {th }}$ century, a rapid progression of synthetic methods for metal nanoparticles and lithographic fabrication techniques with unprecedented control and resolution on the nanoscale occurred. Coupled with the wide spread adoption of electron microscopy, it suddenly became possible to rationally and purposefully engineer metal nanomaterials that could support surface plasmons for study and application specifically, creating the interdisciplinary field now known as plasmonics. The growth of this field is highlighted by Figure 1.2 showing the approximate number of publications in scientific literature that contain the term 'Surface Plasmon' from 1965 to 2015. 


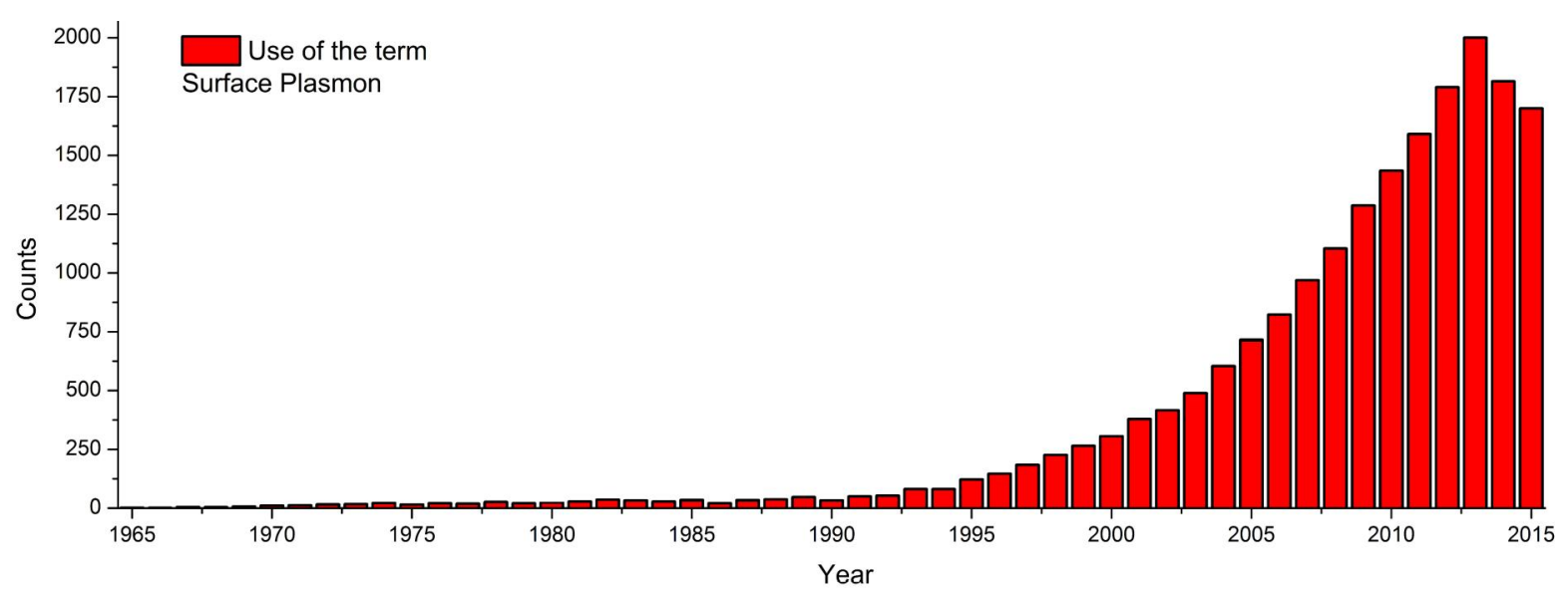

Figure 1.2: Appearance of the term 'Surface Plasmon' in scientific literature. SciFinder Scholar@

\subsection{Fundamentals of Plasmonics}

From a quantum mechanical view, plasmons can be considered as collective excitations of electrons in the conduction band of a metal oscillating around the positive charge provided by the nuclei of the metal itself. The movement of the electrons generates electron-hole pairs resulting in Coulombic attraction acting as a restoring force against the driving force of an incident electromagnetic (EM) wave.

Classically, a plasmon can be described as a local polarization in a metal induced by an external driving force (incident radiation). All classical approaches to modeling a plasmon require solving Maxwell's equations and are fairly accurate provided a good description of the dielectric function of the metal is used, and appropriate boundary conditions are established. The application of Maxwell's equations is sufficient to accurately describe the optical properties of plasmonic systems down to just a few nanometers in diameter.

Inside the body of a metal, plasmon oscillations are referred to as bulk or volume plasmons. As mentioned earlier, one of the parameters required for a classical model of plasmons is an accurate description of the dielectric function. The simplest model for 
estimating the dielectric function of a metal is the Drude model which treats electrons as a gas that is non-interacting with itself and only able to relax though collisions with the metal lattice. Using this model it is possible to attain a bulk plasma frequency $\omega_{\mathrm{p}}$ described by equation $(1.1) .14,15$

$$
\omega_{p}=\sqrt{\left(\frac{N e^{2}}{m \epsilon_{0}}\right)}
$$

Where $\mathrm{N}$ is the concentration of free electrons in the metal, e and $\mathrm{m}$ are the charge and mass of an electron respectively, and $\varepsilon_{0}$ is the permittivity of free space. Bulk plasmons propagate as longitudinal waves, so they do not couple directly to light as EM radiation is a transverse wave. The work of Rufus Ritchie on the nature of plasmons occurring at the surface of a metal lead to the discovery that these oscillations can also propagate along the surface and can be driven by optical excitation with the use of a high refractive index (RI) prism. The coupling between a surface plasmon and incident radiation has the formal name of Surface Plasmon Polariton (SPP). ${ }^{16}$

If one continues to confine the dimensions of the system from a two dimensional sheet to a one dimensional wire, guided plasmon modes that are are strongly optically active begin to emerge. Using the bulk plasma frequency from the Drude model (1.1) it becomes possible to derive the surface plasmon resonance (SPR), equation (1.2). ${ }^{14,17}$

$$
\epsilon_{r}=1-\frac{\omega_{p}^{2}}{\omega^{2}+\gamma^{2}}
$$

Where $\varepsilon_{\mathrm{r}}$ is the real component of the dielectric function for a metal, $\omega$ is the surface 
plasmon frequency, $\omega_{\mathrm{p}}$ is the bulk plasmon frequency, and $\gamma$ is the dampening frequency given by the bulk metal which is based on the mean free path of the conducting electrons $R_{\text {bulk }}$ and the velocity of the electrons at the Fermi energy $v_{\mathrm{f}}$ in equation (1.3).

$$
\gamma=v_{f} / R_{\text {bulk }}
$$

When considering an SPR that occurs in the ultraviolet (UV) and visible regions $\gamma<<\omega_{\mathrm{p}}$ and equation (1.2) can be simplified:

$$
\epsilon_{r}=1-\frac{\omega_{p}^{2}}{\omega^{2}}
$$

Solving for the surface plasmon frequency yields:

$$
\omega=\frac{\omega_{p}}{\sqrt{\left(2 \epsilon_{r}+1\right)}}
$$

Further confinement to a zero dimension material (all three dimensions are well below the diffraction limit) plasmons can no longer propagate and are referred to as Localized Surface Plasmons. If we reconsider the damping frequency given by equation (1.3), and compare the mean free path of the conduction electrons $R_{\text {bulk, }}$ and the physical radius of the particle $R$, confinement effects begin to occur. This yields an effective radius $R_{\text {eff, }}$ when the particle radius is smaller than the natural mean free path of the electrons causing them to scatter off the surface of their now limited domain. This relationship is given by equation (1.6), ${ }^{14}$ and causes strong size dependencies on the damping frequency and subsequently the resonance condition.

$$
1 / R_{\text {eff }}=1 / R+1 / R_{\text {bulk }}
$$


In order to set a resonance condition, Gustave Mie's solutions to Maxwell's equations to describe the scattering of EM radiation by a sphere must be considered. Equation (1.7) describes the extinction cross section $C_{\text {ext }}$ of a small metal sphere: ${ }^{14}$

$$
C_{e x t}=\frac{24 \pi^{2} R^{3} \epsilon^{3 / 2}}{\lambda}\left[\frac{\epsilon_{i}}{\left(\epsilon_{r}+2 \epsilon_{m}\right)^{2}+\epsilon_{i}^{2}}\right]
$$

Where $\mathrm{R}$ is the radius of the particle, $\varepsilon_{\mathrm{m}}$ is the real dielectric function of the surrounding material, $\lambda$ is the wavelength of the incident EM radiation, $\varepsilon$ is the dielectric function for the metal, and $\varepsilon_{\mathrm{r}}$ and $\varepsilon_{\mathrm{i}}$ are the real and complex components of the dielectric function for the metal respectively. This equation allows for a resonance condition to be set when $\varepsilon_{\mathrm{r}}=-2 \varepsilon_{\mathrm{m}}$, provided $\varepsilon_{\mathrm{i}}$ is sufficiently small. This condition allows equation (1.5) to be rewritten in more convenient terms of wavelength of incident radiation $\left(\lambda_{\max }\right)$ and effective refractive index of the surrounding medium $\left(\mathrm{n}_{\mathrm{m}}\right)$ :

$$
\lambda_{\max }=\lambda_{p} \sqrt{\left(2 \epsilon_{m}+1\right)} \simeq \sqrt{2} \lambda_{p} n_{m}
$$

Setting this condition results in a maximum extinction cross section $C_{\mathrm{ext}}$ at a frequency were the optical extinction will be at its highest, but also shows that the position of this maximum extinction is dependent on the refractive index of the surrounding environment of the particle.

When a particle is irradiated on resonance, the resulting plasmon organizes itself into pairs of alternating positive and negative regions called modes. The simplest mode is a dipolar resonance, characterized by a pair of oscillating regions of electron density (Figure 
1.3). The dipolar mode is the simplest, lowest energy resonance, and usually the most intense.

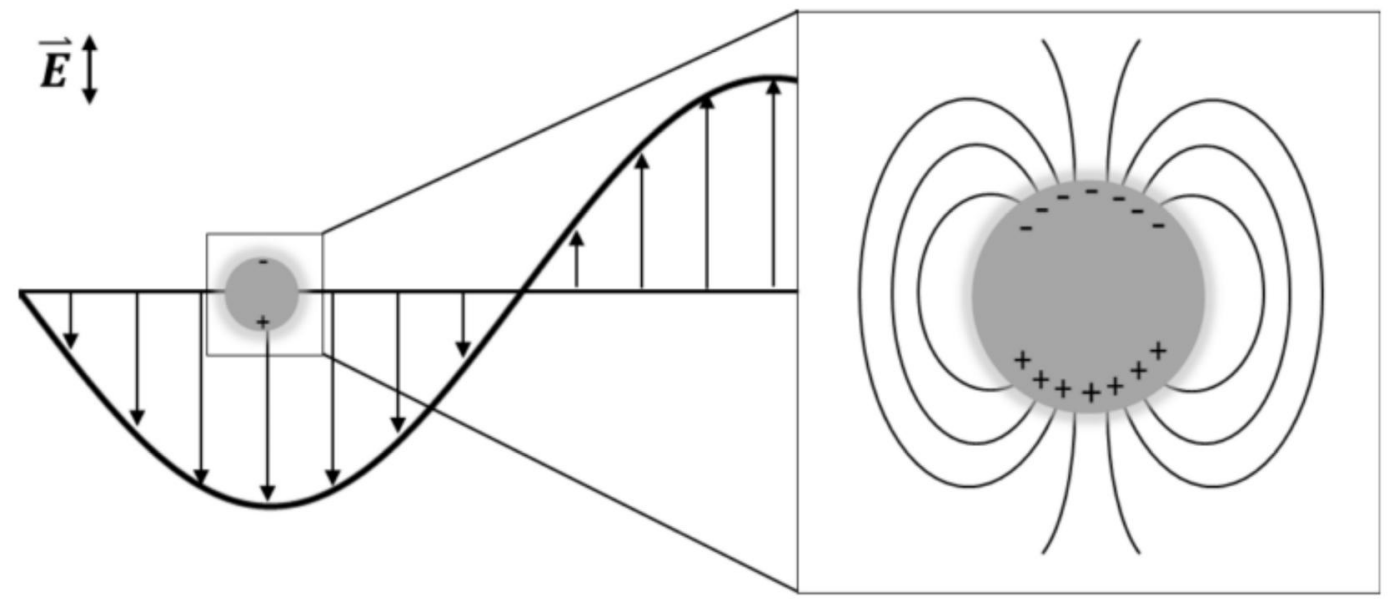

Figure 1.3: Schematic illustration of a dipolar localized surface plasmon. Conducting electrons are displaced by the electric component of resonant frequency EM radiation. This leads to a strong optical extinction as well as an enhanced electric field surrounding the particle itself. Reproduced with permission. ${ }^{85}$ Copyright 2014 Daniel Prezgot, Carleton University.

Particles can have several modes of various order occurring at different energies. Each mode can produce a peak in the extinction spectrum of the particle and has its own refractive index sensitivity (RIS) that can be described by its figure of merit (FOM), given by equation (1.9). This equation takes the magnitude of the spectral shift as well as the peak width into account:

$$
F O M=\frac{\Delta \lambda}{\lambda_{F W H M}}
$$

Where $\Delta \lambda$ is the peak shift in nanometers per refractive index unit (nm/RIU), and $\lambda_{\text {FWHM }}$ represents the full width of that peak at half intensity in nanometers. This simply means an observed shift is more significant if it is both highly sensitive and very narrow.

The requirements for a material to support a surface plasmon require both a large negative real dielectric function, and small complex dielectric function. The relationship 
between these two parameters also determines the strength or quality of a plasmon. The quality factor for a particle can be approximated by equation (1.10) which is simply the negative ratio of the two components. ${ }^{18}$

$$
Q=-\frac{\epsilon_{r}}{\epsilon_{i}}
$$

It is important to note that equation (1.10) does not take inter-band transitions within the metal into account so additional losses will occur at energies where such transitions take place, and as the dielectric functions of the metal are a function of wavelength, so too is the quality factor.

\subsection{Metals that Support Plasmons}

As stated in the section above, the resonance condition is set by $\varepsilon_{\mathrm{r}}=-2 \varepsilon_{\mathrm{m}}$, when $\varepsilon_{\mathrm{i}}$ is sufficiently small. This means for a metal to be able to support an LSPR or SPP the real part of the dielectric function must be large and negative, while the complex part must be small at the specified wavelength for any particular metal. This relationship is exemplified in Figure 1.4, showing the real and complex values of $\varepsilon$ for silver, gold, and silicon in the visible region. ${ }^{19}$
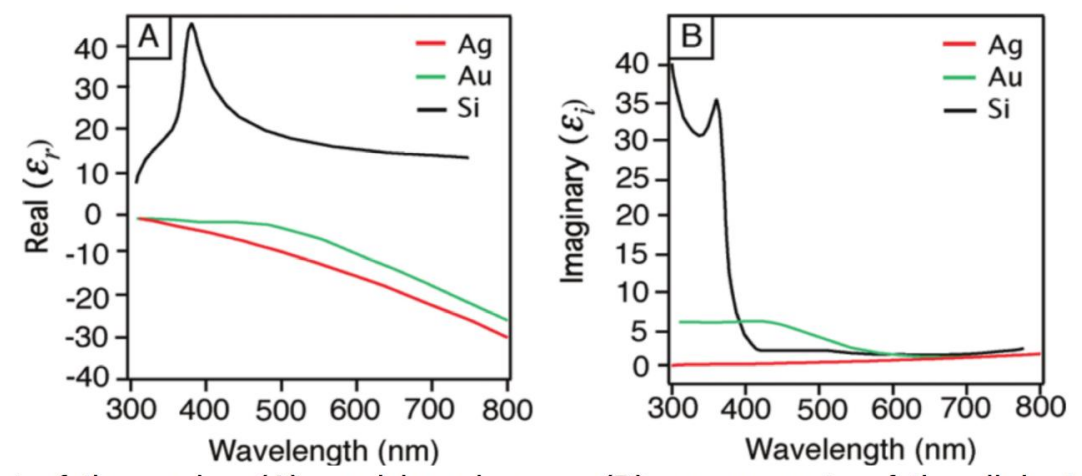

Figure 1.4: Plot of the real $\varepsilon_{\mathrm{r}}(\mathrm{A})$, and imaginary $\varepsilon_{\mathrm{i}}(\mathrm{B})$ components of the dielectric functions for silver, gold, and silicon in the visible spectrum. Reproduced with permission. ${ }^{19}$ Copyright 2011 American Chemical Society. 
Gold and silver are the most commonly used and studied metals for plasmonic applications to date as they are easily synthesized and numerous complex shapes are currently known. ${ }^{20,21,19}$ Gold is also a bio-compatible material, making it a strong candidate for biological studies and applications. While silver is not as chemically stable as gold, it has the highest quality factor of plasmonic materials making it outperform other materials in terms of interaction strength particularly in the visible region. ${ }^{18}$ Despite their ubiquitous use, plasmonics are not limited to the coinage metals. Much of the periodic table can support surface plasmons (Figure 1.5).

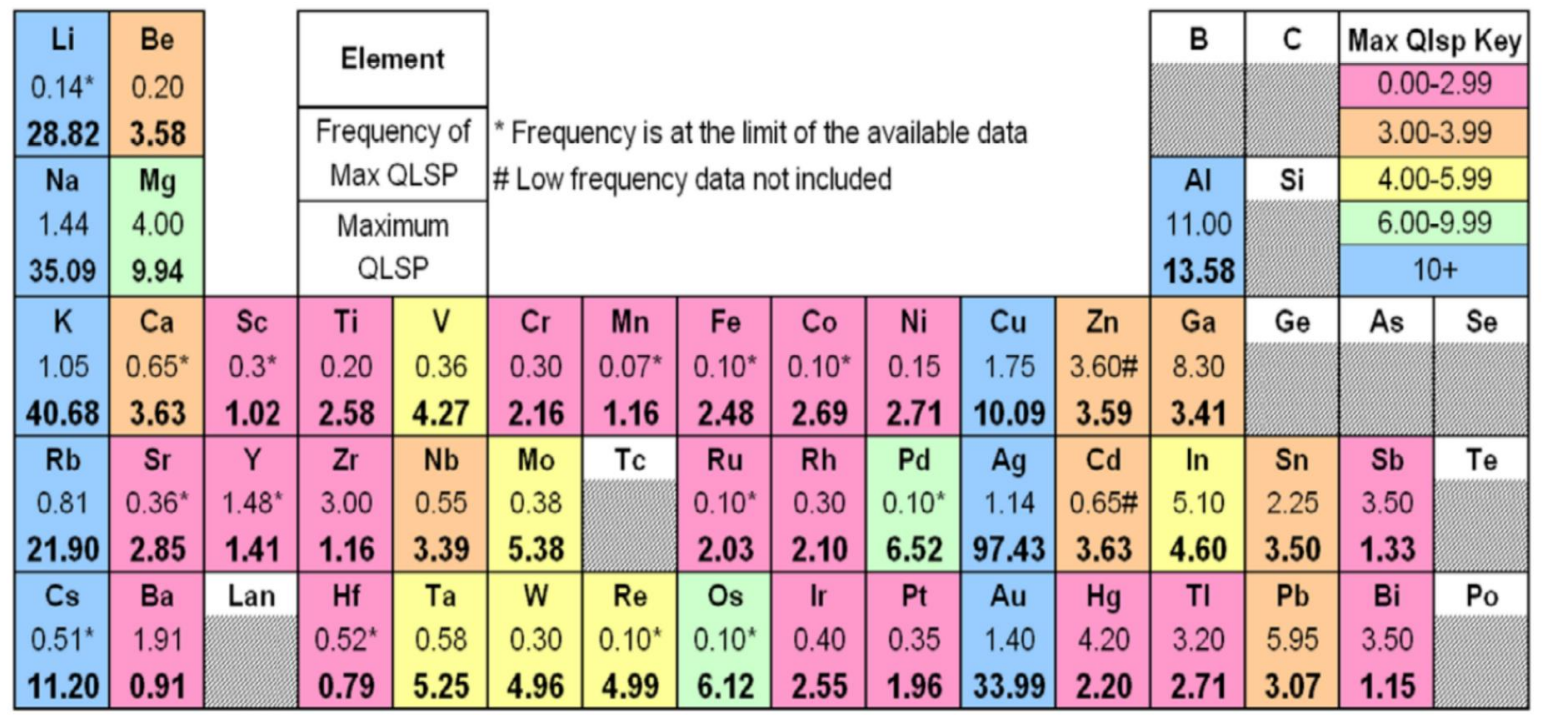

Figure 1.5: Periodic table of the elements showing the highest quality factor attainable for each element. Frequencies are in eV. Reproduced with Permission. ${ }^{18}$ Copyright 2010 IOP Publishing.

The alkali metals for example, all support high quality plasmons $(>10)$ and do not experience the losses from inter-band transitions associated with silver and gold in the UV region, ${ }^{18}$ their highly reactive nature however makes them difficult to use in their metallic form. Similarly aluminum has gained increased attention and interest as a plasmonic material as it can support an LSPR in the UV region of the spectrum, that is passivated by a hin layer of aluminum oxide. ${ }^{22}$ In addition to elements, there is a fairly extensive list of 
compounds that can support plasmons including various metal alloys, semiconductors, conductive oxides, nitrides, and silicides. ${ }^{23}$ Many of these are considered 'alternate plasmonic materials' to gold and silver, some with attractive physical properties such as refractory plasmonics which are materials that can support a surface plasmon but also have high chemical durability and high melting points $\left(>2000^{\circ} \mathrm{C}\right)$. This includes materials such as ZrN, TiN, Ta, Mo, and W. ${ }^{24}$

\subsection{Effect of Local Environment}

Plasmonics and their properties are very sensitive to numerous factors including size, shape, material, and local environment such as proximity to other particles, metal films, and dielectrics. Numerous configurations are possible has allowed for significant control over near-field and far-field properties in various plasmonic systems for numerous applications, while also yielding insight into the fundamental nature of plasmons and their behavior.

\subsubsection{Size and Shape}

Gustav Mie used Maxwell's equations to determine the exact solution for a sphere of arbitrary size. Only a small number of exact solutions are known, and only for simple geometries such as spheroids, and cylinders. ${ }^{25}$ For particles of lowered symmetry approximate solutions can only be found by the use of numerical methods and comparing them to experimental results. Since EM radiation is a transverse wave, plasmons will be excited in the direction corresponding to the oscillation of the electric field component. As described in equation (1.6), the frequency of a plasmon is strongly size dependent. Modes appear red shifted as the particle increases in size, and higher order modes will begin to emerge. If one compares a spherical particle to an oblong particle that is continuously elongated along a single axis, what began as a degenerate set of dipole resonances in the 
sphere becomes two separate dipoles each associated with their propagation length(s). This dependence becomes very clear when polarized light is used on a system with very high anisotropy, such as the localized and propagating modes present in a nanowire. ${ }^{26,27}$ These discreet modes can be accessed independently by simply aligning the wires with the correct polarization and wavelength of incident light. When considering a particle that has sharp corners and edges such as stars, cubes, octahedrons, and other polyhedrons, a redshift in the dipolar mode is observed when compared to a spherical particle with the same diameter. As the symmetry is further lowered, the emergence of higher order modes can occur, always at energies greater than the initial dipolar mode. ${ }^{25}$ The existence of multipolar modes in a nanoparticle is dependent on size, shape, asymmetry in the local environment, and the metal itself. While most small particles only have a single dipolar mode observable in their extinction spectrum, silver nanocubes (AgNC) for example, begin supporting a higher order mode at very small edge lengths of $18 \mathrm{~nm} .{ }^{28}$ Larger AgNCs with $80 \mathrm{~nm}$ edge lengths have four modes visible in their extinction spectrum, compared to only a single dipolar mode observed for similarly sized gold nanocubes. ${ }^{21}$

\subsubsection{Inter-particle Coupling}

One of the more dramatic effects observed by plasmonic materials occurs when they are in close proximity to another metal such as a particle or film. Interactions between adjacent systems results in significant changes in the extinction spectrum including a decrease in frequency of the observed modes of isolated particles, and the generation of a new mode at an even lower energy. This interaction is referred to as coupling, and one of the most common examples of this is the use of gold nanoparticles that aggregate when a specific target molecule is introduced into the system, causing a visible change in the sample 
from red to blue. In the simplest model for this effect, the charge oscillation in one particle induces the same oscillation in a neighboring particle resulting in a coupling band that appears at a frequency lower than the inherent dipoles of the isolated particles. This also leads to the generation of so called 'hot spots' where the electric field experiences significant enhancement locally within the gap between particles. This phenomenon can be directly probed by using SERS, fluorescence, or near-field scanning optical microscopy, and can have dramatic impact on the far field spectral properties of a system making it a powerful tool for sensing and spectral engineering.

\subsubsection{The Plasmon Hybridization Model}

Plasmonic interactions can become complicated when multiple variables are considered simultaneously. A method developed for visualizing and discussing the effects proximity has on a system, referred to as the plasmon hybridization model developed by the Nordlander group at Rice University in $2003^{29}$ is depicted in Figure 1.6.

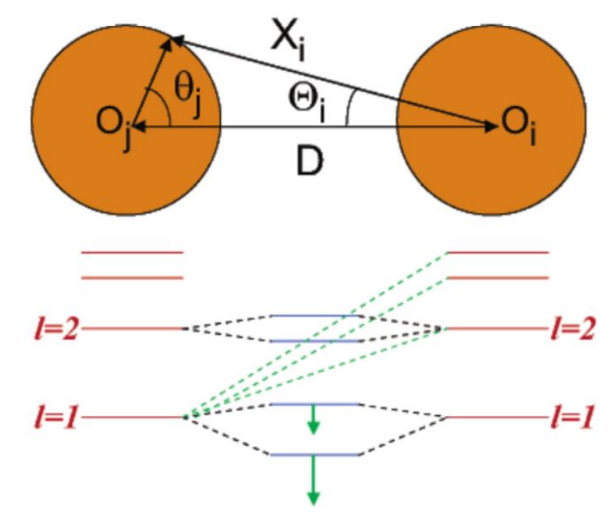

Figure 1.6: Schematic illustrating plasmon hybridization in a nanoparticle dimer. The plasmons in each particle interact with each other in both a constructively and deconstructively in a similar manner to bonding and anti-bonding orbitals in molecules. This interaction generates a bright coupling mode. Interactions between modes in adjacent particles lowers their energy causing red shifts in their peak positions. Reproduced with Permission. ${ }^{34}$ Copyright 2004 American Chemical Society

The model treats plasmon modes in the same manner as electron orbitals, allowing for 
constructive (bonding) and destructive (anti-bonding) plasmons to form between adjacent particles (or metal film). This interaction gives rise to the bright coupling mode observable in an extinction spectrum for particle dimers discussed in the last section, but also yields additional insight into the interaction by providing an explanation for the observed lowering in frequency for the inherent modes when coupling occurs, and the strong distance dependent behavior for coupling.

\subsubsection{Higher Order and Dark Modes}

When a plasmon mode is optically excited, the electrons oscillate in-phase with the incident electric component. These radiation driven interactions are what lead to the strong peaks observed in the extinction spectrum of plasmonic particles and are referred to as bright, or super-radiant modes. The anti-phase oscillation of plasmonic modes is still possible, but cannot be driven by incident radiation and thus are referred to as dark, or subradiant modes. These dark modes can be accessed and mapped using EELS, ${ }^{30}$ but they can also be accessed through coupling to bright modes within the particle or system. Coupling between bright and dark modes can be achieved by anisotropy in the particle, its local environment, or by coupling to anther particle or metal film. ${ }^{31-34}$ Dark modes do not radiate energy like bright modes, and are exclusively a near-field phenomenon. ${ }^{32}$ This lack of radiative decay in dark modes gives them a longer lifetime than bright modes, ${ }^{35}$ which can be utilized as an efficient way to route electromagnetic energy over relatively large distances (microns) confined in a structure well below the diffraction limit. ${ }^{36}$

Higher order modes (modes that have more than two discreet regions of oscillating electron density), have a much more narrow peak width than dipolar modes, and are more closely localized to the surface of the particle. This impact can be seen with SERS, as the 
spatial volume of the enhanced field is smaller, and in the refractive index FOM. Dipolar modes have the highest refractive index sensitivity in most systems, but due to the significantly smaller peak width associated with higher order modes, they can often outperform dipolar modes in terms of FOM. ${ }^{37}$

\subsubsection{Substrate Interactions}

Plasmonic particles interact strongly with thin metal films leading to the generation of highly distance dependent coupling bands with strong electric field intensity between the particle and the film. This effect is very similar to inter-particle coupling and can also be described by the plasmon hybridization model, resulting in similar red shifts in the inherent modes and a new lower energy bright mode. ${ }^{33}$ Plasmonic particles are also sensitive to the proximity of a dielectric substrate. While a coupling mode with the surface is not generated in this interaction, proximity to a dielectric does have a strong influence on the type, position and number of plasmon modes present in the particle. This occurs by particles interacting with the image of their surface charges in the dielectric itself depicted in Figure (1.7).
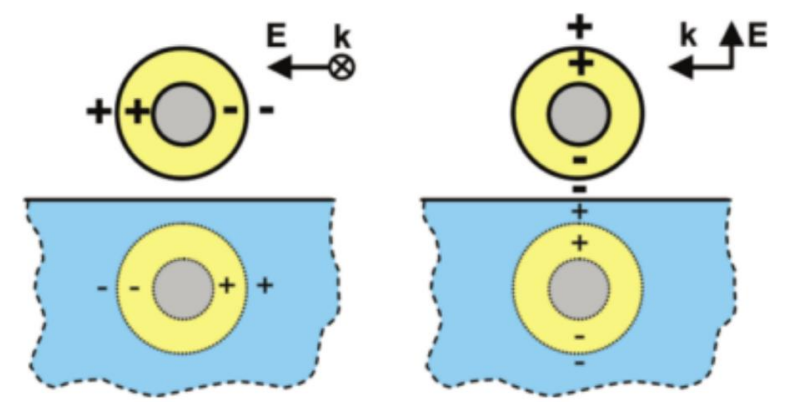

Figure 1.7: Schematic showing the polarization dependent substrate interactions between a plasmonic nanoparticle and a dielectric substrate. Reproduced with Permission. ${ }^{38}$ Copyright 2009 American Chemical Society

Similarly to the generation of coupling bands, the plasmon hybridization model accurately describes the formation of hybrid modes. The asymmetry in the local environment surrounding the particle is what gives rise to hybrid modes, that occur at different 
frequencies with their own polarization dependencies. ${ }^{38-40}$ This asymmetry distributes the plasmons differently across the particle, and as this effect is based on proximity to a dielectric material, therefore it is not surprising that contact area (or near contact area) plays a significant role in the strength of surface interactions. For example, if one selects a planar substrates and a nanoparticle with flat faces such as a cube, the interaction becomes stronger than with tangential contact, leading to the generation of two highly spatially and energtically resolved plasmon modes. ${ }^{41,42}$ This interaction can be thought of as a hybridization of the inherent dipolar mode into two new hybrid modes localized at opposite ends of the particle itself, Figure (1.8).

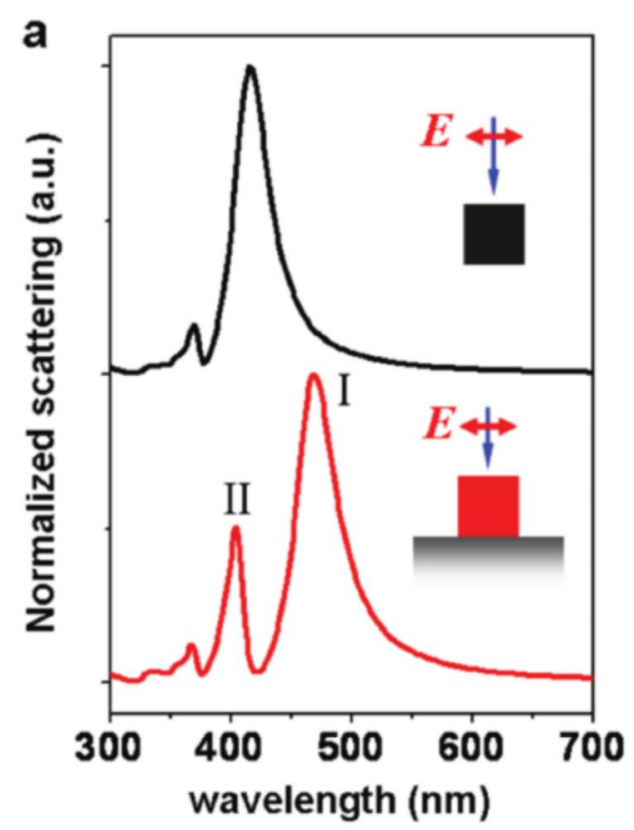

b

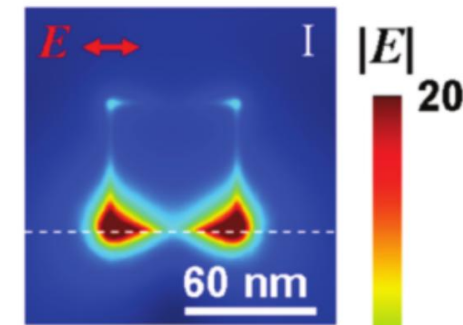

C

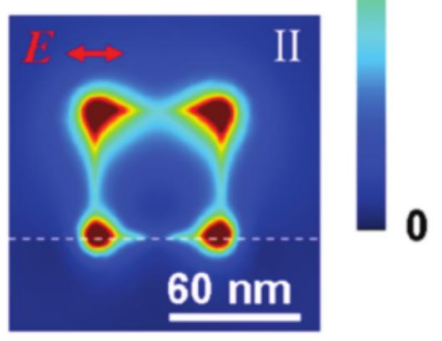

Figure 1.8: (a) Simulated scattering spectra from a cube in vacuum (black line) and a cube on a glass substrate (red line). (b) and (c) correspond to the electric field amplitude for peaks I and II from the red curve respectively. Reproduced with Permission. ${ }^{42}$ Copyright 2011 American Chemical Society

As these new hybrid modes are spatially separated, each mode responds to changes in its specific local environment allowing for independent tuning of the spectral positions for both modes by simply changing the refractive index of the substrate and the surrounding medium. 
The high strength of this interaction for silver nanocubes is due partially to the ability of silver cubes to hold higher order modes at small particle sizes, combined with the high quality factor of silver metal.

\subsubsection{Fano Interferences}

In 1961, physicist Ugo Fano published a paper describing asymmetric line shapes observed in spectra for electrons scattering off of helium atoms. He attributed the spectral feature to a configuration interaction where the energy states of a continuous (broad) state coincides with the energy of a discreet (narrow) state. ${ }^{43}$ This interaction is general and not limited to electron scattering spectra. Fano resonances have been observed arcross disciplines including nuclear physics, circuitry, photonics and optics, and plasmonic systems. $^{44}$

Fano interference patterns can be generated in plasmonic nanostructures via coupling to the inter-band transitions within a metal present in the system, or by coupling to dark modes that are either inherent in the system or induced by interactions such as particle coupling or substrate effects that introduce anisotropy in the local environment. A good example of a Fano interference that arises from interaction of an inter-band transition and a LSPR can be seen in heterogeneous dimers of gold and silver nanoparticles. The relatively higher energy plasmon modes of the silver particle overlap with the inter-band energy within the gold metal leading to a polarization dependent loss that has the characteristic asymmetric Fano line shape observable in the extinction spectrum. ${ }^{45,46}$

Fano interferences in plasmonic systems can still occur in the absence of an interband transition resulting from the hybridization interactions between bright modes in strongly coupled systems. The requirements for this type of interference to occur can be 
accurately described by the plasmon hybridization model for a highly coupled plasmonic system with several components. ${ }^{47}$ By altering the distances between components within a system, significant spectral shifts occur through the generation of hybrid bright and dark modes. It then becomes possible to tune the spectral position of the bright and dark modes generating an interference band (Figure 1.9) which can be either constructive or deconstructive in nature.

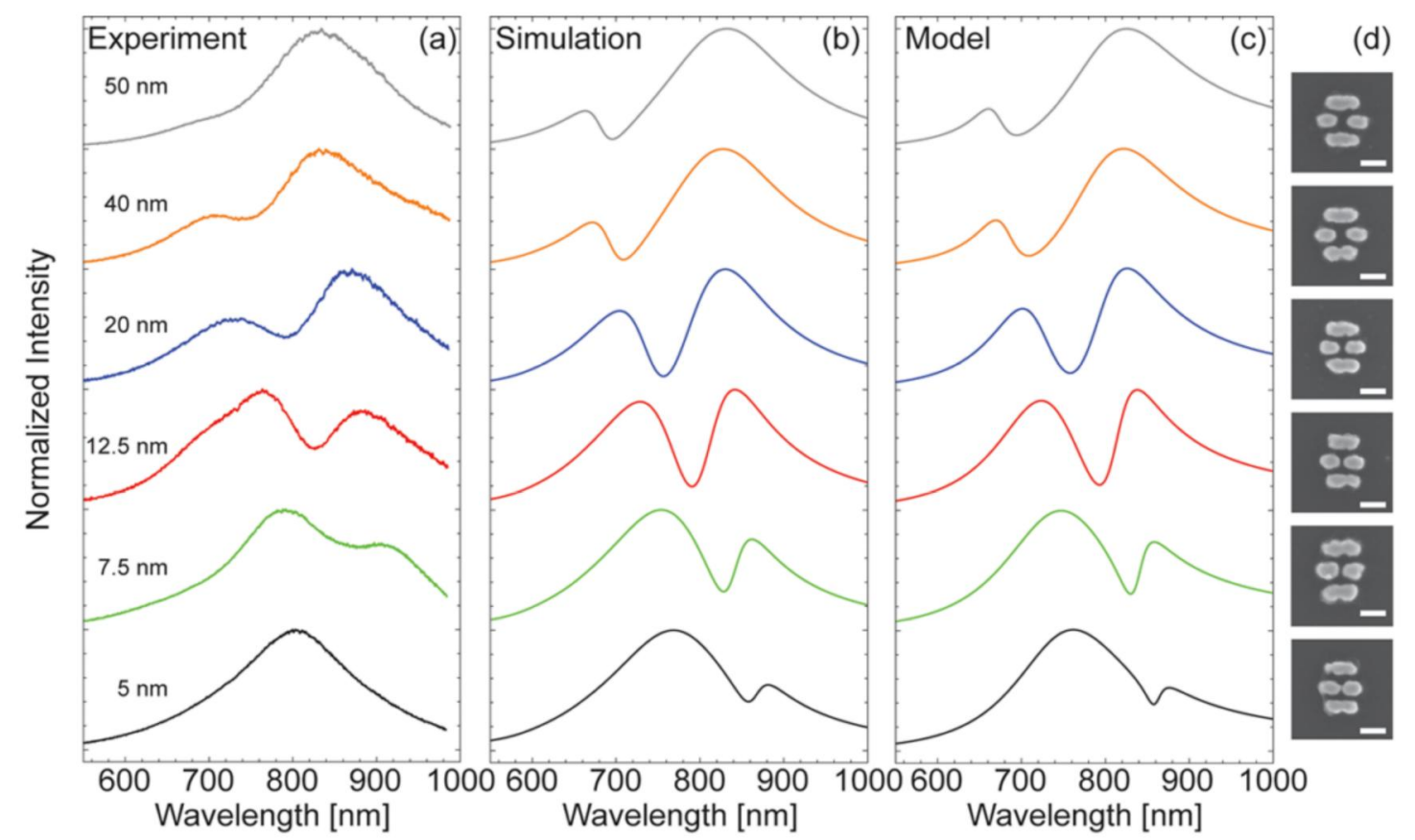

Figure 1.9: Fano interferences caused by hybridization of plasmon modes in an array of 4 gold nanobars as the distance between the two middle bars is varied. (a) Experimental dark-field scattering spectra. (b) FDTD simulated scattering spectra. (c) Plasmon hybridization model calculated spectra. (d) SEM images of the measured arrays. Reproduced with Permission. ${ }^{47}$ Copyright 2013 American Chemical Society.

\subsection{Applications}

This section outlines some of the current applications in plasmonics with resonances occurring mostly in the ultraviolet-visible region of the spectrum. There is a great deal of research and application related to photonic/plasmonic devices designed to operate at infrared frequencies that is not discussed here. ${ }^{48,49}$ 


\subsubsection{Sensing}

Plasmonic materials are highly sensitive to the refractive index of their local environment, making them an ideal candidate for direct refractive index sensing. As discussed above, different materials, shapes, sizes, and local environments all contribute to the nature of the plasmonic mode(s) that occur and their spectral location(s). This allows for the rational design for highly sensitive refractive index sensing anywhere from the

ultraviolet to the near-infrared. ${ }^{42,50-53}$ While refractive index sensing itself is non-specific, the sensitivity of the particles is to that of their local environment, as such if one makes the local environment selective, this in turn makes the plasmonic sensor also selective. One classic configuration of this is the use of a SPR sensor first devised by E. Kretschmann and A. Otto in 1968, depicted in Figure 1.10. ${ }^{10}$

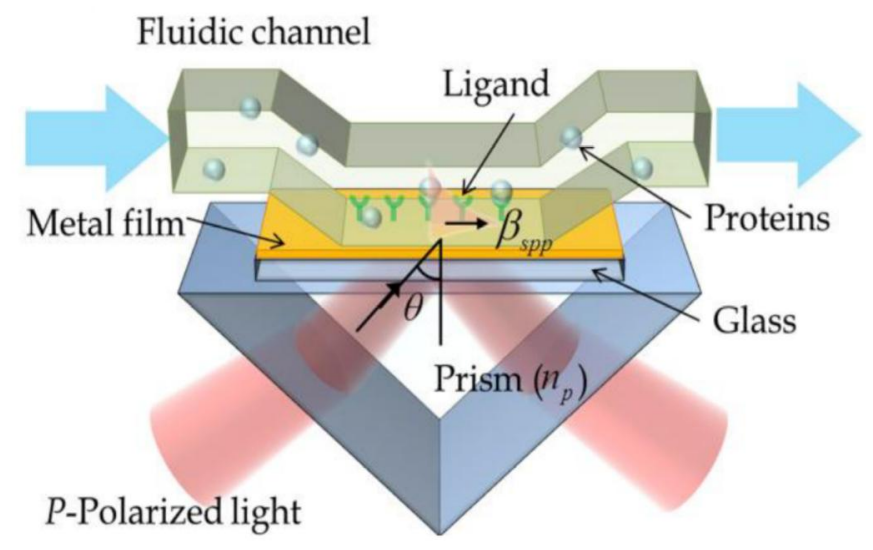

Figure 1.10: Example of a SPR sensor in the Kretschmann configuration. The resonant frequency of the SPP at a fixed angle will redshift as more target material is bound to the surface opposite of

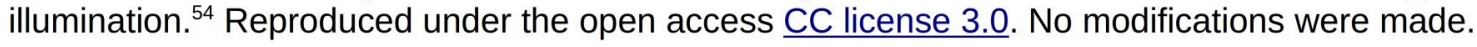

This SPR sensor works by altering the refractive index of the dielectric environment by selectively binding the molecule of choice to the back of the metal film in the flow channel. This effectively turn moves the SPR wavelength or angle of incidence which can be easily calibrated to give a range of working concentrations. Similarly, fiber-optic based systems, 
waveguides, and metal gratings can be used and reduced in size to micro- and nanostructured components. ${ }^{54}$ Another simple approach is to utilize coupling interactions between particles to yield an easily detectable far-field response. The surface modification of colloidal nanoparticles to induce selective aggregation when a specific molecule is introduced serves as a simple yet effective method for sensing. An excellent example for this is the use of gold nanoparticles and single stranded DNA. When the complimentary strand to connect the two is introduced, the DNA aggregates and causes inter-particle coupling and a strong colour change from red to blue. ${ }^{55}$

\subsubsection{Field Enhanced Spectroscopy}

The enhanced electric field that surrounds the particle when irradiated with resonant frequency light can be used to increase the signal of an emitter such as a fluorophore or Raman label that is located within the enhanced field. In the case of SERS, this works by providing the Raman label more electromagnetic energy to scatter, giving a stronger signal than in the absence of the enhanced field. The strength of the enhancement is given by the ratio of intensities between the Raman signal from the label with and without field enhancement. The high enhancement factors afforded by SERS has allowed Raman spectroscopy to achieve single molecule detection. The enhancement factor required to achieve this low limit is dependent on the label itself, often with enhancement factors on the order of $10^{7}$, while some molecules with lower cross sections require very high, but achievable enhancement factors of $10^{11}$ to achieve single molecule detection. ${ }^{56}$

Surface plasmons can also be used to increase the emission rate of fluorophores giving rise to surface enhanced fluorescence (SEF), with enhancement factors achievable on the order of around 30x. Although several orders of magnitude lower than the enhancement 
factors for SERS, single molecule detection is still achievable even in a complex chemical environments. ${ }^{54}$ The relative position of the fluorophore to the plasmonic material is more complicated than with Raman spectroscopy. In order to optimize fluorescence enhancement the radiative decay must be enhanced, while non-radiative channels need to be suppressed. Consequently the highest enhancement factor for SEF is often found when the label is placed

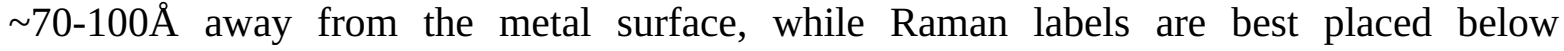
$30 \AA ̊{ }^{58}$ Conversely, utilizing the strongly distance dependent increase and then loss of signal for SEF can be useful as a nanoruler or sensor.

\subsubsection{Nanorulers}

One of the most challenging aspects of nanotechnology is quantifying events, objects, and distances that are below the diffraction limit making them inaccessible to conventional optics. As a result, several systems have been developed to act as nanorulers allowing researchers to easily take detailed measurements and yield information about distances that occur at the nanoscale. Nanorulers have already found application across the sciences, from materials and interfaces, to biology and biochemistry.

Plasmonic based nanorulers are particularly promising and becoming commonly used with some even achieving Ångström resolution. ${ }^{59}$ Nanometal surface energy transfer (NSET) for example, is often employed as a replacement to Förster Resonance Energy Transfer (FRET) in distance dependent biological studies due to its higher sensitivity and longer dynamic range. ${ }^{60}$ Many plasmonic nanorulers are based on a coupling interactions with an optical reporter, ${ }^{61}$ other particles, ${ }^{62}$ or an adjacent surface, ${ }^{63,64}$ and can use special properties such as Fano interferences for ultra-sensitive measurements. ${ }^{62}$ These configurations make the relative position(s) of the components of crucial importance, but in 
turn limits the application and complicates the task. Plasmonic nanoparticles can act as robust label free reporters by directly measuring the refractive index of something such as a thin film, but at a diminished sensitivity, resolution, and dynamic range compared to coupled methods. ${ }^{65}$

\subsubsection{Thermoplasmonics}

Thermoplasmonics utilizes the ability of plasmonic nanomaterials to efficiently generate heat from incident radiation, (Figure 1.11).
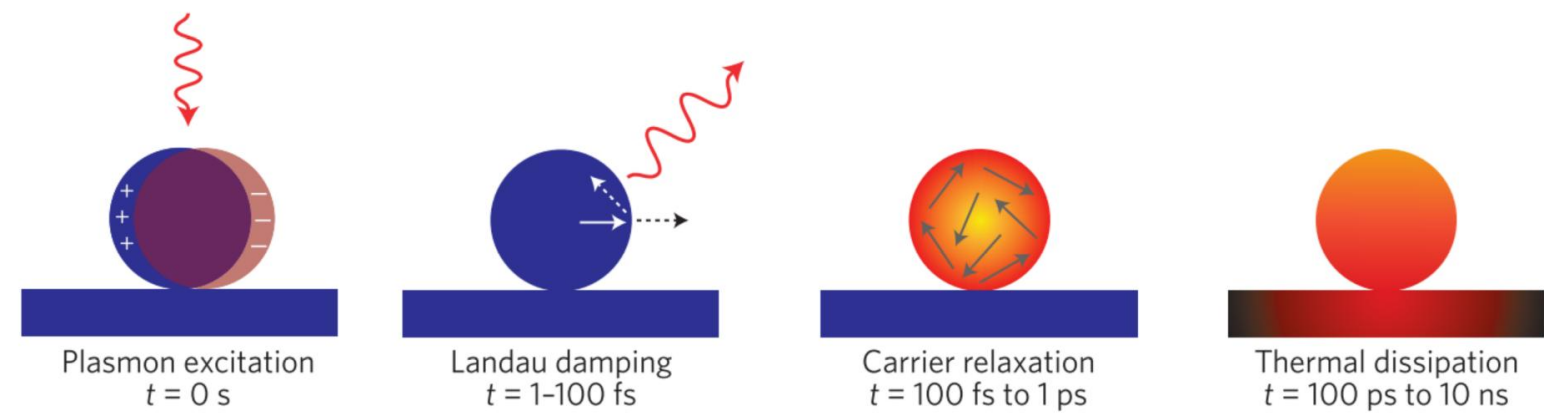

Figure 1.11: Schematic representation depicting the events that lead to thermal generation and dissipation beginning with the initial photoexitation of a plasmon. Reproduced with Permission. ${ }^{66}$ Copyright 2015 Nature Nanotechnology.

When excited, electrons oscillate in the conduction band of the particle, and as such are subject to internal resistance through collisions with the metal lattice producing heat. This causes the particles heat up rapidly, and allows for the delivery of thermal energy to their surroundings with nanoscale precision. ${ }^{66}$ This property has found use in applications such as cancer treatment and other thermal based biomedical applications, ${ }^{67,68}$ improved distillation that exceeds the azeotrope of ethanol-water mixtures, ${ }^{69}$ and desalinization of sea water. ${ }^{70}$

\subsubsection{Catalysis}

Plasmons can be used to drive chemical reactions in multiple pathways. Thermoplasmonics can be used to drive physical changes such as phase changes, but they 
can also be used to drive thermally activated chemical reactions, such as decomposition. The use of refractory plasmonics is allowing for the investigation of high temperature studies of thermally driven catalysis. ${ }^{24}$ Instead of relying on thermal activation, the electrons involved in a plasmon can also be used to directly drive chemical reactions, ${ }^{66}$ such as the hydrolysis of water, ${ }^{71}$ or the dissociation of hydrogen gas. ${ }^{72}$ This works by using a plasmon with the required energy to drive the reaction forward. Similarly, the enhanced electric field can be used to drive photocatalytic processes more efficiently than direct photocatalysis. ${ }^{73}$

\subsection{Shape Control of Silver Nanoparticles}

As the properties of plasmonic systems are so highly sensitive to the shape and size of the particles used, significant control over particle synthesis in terms of size, shape, and distribution is crucial. The polyol method, where a metal salt is heated in a simple hydroxylated hydrocarbon that acts as both the solvent and reducing agent is a robust method to selectively synthesize numerous particle geometries. The synthesis of silver nanocubes was first reported by Younan Xia in $2002^{74}$ using ethylene glycol (EG) in the presence of polyvinylpyrrolidone (PVP), which acts as a capping and structure directing agent. $^{75}$ There are several reaction pathways using EG as a reducing agent that occur at different temperatures, and sensitivities to the atmosphere. The dominant pathway is by reduction of the metal by glycolaldehyde (GA), which is produced from the oxidation of EG by dissolved $\mathrm{O}_{2}::^{76}$

$$
2 \mathrm{HOCH}_{2} \mathrm{CH}_{2} \mathrm{OH}+\mathrm{O}_{2} \rightarrow 2 \mathrm{HOCH}_{2} \mathrm{CHO}+2 \mathrm{H}_{2} \mathrm{O}
$$

Then reduction of silver by GA: 


$$
2 \mathrm{Ag}^{+}+2 \mathrm{HOCH}_{2} \mathrm{CHO} \rightarrow 2 \mathrm{Ag}^{0}+\mathrm{HOCH}_{2} \mathrm{COCOCH}_{2} \mathrm{OH}+2 \mathrm{H}^{+}
$$

GA is a stronger reducing agent than EG so the reaction rates and outcomes are highly sensitive to the concentration of GA present in the flask. To further complicate the scenario, silver nanoparticles catalyze reaction [1.1], increasing the rate of GA production once particle seeds have been formed. If the reaction proceeds under the protection of argon with no avenue for GA production, particles are still produced, but at a greatly diminished rate. ${ }^{76}$

Reduction of silver ions into metal nanoparticles can take many paths that will determine its final morphology. One of the necessary conditions to produce cubes is the growth of single crystal seeds. The degree of crystallinity is determined early on in the synthesis when stable seeds can coalesce into singly, or multiply twinned seeds, leading to different shapes of nanoparticles (Figure 1.12).

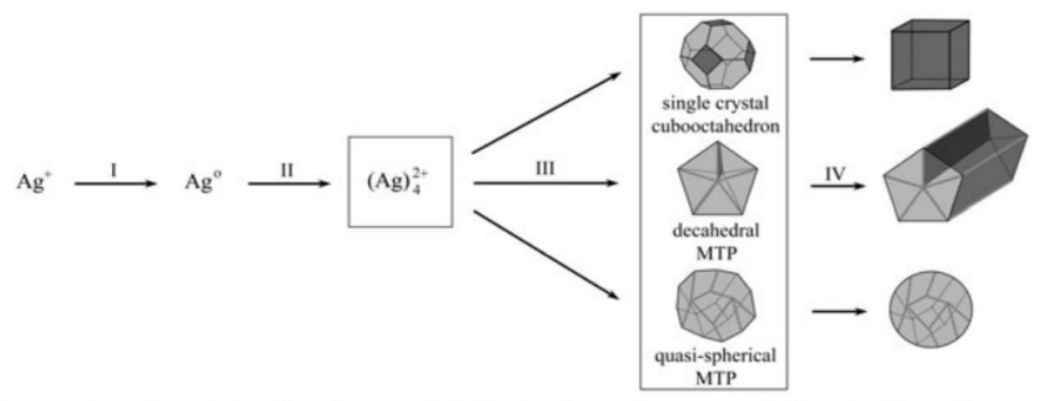

Figure 1.12: Schematic showing the fate of initial silver ions and illustrating the pathways towards different morphologies based on crystallinity of seeds produced in the reaction. Reproduced with Permission. ${ }^{75}$ Copyright John Wiley and Sons 2005.

Silver metal has face centered cubic lattice, and the $\{100\}$ facet has a higher energy than the close packed $\{111\}$ facet. This difference in energy promotes the production of twinned seeds and $\{111\}$ faceted single seeds, which take the shape of octahedrons. ${ }^{77,78}$ Just as the growth of silver on the $\{100\}$ facet is more energetically favorable, so is the adsorption of PVP, selectively blocking the $\{100\}$ facet, forcing silver growth along the $\{111\}$ facet. This 
relationship also lowers the rate at which twinned seeds are produced promoting the single crystal structure that nanocubes require. After single seeds are produced, nanoparticles continue to grow, but simply having single crystal seeds does not guarantee they grow into the desired shape. Spherical particles have the lowest total surface energy, but are made of up multiple low index facets. By protecting one facet and promoting growth on the other it is possible to yield particles that are dominated by either the $\{111\}$ facet, resulting in octahedrons, or the $\{100\}$ facet, resulting in cubes. This selectivity can be tuned by the relative abundance of silver and PVP available to the surface of the particle, ${ }^{78}$ or by selective etching of a particular face. ${ }^{79}$ This seeded technique can yield high quality octahedron and cube samples with a tunable edge lengths from $80-175 \mathrm{~nm}^{75,79}$

Several more methods have been developed to further suppress the formation of twinned seeds and during the synthesis. One strategy is the addition of trace amounts of sulfur to the reaction flask just prior to the injection of the silver precursor. ${ }^{8081}$ This leads to the formation of mono-disperse silver sulfide seeds at a rate that is significantly faster than the formation of silver seeds by GA reduction. The newly formed seeds act as a heteronucleation point for the growth of silver metal resulting it the generation of highly mono-disperse single crystal silver nanoparticles which then undergo structure directed growth by PVP to yield various shapes. The sulfur mediated method is faster, and can achieve a wider range of edge lengths for cubes down to 30nm. Another strategy to reduce the number of twinned seeds is to introduce an etching agent such as chloride, oxygen, or bromide. ${ }^{82-84}$ The addition of an etching agent preferentially dissolves twinned seeds, and small nanoparticles, which helps to ensure high mono-dispersity in samples with larger mean edge lengths. To further extend the range of possible edge lengths, the use of a weaker 
reducing agent, and a more stable silver source can be used to produce relatively small nanocubes. Diethylene glycol (DEG, $\left.\left(\mathrm{HOCH}_{2} \mathrm{CH}_{2}\right)_{2} \mathrm{O}\right)$, and silver trifluoroacetate $\left(\mathrm{CF}_{3} \mathrm{COOAg}\right)$ have bee used to produce AgNCs with controllable edge lengths from 18$32 \mathrm{~nm}^{28}$

\subsection{Rationale}

Solid supported silver nanocrystals provide a unique platform for the study and application of plasmonic properties in the visible region. This is due to the emergence of two spatially separated, tunable modes that occur when AgNCs are supported by a symmetry breaking dielectric. This thesis can be roughly divided into the characterization of these hybrid modes, and application of their properties beginning with the investigation into the refractive index sensitivities and spectral shifts that plasmonic modes experience in solid supported AgNCs.

Briefly, chapters 2 and 3 focus on the optical properties of supported AgNCs with edge lengths of 55-60nm by varying the RI of the substrate as well as the surrounding environment by submerging the films in varying solutions of water and EG. This allows for independent control over spectral positions of both hybrid modes, and quantification of their refractive index sensitives and figures of merit. The quadrupolar (Q) hybrid mode (located at the top of the cubes) attains a relatively high figure of merit for this region of the spectrum, while the dipolar (D) hybrid mode (located at the bottom of the cubes) has the highest sensitivity in terms of peak position.

Chapter 4 expands on these results by elucidating the impact of particle size on this relationship by performing the same experiments for substrates composed of AgNCs with edge lengths of 40, 80, and 130nm. This reveals that the hybrid Q mode is always the most 
sensitive regardless of the cube size in any particular system. The dependence on where the Q mode appears in the spectrum and its tunable range is also determined to be a function of both the size of the particles, and the RI of the underlying substrate. While the position of the Q mode is insensitive to the substrate, the maximum shift it can experience is limited by the position of the dipolar mode, which is determined by the RI of the substrate. This chapter also reinforces why cubes are superior to spheres for sensing, by showing that solid spheres support less observable plasmonic modes with lower refractive index sensitives and figures of merit when compared to cubes of a similar size.

Chapter 5 introduces the dependence on polarization and angle of incidence and its impact on spectral features. The Q mode was found to be strongly dependent on angle of incidence using p-polarized light, leading to a significant increase in intensity. No such relationship was found for the dipolar mode. This chapter represents a turning point in my research towards the application of the properties of solid supported AgNCs. Due to the highly tunable nature of this platform it can be utilized as an effective RI sensor for a wide range in the visible region, with high sensitivities and figures of merit. This also makes this an excellent platform for SERS as plasmon modes can be easily tuned to the desired wavelength for study.

Chapter 6 quantifies the spectral contributions of absorption and scattering in the visible region as functions of polarization and angles of incidence, including front and rear illumination geometries. The motivation for this work was to provide additional tools for spectral engineering that are not exclusively in terms of peak position. Strong anisotropy was found between illumination geometries, as well as a strong size dependence on the behavior of various modes was observed. Directional control over scattering, absorption, and 
transmission is applicable to the fabrication of optical materials with desired properties such as photovoltaic enhancement ${ }^{85}$ and projection displays. ${ }^{86}$

Chapter 7 develops the use of silver nanocubes as a nanoruler to measure the position of the cubes relative to an interface with a dynamic range of 60nm. This is achieved by utilizing the spatially resolved character of all the modes present in an ensemble of supported 75nm cubes. This configuration is significant as it does require an emitter or metal surface to couple with commonly seen in many plasmonic nanorulers. This greatly simplifies the application of this system and is used to explore the surface dynamics of a thin film of polystyrene just above its glass transition temperature. This nanoruler can be applied to any interface with moderate transparency in the visible region and allows the embedding of AgNCs into its surface and elucidate many surface properties, or simply used to quantify the position of the AgNCs within a matrix.

\subsection{References}

1 U. Leonhardt, Nat. Photonics, 2007, 1, 207-208.

2 J. Pérez-Pariente and A. B. Martín Rojo, J. Cult. Herit., 2008, 9, e81-e84.

3 M. Faraday, Philos. Trans. R. Soc. London, 1857, 147, 145-181.

4 H. Horvath, J. Quant. Spectrosc. Radiat. Transf., 2009, 110, 787-799.

5 G. Mie, Ann. Phys., 1908, 330, 377-445.

6 D. Pines and D. Bohm, Phys. Rev., 1952, 85, 338-353.

7 D. Pines, Rev. Mod. Phys., 1956, 28, 184-198.

8 L. Tonks and I. Langmuir, Phys. Rev., 1929, 34, 876-922.

9 R. H. Ritchie, Phys. Rev., 1957, 106, 874-881.

10 A. Otto, Zeitschrift fur Phys., 1968, 216, 398-410.

11 M. Fleischmann, P. J. Hendra and A. J. McQuillan, Chem. Phys. Lett., 1974, 26, 163166. 
12 D. L. Jeanmaire and R. P. Van Duyne, J. Electroanal. Chem. Interfacial Electrochem., 1977, 84, 1-20.

13 M. G. Albrecht and J. A. Creighton, J. Am. Chem. Soc., 1977, 99, 5215-5217.

14 P. Mulvaney, Langmuir, 1996, 12, 788-800.

15 M. Pelton and G. Bryant, Introduction to Metal-Nanoparticle Plasmonics, John Wiley \& Sons, Inc. and Science Wise Publishing, Hoboken, New Jersey, 2013.

16 W. L. Barnes, A. Dereux and T. W. Ebbesen, Nature, 2003, 424, 824-830.

17 T. R. Jensen, M. L. Duval, K. L. Kelly, A. A. Lazarides, G. C. Schatz and R. P. Van Duyne, J. Phys. Chem. B, 1999, 103, 9846-9853.

18 M. G. Blaber, M. D. Arnold and M. J. Ford, J. Phys. Condens. Matter, 2010, 22, 143201.

19 M. Rycenga, C. M. Cobley, J. Zeng, W. Li, C. H. Moran, Q. Zhang, D. Qin and Y. Xia, Chem. Rev., 2011, 111, 3669-3712.

20 K. M. Mayer and J. H. Hafner, Chem. Rev., 2011, 111, 3828-3857.

21 Y. H. Lee, H. Chen, Q.-H. Xu and J. Wang, J. Phys. Chem. C, 2011, 115, 7997-8004.

22 M. W. Knight, N. S. King, L. Liu, H. O. Everitt, P. Nordlander and N. J. Halas, ACS Nano, 2014, 8, 834-840.

23 G. V. Naik, V. M. Shalaev and A. Boltasseva, Adv. Mater., 2013, 25, 3264-3294.

24 U. Guler, A. Boltasseva and V. M. Shalaev, Science, 2014, 344, 263-264.

25 C. Noguez, J. Phys. Chem. C, 2007, 111, 3806-3819.

26 Y. Yang, S. Matsubara, L. Xiong, T. Hayakawa and M. Nogami, J. Phys. Chem. C, 2007, 111, 9095-9104.

27 D. Rossouw, M. Couillard, J. Vickery, E. Kumacheva and G. A. Botton, Nano Lett., 2011, 11, 1499-1504.

28 Y. Wang, Y. Zheng, C. Z. Huang and Y. Xia, J. Am. Chem. Soc., 2013, 135, 19411951.

29 E. Prodan, C. Radloff and Halas, Science, 2003, 302, 419-422.

30 S. J. Barrow, D. Rossouw, A. M. Funston, G. A. Botton and P. Mulvaney, Nano Lett., 2014, 14, 3799-3808. 
31 M. Liu, T.-W. Lee, S. K. Gray, P. Guyot-Sionnest and M. Pelton, Phys. Rev. Lett., 2009, 102, 107401.

32 F. Schertz, M. Schmelzeisen, R. Mohammadi, M. Kreiter, H. Elmers and G. Schönhense, Nano Lett., 2012, 12, 1885-1890.

33 P. Nordlander and E. Prodan, Nano Lett., 2004, 4, 2209-2213.

34 P. Nordlander, C. Oubre, E. Prodan, K. Li and M. I. Stockman, Nano Lett., 2004, 4, 899-903.

35 M. I. Stockman, Nature, 2010, 467, 541-542.

36 D. Solis, B. Willingham, S. L. Nauert, L. S. Slaughter, J. Olson, P. Swanglap, A. Paul, W. Chang and S. Link, 2012.

37 E. Ringe, J. M. McMahon, K. Sohn, C. Cobley, Y. Xia, J. Huang, G. C. Schatz, L. D. Marks and R. P. Van Duyne, J. Phys. Chem. C, 2010, 114, 12511-12516.

38 M. W. Knight, Y. Wu, J. B. Lassiter, P. Nordlander and N. J. Halas, Nano Lett., 2009, 9, 2188-2192.

39 P. Albella, B. Garcia-cueto, F. Gonz, F. Moreno, P. C. Wu, T. Kim, A. Brown, Y. Yang, H. O. Everitt and G. Videen, Nano Lett., 2011, 11, 3531-3537.

40 Y. Wu and P. Nordlander, J. Phys. Chem. C, 2010, 114, 7302-7307.

41 L. J. Sherry, S.-H. Chang, G. C. Schatz, R. P. Van Duyne, B. J. Wiley and Y. Xia, Nano Lett., 2005, 5, 2034-2038.

42 S. Zhang, K. Bao, N. J. Halas, H. Xu and P. Nordlander, Nano Lett., 2011, 11, 16571663.

43 U. Fano, Phys. Rev., 1961, 124, 1866-1878.

44 B. Luk'yanchuk, N. I. Zheludev, A. Maier, Stefan, N. J. Halas, P. Nordlander, H. Giessen and C. T. Chong, Nat. Mater., 2010, 9, 707-715.

45 G. Bachelier, I. Russier-Antoine, E. Benichou, C. Jonin, N. Del Fatti, F. Vallée and P.F. Brevet, Phys. Rev. Lett., 2008, 101, 197401.

46 O. Peña-Rodríguez and U. Pal, Nanoscale, 2011, 3, 3609.

47 A. Lovera, B. Gallinet, P. Nordlander and O. J. F. Martin, ACS Nano, 2013, 7, 45274536.

48 T. Nagatsuma, G. Ducournau and C. C. Renaud, Nat. Photonics, 2016, 10, 371-379. 
49 R. Stanley, Nat. Photonics, 2012, 6, 409-411.

50 T. König, R. Kodiyath, Z. A. Combs, M. A. Mahmoud, M. A. El-Sayed and V. V. Tsukruk, Part. Part. Syst. Charact., 2014, 31, 274-283.

51 V. Kravets, F. Schedin and a. Grigorenko, Phys. Rev. Lett., 2008, 101, 087403.

52 H. Chen, X. Kou, Z. Yang, W. Ni and J. Wang, Langmuir, 2008, 24, 5233-5237.

53 O. Kedem, A. Vaskevich and I. Rubinstein, Phys. Chem. Lett., 2011, 2, 1223-1226.

54 S. Roh, T. Chung and B. Lee, Sensors, 2011, 11, 1565-1588.

55 R. Elghanian, Science, 1997, 277, 1078-1081.

56 A. D. McFarland and R. P. Van Duyne, Nano Lett., 2003, 3, 1057-1062.

57 J. D. Flynn, B. L. Haas and J. S. Biteen, J. Phys. Chem. C, 2015, acs.jpcc.5b08049.

58 J. R. Lakowicz, C. D. Geddes, I. Grycznski, J. Malicka, Z. Grycznski, K. Aslan, E. Matveeva, J. Zhang, R. Badugu and J. Huang, J. Fluoresc., 2004, 14, 425-411.

59 B. Gallinet, T. Siegfried, H. Sigg, P. Nordlander and O. J. F. Martin, Nano Lett., 2013, 13, 497-503.

60 C. S. Yun, A. Javier, T. Jennings, M. Fisher, S. Hira, S. Peterson, B. Hopkins, N. O. Reich and G. F. Strouse, J. Am. Chem. Soc., 2005, 127, 3115-3119.

61 Y. Chen, M. B. O’Donoghue, Y.-F. Huang, H. Kang, J. a. Phillips, X. Chen, M.-C. Estevez, C. J. Yang and W. Tan, J. Am. Chem. Soc., 2010, 132, 16559-16570.

62 J. Butet and O. J. F. Martin, ACS Nano, 2014, 8, 4931-4939.

63 S. Shen, L.-Y. Meng, Y. Zhang, J. Han, Z. Ma, S. Hu, Y. He, J.-F. Li, B. Ren, T.-M. Shih, Z. Wang, Z.-L. Yang and Z.-Q. Tian, Nano Lett., 2015, 15, 6716-6721.

64 R. T. Hill, K. M. Kozek, A. Hucknall, D. R. Smith and A. Chilkoti, ACS Photonics, 2014, 1, 974-984.

65 P. D. Nallathamby, T. Huang and X.-H. N. Xu, Nanoscale, 2010, 2, 1715.

66 M. L. Brongersma, N. J. Halas and P. Nordlander, Nat. Nanotechnol., 2015, 10, 2534.

67 L. A. Dykman and N. G. Khlebtsov, Acta Naturae, 2011, 3, 34-55.

68 N. G. Khlebtsov and L. A. Dykman, J. Quant. Spectrosc. Radiat. Transf., 2010, 111, 1-35. 
69 O. Neumann, A. S. Urban, J. Day, S. Lal, P. Nordlander and N. J. Halas, ACS Nano, 2013, 7, 42-49.

70 L. Zhou, Y. Tan, J. Wang, W. Xu, Y. Yuan, W. Cai, S. Zhu and J. Zhu, Nat. Photonics, 2016, 10, 393-398.

71 H. Robatjazi, S. M. Bahauddin, C. Doiron and I. Thomann, Nano Lett., 2015, 15, 6155-6161.

72 S. Mukherjee, F. Libisch, N. Large, O. Neumann, L. V Brown, J. Cheng, J. B. Lassiter, E. a Carter, P. Nordlander and N. J. Halas, Nano Lett., 2013, 13, 240-247.

73 X. Meng, L. Liu, S. Ouyang, H. Xu, D. Wang, N. Zhao and J. Ye, Adv. Mater., 2016.

74 Y. Sun, Science, 2002, 298, 2176-2179.

75 B. Wiley, Y. Sun, B. Mayers and Y. Xia, Chem. - A Eur. J., 2005, 11, 454-463.

76 S. E. Skrabalak, B. J. Wiley, M. Kim, E. V. Formo and Y. Xia, Nano Lett., 2008, 8, 2077-2081.

77 X. Xia, J. Zeng, L. K. Oetjen, Q. Li and Y. Xia, J. Am. Chem. Soc., 2012, 134, 17931801.

78 X. Xia and Y. Xia, Nano Lett., 2012, 12, 6038-6042.

79 Y. Wang, D. Wan, S. Xie, X. Xia, C. Z. Huang and Y. Xia, ACS Nano, 2013, 7, 45864594.

80 A. R. Siekkinen, J. M. McLellan, J. Chen and Y. Xia, Chem. Phys. Lett., 2006, 432, 491-496.

81 Q. Zhang, C. Cobley, L. Au, M. McKiernan, A. Schwartz, L.-P. Wen, J. Chen and Y. Xia, ACS Appl. Mater. Interfaces, 2009, 1, 2044-2048.

82 S. H. Im, Y. T. Lee, B. Wiley and Y. Xia, Angew. Chemie, 2005, 44, 2154-2157.

83 B. Wiley, T. Herricks, Y. Sun and Y. Xia, Nano Lett., 2004, 4, 1733-1739.

84 D. Yu and V. W. Yam, J. Am. Chem. Soc., 2004, 126, 13200-13201.

85 R. Santbergen, T. L. Temple, R. Liang, A. H. M. Smets, R. A. C. M. M. Van Swaaij and M. Zeman, J. Opt., 2012, 14.

86 K. Saito and T. Tatsuma, Nanoscale, 2015, 7, 20365-20368.

87 D. Prezgot, Masters Thesis, Department of Chemistry, Carleton University, 2014. 


\section{Chapter 2}

Plasmonic properties of silver nanocube monolayers on high refractive index substrates 
Modified from original manuscript published as:

Bottomley, A., Prezgot, D. and Ianoul, A., Plasmonic properties of silver nanocube monolayers on high refractive index substrates. Applied Physics A 2012, 109, 869-872. doi:10.1007/s00339-012-7354-z

Department of Chemistry, Carleton University, 1125 Colonel By Drive, Ottawa, Ontario, Canada, K1S 5B6

\subsection{Abstract}

Extinction spectra of nanocubes supported by a symmetry breaking dielectric substrate are very different from those in solution. In this work, we varied the refractive index of the substrate in order to optimize the refractive index sensitivity (RIS) of supported silver nanocube monolayers. We found that on thin (5-7nm) silicon films, the RIS is characterized by the figure of merit (FOM) for the quadrupolar plasmonic mode as high as 5.0, making silicon supported silver nanocube monolayers a promising sensing platform.

\subsection{Introduction}

Localized surface plasmon resonances (LSPR) of noble metal nanoparticles have been studied intensely in recent years. The ability to control and tune LSPRs is crucial to both understanding and application in the field of plasmonics ${ }^{1}$. Progress made in fabrication methods ${ }^{2}$ and theory for metal nanoparticles has allowed for development of novel optical and sensing materials ${ }^{3}$. When the LSPRs of these particles are excited, a strong enhancement of their local electric fields occurs at the surface of the particles. This field enhancement allows for increased signal from optical techniques such as $\operatorname{Raman}^{4,5}, \mathrm{IR}^{6}$, and fluorescence ${ }^{7-}$ ${ }^{10}$ spectroscopy. The spectral extinction spectra of LSPRs are also very sensitive to the refractive index of their local environment making them an excellent platform for sensing. ${ }^{3,11}$ The plasmonic signature of metal nanostructures can be influenced in several ways by modifying their size, shape, and material, ${ }^{1,12}$ but also through interparticle interactions ${ }^{13}$ and 
particle substrate interactions. ${ }^{14,15}$ Such investigations have shown unique properties such as collective modes and Fano-like resonances. ${ }^{16}$ Plasmonic signatures of nanoparticles that are supported by a dielectric material exhibit significant changes in their LSPRs when compared to particles in colloidal solutions. The most notable change is the splitting of a single dipolar plasmonic mode into two distinct modes. ${ }^{16-18}$ When dealing with spherical nanoparticles, these two new modes correspond to dipolar oscillations with orthogonal polarizations. ${ }^{18}$ However, when nanocubes are deposited on a dielectric support, their substrate interaction leads to plasmon mode hybridization, resulting in two distinct bands with corresponding charge oscillations located at the opposite sides of the nanocube. ${ }^{14,16-18}$ Separation between the bands in the case of cubes was found to be greater than that for the spherical particles dependent on the size,$^{17}$ edge, and corner sharpness, as well as distance from the substrate. ${ }^{19}$ Each of these hybrid bands present in the nanocubes is sensitive to the refractive index of their respective environments, one being the substrate and the other the surrounding medium in which the supported layer is immersed. ${ }^{14,16,17}$ In the present work, we fabricated quasiperiodic arrays of silver nanocubes on substrates with varying refractive indices. This allowed for a series of refractive index sensitivity measurements that relate the changes in refractive index of the substrate to changes in the surrounding medium.

\subsection{Experimental}

\section{Chemicals}

Silver nitrate 99+\%, polyvinylpyrrolidone (PVP) with a molecular weight of 55000, anhydrous 99.8\% ethylene glycol (EG), anhydrous sodium sulfide, and chloroform were purchased from Sigma-Aldrich and used as obtained. 1,2-dioleoyl-sn-glycero-3phosphocholine (DOPC) was purchased from Avanti Polar Lipids, Alabaster, AL. Ethanol (95\%) was obtained from Commercial Alcohols and used without further purification. 


\section{Preparation of Nanocubes}

Silver nanocubes (NC) with an edge length of 55nm were synthesized according to the procedure described in the literature. ${ }^{20}$ Typically, a $100 \mathrm{~mL}$ round bottom flask containing $35 \mathrm{~mL}$ of EG and a clean stir bar were placed in an oil bath set to $150^{\circ} \mathrm{C}$ and allowed to heat for 1 h. Using a glass syringe, $5 \mathrm{~mL}$ of EG containing $0.4 \mathrm{~g}$ of dissolved PVP was injected. Five minutes later, using a micropipette $400 \mu \mathrm{L}$ of $3 \mathrm{mM}$ sodium sulfide dissolved in EG was added. After an additional $5 \mathrm{~min}, 2.5 \mathrm{~mL}$ of $282 \mathrm{mM}$ silver nitrate dissolved in EG was injected slowly using a glass syringe. Upon addition of the silver nitrate, the solution immediately turned black, slowly became a transparent yellow, and then changed to an ochre colour while some plating in the flask occurred. The reaction was monitored by periodically taking small aliquots out of the reaction flask using a pasture pipette and dispersing it in a cuvette filled with ethanol for UV- visible spectroscopy. The reaction was quenched by placing the flask in an ice bath when the appearance of a small but distinct peak at 350nm was observed in the spectra, indicating a cubic structure was present.

\section{Purification of Colloidal Silver Nanocubes}

The as-synthesized colloidal nanocubes contained unreacted EG, and an excess of PVP. EG and PVP were removed by diluting the colloidal solution with ethanol (1:1 by volume) followed by centrifugation at $12,000 \mathrm{rpm}$. The precipitate was collected and redispersed in ethanol by sonication. This process was repeated 30 times for complete removal of EG and PVP. The purified silver NC solution was further subjected to washing with chloroform by centrifugation (at least 3 times) prior to LB film preparation. The final volume of the nanocube solution was $4 \mathrm{~mL}$.

\section{Preparation of LB monolayers}

Nanocube substrates were prepared according to the procedure reported previously. ${ }^{21}$ 
Briefly, a NIMA 311D trough, filled with Milli-Q water $(18.2 \mathrm{~m} \Omega \mathrm{cm})$, was used to pre- pare the Langmuir monolayers of different NC samples. In order to form monolayers, an appropriate amount of each sample was deposited onto the water surface using a micro syringe. Each monolayer was left for 20min to allow chloroform evaporation. The surface pressure of the monolayer was measured with a paper Wilhelmy plate. Before transferring the monolayer onto solid supports, several isotherm cycles were performed to anneal the films to surface pressures in the solid phase. Monolayers of nanocube/DOPC mixtures were transferred onto substrates cleaned with aqua regia, and subsequently with mixture of chloroform and methanol at various surface pressures by vertical upward dipping at $2 \mathrm{~mm} / \mathrm{min}$ at room temperature.

\section{UV-vis Measurements}

UV-vis spectra of monolayers deposited on the substrates were recorded using a Shimadzu UV-2450 UV-vis spectrophotometer. Spectra were analyzed using GRAMS/AI spectral data processing software. Spectral deconvolution was performed using an appropriate number of mixed Gaussian-Lorentzian functions as well as a linear baseline to achieve the best fit to the original trace while minimizing $x^{2}$ values. To determine the refractive index sensitivities (RIS), the monolayer slides were immersed in water/EG solutions with varying volume percentages and the extinction spectra measured. The refractive indices of the mixtures were calculated from the volume percentages of the ingredients according to the Lorentz-Lorenz equation. ${ }^{22}$ The refractive indices of the pure solvents were 1.3334 and 1.4318 , for water and EG, respectively. ${ }^{22}$ The plasmon shifts were plotted as a function of the refractive index. The refractive index sensitivities were obtained from the slope of the linear fitting. 


\section{Topographical measurements}

The topography of the NC monolayers, transferred onto substrates at different surface pressures, was obtained using an Ntegra (NTMDT, Russia) atomic force microscope in semicontact mode in air at $23^{\circ} \mathrm{C}$ with $512 \times 512$ points per image. A $100 \times 100 \mu \mathrm{m}^{2}$ scanner (Ntegra) and cantilevers with rotated monolithic silicon tips $(125 \mu \mathrm{m}$ long, $40 \mathrm{~N} / \mathrm{m}$ spring constant Tap 300Al, resonance frequency $315 \mathrm{kHz}$, Budget Sensors) were used for all topographic measurements. The typical scan rate was $0.5 \mathrm{~Hz}$. AFM images were further processed by Nova image processing software.

\subsection{Results and Discussion}

Colloidal silver nanocubes of $\sim 55 \mathrm{~nm}$ edge size used in the present study are characterized by an extinction spectrum with a single dipolar LSPR peak at 443nm, and a small peak at 350nm. ${ }^{23}$ Applications exploiting plasmonic properties often require nanoparticles to be deposited on a solid substrate. We have previously shown that Langmuir Blodgett monolayer technique allows not only effective deposition, but also control of plasmonic properties of monolayers of silver nanocubes. ${ }^{14,15,17}$ Such monolayers are proven to be effective in enhancing surface enhanced Raman scattering (SERS) signal, but also in probing refractive index change in the environment and, therefore, represent a powerful sensing platform. Plasmonic properties of supported by a dielectric nanocubes, however, differ significantly from those in a suspension., ${ }^{1,34,23}$ LSPR-induced charges in such dielectric substrates disturb the nanocube electric field leading to plasmon mode hybridization with the appearance of two new bands: dipolar (D) and quadrupolar (Q). The charge oscillations for these new bands are located at the opposite sides of the cube: dipolar

near the substrate and quadrupolar away from the substrate. ${ }^{1,3,14,23}$ The degree of spectral separation between the two bands and the refractive index sensitivity depend on the distance 
between the substrate and the cubes, contact area, and the refractive index $\left(n_{\mathrm{RI}}\right)$ of the substrate (Fig. 2.1.) $)^{1,14,23}$

In this work, we prepared a monolayer of $55 \mathrm{~nm}$ silver nanocubes on several substrates with varying refractive indices. The samples were found to be very homogeneous with very uniform nanocubes (Fig. 2.1a). In addition, to minimize interparticle dipolar coupling, the monolayer was prepared from a mixture of nanocubes and a phospholipid DOPC. We have previously shown that by using mixtures consisting of nanocubes and lipids of different kind it is possible to pattern the nanocubes in monolayers. Depending on the lipid properties, we were able to obtain homogeneously dispersed nanocubes, as shown in Fig. 2.1a. It turns out that in such monolayers separation between the nanocubes was maintained at $\sim 130 \mathrm{~nm}$ (Fig. 2.1b). As a result, extinction spectra of supported nanocubes contained only small long wave- length peaks corresponding to dipole-dipole coupling between adjacent cubes, observed at Fig. 2.1c in the 550-700nm spectral region. We used four substrates with increasing refractive indexes: glass, sapphire, 5nm Si film deposited on glass, and a 7nm Si film deposited on glass. Due to plasmonic band hybridization, two bands visible in the spectra are observed and assigned to Q and D modes. 


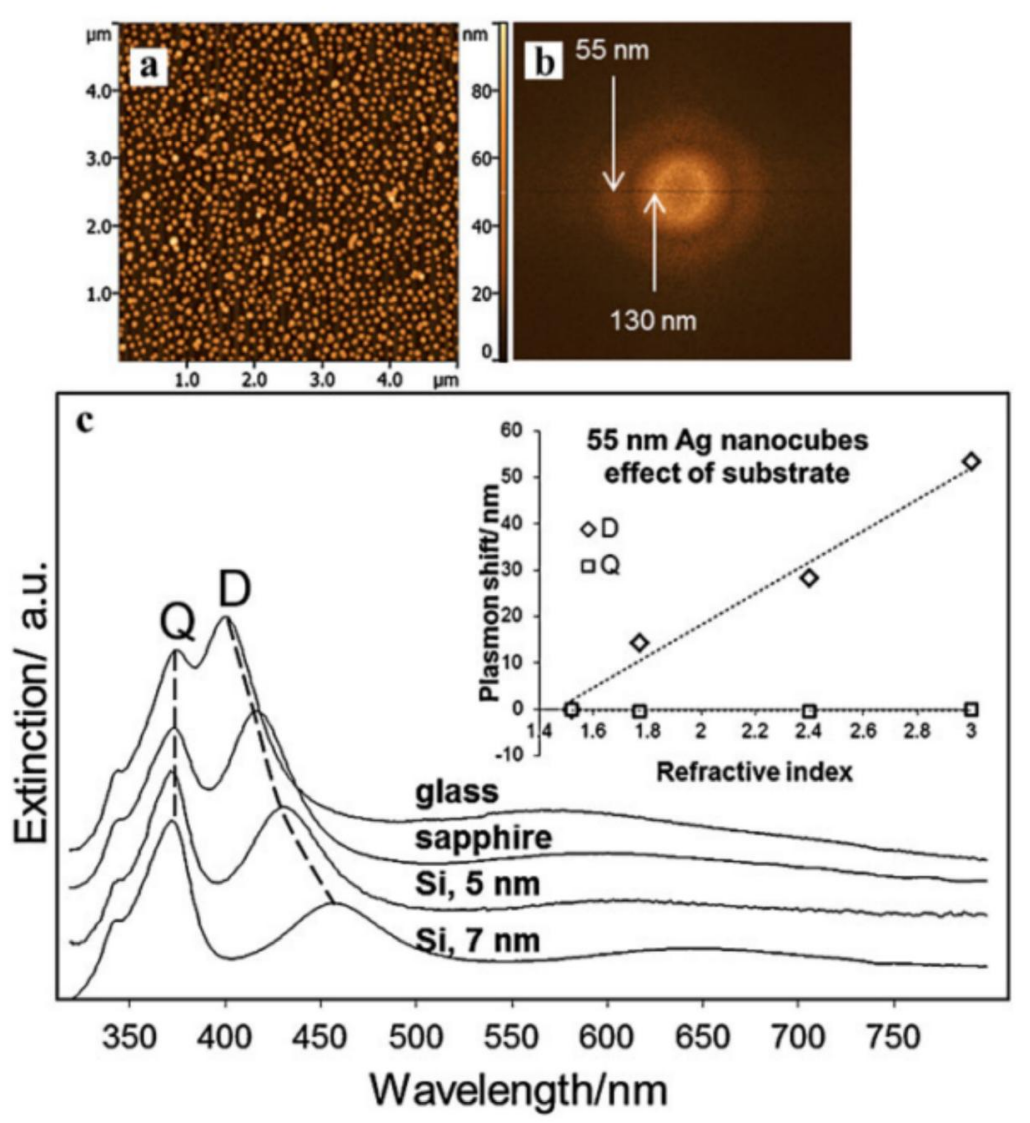

Figure 2.1. Supported silver nanocube monolayer prepared by Langmuir Blodgett technique: (a) AFM topography and (b) Fast Fourier transform (FFT) images indicate the size (55nm) and the average interparticle distance (130nm); (c) extinction spectra of supported silver nanocube monolayers indicate strong dependence of the dipolar (D) but not the quadrupolar (Q) mode on the substrate refractive index.

Since charge oscillations corresponding to the D mode are located near the substrate surface the dipolar band shows strong dependence on the substrate refractive index, and redshifts by more than 50nm when the refractive index of the substrate changes from 1.52 for glass to $\sim 3.0$ for a $7 \mathrm{~nm}$ silicon film (Fig. 2.1c inset). In contrast, the quadrupolar mode is insensitive to the substrate refractive index. 


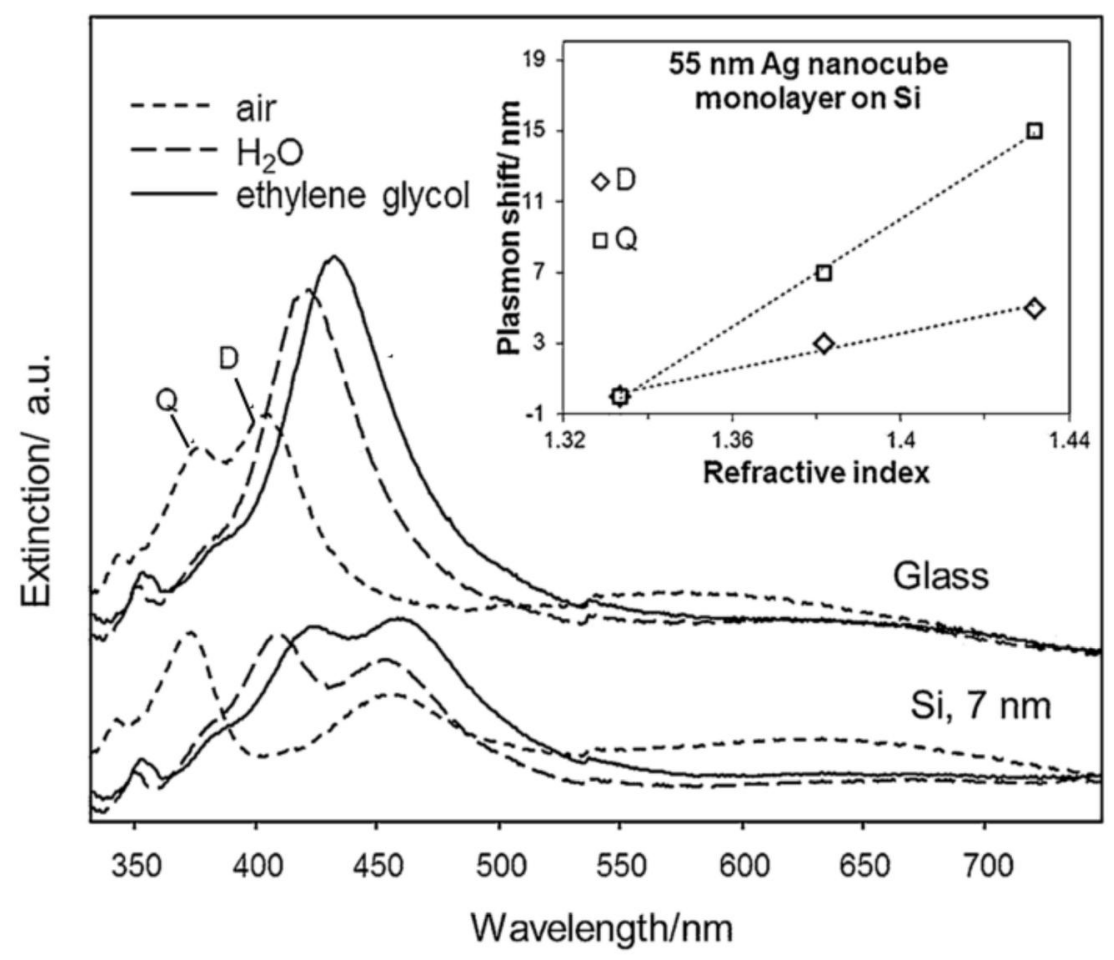

Figure 2.2. Solution refractive index sensing using nanocube monolayers deposited on glass and on a $7 \mathrm{~nm}$ thick silicon film. Inset shows solution refractive index dependencies of the dipolar (D) and quadrupolar (Q) peaks

At the same time, both modes were found to be sensitive to the refractive index change of the medium (Fig. 2.2). And, since change oscillations corresponding to the $\mathrm{Q}$ mode are located at the nanocube side away from the substrate, it was found to have a stronger dependence than the D mode (Fig. 2.2 inset). ${ }^{14,23}$ At the same time, the nature of the supporting dielectric substrates appears to play a crucial role in our ability to track spectral changes associated with the medium refractive index change. For example, when $n_{\mathrm{RI}}$ of the substrates is relatively low, as for glass, where $n_{\mathrm{RI}}=1.52$, upon immersion in liquid plasmon mode hybridization disappears (Fig. 2.2). This is due to insufficiently large refractive index change between the substrate and the medium to induce plasmon mode hybridization. As a result, plasmonic signature of glass supported silver nanocube monolayer immersed in water resembles that of colloidal nanocubes, mentioned before. The main band observed in such monolayer corresponds to dipolar oscillations and show modest sensitivity to the medium 
refractive index (Fig. 2.2). At the same time, if the substrate has a sufficiently high $n_{\mathrm{RI}}$, as with the $7 \mathrm{~nm}$ silicon film $(n \sim 3.0)$, the dipolar and quadrupolar bands remain clearly distinguished even when submerged in high refractive index liquids, such as ethylene glycol (Fig. 2.2). The $\mathrm{Q}$ and $\mathrm{D}$ bands remain well separated in various solutions: pure water and ethylene glycol. By tracking the position of each band in solvents with increasing $n_{\mathrm{RI}}$, we were able to determine the refractive index sensitivity (RIS) of the quadrupolar band to be 150 refractive index units/nm, which is about twice as high as for the D mode (Fig. 2.2 inset). Another parameter that is often used in evaluating sensitivity of various platforms to the refractive index change, namely the figure of merit (FOM), was introduced as a ratio of RIS to the full width at half maximum for a band. From the data obtained in this work, we were able to obtain the figure of merit for the quadrupolar band as high as $\sim 5.0$. This value is one of the highest reported so far for plasmonic nanostructures.

\subsection{Conclusions}

In this work, we prepared monolayers of silver nanocubes on substrates with various refractive indexes in order to enhance the sensitivity of such plasmonic materials to the refractive index change. We found that by changing the refractive index of the substrate dipolar and quadrupolar plasmon mode hybridization can be carefully adjusted and finetuned. As a result, silicon supported silver nanocube monolayers are shown to represent a powerful platform for sensing the refractive index change.

\subsection{References}

1 M. Rycenga, C.M. Cobley, J. Zeng,W. Li, C.H. Moran, Q. Zhang, D. Qin, Y. Xia, Chem. Rev. 111, 3669-3712 (2011)

2 M. Jones, K. Osberg, R. Macfarlane, M. Langille, C. Mirkin, Chem. Rev. 111, 37363827 (2011)

3 K. Mayer, J. Hafner, Chem. Rev. 111, 3828-3857 (2011) 
$4 \quad$ P.C. Lee, D. Meisel, J. Phys. Chem. 86, 3391-3395 (1982)

5 M. Fleischmann, P.J. Hendra, A.J. McQuillan, Chem. Phys. Lett. 26, 163-166 (1974)

6 A. Hartstein, J.R. Kirtley, J.C. Tsang, Phys.Rev. Lett. 45, 201-204 (1980)

$7 \quad$ A. Ianoul, A. Bergeron, Langmuir 22, 10217-10222 (2006)

8 A. Parfenov, I. Gryczynski, J.Malicka, C. Geddes, J.R. Lakowicz, J. Phys. Chem. B 107, 8829-8833 (2003)

$9 \quad$ P. Anger, P. Bharadwaj, L. Novotny, Phys. Rev. Lett. 96, 113002 (2006)

10 H. Rigneault, J. Capoulade, J. Dintinger, J. Wenger, N. Bonod, E. Popov, T.W. Ebbesen, P.F. Lenne, Phys. Rev. Lett. 95, 117401 (2005)

11 Y. Lee, H. Chen, Q. Xu, J. Wang, J. Phys. Chem. C 115, 7997- 8004 (2011)

12 C. Noguez, J. Phys. Chem. C 111, 3806-3819 (2007)

13 P. Albella, B. Garcia-Cueto, F.G. Alez, F. Moreno, P.Wu, T. Kim, A. Brown, Y.Yang, H. Everitt, G.Videen, Nano Lett. 11(9), 3531- 3537 (2011)

14 N. Ahamad, A. Bottomley, A. Ianoul, J. Phys. Chem. C 116, 185- 192 (2012)

15 N. Ahamad,D. Prezgot,A. Ianoul, J.Nanoparticle Res. 14(2), 724 (2012)

16 S. Zhang, K. Bao, N. Halas, H. Xu, P. Nordlander, Nano Lett. 11, 1657-1663 (2011)

17 A. Bottomley, A. Ianoul, ChemPhysChem 12, 2912-2914 (2011)

18 M.W. Knight, Y.Wu, J.B. Lassiter, P. Nordlander, N. Halas, Nano Lett. 9, 2188-2192 (2009)

19 E. Ringe, J. McMahon, K. Sohn, C. Cobley, Y. Xia, J. Huang, G. Schatz, L.Marks, R.Van Duyne, J. Phys. Chem. C 114, 12511- 12516 (2010)

20 M.A. Mahmoud, M.A. El-Sayed, Nano Lett. 11, 946-953 (2011)

21 N. Ahamad, A. Ianoul, J. Phys. Chem. C 115, 3587-3594 (2011)

22 H. Chem, X. Kou, Z. Yang,W. Ni, J.Wang, Langmuir 24, 5233- 5237 (2008)

23 L. Sherry, S. Chang, G. Schatz, R. Van Duyne, B. Wiley, Y. Xia, Nano Lett. 5, 2034 2038 (2005) 


\section{Chapter 3}

Improved Refractive Index Sensitivity of Silver Nanocube Monolayers on Silicon Films

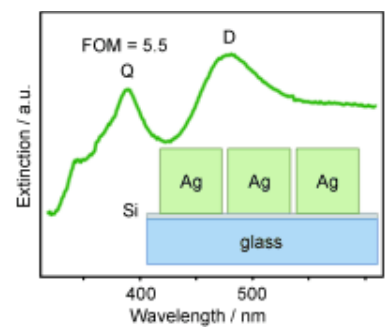


Modified from original manuscript published as:

Bottomley, A. and Ianoul, A., Improved Refractive-Index Sensitivity of Silver-Nanocube Monolayers on Silicon Films. ChemPhysChem 2011, 12, 2912-2914.

doi:10.1002/cphc.201100626

Department of Chemistry, Carleton University, 1125 Colonel By Drive, Ottawa, Ontario, Canada, K1S 5B6

\subsection{Abstract}

Suitable substrates: An improvement in the refractive-index sensitivity is observed for the quadrupolar plasmonic mode of a monolayer of $60 \mathrm{~nm}$ silver nanocubes deposited on a thin silicon film. Dipolar plasmonic band splitting on this high-refractive-index substrate is stronger than on a glass substrate. As a result, the quadrupolar band is easier to identify, even in high-refractive-index liquids. The work demonstrates the importance of using highrefractive-index substrates.

\subsection{Results and Discussion}

Localized surface plasmon resonances (LSPR) in silver nanocubes have been of interest due to their high sensitivity with respect to the local refractive-index change, ${ }^{1-4}$ particularly useful in sensors. ${ }^{5}$ The plasmonic signature of nanocubes supported by a symmetry breaking dielectric substrate is very different from that in solution. LSPR-induced charges in such dielectric substrates disturb the nanocube electric field, leading to splitting of a single dipolar plasmonic mode into two modes: dipolar (D), associated with charge oscillations at the nanocube side near the substrate, and quadrupolar (Q), corresponding to charge oscillations at the nanocube side away from the substrate. ${ }^{1-3,6}$ The degree of splitting and the refractive index sensitivity (RIS) depend on size, ${ }^{6}$ edge sharpness, ${ }^{1}$ separation from the substrate, ${ }^{1,6}$ and refractive index of the substrate. ${ }^{3}$ The $\mathrm{Q}$ band shows a much stronger dependence on the refractive index of the medium than the original dipolar band of colloidal 
nanocubes. ${ }^{1-3}$ Detailed investigations of the band splitting have been carried out using computational models, ${ }^{3}$ as well as experimental techniques, at a single supported nanoparticle level. ${ }^{1,2}$ However, for routine and consistent measurements, it is important to develop substrates that can be easily and reproducibly fabricated. ${ }^{7,8}$ One of the main drawbacks of ensemble compared to the single nanoparticle measurements is a usually larger half width of the plasmonic peaks, impeding identification and monitoring of the $\mathrm{D}$ and Q modes. Herein, we demonstrate that when a substrate with a sufficiently high refractive index, such as a silicon film, is used and the nanocube sample is fairly monodisperse, efficient peak splitting can be realized even for a monolayer with the resulting refractiveindex sensing parameters comparable to those of a single nanoparticle.

Figure 3.1 presents the UV/Vis extinction spectra of the silver nanocubes studied herein. Spectrum C corresponds to a suspension of 60nm nanocubes in ethanol, spectrum B to a Langmuir-Blodgett monolayer deposited on a quartz substrate (nRI $=1.44$ ) at low surface pressure corresponding to the gas phase of nanocubes, ${ }^{9}$ and spectrum $\mathrm{A}$ to a monolayer deposited on a glass substrate coated with a thin silicon film. A thickness of 5nm was chosen for the thin film to maintain substrate transparency in the visible region for the UV/Vis measurements. The resulting refractive index of such system was measured to be 2.4, lower than that of bulk silicon $(\sim 4.0){ }^{10}$ 


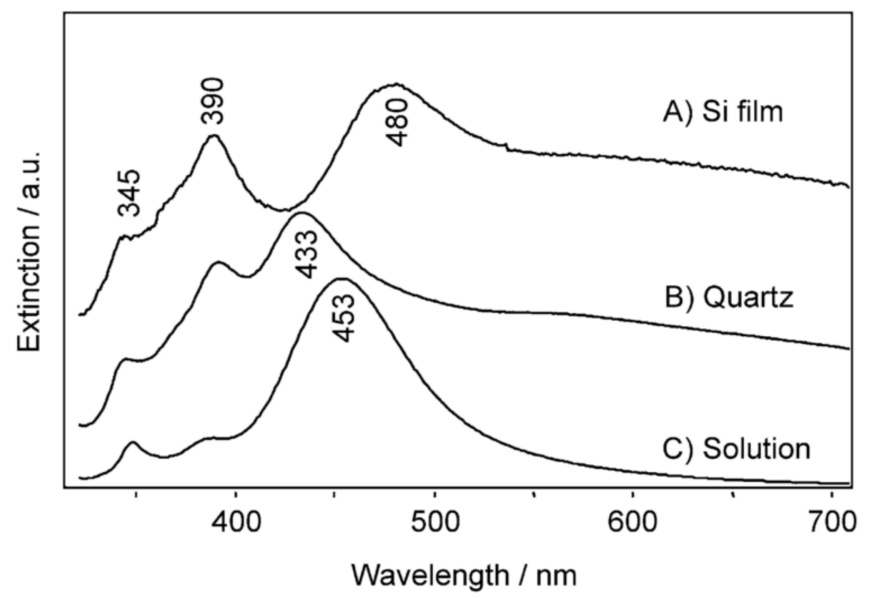

Figure 3.1. UV/Vis spectra of the Ag nanocubes: A) Monolayer deposited on a thin Si film. B) Monolayer deposited on quartz C) Suspension in $\mathrm{EtOH}$

The solution spectrum contains three peaks: A dipolar peak located at 453nm and two other peaks at 390 and 350nm, likely corresponding to quadrupolar and octupolar modes. ${ }^{1,4,11,12}$ The full-width-at-half maximum (FWHM) for the dipolar peak is $\sim 60 \mathrm{~nm}$. The nanocube edge length (of $60 \pm 5 \mathrm{~nm}$ ) and the size distribution were determined using atomic force microscopy. When the nanocubes are deposited on a quartz substrate, the dipolar mode splits into dipolar and quadrupolar at 433 and 390nm, respectively (Figure 3.1B). The longwavelength band at 550nm corresponds to the dipole-dipole coupling between adjacent nanoparticles. ${ }^{9}$ When a monolayer of the same pressure is transferred onto a silicon film, the two bands appear at 480 and 390nm, respectively (Figure 3.1A). The shift of the D peak to longer wavelength values is consistent with the higher refractive index of the silicon film compared to quartz. ${ }^{1-3}$ The position of the $\mathrm{Q}$ band is, however, fairly constant, nearly independent of the substrate refractive index.

We found that when a monolayer of silver nanoparticles is immersed in a liquid, the separation between the $\mathrm{D}$ and $\mathrm{Q}$ peaks decreases. If the difference between the refractive indices of the substrate and the solution is less than 0.6, then the two peaks are very difficult to separate. This could be seen when silver nanocubes deposited on the quartz substrate were 
immersed in water. The resulting extinction spectrum contained a single dipolar band at approximately 440nm as the refractive-index difference between quartz and water was only about 0.1 . On the other hand, when the monolayer of silver nanocubes deposited on a silicon film was immersed in water, the spectral separation between the quadrupolar and the dipolar peaks decreased but remained sufficiently large, allowing both peaks to be distinguished (Figure 3.2A).

The separation decreases when the substrate is immersed in liquids with higher refractive indices, such as ethylene glycol (Figure 3.2B). However, even in such cases, the D and Q bands can be distinguished. By performing spectral deconvolution of the extinction spectra, the dipolar and quadrupolar modes can be clearly identified as peaks 3 and 2, respectively.

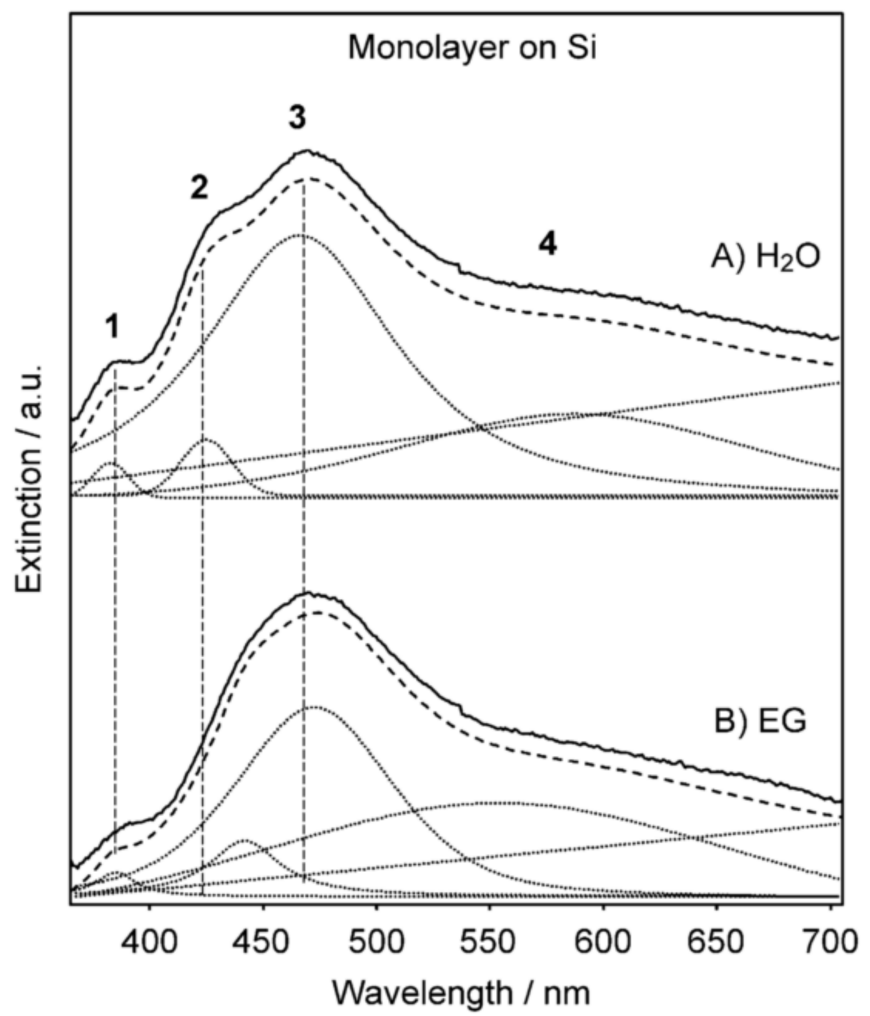

Figure 3.2. UV/Vis spectra of a monolayer of Ag nanocubes deposited on a Si film immersed in: A) water, and B) ethylene glycol. The solid curves (-) represent the experimental spectra, the dashed curves (----) show the results of the spectral deconvolution, and the dotted curves ( $\cdots \bullet$ ) represent individual peaks. 
With increasing refractive index of the solution, the dipolar peak red-shifted by $6 \mathrm{~nm}$, whereas the quadrupolar peak shifted by $\sim 18 \mathrm{~nm}$ (Figure 3.3). The calculated RIS (determined as the slope of the linear fitting) are 78 and $170 \mathrm{~nm} / \mathrm{RIU}$ for the dipolar and quadrupolar peaks, respectively. The respective figures of merit are 0.9 and 5.5.

The figure of merit determined here for the Q mode is one of the highest reported so far for measurements of ensemble of supported nanoparticles, ${ }^{5}$ and is comparable to that obtained for single nanocubes. ${ }^{2}$ This can be rationalized by the fact that the LSPR maximum of the Q peak does not significantly depend on the side length of the nanocubes. ${ }^{6}$ As a result, the sensitivity (even that of a heterogeneous collection of nanocubes) can be as high as that of a single nanoparticle.

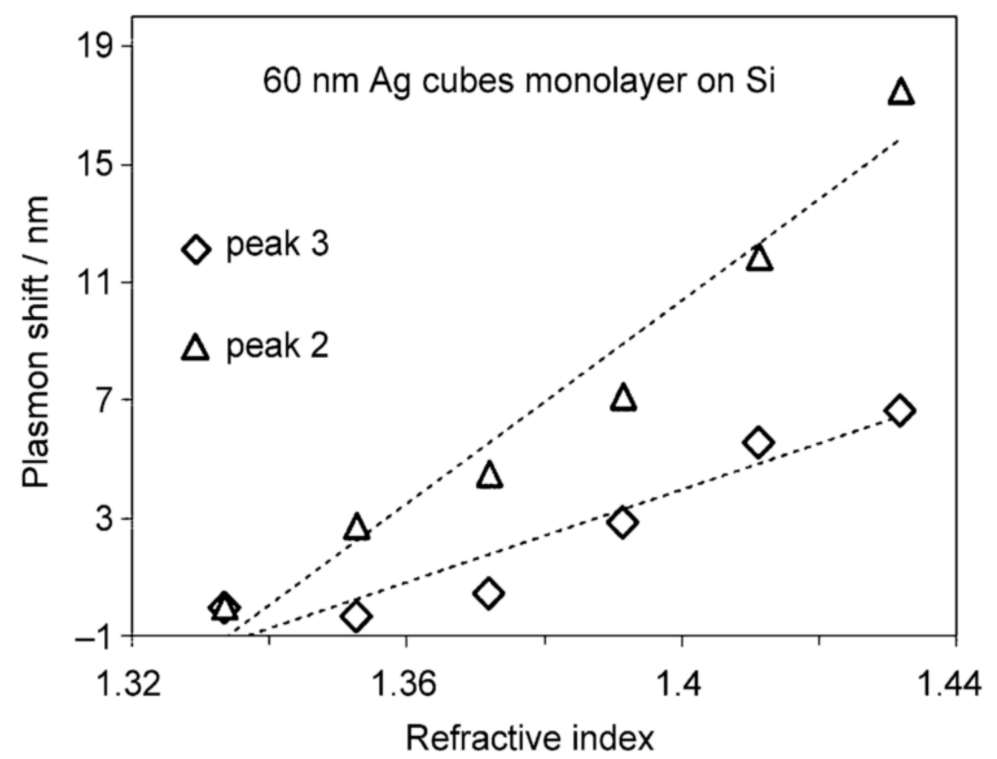

Figure 3.3. Plasmon shifts for dipolar (peak 3) and quadrupolar (peak 2) resonances for a monolayer of silver nanocubes deposited on a thin Si film.

The spectral separation between the D and Q modes can be adjusted if the thickness of the silicon film is varied, resulting in a different refractive index of the substrate. Under certain conditions, this could lead to Fano interference between the dipolar and quadrupolar modes, ${ }^{3}$ which in turn will increase the RIS of the nanoparticles even more. Measurement of 
this kind might need to be performed in a reflection mode as increasing the thickness of such a film will lower its transparency.

In conclusion, we demonstrated that the RIS for a monolayer of silver nanocubes can be as high as that for a single nanoparticle when an appropriate substrate is used. In that respect, silicon films of varying thickness are very useful to possibly induce Fano interference.

\subsection{Experimental}

Silver nitrate $\left(\mathrm{AgNO}_{3}, 99+\%\right)$, polyvinylpyrrolidone (PVP, Mw 55,000), anhydrous 99.8\% ethylene glycol (EG), sodium sulfide, and chloroform were purchased from SigmaAldrich and used as obtained. Ethanol (95\%) was from Commercial Alcohols and used without further purification.

Silver nanocubes (NC) with an edge length of 60nm were synthesized according to the procedure described in the literature. ${ }^{13}$ Typically, a $100 \mathrm{~mL}$ round bottom flask containing $35 \mathrm{~mL}$ of EG and a clean stir bar were placed in an oil bath set to $150^{\circ} \mathrm{C}$ and allowed to heat for one hour. Using a glass syringe, $5 \mathrm{~mL}$ of EG containing $0.4 \mathrm{~g}$ of dissolved PVP was injected. Five minutes later, using a micropipette, 400mL of 3mM sodium sulfide dissolved in EG was added. After an additional $5 \mathrm{~min}, 2.5 \mathrm{~mL}$ of $282 \mathrm{mM}$ silver nitrate dissolved in EG was injected slowly using a glass syringe. Upon addition of the silver nitrate, the solution immediately turned black and slowly became a transparent yellow, then changed to an ochre colour while some plating in the flask occurred. The reaction was monitored by periodically taking small aliquots out of the reaction flask using a pasture pipette and dispersing it in a cuvette filled with ethanol for UV/Vis spectroscopy. The reaction was quenched by placing the flask in an ice bath when the appearance of a small but distinct peak at 350nm was observed in the spectra. The as-synthesized colloidal nanocubes were purified as previously 
described. ${ }^{9}$ Nanocube substrates were prepared according to the procedure reported previously. ${ }^{9}$

UV/Vis spectra of the colloidal nanocubes in ethanol and of monolayers deposited on the substrates were recorded using a Shimadzu, UV-2450 UV/Vis spectrophotometer. Spectra were analyzed using the GRAMS/AI spectral data-processing software. Spectral deconvolution was performed using an appropriate number of mixed Gaussian-Lorentzian functions and a linear baseline to achieve the best fit to the original trace while minimizing the $x^{2}$ values. To determine the RIS, silver-nanocube samples were dispersed in waterethylene glycol solutions with varying volume percentages, and the extinction spectra were measured. The refractive indices of the mixtures were calculated from the volume percentages of the ingredients according to the Lorentz-Lorenz equation. ${ }^{14}$ The refractive indices of the pure solvents were 1.3334 and 1.4318 for water and EG, respectively. ${ }^{14}$ The plasmon shifts were plotted as a function of the refractive index. The RIS were obtained from the slope of the linear fitting. The figures of merit (FOM) were calculated as ratios of RIS to the FWHM for the peaks obtained from the peak-fit- ting procedure.

The topography of the NC monolayer, transferred onto glass substrates at different surface pressures, was obtained using an Ntegra (NTMDT, Russia) atomic force microscope in semi-contact mode in air at $23^{\circ} \mathrm{C}$ with $512 \times 512$ points per image. A $100 \times 100 \mu \mathrm{m}^{2}$ scanner (Ntegra) and cantilevers with rotated monolithic silicon tips (125 $\mu \mathrm{m}$-long, $40 \mathrm{Nm}^{-1}$ spring constant Tap 300Al, resonance frequency $315 \mathrm{kHz}$, Budget Sensors) were used for all topographic measurements. The typical scan rate was $0.5 \mathrm{~Hz}$. AFM images were further processed by the Nova image processing software.

\subsection{References}

1 J. M. McMahon, Y. Wang, L. J. Sherry, R. P. Van Duyne, L. D. Marks, S. K. Gray, G. 
C. Schatz, J. Phys. Chem. C 2009, 113, 2731-2735.

2 L. J. Sherry, S-H. Chang, G. C. Schatz, R. P. Van Duyne, B. J. Wiley, Y. Xia, Nano lett. 2005, 5, 2034-2038.

3 S. Zhang, K. Bao, N. J. Halas, H. Xu, P. Nordlander, Nano lett. 2011, 11, 1657-1663.

4 Y. H. Lee, H. Chen, Q.-H. Xu, J. Wang, J. Phys. Chem. C 2011, 115, 7997- 8004.

$5 \quad$ K. M. Mayer, J. H. Hafner, Chem. Rev. 2011, 111, 3828-3857.

6 E. Ringe, J. M.McMahon, K. Sohn, C. Cobley, Y. Xia, J. Huang, G. C. Schatz, L. D. Marks, R. P. Van Duyne, J. Phys. Chem. C 2010, 114, 12511- 12516.

$7 \quad$ W. J. Galush, S.A Shelby, M. J. Mulvihill, A. Tao, P. Yang, J. T. Groves, Nano lett. 2009, 9, 2077-2082.

8 J. N. Anker, W. P. Hall, O. Lyanders, N. C. Shah, J. Zhao, R. P. Van Duyne, Nat. Mater. 2008, 7, 442-453.

$9 \quad$ N. Ahamad, A. Ianoul, J. Phys. Chem. C 2011, 115, 3587-3594.

10 S. Adachi, Phys. Rev. B 1988, 38, 12966-12976.

11 A. R. Siekkinen, J. M. McLellan, J. Chen, Y. Xia, Chem. Phys. Lett. 2006, 432, 491496.

12 F. Zhou, Z-Y. Li, Y. Liu, Y. Xia, J. Phys. Chem. C 2008, 112, 20233-20240.

13 M. A. Mahmoud, M. A. El-Sayed, Nano Lett. 2011, 11, 946-953.

14 H. Chen, X. Kou, Z. Yang, W. Ni, J. Wang, Langmuir 2008, 24, 5233- 5237. 


\section{Chapter 4}

Optimizing Refractive Index Sensitivity of Supported Silver Nanocube Monolayers

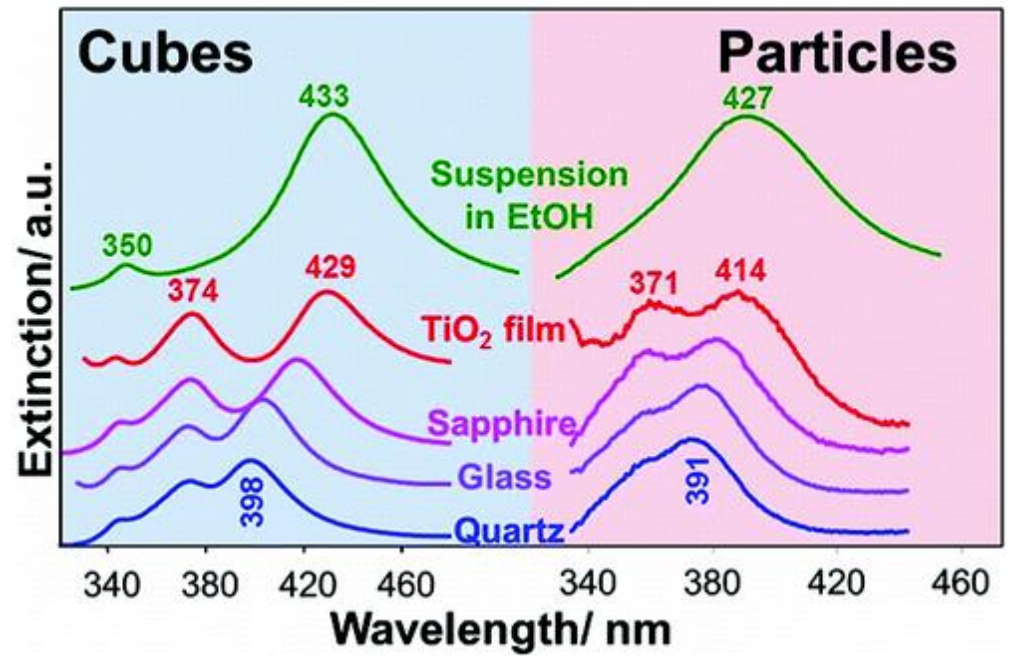


Modified from original manuscript published as:

Ahamad, N., Bottomley, A. and Ianoul, A., Optimizing Refractive Index Sensitivity of Supported Silver Nanocube Monolayers. J. Phys. Chem. C 2012, 116, 185-192.

doi:10.1021/jp207682s

Department of Chemistry, Carleton University, 1125 Colonel By Drive, Ottawa, Ontario, Canada, K1S 5B6

\subsection{Abstract}

The refractive index (RI) sensitivity of extinction spectra was compared experimentally for silver nanocubes in solution and in supported monolayers prepared by the Langmuir technique. The size of the nanocubes, RI of supporting dielectric substrate, and monolayer surface pressure were used as variables in refractive index sensing optimization. The dipolar plasmon modes of the colloidal nanocubes were found to have the highest refractive index sensitivites (RIS) values of 176,361 , and $480 \mathrm{~nm} / \mathrm{RI}$ units for 40 , 80 , and 130nm cubes, respectively. The largest figure of merit (FOM) of 4.55 was measured for a quadrupolar mode of $130 \mathrm{~nm}$ nanocubes. When compared to suspensions, the RIS of supported nanocubes were reduced by $\sim 50 \%$ and decreased with increasing monolayer surface pressure. The RIS of 40nm cube monolayers appeared to be sensitive to the substrate RI due to the RI-dependent plasmon mode hybridization, resulting in dipolar and quadrupolar modes. The intensity of this quadrupolar peak was found to increase with the angle of incident light. This work shows that the use of high refractive index dielectric substrates, a passive molecular spacer, and large angles of incidence can improve the detection of plasmonic response by supported nanocube monolayers.

\subsection{Introduction}

Noble metal nanostructures have been of great interest due to their unique optical properties useful in numerous applications. Recently, significant progress has been made in 
the development of nanoparticle fabrication methods ${ }^{1}$ and theory, ${ }^{2}$ leading to the discovery of novel phenomena. Giant enhancement of the local electric field near the surface of plasmonic nanoparticles due to excitation of their localized surface plasmon resonances (LSPR) has led to an increase in the optical response from molecules in the vicinity of such nanoparticles, resulting in surface-enhanced Raman, ${ }^{3,4}$ fluorescence, ${ }^{5-8} \operatorname{IR}^{9}{ }^{9}$ and secondharmonic generation ${ }^{10}$ phenomena. The localized electric field is also very sensitive to refractive index change in the environment, making LSPR an excellent sensing platform. ${ }^{11-13}$ In addition, interacting plasmonic nanoparticles have recently been shown to possess some novel properties, such as collective plasmonic modes, ${ }^{14,15}$ Fano resonances, ${ }^{16}$ etc., leading to improvement of sensing devices, ${ }^{11,16}$ fabrication of nanoantennas, ${ }^{17,18}$ and nanorulers. ${ }^{19}$

Size, shape, material, and dielectric environment control the plasmonic signature of metal nanoparticles. ${ }^{20-25}$ As the nanoparticles become larger, in addition to a single dipolar LSPR mode, higher order plasmonic modes appear., ${ }^{1,26,27}$ It is well recognized that interactions with a support (dielectric or conducting) as well as interparticle separation and local order are crucial factors in controlling the plasmonic signature. LSPR-induced charges in a dielectric substrate disturb the nanoparticles' electric field, leading to splitting of a single dipolar plasmonic mode into two. ${ }^{28-32}$ For spherical particles these two modes correspond to dipolar oscillations with orthogonal polarizations. ${ }^{28}$ For nanocubes, however, interaction with the substrate leads to plasmon mode hybridization, resulting in two bands, dipolar and quadrupolar, with corresponding charge oscillations located at the opposite sides of the nanocube. ${ }^{29-32}$ The wavelength difference between the two bands was found to be greater than that for the spherical particles, dependent on the size, ${ }^{32}$ edge sharpness, ${ }^{29}$ and separation from the substrate. ${ }^{29,32}$ The bands show strong dependence on the refractive index of the medium. 
Detailed investigations of the plasmon mode hybridization have been performed at the single supported nanoparticle level. ${ }^{29-31}$ Such approach provides certain advantages over the ensemble measurements, due to the increased sensitivity and also narrower plasmonic bands. ${ }^{33}$ However, single-nanoparticle measurements are difficult to perform when the size is smaller than 20nm. ${ }^{13}$ Also, for routine and consistent measurements it is important to develop substrates that can be easily and reproducibly fabricated. ${ }^{12,13}$ Additionally, certain plasmonic phenomena can be only observed for interacting nanoparticles. Therefore, it is important to investigate the unique properties, particularly the refractive index sensitivity of silver nanocubes in monolayers where the degree of interparticle coupling can be controlled. To the best of our knowledge, no detailed studies of this kind have been performed. Although the single nanoparticles usually have narrower bands, if the sample has high monodispersity in particle size the plasmonic bandwidth for the ensemble can be narrow as well. In the present work we systematically investigated the refractive index sensitivity of monodisperse silver nanocubes, both in suspension and in monolayers transferred onto a solid support, by the Langmuir technique as a function of nanocube size and spacing as well as dielectric properties of a substrate.

\subsection{Materials and Methods}

Chemicals: Silver nitrate $\left(\mathrm{AgNO}_{3}, 99+\%\right)$, polyvinylpyrrolidone (PVP, $\mathrm{M}_{\mathrm{w}} \approx 55$ 000), anhydrous 99.8\% ethylene glycol (EG), sodium sulfide, and chloroform were purchased from Sigma-Aldrich and used as obtained. Hydrochloric acid ( $\mathrm{HCl}, 38 \%)$ and ethanol (95\%) were obtained from Anachemia and Commercial Alcohols, respectively, and used without further purification. 1,2-Dipalmitoyl-sn-glycero-3-phosphoethanolamine- $N$ (lissamine rhodamine B sulfonyl) (ammonium salt, DPPE-RhB) was purchased from Avanti Polar Lipids as a powder and used as provided. 
Preparation of Nanocubes: Silver nanocubes (NC) with an edge length of 40nm were synthesized according to the procedure described in the literature. ${ }^{34}$ Typically, a $100 \mathrm{~mL}$ round-bottom flask containing 35mL of EG and a clean stir bar was placed in an oil bath set to $150^{\circ} \mathrm{C}$ and allowed to heat for $1 \mathrm{~h}$. Using a glass syringe $5 \mathrm{~mL}$ of EG containing $0.4 \mathrm{~g}$ of dissolved PVP was injected. Five minutes later using a micropipet $400 \mu \mathrm{L}$ of $3 \mathrm{mM}$ sodium sulfide dissolved in EG was added. After an additional $5 \mathrm{~min} 2.5 \mathrm{~mL}$ of $282 \mathrm{mM}$ silver nitrate dissolved in EG was injected slowly using a glass syringe. Upon addition of the silver nitrate the solution immediately turned black, slowly became a transparent yellow, and then changed to an ochre colour while some plating in the flask occurred. The reaction was monitored by periodically taking small aliquots out of the reaction flask using a pasture pipet and dispersing it in a cuvette filled with ethanol for UV-vis spectroscopy. The reaction was quenched by placing the flask in an ice bath when the appearance of a small but distinct peak at 350nm was observed in the spectra.

Cubes with edge lengths of 80 and 130nm: a colloidal solution of silver NC was synthesized by a modified polyol process developed by Xia et al. ${ }^{35}$ In a typical synthesis, $5 \mathrm{~mL}$ of EG was placed in a $100 \mathrm{~mL}$ triple-necked round-bottom flask and heated at $140^{\circ} \mathrm{C}$ for 1h under stirring with a Teflon-coated magnetic stirring bar using a temperature-controlled silicon oil bath. While EG was heated, EG solutions containing $\mathrm{AgNO}_{3}(94 \mathrm{mM})$ and PVP (147mM) were prepared. One milliliter of HCl solution in EG (3mM), prepared 30min prior to completion of EG heating time, was added quickly. Ten minutes after injection of $\mathrm{HCl}$ 3mL of both $\mathrm{AgNO}_{3}$ and PVP solutions were added simultaneously in the hot reaction mixture at a rate of $\sim 750 \mathrm{~mL} / \mathrm{min}$ using glass syringes. The resulting solution was allowed to heat for $26 \mathrm{~h}$ under reflux conditions. The final product was cooled to room temperature and subjected to further purification. 
Purification of Colloidal Silver Nanocubes: The as synthesized colloidal nanocubes contained unreacted EG, an excess of PVP, and nanorods as a byproduct. EG and PVP were removed by diluting the colloidal solution with ethanol (1:1 by volume) followed by centrifugation at $12000 \mathrm{rpm}$. The precipitate was collected and redispersed in ethanol by sonication. This process was repeated 30 times for complete removal of EG and PVP. Silver nanorods were separated from the nanocubes by filtering the prewashed NC in ethanol using PTFE syringe filters (pore size 0.45 and $0.22 \mu \mathrm{m}$ ). The purified silver NC solution was further subjected to washing with chloroform by centrifugation (at least 3 times) prior to LB film preparation. The final volume of the nanocube solution was $4 \mathrm{~mL}$.

Preparation of LB Monolayers: Nanocube substrates were prepared according to the procedure reported previously. ${ }^{36}$ Briefly, a NIMA 311D trough, filled with Milli-Q water (18.2 $\mathrm{m} \Omega \mathrm{cm})$, was used to prepare the Langmuir monolayers of different NC samples. In order to form monolayers, an appropriate amount of each sample was deposited onto the water surface using a microsyringe. Each monolayer was left for 20min to allow chloroform evaporation. The surface pressure of the monolayer was measured with a paper Wilhelmy plate. Before transferring the monolayer onto solid supports several isotherm cycles were performed to anneal the films to the maximum pressure of $20-25 \mathrm{mN} / \mathrm{m}$. Monolayers of pure nanocubes and nanocube/lipid DPPE-RhB mixtures were transferred onto substrates cleaned with aqua regia and subsequently with a mixture of chloroform and methanol at various surface pressures by vertical upward dipping at $2 \mathrm{~mm} / \mathrm{min}$ at room temperature (usually 25$\left.30^{\circ} \mathrm{C}\right)$.

UV-Vis Measurement: UV-vis spectra of the colloidal nanocubes in ethanol and of monolayers deposited on the substrates were recorded using a Shimadzu UV-2450 UV-vis spectrophotometer. Spectra were analyzed using GRAMS/AI spectral data processing 
software. Spectral deconvolution was performed using an appropriate number (for the spectral region of interest we used five components) of mixed Gaussian-Lorentzian functions as well as a linear baseline to achieve the best fit to the original trace while minimizing $\chi^{2}$ values. To determine the refractive index sensitivities (RIS), silver nanocube samples (colloidal or deposited on slides) were dispersed in water-ethylene glycol mixture solutions (slides with supported nanocubes were immersed into solvent mixtures) with varying volume percentages and the extinction spectra measured. The refractive indices of the mixtures were calculated from the volume percentages of the ingredients according to the Lorentz-Lorenz equation. ${ }^{37}$ The refractive indices of the pure solvents were 1.3334 and 1.4318 for water and EG, respectively. ${ }^{37}$ The plasmon shifts were plotted as a function of the refractive index. The refractive index sensitivities were obtained from the slope of the linear fitting. The figures of merit (FOM) were calculated as ratios of RIS to the full width at halfmaximum (fwhm) for the peaks obtained from the peak-fitting procedure.

Topography Measurements: The topography of the NC monolayer, transferred onto glass substrates at different surface pressures, was obtained using an Ntegra (NTMDT, Russia) atomic force microscope in semi contact mode in air at $23^{\circ} \mathrm{C}$ with $512 \times 512$ points per image. A $100 \times 100 \mu \mathrm{m}^{2}$ scanner (Ntegra) and cantilevers with rotated monolithic silicon tips $(125 \mu \mathrm{m}$ long, $40 \mathrm{~N} / \mathrm{m}$ spring constant Tap 300Al, resonance frequency $315 \mathrm{kHz}$, Budget Sensors) were used for all topographic measurements. The typical scan rate was $0.5 \mathrm{~Hz}$. AFM images were further processed by Nova image processing software.

\subsection{Results and Discussion}

Effect of Nanocube Size on the Refractive Index Sensitivity: Three batches of silver nanocubes were prepared is this work with edge lengths of 40, 80, and 130nm. Figure 4.1 shows representative AFM images of monolayers deposited by the Langmuir technique 
at low surface pressures as well as histograms of the height distribution in the samples. After purification by numerous rounds of centrifugation and filtration, the resulting nanocube samples were of high monodispersity, without nanorod impurities and with a size variation of less than $10 \%$. Inspection of the nanocube samples using SEM demonstrated that cubes had well- defined sharp edges. As a result of such high monodispersity UV-vis spectra show characteristic and narrow plasmonic extinction bands (Figure 4.2).
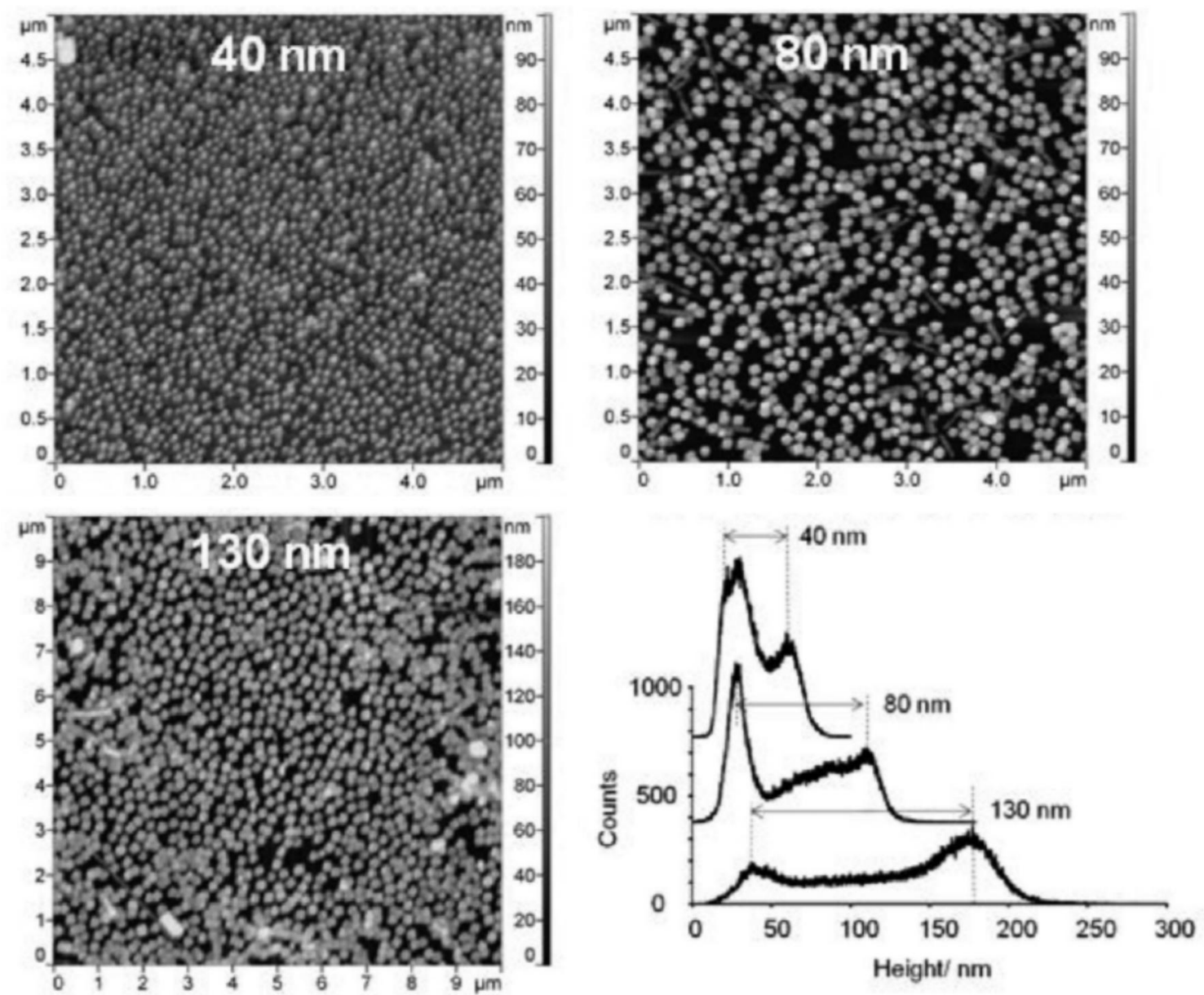

Figure 4.1. Atomic force microscopy of supported nanocube substrate prepared by Langmuir monolayer technique at low $(0.1-1 \mathrm{mN} / \mathrm{m}, 15 \mathrm{mN} / \mathrm{m}$ for $130 \mathrm{~nm}$ cubes) surface pressure. Histograms show height distribution in the samples.

The extinction spectrum for the 40nm nanocube suspension shows two sharp peaks (Figure 4.2A): a dipolar mode at $428 \mathrm{~nm}$ and a peak at $350 \mathrm{~nm}$ that is often assigned as an octupolar plasmonic mode. ${ }^{38}$ As the size of the nanocubes increases, new bands appear and 
the dipolar peak shifts to lower energies. It has been found that when the size of silver nanocubes exceeds 40-50nm a quadrupolar peak appears. ${ }^{1,39}$ Therefore, for $80 \mathrm{~nm}$ cubes in addition to a dipolar mode at 530nm we observed a quadrupolar mode at $\sim 450 \mathrm{~nm}$ and a peak at $400 \mathrm{~nm}$ which is often assigned to a quadrupolar mode ${ }^{38}$ but also to mixed dipole/ quadrupole plasmonic mode ${ }^{27}$ and a peak at 350nm (Figure 4.2B). For a 130nm cube sample the dipolar peak is found at $\sim 680 \mathrm{~nm}$, the first quadrupolar at 530nm, the second quadrupolar (hybrid dipolar/quadrupolar) at $\sim 404 \mathrm{~nm}$, and a peak at 350nm (Figure 4.2C).

Peak fitting of the spectra was performed using mixed Gaussian-Lorentzian functions. The peaks half widths were found to increase with the nanocubes size. For example, the fwhm of the dipolar peak increases from 55 to 235 to 330nm for 40, 80, and 130nm nanocubes, respectively (Table 4.1). The same trend was observed for the quadrupolar peaks. 


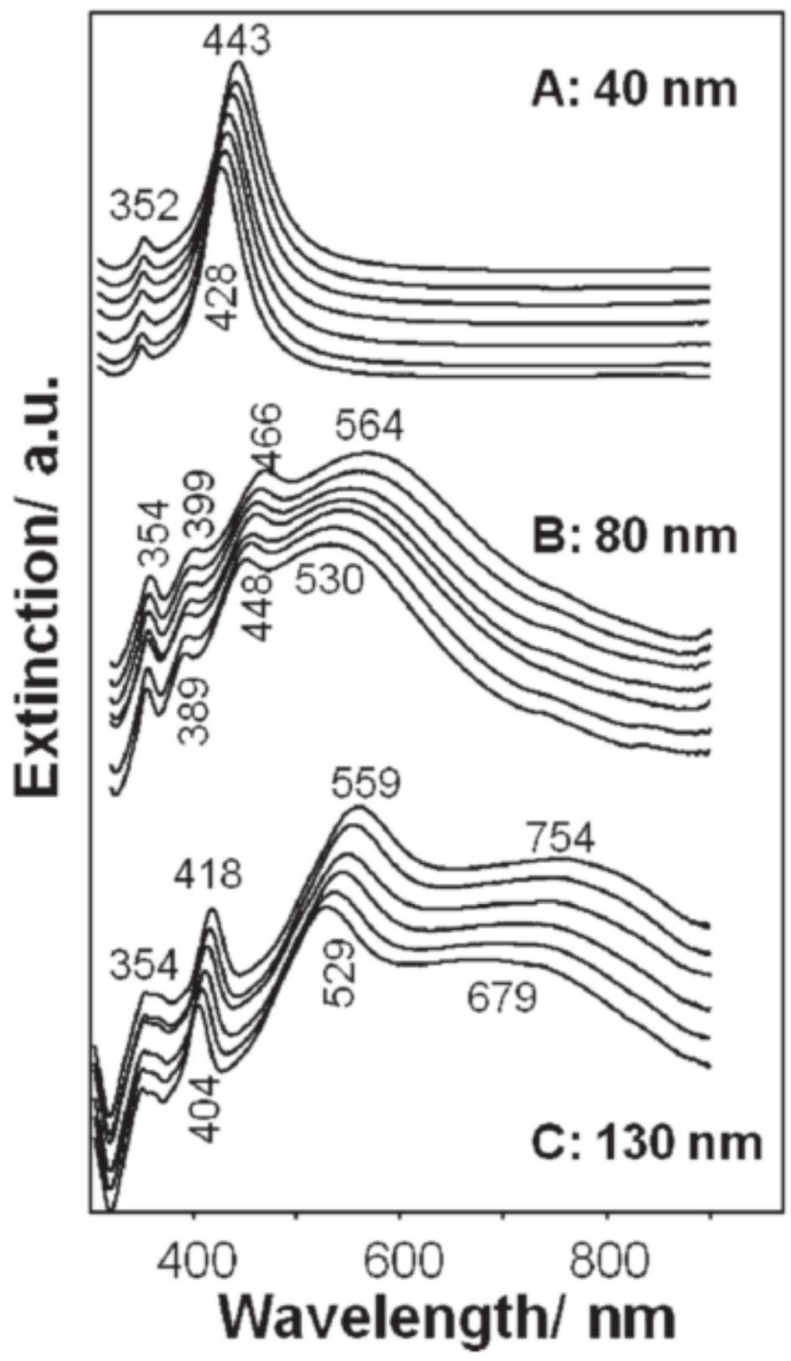

Figure 4.2. Extinction spectra of colloidal suspensions in different water/ethylene glycol solutions for (A) 40, (B) 80, and (C) 130nm nanocubes. Spectra were measured in water (lowest spectra in series), followed by water:EG 4:1, ethanol (40 and 80nm only), water:EG 3:2, 2:3, 1:4, and pure EG.

Extinction spectra of silver nanocubes in suspension were further measured in different solvent mixtures with increasing refractive index to probe the refractive index sensitivity. The Figure 4.2 series consists of extinction spectra obtained in water (the lowest spectra in the series) followed by water:EG 4:1 mixture, ethanol, water:EG 3:2, 2:3, and 1:4 mixtures, and pure EG. A plot showing the plasmonic shifts versus their corresponding refractive indices is shown in Figure 4.3. As expected, the positions of the plasmonic bands red shift as the refractive index of the solvent increases. The experimentally obtained RIS are 
listed in Table 4.1. The highest RIS was determined for the dipolar peak of $130 \mathrm{~nm}$ nanocubes, 480nm/RIU. Figures of merit, measured as a ratio of RIS to the peak half width, on the other hand, decreased with size (Table 4.1). The highest figure of merit of 4.55 was determined for the peak attributed to the dipolar/quadrupolar mixed plasmonic mode of the 130nm cubes.

Table 4.1. Refractive Index Sensitivities of Silver Nanocubes in Suspension

\begin{tabular}{ccccc} 
size (nm) & $\begin{array}{c}\text { plasmon peak } \\
\text { position }(\lambda / \mathrm{nm})\end{array}$ & $\begin{array}{c}\text { full width at half } \\
\text { maximum }(\mathrm{nm})\end{array}$ & $\begin{array}{c}\text { refractive index } \\
\text { sensitivity (nm/RIU) }\end{array}$ & figure of merit \\
\hline \multirow{2}{*}{40} & 428 & 55 & $176 \pm 14$ & 3.3 \\
& 350 & 15 & $29 \pm 1.6$ & 1.88 \\
\multirow{2}{*}{80} & 530 & 235 & $361 \pm 50$ & 2.43 \\
& 448 & 65 & $158 \pm 42$ & 1.54 \\
& 389 & 40 & $74 \pm 26$ & 1.83 \\
& 350 & 22 & $20 \pm 9$ & 0.9 \\
& 679 & 330 & $480 \pm 74$ & 1.46 \\
& 529 & 138 & $304 \pm 29$ & 2.2 \\
& 404 & 27 & $125 \pm 14$ & $\mathbf{4 . 5 5}$
\end{tabular}

Data presented here are consistent with recently published results. ${ }^{38}$ For example, the RIS of $336 \pm 15 \mathrm{~nm} / \mathrm{RIU}$ measured for the dipolar band of $84 \pm 6 \mathrm{~nm}$ Ag nanocubes ${ }^{38}$ is very close to $360 \pm 50 \mathrm{~nm} / \mathrm{RIU}$ obtained in this work for $80 \pm 5 \mathrm{~nm}$ cubes. The RIS for other plasmon modes are also similar. However, the figures of merit do not match very well, likely due to variations in the edge and corner sharpness of the nanocubes as well as different peakfitting procedures used. 

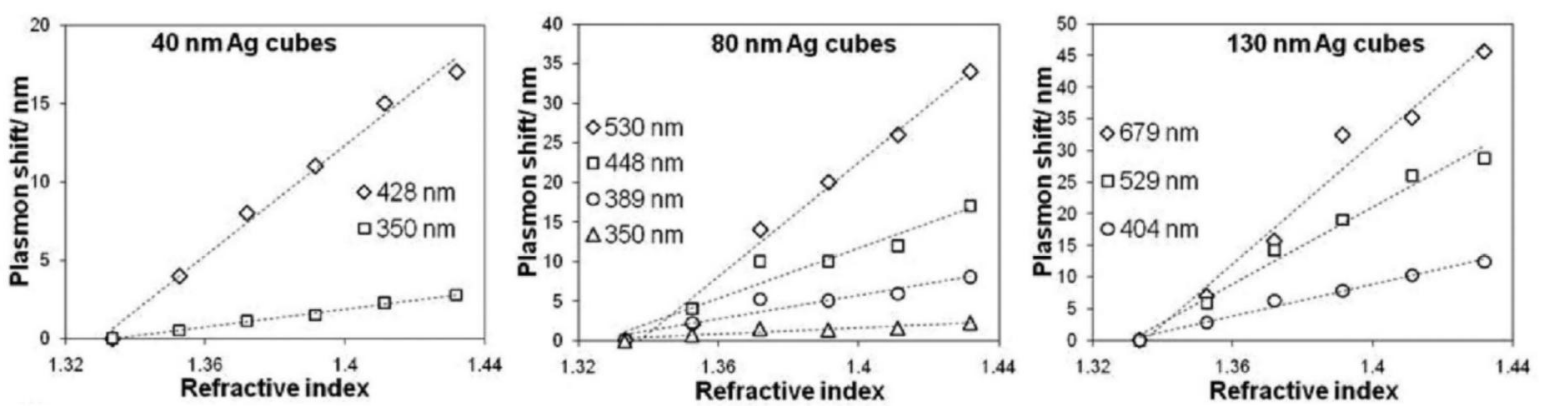

Figure 4.3. Plasmonic shifts as a function of the refractive index for silver nanocube suspensions.

\section{Refractive Index Sensing by Supported vs Suspended Nanocubes: Often} applications require ensembles of nanoparticles to be deposited on a solid support for better reproducibility and control. ${ }^{12,13}$ Interaction with a solid support, however, is known to alter the plasmonic optical properties of metal nanoparticles, such as refractive index sensitivity. ${ }^{30}$ To test the effect of solid dielectric support on the refractive index sensing by nanocube ensembles of different size we used Langmuir monolayer technique. The technique enables facile and reproducible fabrication of substrates with controlled interparticle separation and density that is dependent on the surface pressure at which the monolayer is transferred. ${ }^{36}$ We first looked at the substrates prepared at low surface pressure to minimize the interparticle interactions and to facilitate the comparison with nanocubes in suspension. At such low pressure the dipole-dipole near-field coupling between adjacent nanocubes is weak, as can be monitored using a low-energy broad band in the spectra (for 130nm cubes sample such conditions maintained even at $15 \mathrm{mN} / \mathrm{m})$.

Figure 4.4 shows extinction spectra of supported nanocube substrates for three sizes in air (lowest, separate spectra in the series) and immersed in liquids with an increasing refractive index. When deposited on a solid support the spectra change considerably compared to those in colloidal suspension. For the largest 130nm nanocubes studied the dipolar peak shifts from 680 to $477 \mathrm{~nm}$, the first quadrupolar shifts from 529 to $435 \mathrm{~nm}$, the 
second quadrupolar/mixed from 400 to $369 \mathrm{~nm}$, and a peak at 350 to $340 \mathrm{~nm}$. The same trend is observed for the 80nm sample, with all four bands blue shifting.

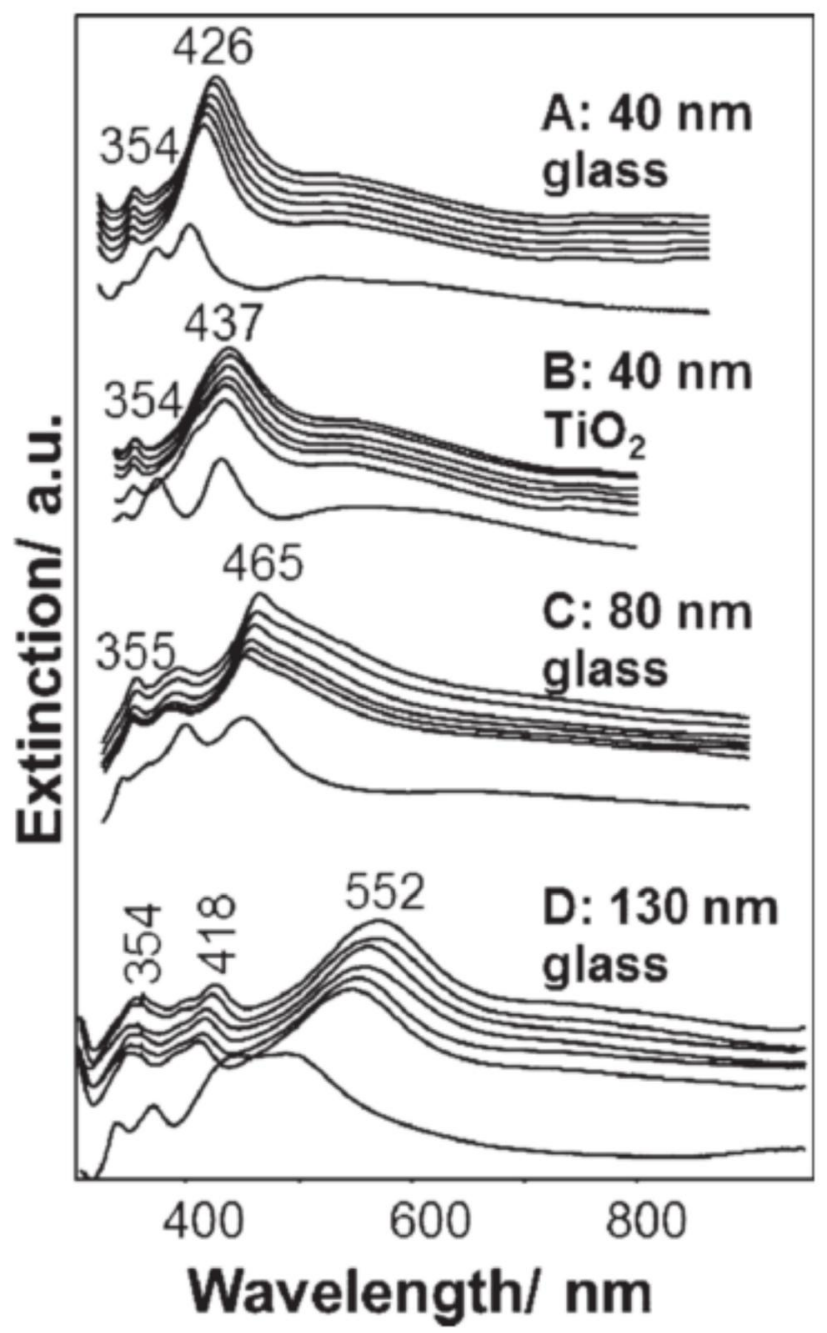

Figure 4.4. Extinction spectra of supported nanocube monolayers in air (lowest spectra in series) and immersed in different water/ethylene glycol solutions for (A) $40 \mathrm{~nm}$ on a glass substrate, (B) $40 \mathrm{~nm}$ on a glass substrates coated with $5 \mathrm{~nm} \mathrm{TiO} 2$ film, (C) $80 \mathrm{~nm}$, and (D) $130 \mathrm{~nm}$ nanocubes. Spectra were measured in water (first spectra in series), followed by water:EG 4:1, water:EG 3:2, 2:3, 1:4, and pure EG.

For the 40nm cubes, however, the substrate induced a more dramatic spectral change. For the dipolar peak at 428nm plasmon mode hybridization takes place resulting in two peaks: dipolar at $\sim 400 \mathrm{~nm}$ (fwhm $\approx 35 \mathrm{~nm}$ ) and quadrupolar at $\sim 374 \mathrm{~nm}$ ( fwhm $\approx 20 \mathrm{~nm}$ ). Unlike larger nanocubes, the quadrupolar mode cannot be excited in $40 \mathrm{~nm}$ cubes in a suspension. ${ }^{1,39}$ However, when the substrate is present plasmon induces charge oscillations in 
the substrate which act to disturb the electric field in the nanocube. ${ }^{30,31}$ As a result, two new modes can be excited: a lower energy dipolar mode with charge oscillating at the side of the nanocube near the substrate and a higher energy quadrupolar mode with corresponding charge oscillations at the nanocube side away from the substrate. The wavelength difference between the two peaks increases with the substrate refractive index (will be discussed later).

Table 4.2. Refractive Index Sensitivities of Supported Silver Nanocube Monolayers on Glass at Low ${ }^{a}$ Surface Pressure

\begin{tabular}{ccccc} 
size (nm) & $\begin{array}{c}\text { plasmon peak } \\
\text { position }(\lambda / \mathrm{nm})\end{array}$ & $\begin{array}{c}\text { full width at half } \\
\text { maximum }(\mathrm{nm})\end{array}$ & $\begin{array}{c}\text { refractive index } \\
\text { sensitivity }(\mathrm{nm} / \mathrm{RIU})\end{array}$ & figure of merit \\
\hline 40 & 420 & 44 & $93 \pm 11$ & 2.11 \\
$40, \mathrm{TiO}_{2}$ & 352 & 13 & $31 \pm 2$ & $\mathbf{2 . 4}$ \\
& 420 & 54 & $57 \pm 5$ & 1.05 \\
& 400 & 27 & $90 \pm 5$ & 3.3 \\
80 & 350 & 10 & $29 \pm 1.1$ & 2.9 \\
& 480 & 159 & $73 \pm 27$ & 0.46 \\
& 450 & 42 & $89 \pm 20$ & $\mathbf{2 . 1 2}$ \\
& 380 & 46 & $46 \pm 13$ & 1.0 \\
& 350 & 23 & $39 \pm 10$ & n.d. \\
& 700 & n.d. & n.d. & 1.2 \\
& 527 & 128 & $155 \pm 32$ & $\mathbf{2 . 6}$ \\
& 409 & 30 & $77 \pm 11$ & 1.1
\end{tabular}

${ }^{a}$ Surface pressure between 0.1 and $1 \mathrm{mN} / \mathrm{m}$ for 40 and $80 \mathrm{~nm}$ cubes. For $130 \mathrm{~nm}$ nanocubes the surface pressure was $15 \mathrm{mN} / \mathrm{m} .{ }^{b}$ Not determined

The half widths of all bands decrease for supported nanocubes likely due to introduction of a preferred orientation with all the cubes aligned in the same plane. It is possible however that occasional dimer formation broadens the spectra in solutions. The measured fwhm for the dipolar bands are $35 \mathrm{~nm}$ for $40 \mathrm{~nm}$ cubes, $80 \mathrm{~nm}$ for $80 \mathrm{~nm}$ cubes, and $130 \mathrm{~nm}$ for $130 \mathrm{~nm}$ (Table 4.2). This is about one-half the widths measured for the 
suspensions, which is useful for increasing the FOM values.

When the slides with glass-supported nanocube monolayers are immersed into solutions for measuring the refractive index response the spectra undergo significant changes. In all cases red shifting of the plasmonic bands was observed. For the 40nm cubes the hybridized plasmonic mode disappeared and a single band at 415nm emerges when the substrate is immersed in water (Figure 4.4A, lower spectrum). For the 80nm substrate the dipolar band broadens with fwhm increasing to $160 \mathrm{~nm}$ and red shifts from 450 to $\sim 480 \mathrm{~nm}$ and the quadrupolar band remains fairly sharp with $45 \mathrm{~nm}$ fwhm and red shifts from 400 to 450nm. For the biggest nanocubes the dipolar peak broadens to more than $200 \mathrm{~nm}$ fwhm and shifts to $\sim 700 \mathrm{~nm}$ and the quadupolar peak to $530 \mathrm{~nm}$ with fwhm of $\sim 130 \mathrm{~nm}$.
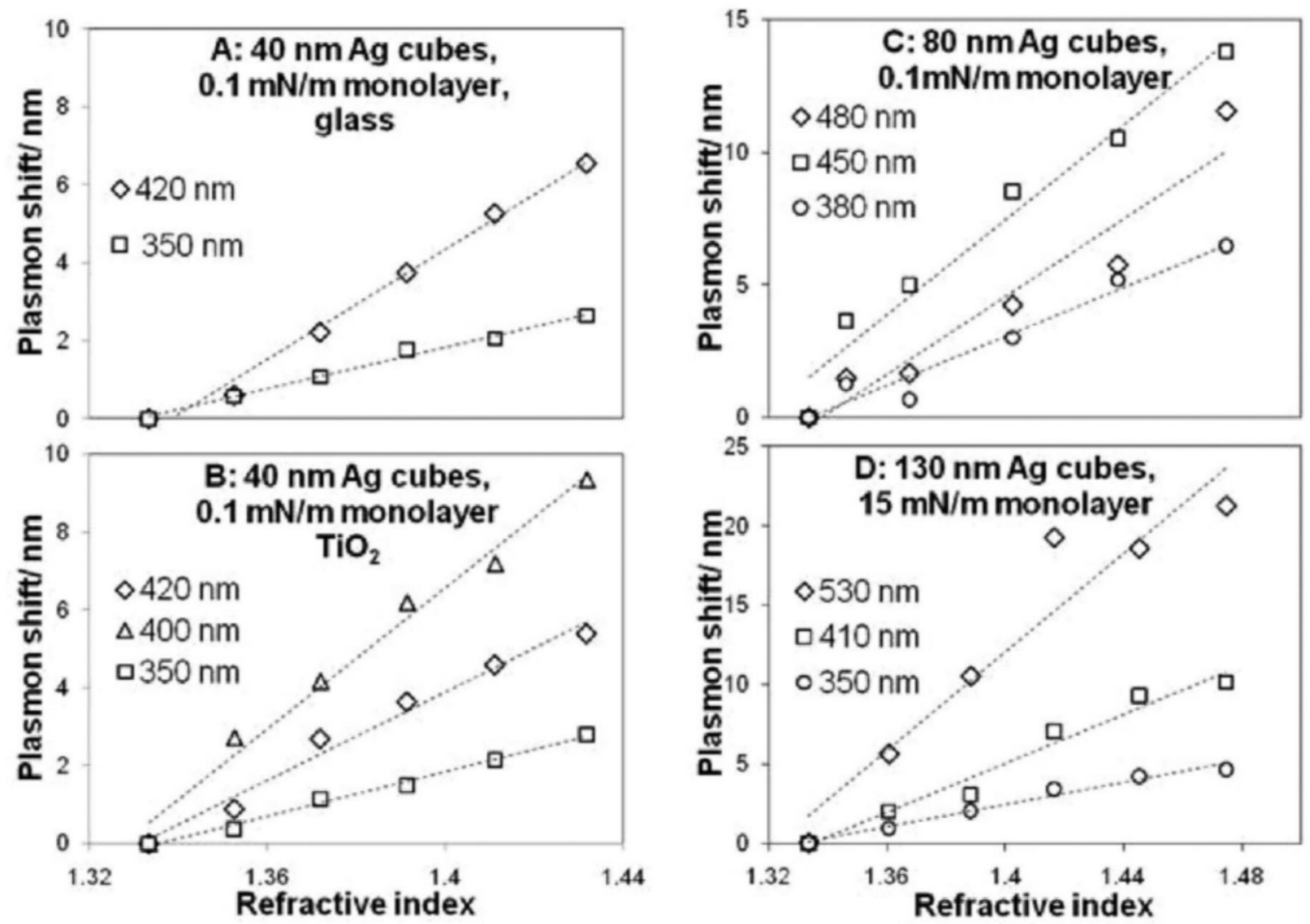

Figure 4.5. Plasmonic shifts as a function of the refractive index for supported silver nanocubes.

When plasmonic shifts are plotted as a function of the refractive index (Figure 4.5), 
refractive index sensitivities as well as FOM can be calculated (Table 4.2). RIS values are considerably smaller for the supported nanocubes than in suspension, particularly for the dipolar modes. The FOM are smaller as well but to a lesser extent than RIS due to the sharper band profiles for the supported nanocubes. The reduced sensitivity of the dipolar mode of supported nanocubes to the solution refractive index change is a direct result of the charge oscillations corresponding to this mode near the surface of the substrate, thus making the mode less sensitive to bulk refractive index change. ${ }^{30,31}$

If the nanocube monolayers are prepared at a higher surface pressure $(>5 \mathrm{mN} / \mathrm{m}$, above the transition from the gas to condensed phase in the nanocube monolayer) the nanocubes aggregate and the dipolar and quadrupolar bands merge due to near-field coupling. ${ }^{36,40-42}$ As a result, it becomes impossible to separate the two modes and the refractive index sensitivity of such supported monolayer drops dramatically (Figure 4.6). At the same time, if a passive spacer is introduced in the monolayer prior to the transfer to prevent the nanocubes from aggregating, the two modes are well separated even at a high surface pressure $^{36}$ and the RIS and FOM remain unchanged up to the $35 \mathrm{mN} / \mathrm{m}$ surface pressure. 

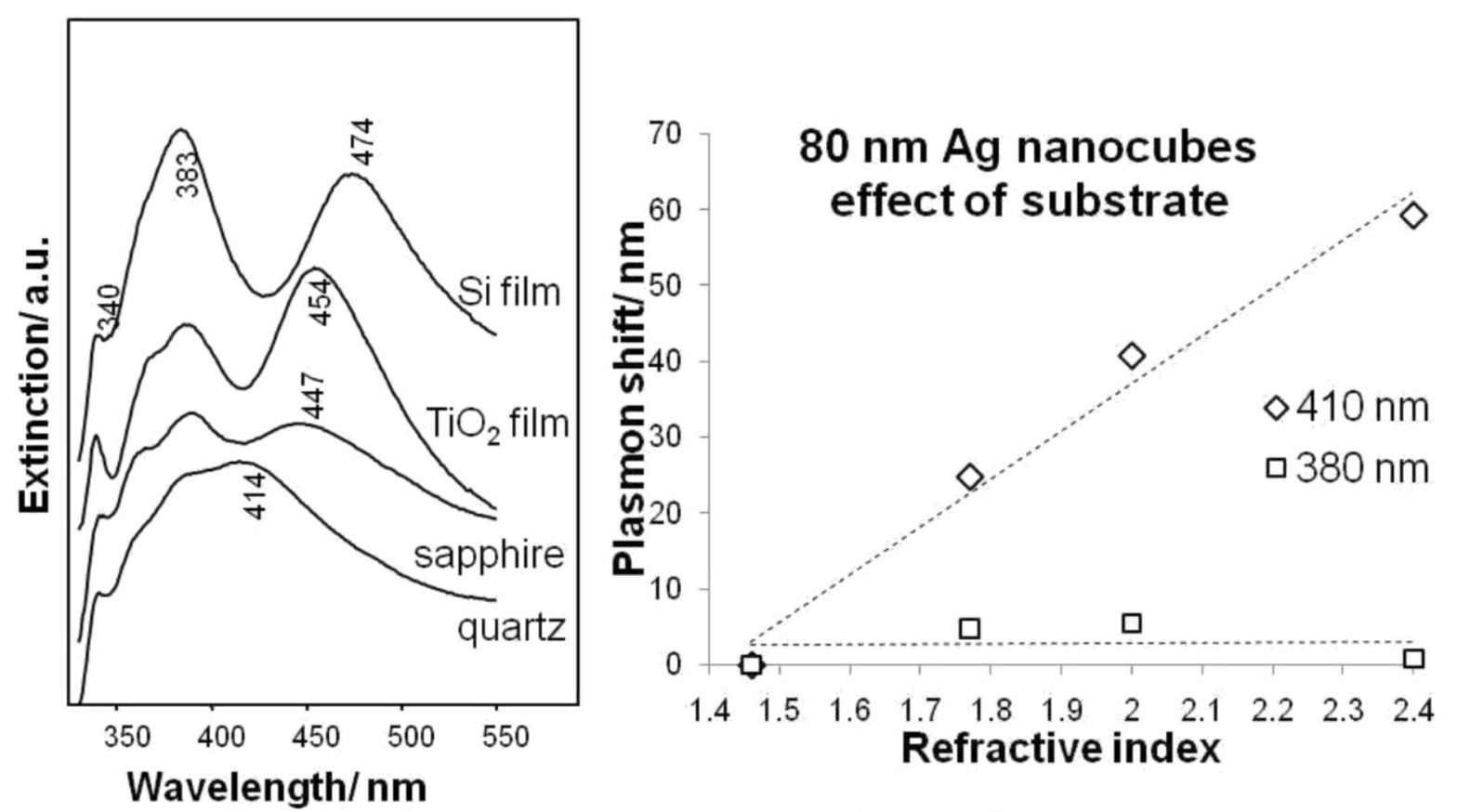

Figure 4.6. Left: UV-vis spectra of supported 80nm nanocubes on different substrates: quartz, sapphire, glass slide coated with $5 \mathrm{~nm} \mathrm{TiO}_{2}$ film, glass slide coated with $5 \mathrm{~nm}$ of silicon. Right: Plasmon shifts plotted vs. the refractive index of a substrate.

It has to be mentioned, however, that the "sharpness" of the spectral peaks decreased after several series of measurements in solutions, likely due to the previously reported smoothening/etching of the nanocube edges. ${ }^{30,43}$ As a result, the RIS and FOM decreased.

It is evident from results presented here that in terms of the figure of merit the quadrupolar mode of supported nanocube monolayers is the most sensitive to the solution refractive index change due to the location of the oscillating charges away from the surface. This confirms previous experimental and theoretical considerations. ${ }^{30,31}$ Here we also observe the disappearance of the quadrupolar mode when the substrate is immersed in liquid, especially for smaller nanocubes. Although some averaging over a large ensemble of particles takes place, the fwhm of the substrates used in the present work is fairly narrow and comparable to that of single-nanocube measurements. For example, the $\sim 20 \mathrm{~nm}$ fwhm measured for the quadrupolar mode of $40 \mathrm{~nm}$ cubes in air (Figure 4.4A) is comparable to that 
of $0.146 \mathrm{eV}^{30}\left(22 \mathrm{~nm}^{11}\right)$ fwhm for a single silver nanocube of comparable size. AFM images also confirm that the particles are fairly monodisperse. The disappearance of the quadrupolar band is therefore not a result of averaging but rather of an insufficiently large difference between the refractive indexes of the support and the solution to induce plasmon mode hybridization. ${ }^{31}$ It is hence desirable to create conditions under which this mode is present even when immersed in solution. Such conditions can be likely obtained when the refractive index difference between the solvent and the substrate is increased. Therefore, we selected several substrates with different refractive indices and prepared monolayers of 40 and $80 \mathrm{~nm}$ nanocubes.

Effect of Substrate on the Refractive Index Sensitivity: Figure 4.7A shows extinction spectra of 40nm silver nanocube monolayers on different substrates with increasing refractive index (spectra 1-4) as well as the colloidal solution spectrum measured in ethanol (6). Instead of a dipolar mode, which is present in solution at 430nm, two new dipolar (D) and quadrupolar (Q) modes appear when supported on a dielectric substrate. The separation between the two bands depends linearly on the refractive index of the substrate (Figure 4.8). However, the dependence of the D band observed at a longer wavelength is more pronounced, as it shifts from $\sim 398 \mathrm{~nm}$ for a quartz substrate $(\mathrm{n}=1.44)$ to $429 \mathrm{~nm}$ for a substrate with $5 \mathrm{~nm} \mathrm{TiO}_{2}$ film on glass, for which the refractive index of $\sim 2.0$ was measured. At the same time the Q mode shifts only by $3 \mathrm{~nm}$. We calculated the sensitivities of the two bands to the substrate refractive index to be 56 and $4.6 \mathrm{~nm} / \mathrm{RIU}$ for the dipole and quadrupole modes, respectively. The corresponding FOM are 1.5 and 0.2 . The two bands are fairly narrow with a fwhm of $\sim 40$ and $20 \mathrm{~nm}$ for $\mathrm{D}$ and Q modes, respectively. 


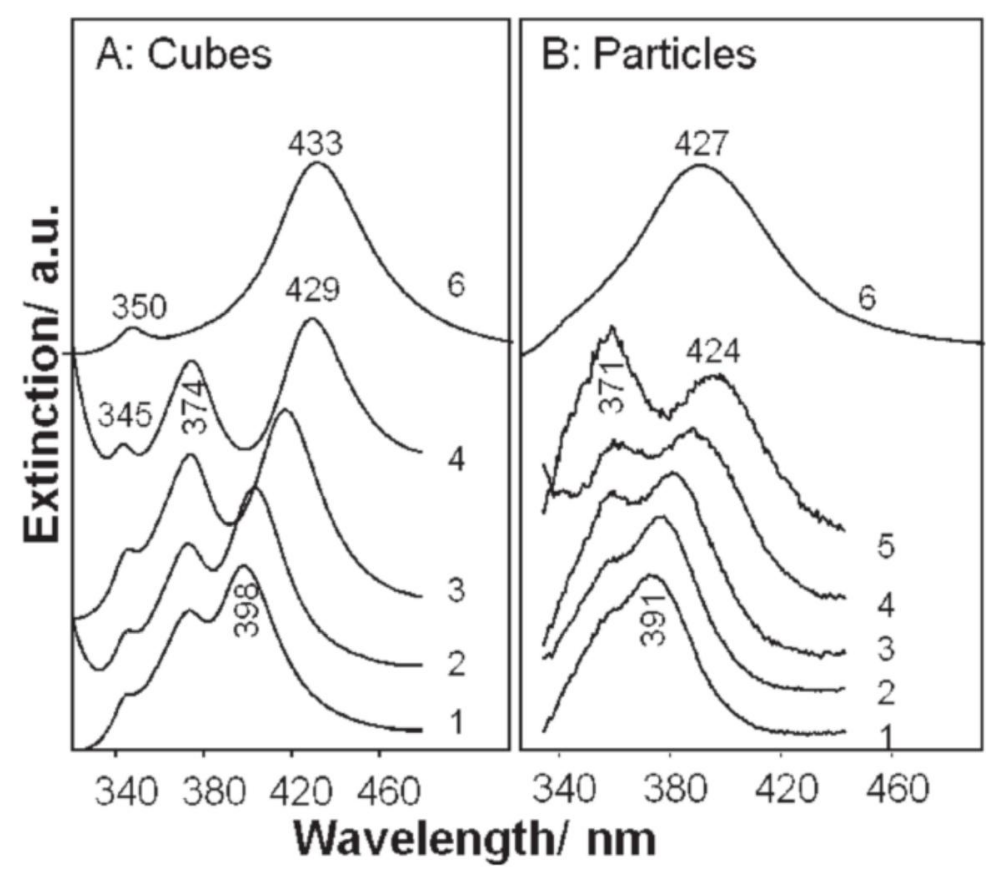

Figure 4.7. UV-vis spectra of supported 40nm nanocubes (A) and irregular-shaped nanoparticles (B) on different substrates: (1) quartz, (2) glass, (3) sapphire, (4) glass slide with coated with $5 \mathrm{~nm} \mathrm{TiO}_{2}$ film, (5) glass slide coated with $5 \mathrm{~nm}$ of silicon, (6) spectra of suspensions in ethanol.

A similar trend was observed for larger nanocubes. For monolayers of 80nm nanocubes prepared on different substrates the dipolar mode gradually shifted from 410 to 436 to 452 to $471 \mathrm{~nm}$ for quartz, sapphire, $\mathrm{TiO}_{2}$, and silicon films respectively giving a refractive index sensitivity of $62 \mathrm{RIU} / \mathrm{nm}$. At the same time, positions of the quadrupolar and octupolar bands remained fairly constant (Figure 4.6). 


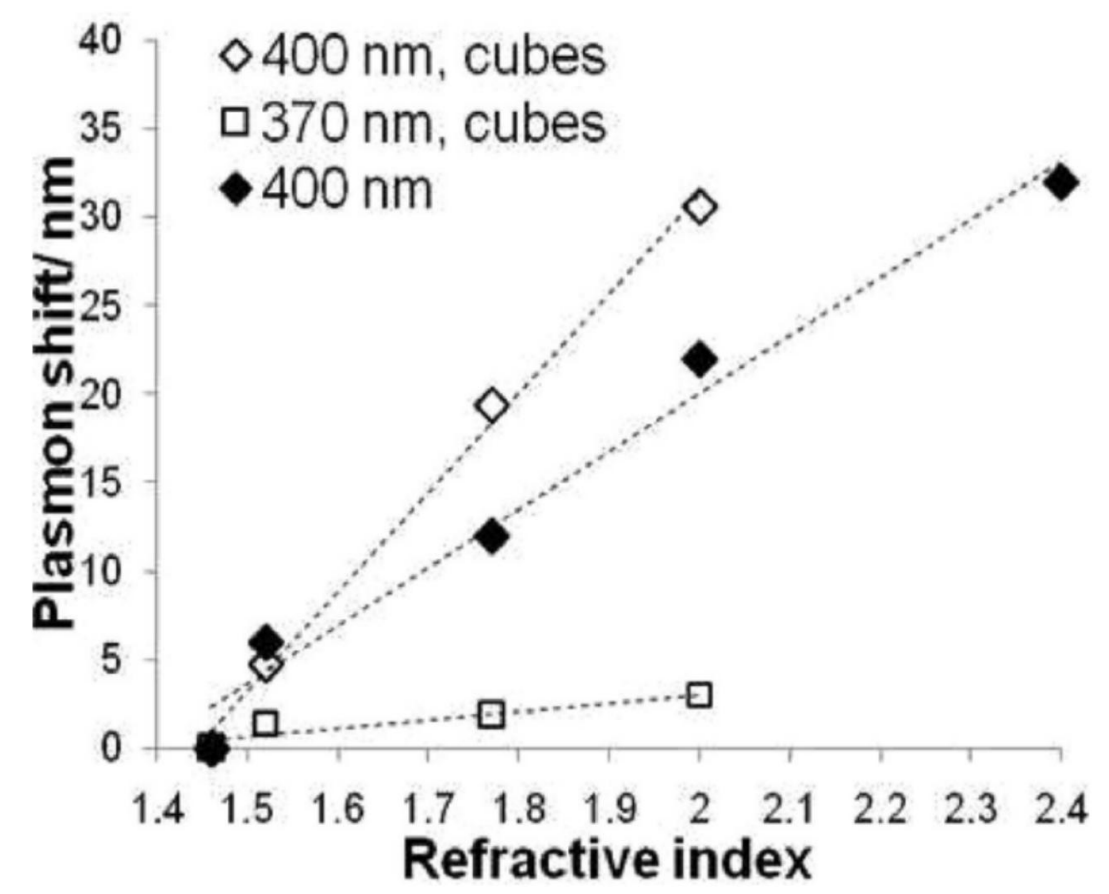

Figure 4.8. Plasmon shift of the dipolar (diamonds) and quadrupolar (squares, cubes only) bands in extinction spectra of supported 40nm Ag nanocubes (open symbols) and irregular Ag nanoparticles (filled symbols) as a function of support refractive index.

Since for a 40nm nanocube monolayer Q and D modes are well separated on a high refractive index substrate, such as $\mathrm{TiO}_{2}$ film, we tested the sensitivity of the spectra to the refractive index of the solution. It is expected that in contrast to the glass substrate the two plasmonic bands will remain separated on substrates with a higher refractive index even when immersed in liquid. This should allow capitalizing on the higher solution RI sensitivity of the Q mode. Results of the experiment are shown in Figure 4.5B. Indeed, the spectra of $\mathrm{TiO}_{2}$-supported nanocubes immersed in low refractive index solvent (water) show clearly a shoulder at $400 \mathrm{~nm}$ (fwhm $\approx 27 \mathrm{~nm}$ ) corresponding to the $\mathrm{Q}$ mode in addition to the main $\mathrm{D}$ mode at $432 \mathrm{~nm}$ (fwhm $\approx 54 \mathrm{~nm}$ ). As the refractive index increases, the shoulder gradually disappears. For pure ethylene glycol (refractive index 1.4318, 0.6 lower than the substrate) the shoulder is essentially absent. When the monolayer deposited on glass $(n=1.52)$ is immersed in water $(\mathrm{n}=1.33)$ the RI difference between the substrate and the solution is 
already less than 0.2 units. Therefore, we conclude that for $40 \mathrm{~nm}$ nanocubes it is necessary to have a refractive index difference of at least 0.7 between the substrate and the solvent if we are to observe and use the Q mode for RI sensing.

Despite the fact that the Q mode essentially disappears for high RI solutions, peak fitting of the $\mathrm{TiO}_{2}$-supported 40nm nanocube substrate spectra was attempted. Sensitivities of Q and D modes to the solution refractive index change were estimated to be $57 \mathrm{~nm} / \mathrm{RIU}$ for D, 90nm/RIU for Q mode, and 29nm/RIU for the band at 350nm (Figure 4.5B). The corresponding FOMs are 1.3, 3.3, and 2.9. The figure of merit obtained for the Q mode is greater than that calculated for the glass- supported substrate (Table 4.2) and is very close to that of the colloidal solution (Table 4.1). It is obvious that the inability to separate the two peaks in liquid prevented us from calculating the RIS and FOM for the Q mode more precisely. It is expected that substrates with even higher refractive indices would help in separating the two bands. In such a case an even higher FOM for the supported monodisperse nanocube monolayers can be achieved.

It is interesting to note, that the intensity of the Q band in UV-vis spectra increases with angle between the substrate normal and the direction of light (Figure 4.9). When the angle increases excitation of out-of-plane charge oscillations becomes possible. ${ }^{38,44}$ At the same time, it allows better interactions of light and dark modes, such as quadrupolar resonance. ${ }^{44}$ Therefore, it is possible that measurements performed at larger incident angles could provide another tool in optimizing RIS and FOM. 


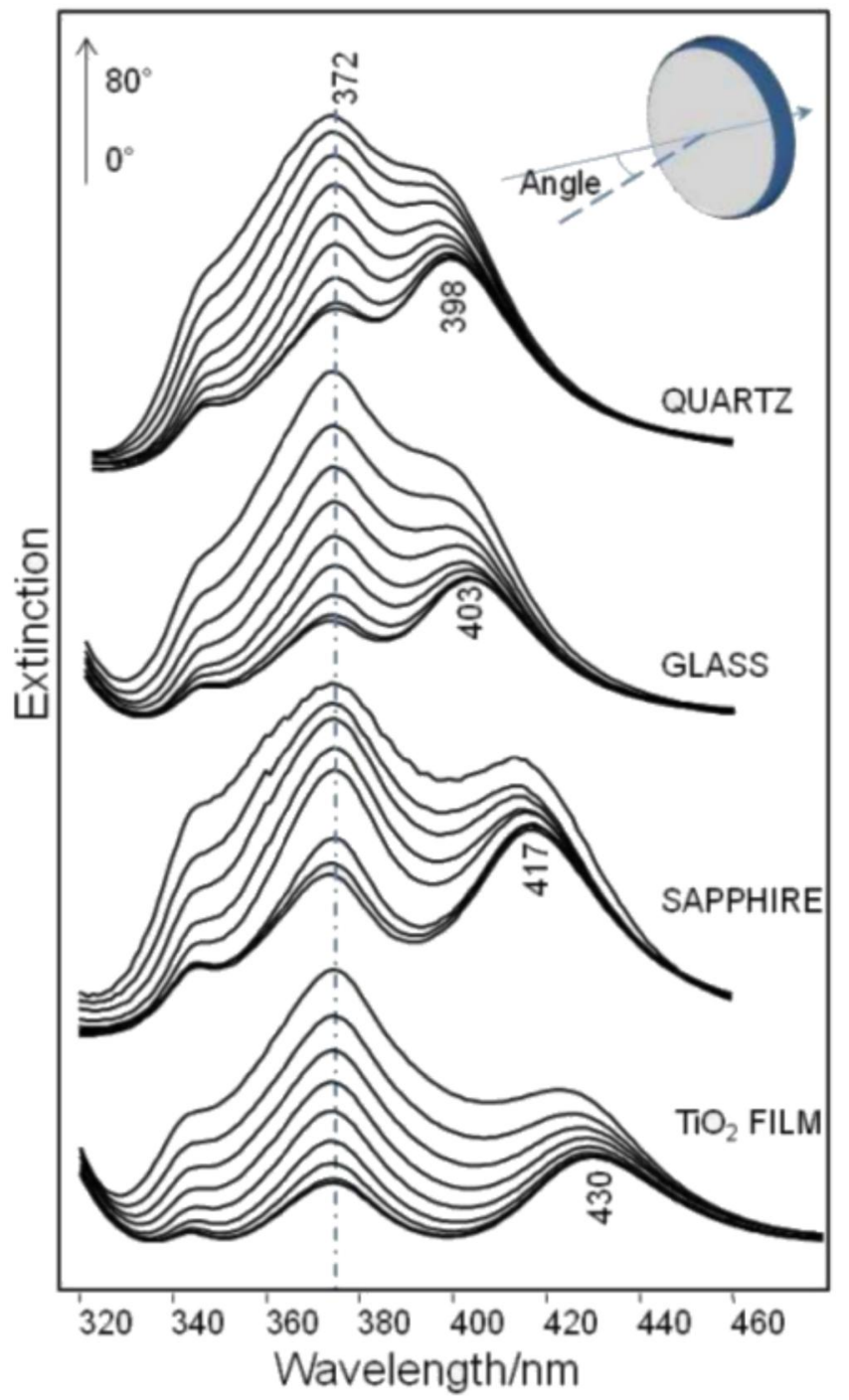

Figure 4.9. Angular dependence of UV-vis extinction spectra for 40nm nanocubes deposited on different substrates.

Finally, to determine the role of cubic geometry in the substrate-induced plasmon mode hybridization, appearance of new dipolar and quadrupolar modes, and consequent change in sensitivity to the refractive index, nanoparticles of the same size $(40-45 \mathrm{~nm}$, measured by AFM) but with a more spherical shape (truncated nanocubes based on the extinction spectra) were used to form monolayers on different substrates. UV-vis extinction spectra are presented in Figure 4.7B. The spectrum of a colloidal suspension of these nanoparticles contains a dipolar mode at $427 \mathrm{~nm}$, which is much broader than that of the 
nanocube suspension (Figure 4.7B and 4.7A top spectra, respectively). When deposited on a dielectric substrate two new bands appear. With increasing refractive index of the substrate the separation between the bands increases, which is similar to the nanocubes substrates. The degree of separation is, however, significantly smaller, resulting in a refractive index sensitivity of the lower energy band of $\sim 33 \mathrm{~nm} / \mathrm{RIU}$ (Figure 4.8 ). Such behaviour has previously been reported for spherical nanoparticles. ${ }^{28}$ The two bands were reported to be dipolar modes with orthogonal moments: the lower energy band corresponding to electron oscillations parallel to the surface and the higher energy band perpendicular to the surface. ${ }^{32}$ In contrast, the nanocube geometry allows different types of electron oscillations to be excited for the supported nanocube monolayers due to the larger contact area with the substrate, ${ }^{30,31}$ giving two bands with higher sensitivity to the refractive index changes of the substrate ( $\mathrm{D}$ band) or the solvent (Q band) than that for the spherical particle monolayers.

\subsection{Conclusions}

In this work we compared the refractive index sensing properties of silver nanocubes for three different sizes suspended in solution and supported in monolayers on dielectric substrates. We found that under certain conditions supported nanocube monolayers can have a refractive index sensitivity comparable to that of those in suspension. At the same time, the FOM can be even greater for substrate-supported cubes. This could be achieved if monodisperse nanocube samples and high refractive index substrates are used.

After submission of the manuscript we demonstrated that high refractive index substrates such as silicon can indeed lead to an improved figure of merit for a monolayer of silver nanocubes. ${ }^{45}$

\subsection{References}

1 Rycenga, M.; Cobley, C. M.; Zeng, J.; Li, W.; Moran, C. H.; Zhang, Q.; Qin, D.; Xia, 
Y. Chem. Rev. 2011, 111, 3669-3712.

2 Morton, S. M.; Silverstein, D. W.; Jensen, L. Chem. Rev. 2011, 111, 3962-3994.

3 Lee, P. C.; Meisel, D. J. Phys. Chem. 1982, 86, 3391-3395.

5 Ianoul, A.; Bergeron, A. Langmuir 2006, 22, 10217-10222.

6 Parfenov, A.; Gryczynski, I.; Malicka, J.; Geddes, C.; Lakowicz, J. R. J. Phys. Chem. B 2003, 107, 8829-8833.

7 Anger, P.; Bharadwaj, P.; Novotny, L. Phys. Rev. Lett. 2006, 96, 113002.

8 Rigneault, H.; Capoulade, J.; Dintinger, J.; Wenger, J.; Bonod, N.; Popov, E.; Ebbesen, T. W.; Lenne, P.-F. Phys. Rev. Lett. 2005, 95, 117401.

9 Hartstein, A.; Kirtley, J. R.; Tsang, J. C. Phys. Rev. Lett. 1980, 45, 201-204.

10 Chen, C. K.; de Castro, A. R. B.; Shen, Y. R. Phys. Rev. Lett. 1981, 46, 145-148.

11 Mayer, K. M.; Hafner, J. H. Chem. Rev. 2011, 111, 3828-3857.

12 Galush, W. J.; Shelby, S. A.; Mulvihill, M. J.; Tao, A.; Yang, P.; Groves, J. T. Nano Lett. 2009, 9, 2077-2082.

13 Anker, J. N.; Hall, W. P.; Lyanders, O.; Shah, N. C.; Zhao, J.; Van Duyne, R. Nat. Mater. 2008, 7, 442-453.

14 Zou, S.; Janel, N.; Schatz, G. C. J. Chem. Phys. 2004, 102, 10871-10875.

15 Auguie, B.; Barnes, W. L. Phys. Rev. Lett. 2008, 101, 143902.

16 Halas, N. J.; Lai, S.; Chang, W.-S.; Link, S.; Nordlander, P. Chem. Rev. 2011, 111, 3913-3961.

17 Giannini, V.; Fernandez-Dominguez, A. I.; Heck, S. C.; Maier, S. A. Chem. Rev. 2011, 111, 3888-3912.

18 Seok, T. J.; Jamshidi, A.; Kim, M.; Dhuey, S.; Lakhani, A.; Choo, H.; Schuck, P. J.; Cabrini, S.; Schwartzberg, A. M.; Bokor, J.; Yablonovitch, E.; Wu, M. C. Nano Lett. 2011, 11, 2606-2610.

19 Liu, N.; Hentschel, M.; Weiss, T.; Alivisatos, A. P.; Giessen, H. Science 2011, 332, $1407-1410$. 
20 Kelly, K. L.; Coronado, E.; Zhao, L. L.; Schatz, G. C. J. Phys. Chem. B 2003, 107, 668-677.

21 Barbosa, S.; Agrawal, A.; Rodriguez-Lorenzo, L.; Pastoriza-Santos, I.; Alvarez-Puebla, R. A.; Kornowski, A.; Weller, H.; Liz-Marzan, L. M. Langmuir 2010, 26, 1494314950.

22 Mulvaney, P. Langmuir 1996, 12, 788-800.

23 Burda, C.; Chen, X.; Narayanan, R.; El-Sayed, M. A. Chem. Rev. 2005, 105, 10251102.

24 Sosa, I. O.; Noguez, C.; Barrera, R. G. J. Phys. Chem. 2003, 107, 6269-6275.

25 Tao, A.; Sinsermsuksakul, P.; Yang, P. Angew. Chem., Int. Ed. 2006, 45, 4597-4601.

26 Kumbhar, A. S.; Kinnan, M. K.; Chumanov, G. J. Am. Chem. Soc. 2005, 127, 1244412445.

27 Zhou, F.; Li, Z.-Y.; Liu, Y.; Xia, Y. J. Phys. Chem. C 2008, 112, 20233-20240.

28 Knight, M. K.; Wu, Y.; Lassiter, J. B.; Nordlander, P.; Halas, N. J. Nano Lett. 2009, 9, 2188-2192.

29 McMahon, J. M.; Wang, Y.; Sherry, L. J.; Van Duyne, R. P.; Marks, L. D.; Gray, S. K.; Schatz, G. C. J. Phys. Chem. C 2009, 113, 2731-2735.

30 Sherry, L. J.; Chang, S.-H.; Schatz, G. C.; Van Duyne, R. P.; Wiley, B. J.; Xia, Y. Nano Lett. 2005, 5, 2034-2038.

31 Zhang, S.; Bao, K.; Halas, N. J.; Xu, H.; Nordlander, P. Nano Lett. 2011, 11, 16571663.

32 Ringe, E.; McMahon, J. M.; Sohn, K.; Cobley, C.; Xia, Y.; Huang, J.; Schatz, G. C.; Marks, L. D.; Van Duyne, R. P. J. Phys. Chem. C 2010, 114, 12511-12516.

33 Henry, A.-I.; Bingham, J. M.; Ringe, E.; Marks, L. D.; Schatz, G. C.; Van Duyne, R. P. J. Phys. Chem. C 2010, 115, 9291-9305.

34 Mahmoud, M. A.; El-Sayed, M. A. Nano Lett. 2011, 11, 946-953.

35 Skrabalak, S. E; Au, L.; Li, X.; Xia, Y. Nat. Protoc. 2007, 2, 2182-2190.

36 Ahamad, N.; Ianoul., A. J. Phys. Chem. C 2011, 115, 3587-3594.

37 Chem, H.; Kou, X.; Yang, Z.; Ni, W.; Wang, J. Langmuir 2008, 24, 5233-5237. 
38 Lee, Y. H.; Chen, H.; Xu, Q.-H.; Wang, J. J. Phys. Chem C 2011, 115, 7997-8004.

39 Siekkinen, A. R.; McLellan, J. M.; Chen, J.; Xia, Y. Chem. Phys. Lett. 2006, 432, 491496.

40 Lassiter, J. B.; Aizpurua, J.; Hernandez, L. I.; Brandl, D. W.; Romero, I.; Lal, S.; Hafner, J. H.; Nordlander, P; Halas, N. J. Nano Lett. 2008, 8, 1212-1218.

41 Slaughter, L. S.; Wu, Y.; Willingham, B. A.; Nordlander, P.; Link, S. ACS Nano 2010, 4, 4657-4666.

42 Zuloaga, J.; Prodan, E.; Nordlander, P. Nano Lett. 2009, 9, 887-891.

43 Goldys, E. M.; Calander, N.; Drozdowicz-Tomsia, K. J. Phys. Chem. C 2011, 115, 676-682.

44 Hao, F.; Sonnefraud, Y.; Van Dorpe, P.; Maier, S. A.; Halas, N. J.; Nordlander, P. Nano Lett. 2008, 8, 3983-3988.

45 Bottomley, A.; Ianoul, A. ChemPhysChem 2011, 12, 2919-2614. 


\section{Chapter 5}

Fine tuning of plasmonic properties of monolayers of weakly interacting silver nanocubes on thin silicon film

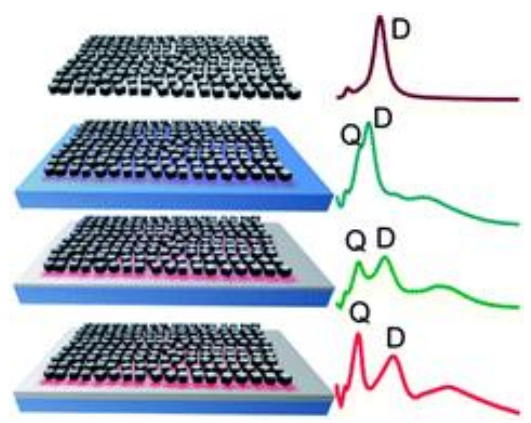


Modified from original manuscript published as:

Bottomley, A., Prezgot, D., Staff, A. and Ianoul, A., Fine tuning of plasmonic properties of monolayers of weakly interacting silver nanocubes on thin silicon films. Nanoscale 2012, 4, 6374-6382.

doi:10.1039/c2nr31885g

Department of Chemistry, Carleton University, 1125 Colonel By Drive, Ottawa, Ontario, Canada, K1S 5B6

\subsection{Abstract}

Plasmonic properties, such as refractive index sensitivity (RIS), surface enhancement of the Raman signal (SERS), fluorescence quenching, and photocatalytic activity, of monolayers of weakly interacting monodisperse silver nanocubes were qualitatively modified in a very well controlled manner by supporting them on thin silicon films with varying thickness. Such fine tunability is made possible by the strong dependence of the nanocube dipolar (D) and quadrupolar (Q) plasmon mode hybridization on the refractive index of the supporting substrate. By increasing the Si film thickness from zero to $\sim 25 \mathrm{~nm}$ we were able to "shift" the D resonance mode by up to $200 \mathrm{~nm}$ for $\sim 80 \mathrm{~nm}$ cubes without significantly affecting the Q mode. The silicon supported nanocubes showed a significant improvement in RIS via the Q mode with a figure of merit greater than 6.5 and about an order of magnitude enhancement of the SERS signal due to the stronger electric field created by the D mode. Such substrates also showed a $\sim 10$ times decrease in rhodamine $6 \mathrm{G}$ fluorescence as well as the rates of amorphous carbon formation. The study proposes a new way to design and engineer plasmonic nanostructures.

\subsection{Introduction}

Since the development of methods for efficient, facile, reproducible, and inexpensive synthesis of large quantities of monocrystalline and highly monodisperse silver nanocubes with sharp corners, ${ }^{1}$ numerous theoretical and experimental studies on a single nanocube ${ }^{2-4}$ 
or an ensemble of nanocubes ${ }^{5-9}$ have revealed their unique optical properties. Superior refractive index sensitivity (RIS) ${ }^{2,5}$ and Raman signal enhancement ${ }^{10,11}$ have been demonstrated and the strong dependence on the refractive index of local environment has been utilized to monitor a molecular binding event on membrane surfaces. ${ }^{12}$ In this application ensembles of cubes were supported for convenience. This is likely to be the case for most applications and as such the effect of a substrate on plasmonic properties becomes of crucial importance.

Effects of a substrate on plasmonic properties of metal nanoparticles have been investigated extensively. ${ }^{13-18}$ Recently it has been shown that for a single spherical metal particle on a dielectric support the degeneracy of the dipolar (D) localized surface plasmon resonance (LSPR) mode is lifted and two bands emerge corresponding to two orthogonal electron oscillations (parallel and perpendicular to the substrate plane) instead of a single band for a nanoparticle in a solution. ${ }^{13}$

When a single gold nanorod was supported on silicon, interference between the octupolar and quadrupolar plasmon resonances was observed leading to the appearance of Fano resonance with a very strong dependence on the nanorod-substrate spacing. ${ }^{19}$ For disk shaped silver nanoparticles on silicon, excitation of two different types of resonant modes was reported: the shorter wavelength resonance excited at the nanoparticle top, while the longer wavelength was excited at the silver-substrate interface. ${ }^{20}$ Finally, for a single silver nanocube supported on different dielectric surfaces a new band was observed at a shorter wavelength than dipolar LSPR. ${ }^{2}$ The band was identified as corresponding to oscillations of electrons at the opposite side of the nanocube from the substrate and therefore insensitive to the substrate, while the dipole was found to correspond to electron oscillations at the nanocube-substrate interface and sensitive to the substrate refractive index. ${ }^{2}$ Later, the effect 
was theoretically explained as a result of plasmon mode hybridization between a bright dipolar and a dark quadrupolar (Q) mode. ${ }^{21}$ Interference between the two modes was also predicted to lead to Fano resonances. More recently we have shown that not only single nanocubes, but also ensembles of nanocubes in monolayers demonstrate a similar plasmonic behaviour if the cubes are well separated by an inert spacer and a highly monodisperse sample is used. ${ }^{22}$ We also capitalized on the fact that an ensemble of nanocubes can be considered a good model of a single nanocube due to the relatively weak dependence of the D mode wavelength on the nanocube size. ${ }^{4}$ The degree of plasmon mode hybridization was found to be dependent, among other factors, on the refractive index of the substrate. ${ }^{21}$ Incremental increase in the substrate refractive index was shown experimentally as a way to control the plasmon mode hybridization. ${ }^{22}$ Substrates such as quartz, glass, or sapphire have monotonically changed the degree of hybridization. ${ }^{22}$ Yet, multiple studies on high refractive index substrates - silicon supported nanoparticles - have been conducted ${ }^{19,20,23-25}$ aiming at the development of plasmon-based optical and optoelectronic devices ${ }^{19,23}$ or increasing the efficiency of silicon solar cells..$^{20,24,25}$

Very recently we hypothesized that a thin nanometer scale silicon film deposited on a glass slide can be used to effectively control the degree of plasmon mode hybridization. ${ }^{26}$ In the present work we have systematically investigated the effect of such a thin silicon film on plasmonic properties of supported silver nanocube monolayers. We demonstrate that by varying the Si film thickness it becomes possible to fine-tune the plasmonic signature of the nanocube monolayers leading to some novel observations.

\subsection{Experimental}

\section{Nanocube synthesis and purification:}

The nanocube sample was synthesized and purified as described previously.,22 


\section{Monolayer formation:}

Nanocube monolayer substrates were prepared using the Langmuir technique as reported previously. ${ }^{6}$ Nanocube samples were mixed with 1,2-dioleoyl-sn-glycero-3phosphocholine (DOPC) prior to the monolayer formation to prevent nanoparticle aggregation resulting in strong interparticle dipole-dipole coupling ${ }^{27}$ and complicating spectral analysis. UV-vis spectroscopy and AFM imaging were used to monitor the degree of nanocube aggregation.

After several compression/expansion cycles the nanocube-DOPC monolayers were transferred onto substrates cleaned by sonication in methanol, at surface pressures corresponding to the gas phase of the monolayer by vertical upward dipping at $2 \mathrm{~mm} / \mathrm{min}$ at room temperature.

The substrates were typically $1 \times 2$ inch $^{2}$ microscopic glass slides, half-bare and halfcoated with a thin amorphous silicon film by the physical vapour deposition at the Carleton University Microfabrication Facility. Silicon film thickness was confirmed by AFM measurements of the silicon film edge.

\section{UV-vis measurement:}

UV-vis and refractive index sensitivity (RIS) measurements of the monolayers deposited on various substrates were performed as previously described. ${ }^{22}$ Silicon background subtraction and spectral deconvolution were performed with GRAMS/AI spectral data processing software using the appropriate number of mixed GaussianLorentzian functions and a linear baseline to achieve the best fit to the original trace while minimizing $\chi^{2}$ values. Iregularities in particle distribution and/or the substrate can influence the peak widths and positions observed in the extinction spectrum, so a position corresponding the most occurring spectrum or statistical mode was chosen as the initial 
spectra and kept in place for a whole series.

\section{Topography measurement}

The topography of the NC monolayer was obtained using an Ntegra (NT-MDT, Russia) atomic force microscope in semi-contact mode in air at $23^{\circ} \mathrm{C}$ with $1024 \times 1024$ points per image. A $100 \times 100 \mu \mathrm{m}^{2}$ scanner (Ntegra) and cantilevers with single crystal silicon tips (135 mm long, $1.7 \mathrm{~N} \mathrm{~m}^{-1}$ spring constant, resonance frequency $90 \mathrm{kHz}$, NT-MDT) were used for all topographic measurements. The typical scan rate was $0.5 \mathrm{~Hz}$. AFM images were further processed by Nova image processing software.

\section{SERS measurement:}

For SERS measurements the substrates (half-bare glass, half- silicon film on glass) were immersed in $1 \mathrm{mM}$ aqueous solution of rhodamine $6 \mathrm{G}$ for 30 minutes followed by rinsing in water and drying in air. Measurements of SERS spectra were performed in a $135^{\circ}$ geometry using a single grating monochromator (Jobin Yvon, focal length 640mm) equipped with a liquid nitrogen cooled CCD camera (Princeton Instruments) and a notch filter to remove the excitation wavelength. An argon-krypton ion laser system (Coherent), operating at $458,488,514,568$ or $647 \mathrm{~nm}$ excitation wavelengths was used for excitation. The spectral resolution of the instrument was $\sim 4 \mathrm{~cm}^{-1}$. The SERS spectra were collected by WinSpec/32 software and further processed by GRAMS/AI spectral data processing software. Cyclohexane was used for Raman shift calibration. Steady state SERS spectra were obtained on glass and silicon sides of the substrate in a sequence by averaging nine spectra collected on different areas of the sample. The accumulation time was 60s and the laser power was maintained below $1 \mathrm{~mW}$. Three spectra were measured in each spot to determine the degree of photodecomposition. For SERS kinetic measurements a sequence of 60 separate spectra with 1s accumulation time each were collected and averaged over three areas of the sample. 


\subsection{Results and Discussion}

\section{Monolayers of weakly interacting nanocubes as a good model to probe the} substrate effect:

The Langmuir-Blodgett technique is a convenient way to assemble nanoparticles, including metal nanorods and silver nanocrystals in monolayers. ${ }^{9}$ We can use the surface pressure in such water supported nanocube monolayers to adjust the separation between the cubes and observe some new optical properties. ${ }^{6}$ However reproducibility of such monolayers is known to be rather poor due to the tendency of nanocubes to aggregate. To address this issue we have recently developed a methodology utilizing surface active molecular spacers, such as phospholipids existing in liquid expanded phase in Langmuir monolayers, for example DOPC. ${ }^{27}$ The lipid molecules serve to separate the cubes and to prevent the aggregation even at fairly high surface pressures, therefore enabling reproducible experimental conditions. As a result, composite nanocube-lipid monolayers can be prepared and compared experimentally on various substrates. In this work such monolayers were transferred by the Langmuir technique onto slides with half of the slide bare and another half being coated with a thin film of amorphous silicon. Such an approach allows us to: (i) compare the properties of the exact same monolayer on two different substrates - glass and thin silicon film, and (ii) use the glass slide deposited monolayers as a reference for quantitative comparison of monolayers on various silicon films.

As an example, an AFM topographical image of a monolayer consisting of relatively small (35-40nm) nanocubes mixed with DOPC, transferred at a low surface pressure of $1.5 \mathrm{mN} / \mathrm{m}$, corresponding to the gas phase of the monolayer, shows well separated, monodisperse nanoparticles (Fig. 5.1A) 

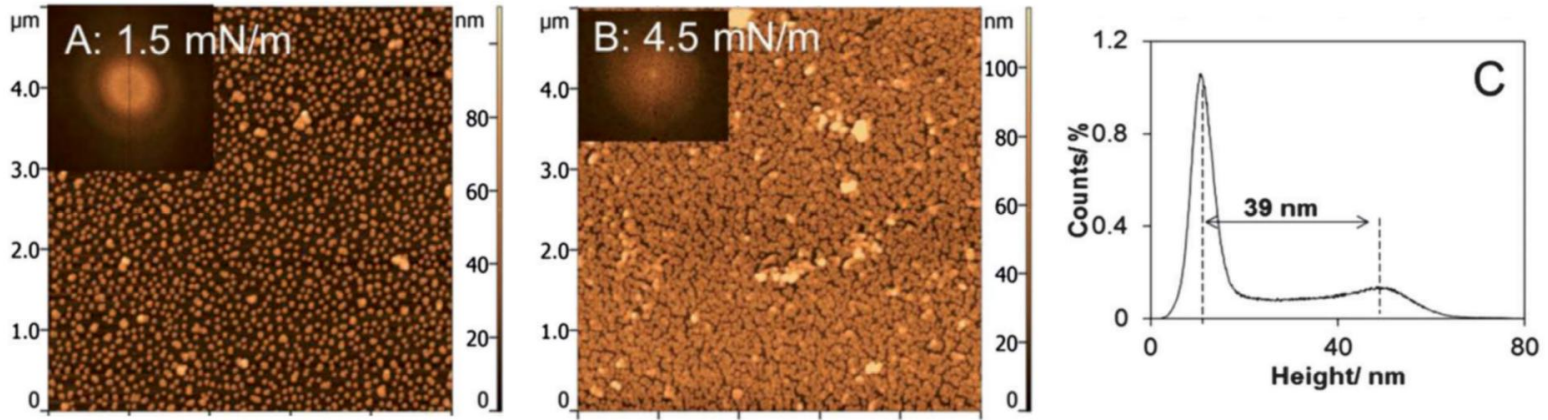

Figure 5.1. AFM topography images of a monolayer of 35-40nm silver nanocubes-DOPC deposited by the Langmuir technique on a $15 \mathrm{~nm}$ thick silicon film at (A) $1.5 \mathrm{mN} / \mathrm{m}$ and (B) $4.5 \mathrm{mN} / \mathrm{m}$. Insets show the corresponding fast Fourier transformation (FFT) images. Two concentric rings in FFT of the $1.5 \mathrm{mN} / \mathrm{m}$ sample indicate the short range order with a nearest neighbour distance (center-to-center) of $\sim 120 \mathrm{~nm}$ and nanocube size of $\sim 40 \mathrm{~nm}$. The short range order disappears at $4.5 \mathrm{mN} / \mathrm{m}$ according to the FFT image. (C) Topographical analysis of the monolayer deposited at $1.5 \mathrm{mN} / \mathrm{m}$ indicating the average height of the nanocubes.
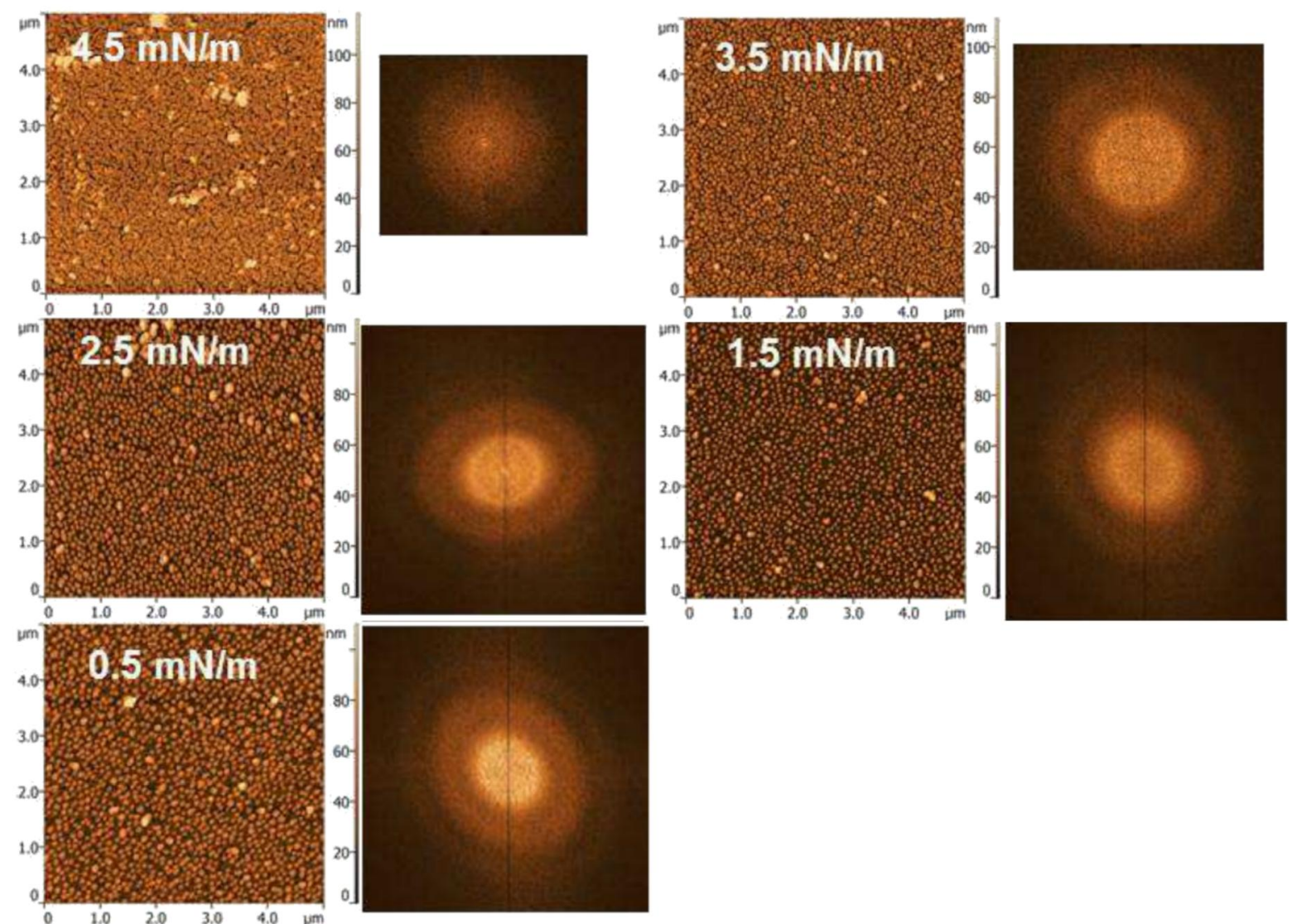

Figure 5.2. Left column: AFM topography images of monolayers of 35-40nm silver nanocubes/DOPC deposited on a 15nm thick silicon film at various surface pressures, as indicated. Right column: corresponding Fast Fourier transformation images corresponding to the images on the left side. Concentric circles indicate the presence of short range order (middle to middle, the smallest circle), and the particle size (second circle) respectively. The third concentric circle indicates the separation between the particles (side to side). Short range order disappears at high surface pressure of $4.5 \mathrm{mN} / \mathrm{m}$. 
The separation between the cubes is well controlled, as confirmed by the fast Fourier transform (FFT) image shown as an inset in Fig. 5.1A. The two periods in FFT correspond to the particle size (outer circle) and a center-to-center inter-particle separation (inner circle). Occasionally a third period corresponding to the inter-particle side-to-side separation can be observed (Fig. 5.2). A better indication of the nanocube size and size homogeneity is obtained from the height measurements as indicated in Fig. 5.1C due to the higher axial resolution of the instrument.

With increasing surface pressure the interparticle separation decreases (Fig. 5.2) and at a certain pressure threshold (dependent on the nanocube properties, nature and amount of lipid added) aggregation occurs. In the case of the monolayer shown in Fig. 5.1 this occurs at a pressure around $4.5 \mathrm{mN} / \mathrm{m}$, when according to FFT image, the only period observed is the nanocube size (Fig. 5.1B, inset). The corresponding extinction spectra of the monolayers clearly show the transition (Fig. 5.3). A long wavelength band, corresponding to interparticle dipole-dipole coupling, appears at 600-700nm and becomes very strong at $4.5 \mathrm{mN} / \mathrm{m}$. The band is observed on both glass and silicon parts of the substrates (Fig. 5.3 left and right panels respectively). At low surface pressure, when according to FFT images the interparticle separation is maintained above the cube size the monolayer can be considered as that of nanocubes very weakly interacting with each other and therefore represents a very good model to study the effect of substrate only on their individual plasmonic properties.

\section{Extinction spectra of silver nanocube monolayers on silicon films:}

It was predicted theoretically ${ }^{21}$ and we have shown this recently experimentally ${ }^{22,26}$ that for a silver nanocube on a dielectric substrate plasmon mode hybridization occurs with the appearance of two hybrid modes: dipolar (D) and quadrupolar (Q). The degree of mode hybridization was shown to depend on the substrate refractive index. ${ }^{21}$ One way to vary the 
refractive index of a substrate is to use thin nanometer scale silicon films deposited on a glass slide with the film thickness proportional to its effective refractive index. ${ }^{26} \mathrm{We}$ performed a comprehensive investigation of plasmon mode hybridization in monolayers of non-interacting silver nanocubes on such thin silicon films.

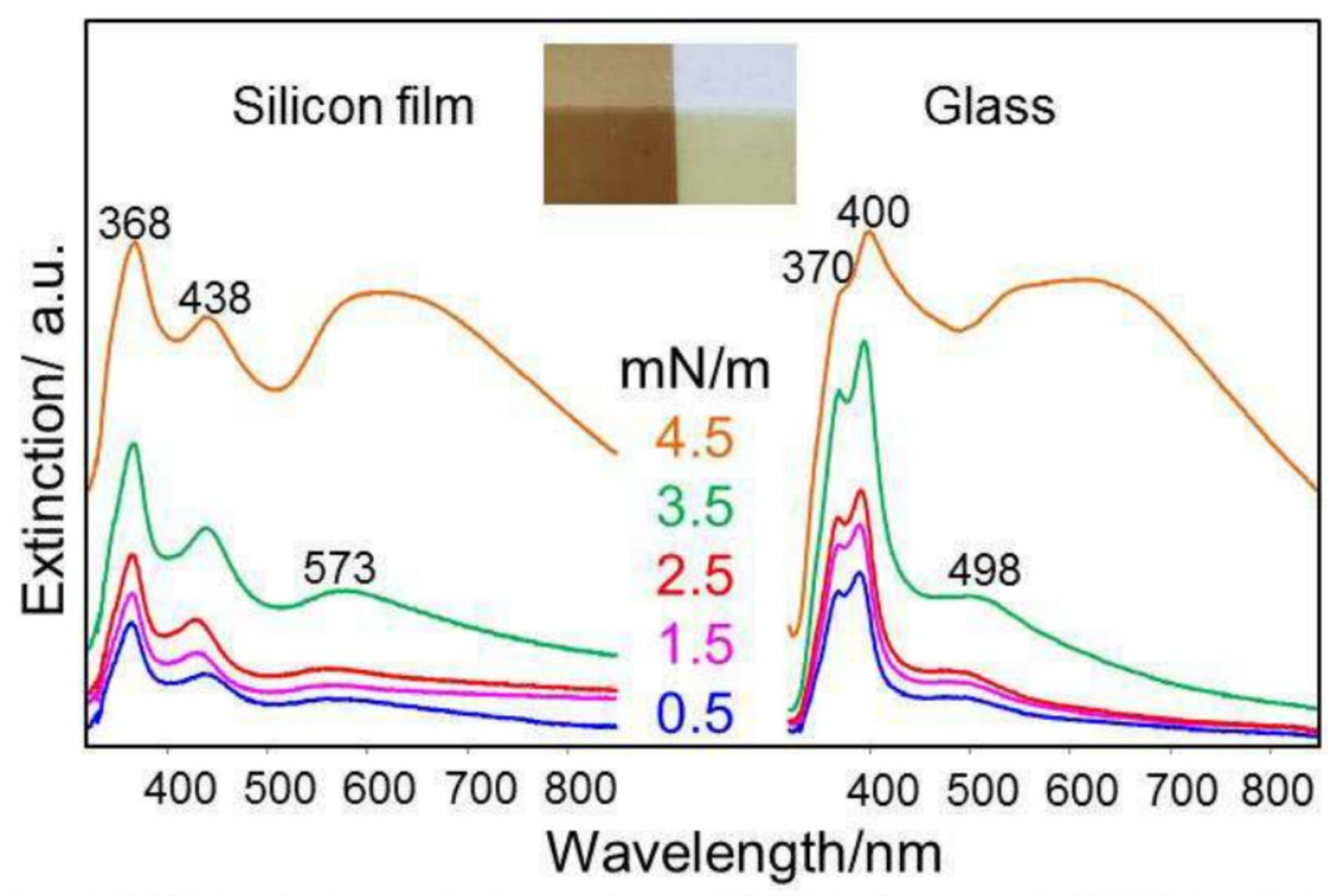

Figure 5.3. UV-vis extinction spectra for monolayers of 35-40nm silver nanocube/ DOPC deposited at various surface pressures on a $15 \mathrm{~nm}$ silicon film (left) or glass slide (corresponding to figure 5.2 images). Deposition was performed simultaneously onto glass and silicon sides of the substrate. The strong dipole-dipole coupling peak becomes dominant at high surface pressure of $4.5 \mathrm{mN} / \mathrm{m}$. The photo shows s slide with silicon film on the left hand side. The bottom part of the slide is coated with the nanocube/DOPC monolayer.

Fig. 5.4 shows UV-vis extinction spectra of four different nanocube samples. The sizes of the nanocubes were determined from their extinction spectra in ethanol (Fig. 5.4, black lines) and AFM images to be around 35-40nm (A), 40-45nm (B), 75- 80nm (C) and 80-85nm (D). A slight variation in sample morphology between the samples C and D is expected, with the sample D containing a fraction of truncated cubes, indicated by the strong band at 380nm. ${ }^{28,29}$ Extinction spectra of nanocube monolayers on thin silicon films are 
shown as blue, green, orange, and red curves for $0,10,15$, and $25 \mathrm{~nm}$ respectively.

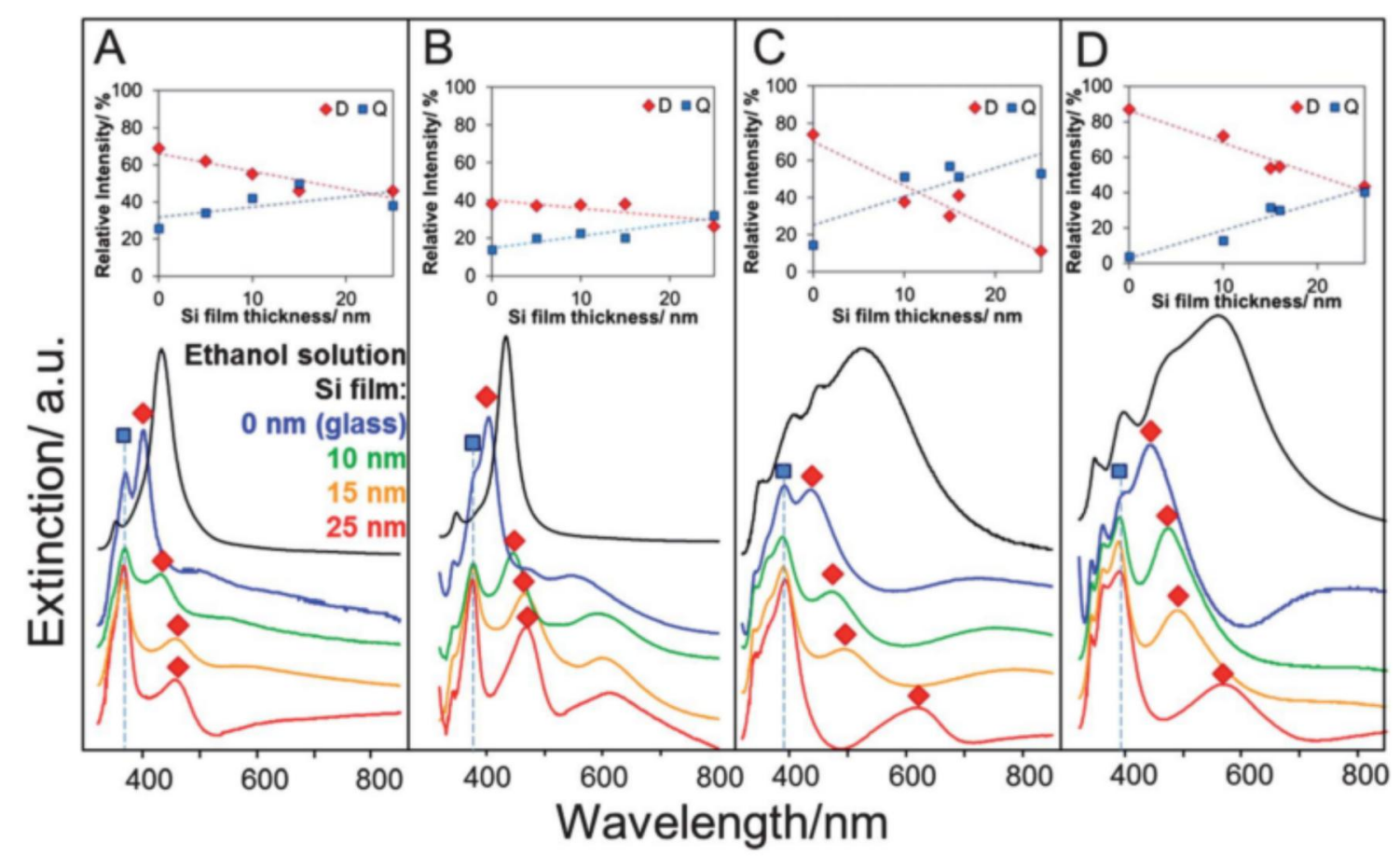

Figure 5.4. Extinction spectra of silver nanocubes in ethanol (black lines) and silver nanocubeDOPC monolayers deposited on silicon films of 0 (glass, blue lines), 10 (green), 15 (orange) and 25nm (red) thickness for (A) 35-40nm cubes; (B) 40-45nm cubes; (C) 75-80nm cubes, and (D) 80$85 \mathrm{~nm}$ cubes. Blue squares indicate the positions of the quadrupolar peaks and red rhombs - dipolar peaks. Graphs at the top of each panel indicate the relative quadrupolar (Q) or dipolar (D) integral extinction intensities as a function of Si film thickness. Results of spectral fitting are presented in Fig. 5.5 .

The first sample of the smallest nanocubes (Fig. 5.4A) has a dipolar LSPR with a maximum peak at $431 \mathrm{~nm}$ in solution and a weak but noticeable peak at $350 \mathrm{~nm}$. When deposited on a substrate, as expected two hybrid bands appear in place of the single dipolar band: a quadrupolar at 389nm indicated as blue squares and a dipolar at $400 \mathrm{~nm}$ marked with red rhombs. The band at 350nm is still present, but only as a shoulder to the Q band. Weak dipole-dipole coupling can be also seen at longer wavelengths. With increasing silicon film thickness the D band red-shifts until the silicon film thickness reaches 15nm (Fig. 5.6), whereas the spectral position of the Q band does not change. 


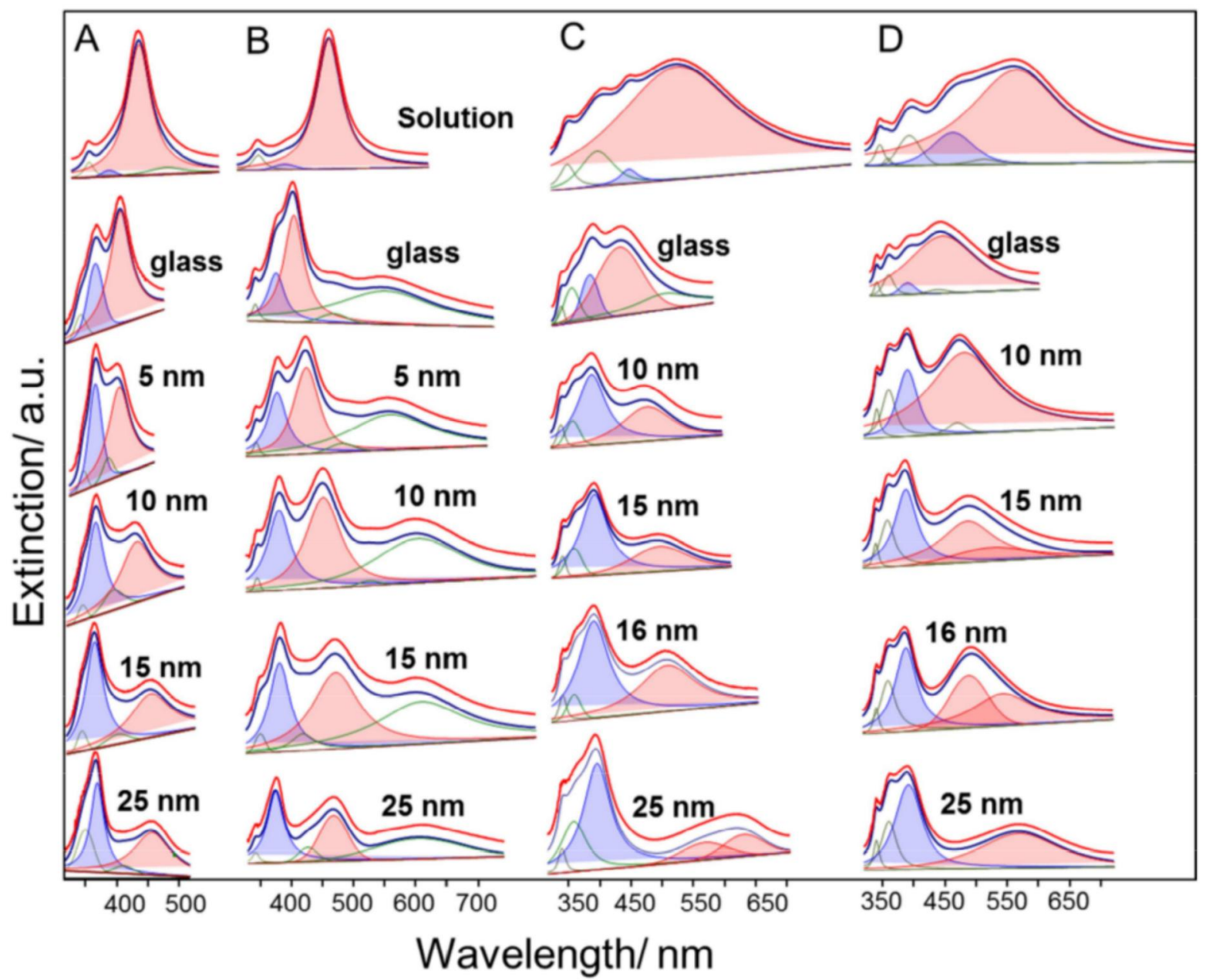

Figure 5.5. Results of extinction spectra deconvolution for silver nanocubes in ethanol solution (the top most spectra) and for nanocube/ DOPC monolayers deposited on different substrates in air. Series in columns correspond to samples A, B, C, and D (from left to right) in Figure 5.4. Red thick curves represent experimental data. Dark blue thick curves represent results of spectral fitting. Blue and red peaks correspond to quadrupolar and dipolar modes respectively. Silicon film thickness is indicated.

A similar trend is observed for the sample B. However, the D and Q bands are slightly red shifted compared to the sample A, and the band at 350nm can now be clearly resolved. This second sample shows a bit stronger interparticle interaction as indicated by a stronger dipole-dipole coupling peak at 550-650nm. As such, the D and Q bands are not well resolved on glass (Fig. 5.4B, blue spectrum). The hybrid dipolar band shift for this sample also saturates for $\sim 15 \mathrm{~nm}$ silicon films, and does not change for thicker films (Fig. 5.6). For bigger cubes, sample $C$, in addition to the $\mathrm{D}, \mathrm{Q}$, and a band at $350 \mathrm{~nm}$ a new band 
appears in solution at $\sim 370 \mathrm{~nm}$, corresponding to higher order resonance. 5,28,29 $^{2}$

For this sample of bigger cubes the same trend of the dipolar hybrid mode shifting with increasing silicon film thickness is observed as for smaller cubes, as well as invariance of the quadrupolar band position. Yet, the dipolar band shift does not saturate even for 25nm films (Fig. 5.6).

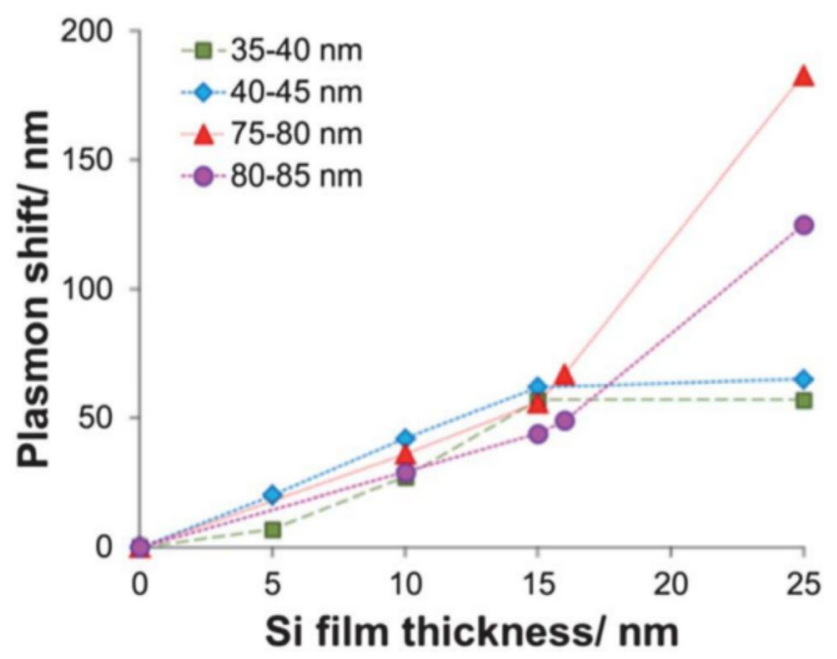

Figure 5.6. Position of the dipolar plasmon mode as a function of silicon film thickness for four different monolayers.

Finally, for even bigger cubes, sample D, the same trend of hybrid D band red shifting is observed. The only exception is the stronger intensity of the band at 370nm which is likely due to the presence of various crystalline morphologies.

The spectra obtained were then subjected to spectral fitting, with the results presented in Fig. 5.5 Positions of the dipolar bands extracted from such deconvolution for different Si film thicknesses are shown in Fig. 5.6, and the relative integral intensities for the dipolar and quadrupolar bands are shown as insets in Fig. 5.4. Several important observations can be made from the results of such fitting:

1. Above certain Si film thickness even the D hybridized mode becomes insensitive to the substrate change. Such film thickness is related to the nanocube size, and is equal 
approximately to the cube side half-length. The saturation of the D mode shift is a result of the distance dependence of the local electric field associated with this mode. ${ }^{30,31}$ The observations here are consistent with previous theoretical predictions of the mode electric field distribution, ${ }^{21}$ however, they represent first experimental demonstration of the extent of electric field associated with the nanocube dipolar mode.

2. The degree of $\mathrm{D}$ band shift depends on the quality of the sample, and is the greatest for pure cubic morphology, such as for $40-45 \mathrm{~nm}$ cubes. This is likely because the contact area between the particle and the silicon film is the greatest for cubes. The conclusion follows from comparing samples C and D. The first one has slightly smaller cubes, however, consistently shows greater plasmonic shifts for the dipolar mode (Fig. 5.6) since the second sample has a fraction of truncated geometries leading to smaller contact area even for bigger crystals.

3. The relative integral intensity of the hybrid $\mathrm{D}$ band decreases and that of the hybrid Q band increases with film thickness. This is likely due to the fact that as the thickness of the Si film increases, the substrate mediates a stronger interaction of the D mode and the Q mode such that the dipolar moment of the $\mathrm{D}$ mode becomes smaller and smaller and the opposite is true for the Q mode. The larger the dipolar moment, the stronger the mode can interact with incident light, i.e., a stronger extinction peak. This is a nice experimental observation of the substrate mediated plasmon hybridization.

4. Finally, the full width at half-maximum does not change significantly with the film thickness for all samples. At the same time, at certain film thicknesses it becomes impossible to fit the spectra to the simple set of peaks, as in solution (for example, Fig. 5.5 samples C 25nm, D 15, 16nm). We consider that to be mainly due to artifacts associated with spectra processing (baseline subtraction for thicker films) and stronger light scattering for bigger 
cubes. ${ }^{18}$ It is possible, however, that at these thicknesses interference between the hybrid D and Q modes takes place, ${ }^{21}$ potentially leading to Fano resonances. A more detailed investigation is required to observe the effect at a monolayer level.
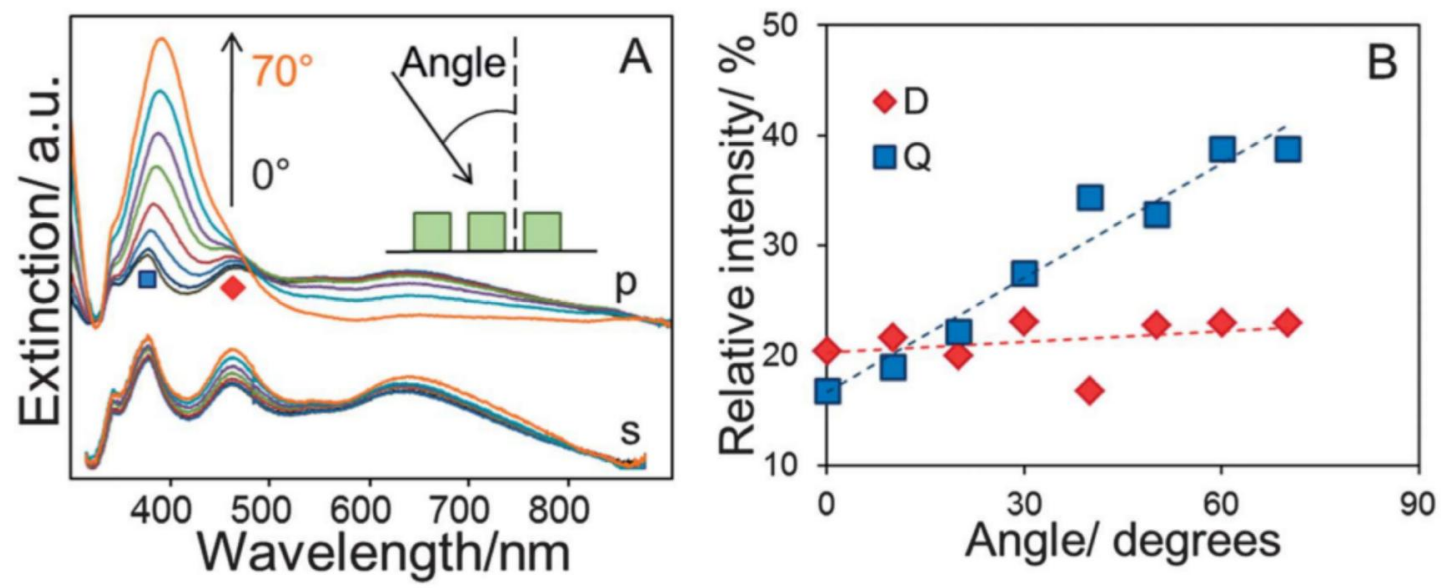

Figure 5.7. (A) Angle dependence of extinction spectra for the 40-45nm nanocube monolayer deposited on a $\sim 15 \mathrm{~nm}$ Si film for s- and p- polarized light. (B) Relative quadrupolar (Q) or dipolar (D) integral extinction intensities as a function of angle for $p$-polarized light.

Extinction of the quadrupolar hybridized mode shows also a very strong angle dependence for the p-polarized light (Fig. 5.7) with its intensity more than doubled when the angle is increased from 0 to 70 degrees. No such dependence for the dipolar mode for the spolarized light was detected and it could be explained by better interactions of light and dark modes, such as quadrupolar resonance, at large angles. ${ }^{32}$ Similar polarization/angle dependencies were previously observed for small silver nanoparticle on various substrates, ${ }^{17}$ such as annealed silver island films on glass, ${ }^{33}$ and more recently for gallium nanoparticles on sapphire. ${ }^{14}$

The strong dependence of the nanocube plasmonic properties on the silicon film thickness enables the fabrication of substrates with desired properties. In the following sections two applications are presented that capitalize on such fine tunability. 


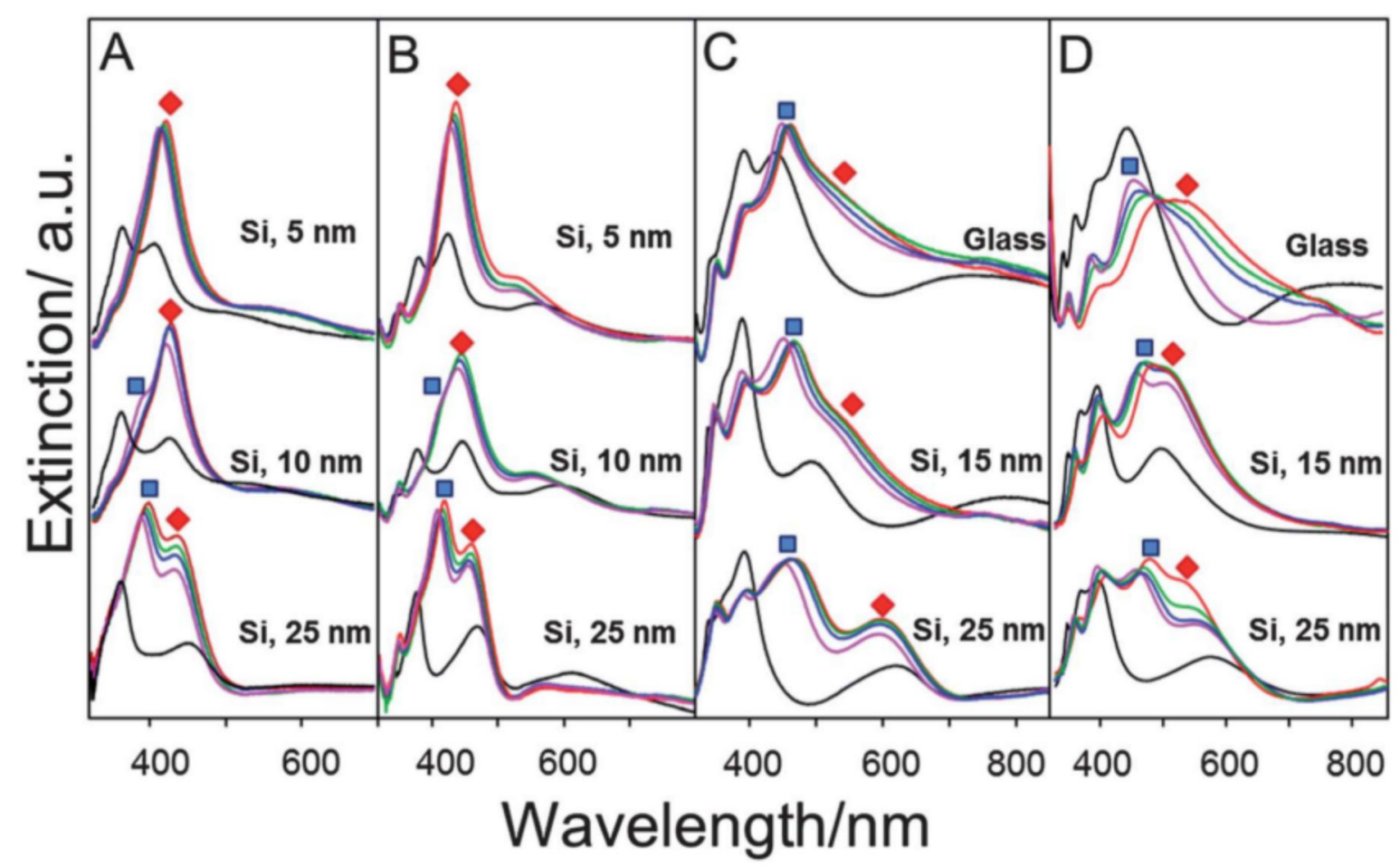

Figure 5.8. Refractive index sensitivities of supported silver nanocube monolayers on Si films. (A) 35-40nm cubes, (B) 40-45nm cubes, (C) 75-80nm cubes, and (D) 80-85nm cubes. Black lines represent extinction spectra in air; magenta, blue, green, and red in $\mathrm{H} 2 \mathrm{O}$-ethylene glycol 1/0, 2/1, $1 / 2$, and 0/1 mixtures respectively. Blue squares indicate the positions of the quadrupolar peaks and red rhombs - dipolar peaks for monolayers immersed in liquids.

\section{Silicon supported monolayers of non-interacting nanocubes can be optimized}

\section{for refractive index sensing:}

The strong dependence of the $\mathrm{D}$ and $\mathrm{Q}$ mode intensities and the $\mathrm{D}$ mode spectral position on the supporting substrate refractive index observed for silver nanocube monolayers enables the engineering of plasmonic nanostructures to probe refractive index changes in their environment. It has been shown that the hybrid Q mode is more sensitive to the refractive index of the surroundings than the $\mathrm{D}$ mode, mainly due to the location of associated electron oscillations at the side of the cubes away from the substrate. ${ }^{21,22}$ Yet, in solution the mode can be only observed when sufficient contrast (refractive index difference) between the substrate and the environment exists, greater than 0.7 units, ${ }^{22}$ thus setting the requirements for the minimum silicon film thickness. In addition, the Fano like interference 
between the two modes that occurs for a particular substrate refractive index value ${ }^{21}$ can bring about additional sensitivity to the quadrupolar mode.

Fig. 5.8 shows extinction spectra for four nanocube monolayer samples on thin silicon films in air (black lines) and in water-ethylene glycol mixtures by volume 1/0 (magenta), 2/1 (blue), 1/2 (green), and 0/1 (red). Blue squares indicate the positions of hybrid Q mode, whereas red rhombs indicate the D mode. Detailed deconvolution of the spectra (examples are shown in Fig. 5.9-5.11) was used to identify the following important general trends:

1. All bands redshift with increasing solution refractive index. By plotting the plasmon shifts as a function of solution refractive index (Fig. 5.12A and B) RIS values of various spectral bands could be calculated (Tables 5.1 and 5.2). These values depend not only on the cube size but also on the supporting silicon film thickness.

2. The quadrupolar/dipolar band splitting increases with nanocube size and the refractive index difference between the substrate and the surroundings.

3. For certain conditions only the dipolar band can be clearly identified, whereas the quadrupolar is very weak (Fig. 5.8A and B for silicon 5nm). In such a case the dipolar mode shows the greatest RIS. The figures of merit (FOM) calculated as a ratio between the RIS and the full width at half-maximum (FWHM) of the bands are found to be in the 0.1-4.0 range (Tables 5.1-5.4). In almost all cases the RIS and FOM values for the quadrupolar modes are the greatest. 

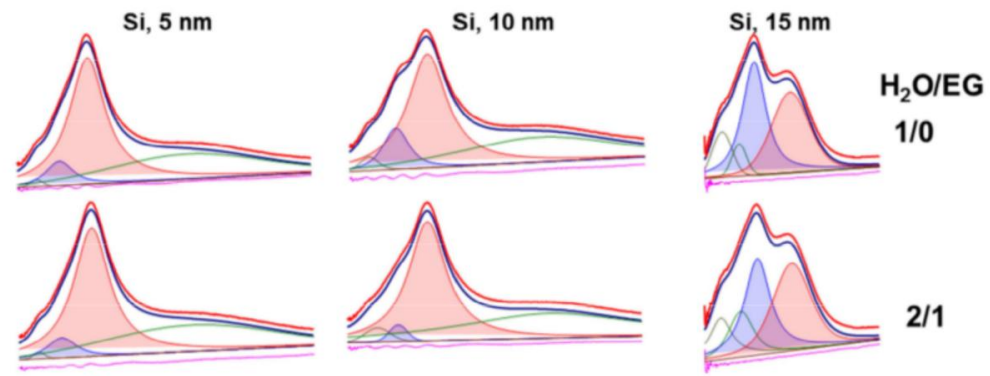

$2 / 1$
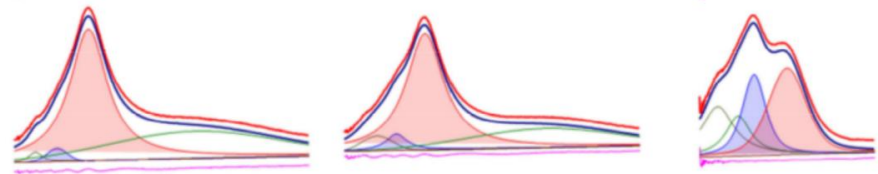

$1 / 2$
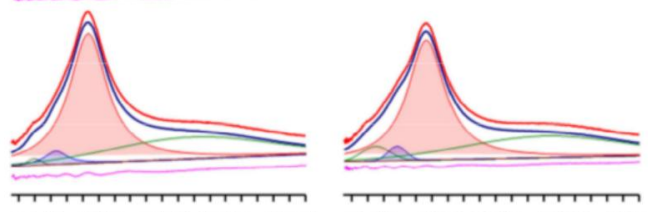

340420500580660 Wavelength/nm

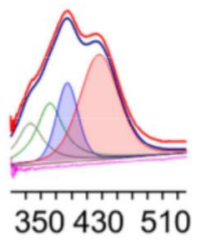

$0 / 1$

Figure 5.9. Results of extinction spectra deconvolution for 35-40nm silver nanocubes/DOPC monolayers deposited on different silicon films immersed in water/ethylene glycol mixtures 1/0,2/1, $1 / 2$, and $0 / 1$ volume ratios. Series correspond to the sample $A$ in Figure 5.8. Red thick curves represent experimental data. Dark blue thick curves are the result of spectral fitting. Purple lines show the difference between the fitted and experimental spectra. Blue and red peaks are quadrupolar and dipolar modes respectively.

$\mathrm{Si}, 15 \mathrm{~nm}$
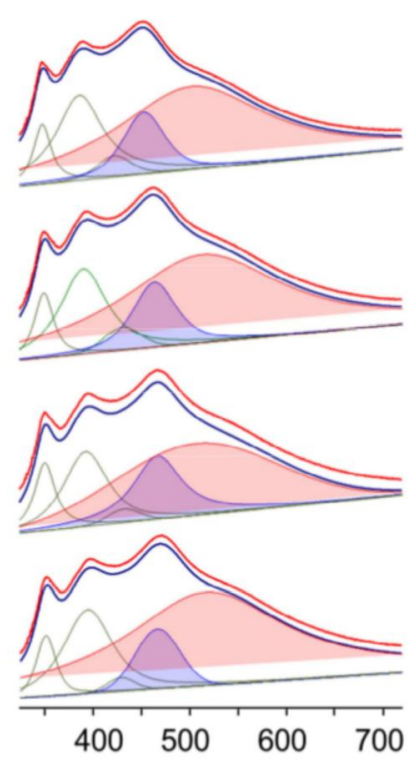

Si, $25 \mathrm{~nm}$
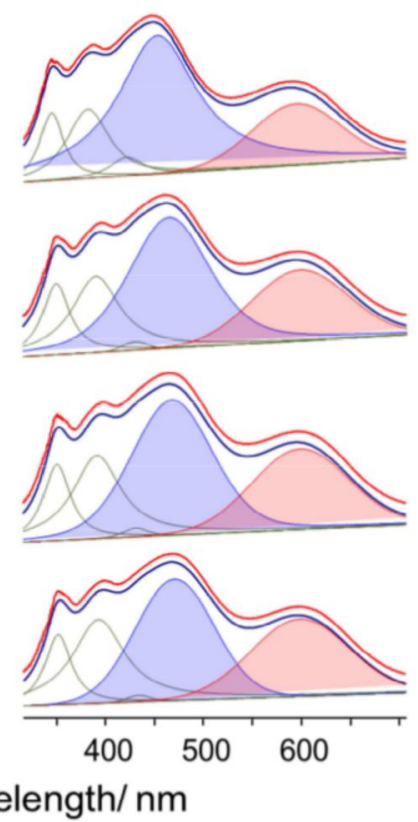

$\mathrm{H}_{2} \mathrm{O} / \mathrm{EG}$

$1 / 0$

$2 / 1$

$1 / 2$

$0 / 1$

Figure 5.10. Results of extinction spectra deconvolution for 75-80nm silver nanocubes/DOPC monolayers deposited on different silicon films immersed in water/ethylene glycol 1/0, 2/1, 1/2, and $0 / 1$ volume ratios. Series correspond to the sample $C$ in Figure 5.8. Red thick curves represent experimental data. Dark blue thick curves are the result of spectral fitting. Blue and red peaks are quadrupolar and dipolar modes respectively. 

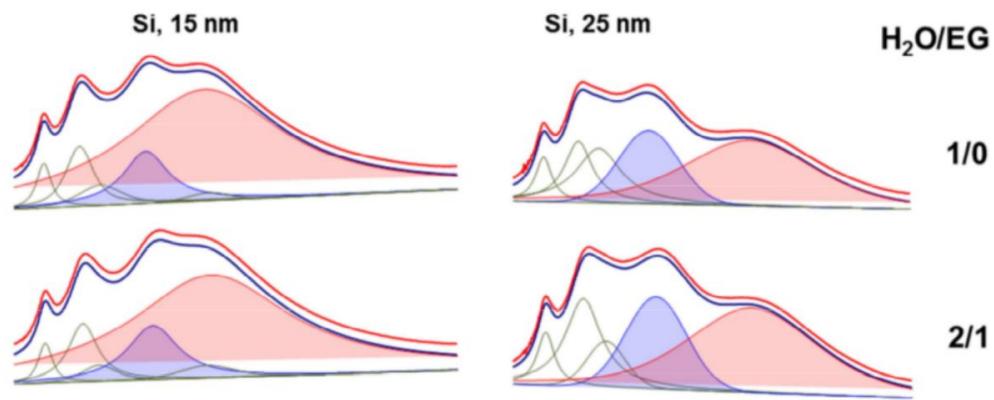

2/1
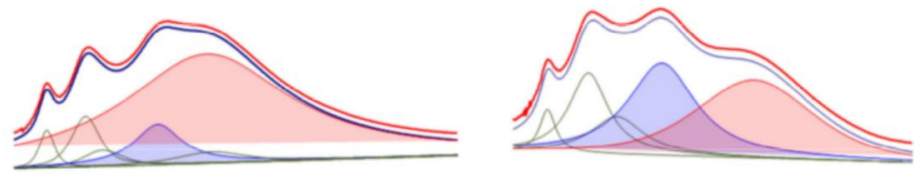

$1 / 2$
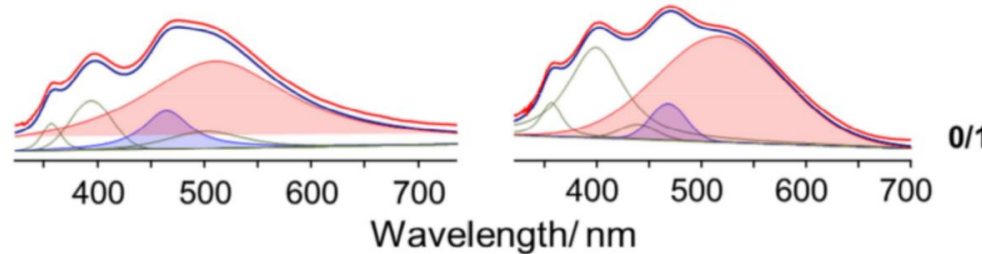

Figure 5.11. Results of extinction spectra deconvolution for $80-85 \mathrm{~nm}$ silver nanocubes/DOPC monolayers deposited on different silicon films immersed in water/ethylene glycol 1/0, 2/1, 1/2,and $0 / 1$ volume ratios. Series correspond to sample D in Figure 5.8. Red thick curves represent experimental data. Dark blue thick curves are the result of spectral fitting. Blue and red peaks are quadrupolar and dipolar modes respectively.

Table 5.1. Refractive index sensitivities of monolayers of 35-40nm silver nanocubes on silicon

\begin{tabular}{c|c|c|c}
$\begin{array}{c}\text { Silicon film } \\
\text { thickness (nm) }\end{array}$ & $\begin{array}{c}\text { Plasmon peak } \\
\text { position }(\boldsymbol{\lambda} / \mathbf{n m})\end{array}$ & $\begin{array}{c}\text { Refractive index } \\
\text { sensitivity (nm/RIU) }\end{array}$ & Figure of Merit \\
\hline \multirow{3}{*}{5} & D: 417 & $77 \pm 5.4$ & 1.38 \\
& Q: 382 & $28 \pm 8$ & 0.74 \\
350 & $44 \pm 5.3$ & 2.4 \\
\hline \multirow{2}{*}{10} & D: 427 & $42 \pm 8.1$ & 0.72 \\
& Q: 388 & $\mathbf{7 0 \pm 2 3}$ & $\mathbf{2 . 2 2}$ \\
& 350 & n.d. & n.d. \\
\hline \multirow{2}{*}{25} & D: 440 & $11 \pm 8$ & 0.18 \\
& Q: 395 & $\mathbf{7 6} \pm \mathbf{6}$ & $\mathbf{2 . 0}$ \\
& 350 & $48 \pm 0.5$ & 1.12 \\
\hline
\end{tabular}

${ }^{a}$ Not determined 
Table 5.2. Refractive index sensitivities of monolayers of $40-45 \mathrm{~nm}$ silver nanocubes on silicon

\begin{tabular}{c|c|c|c}
$\begin{array}{c}\text { Silicon film thickness } \\
(\mathbf{n m})\end{array}$ & $\begin{array}{c}\text { Plasmon peak position } \\
(\mathbf{\lambda} / \mathbf{n m})\end{array}$ & $\begin{array}{c}\text { Refractive index } \\
\text { sensitivity (nm/RIU) }\end{array}$ & Figure of Merit \\
\hline \multirow{3}{*}{5} & D: 427 & $82 \pm 3.4$ & 1.45 \\
& Q: n.d. ${ }^{a}$ & - & - \\
& 350 & $25 \pm 0.57$ & 1.64 \\
\hline \multirow{2}{*}{10} & D: 440 & $25 \pm 12$ & 0.76 \\
& Q: 407 & $\mathbf{9 5} \pm \mathbf{6 . 8}$ & 3.73 \\
& 350 & $28 \pm 1.1$ & 1.82 \\
\hline \multirow{2}{*}{25} & D: 460 & $48 \pm 4.6$ & 0.93 \\
& Q: 408 & $\mathbf{9 6} \pm \mathbf{6 . 8}$ & $\mathbf{2 . 6 7}$ \\
& 350 & $\mathbf{2 7} \pm 3.4$ & 1.60 \\
\hline
\end{tabular}

${ }^{a}$ Not determined

Table 5.3. Refractive index sensitivities of monolayers of 75-80nm silver nanocubes on silicon

\begin{tabular}{|c|c|c|c|}
\hline $\begin{array}{l}\text { Silicon film thickness } \\
\text { (nm) }\end{array}$ & $\begin{array}{c}\text { Plasmon peak position } \\
(\lambda / \mathbf{n m})\end{array}$ & $\begin{array}{c}\text { Refractive index } \\
\text { sensitivity (nm/RIU) }\end{array}$ & Figure of Merit \\
\hline 0 & $\begin{array}{c}\text { D: } 483 \\
\text { Q: } 445 \\
360 \\
340\end{array}$ & $\begin{array}{c}330 \pm 104 \\
114 \pm 15 \\
65 \pm 13 \\
26 \pm 2.7\end{array}$ & $\begin{array}{l}1.34 \\
1.57 \\
1.34 \\
1.46\end{array}$ \\
\hline 15 & $\begin{array}{c}\text { D: } 500 \\
\text { Q: } 452 \\
360 \\
340\end{array}$ & $\begin{array}{l}120 \pm 43 \\
\mathbf{1 2 5} \pm \mathbf{5 0} \\
76 \pm 9.7 \\
28 \pm 5.7\end{array}$ & $\begin{array}{c}0.6 \\
2.3 \\
1.32 \\
1.2\end{array}$ \\
\hline 25 & $\begin{array}{l}\text { D: } 600 \\
\text { Q: } 455 \\
385 \\
350\end{array}$ & $\begin{array}{c}8.2 \pm 5.6 \\
\mathbf{1 4 0} \pm \mathbf{3 7} \\
74 \pm 25 \\
13 \pm 3\end{array}$ & \begin{tabular}{c|}
0.1 \\
$\mathbf{1 . 4 4}$ \\
1.23 \\
0.41
\end{tabular} \\
\hline
\end{tabular}

${ }^{a}$ Not determined

Of particular interest is a monolayer of $80-85 \mathrm{~nm}$ cubes on a $10 \mathrm{~nm}$ silicon film (Fig. 5.13). The refractive index sensitivity for the $Q$ mode of this substrate was measured to be 237 \pm 17 nm per RIU (Fig. 5.14), which with the FWHM for that band around $36 \pm 5 n m$ results in the figure of merit of 6.6. Even though the band is fairly weak and only appears as a shoulder to the strong $\mathrm{D}$ mode in solution, the result obtained is significant as it demonstrates the highest sensitivity for the monolayer of non-interacting silver nanocubes to probe the refractive index change. Further optimization in nanocube spacing and 
monodispersity can likely improve the FOM even more. Alternative data processing procedures such as an integrated response method $^{34}$ or using a figure of merit for thin layers $^{35}$ could likely help in optimizing the system as well.

Table 5.4. Refractive index sensitivities of monolayers of 75-80nm silver nanocubes on silicon

\begin{tabular}{c|c|c|c}
$\begin{array}{c}\text { Silicon film thickness } \\
(\mathbf{n m})\end{array}$ & $\begin{array}{c}\text { Plasmon peak position } \\
(\mathbf{\lambda} / \mathbf{n m})\end{array}$ & $\begin{array}{c}\text { Refractive index } \\
\text { sensitivity (nm/RIU) }\end{array}$ & Figure of Merit \\
\hline \multirow{4}{*}{0} & D: 487 & $480 \pm 88$ & 3.0 \\
& Q: 445 & $174 \pm 6$ & 2.4 \\
& 382 & $94 \pm 42$ & $\mathbf{4 . 4}$ \\
& 350 & $18 \pm 7.8$ & 1.1 \\
\hline \multirow{4}{*}{10} & D: 494 & $64 \pm 1$ & 0.38 \\
& Q: 446 & $\mathbf{2 3 7 \pm 1 7}$ & $\mathbf{6 . 6}$ \\
& 385 & $113 \pm 7.4$ & 3.0 \\
& 350 & $58 \pm 1.7$ & 3.1 \\
\hline \multirow{4}{*}{15} & D: 504 & $49 \pm 9$ & 0.28 \\
& Q: 445 & $\mathbf{1 7 4} \pm \mathbf{1 0}$ & $\mathbf{3 . 3}$ \\
& 383 & $93 \pm 7$ & 2.65 \\
& 350 & $57 \pm 9$ & 3.2 \\
\hline \multirow{4}{*}{25} & D: 502 & $35 \pm 22$ & 0.6 \\
& Q: 443 & $\mathbf{1 2 4} \pm \mathbf{6}$ & $\mathbf{3 . 4 6}$ \\
& 383 & $69 \pm 5$ & 2.0 \\
& 350 & $39 \pm 1$ & 2.6 \\
\hline
\end{tabular}

${ }^{a}$ Not determined

Although the highest sensitivity values were obtained for the biggest cubes used in the study, we believe an optimum size exists in the 50-60nm range that should show an even greater FOM. This hypothesis is based on the dependencies of RIS and FWHM on the nanocube size: RIS increases monotonically; FWHM, on the other hand, shows a qualitative increase when the cube size exceeds $50-60 \mathrm{~nm}$ size. At this size the Q mode appears explicitly in the solution spectra ${ }^{28,29}$ and light scattering becomes the dominant contributor to extinction, leading to band broadening. ${ }^{36}$ 

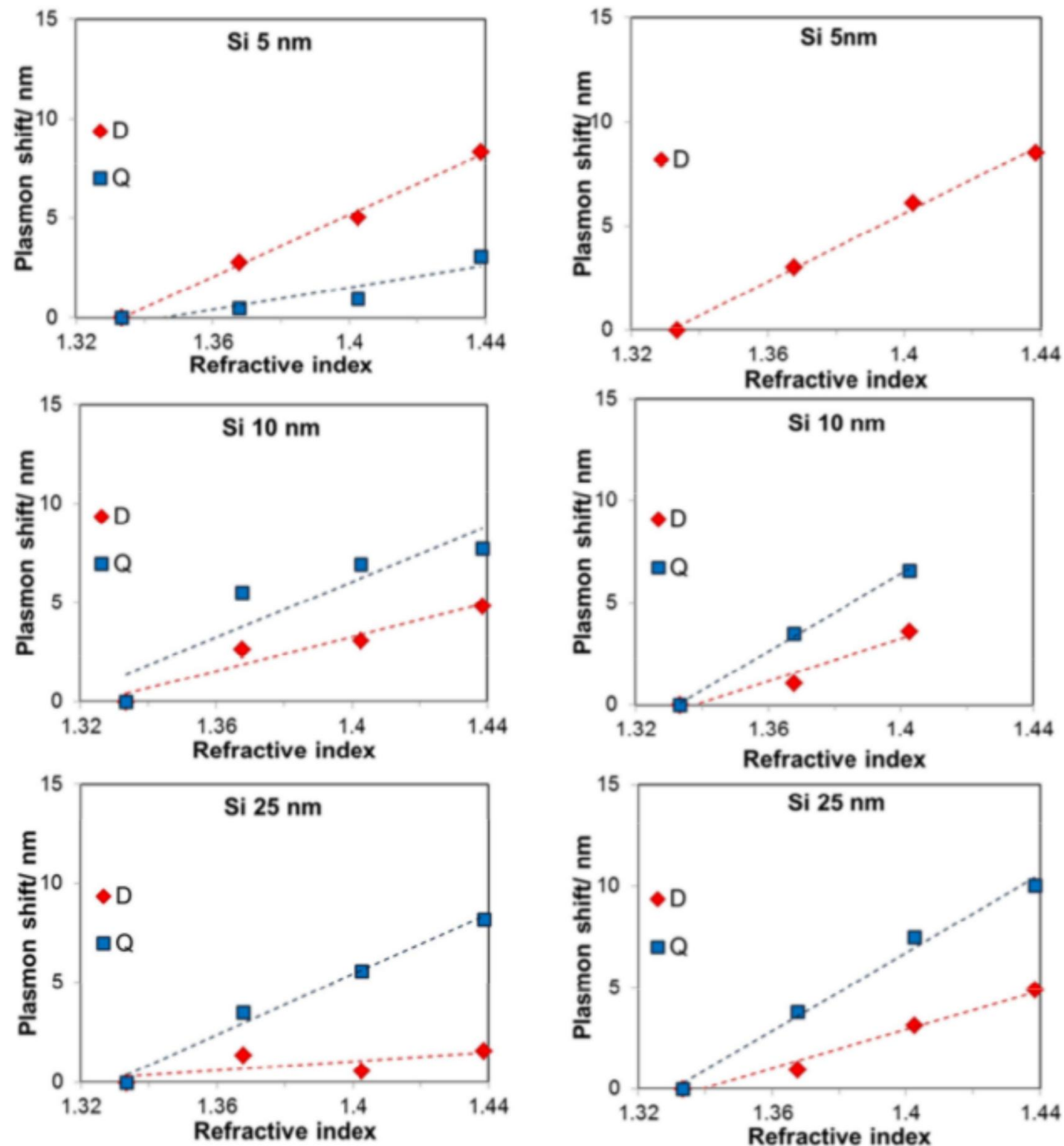

Figure 5.12A. Plasmonic shifts as functions of solution refractive index for supported nanocube/DOPC monolayers in figure $5.8 \mathrm{~A}$ (left) and B (right) 

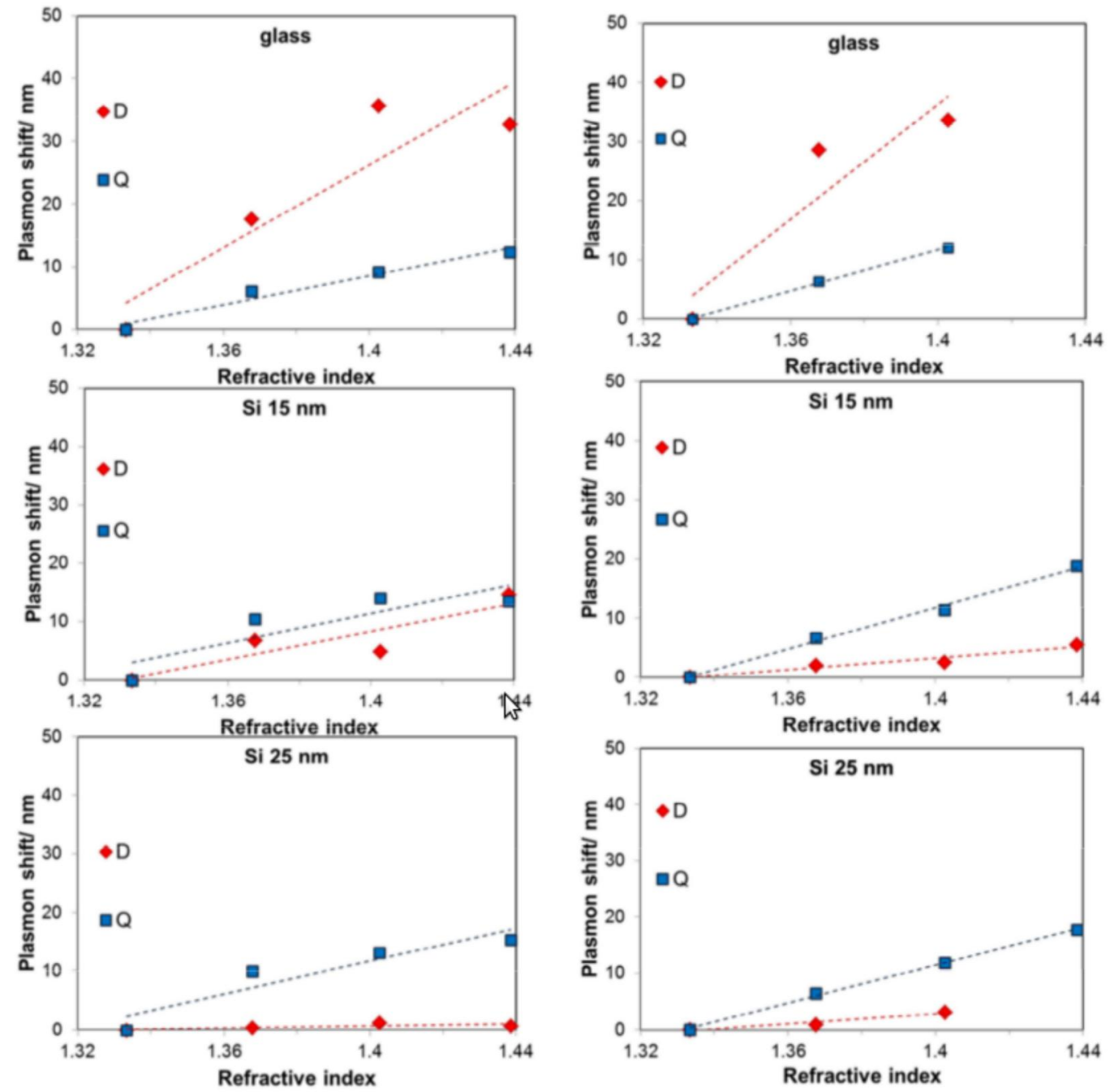

Figure 5.12B. Plasmonic shifts as functions of solution refractive index for supported nanocube/DOPC monolayers in figure $5.8 \mathrm{C}$ (left) and $\mathrm{D}$ (right). 


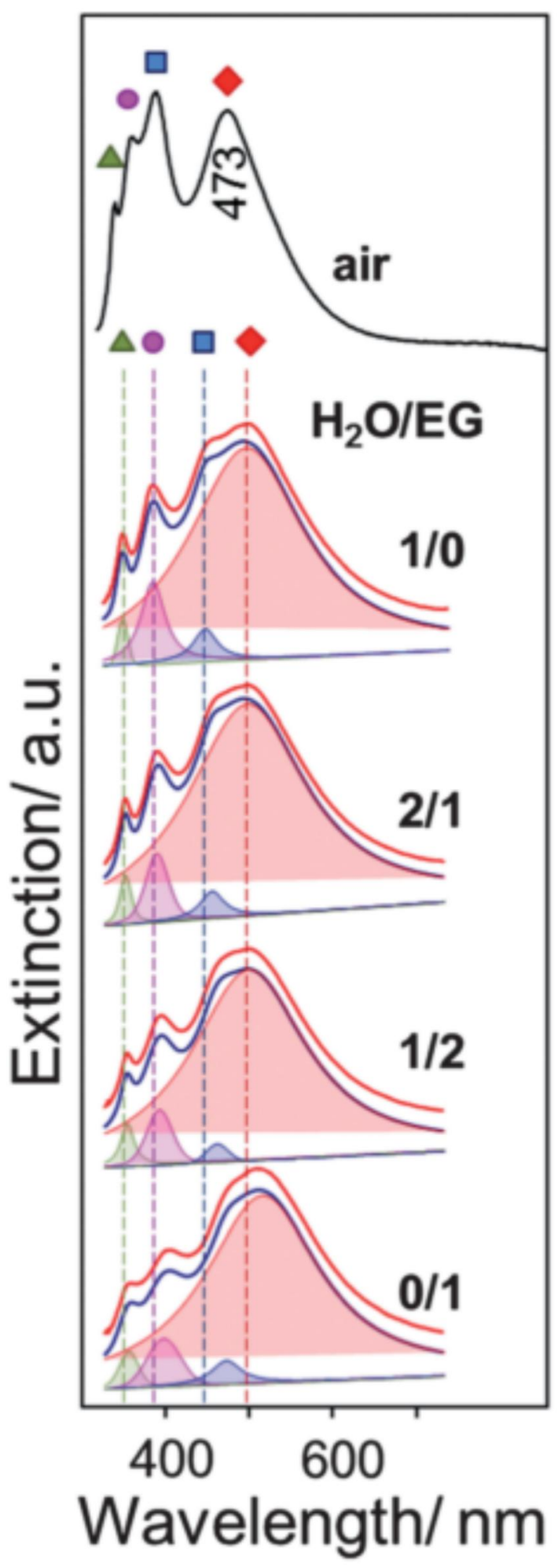

Figure 5.13. Deconvolution of extinction spectra for the $80-85 \mathrm{~nm}$ nanocube sample deposited on a 10nm silicon film in water-ethylene glycol 1/0, 2/1, 1/2, and 0/1 mixtures. Red thick curves represent experimental data. Dark blue thick curves are the result of spectral fitting. Blue and red peaks are quadrupolar and dipolar modes respectively. The top black spectrum is measured in air. 


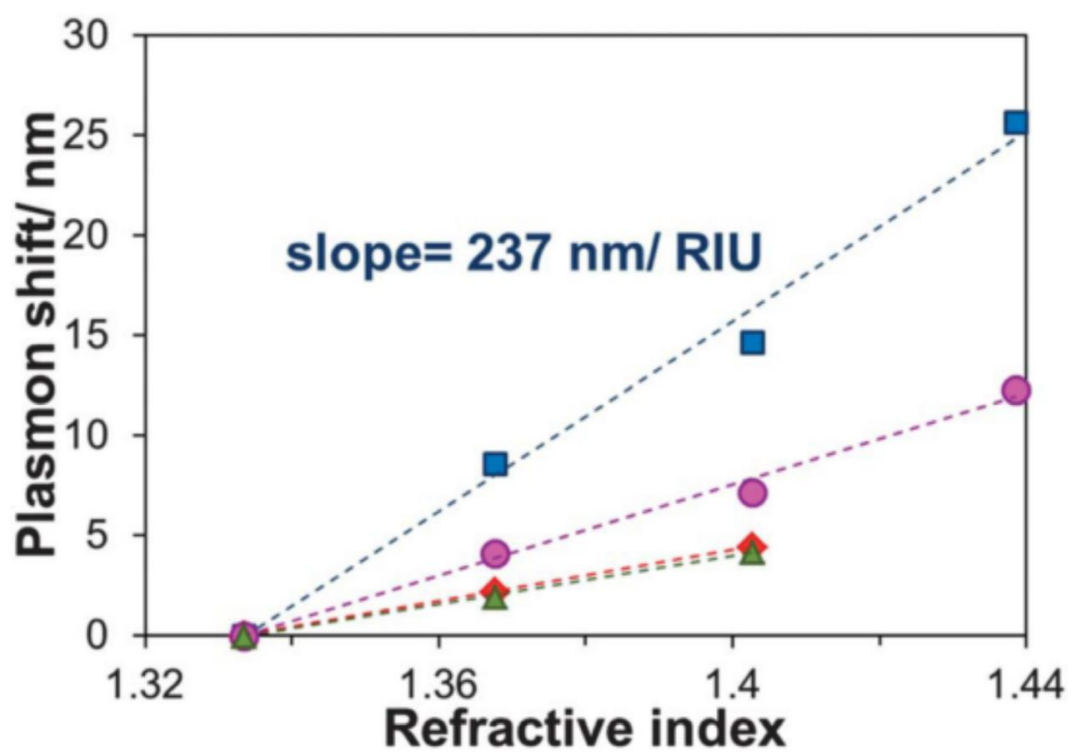

Figure 5.14. Plasmonic shifts as functions of refractive index for supported nanocube-DOPC monolayers for peaks shown in Fig. 5.13.

\section{Dipolar band shifting enables stronger Raman signal enhancement:}

The ability to fine tune the hybrid plasmon modes can be utilized for investigating and optimizing phenomena that rely on the increase of electric field strength near the surface of metal nanoparticles such as surface enhanced Raman scattering (SERS) or fluorescence. Since the D mode of the supported nanocube monolayer is very sensitive to the substrate refractive index, by increasing the supporting silicon film thickness the mode can be effectively tuned to coincide with the desired Raman or fluorescence excitation wavelength for effective field enhancement by the D LSPR. For example, Fig. 5.15 shows UV-vis extinction spectra for a monolayer of weakly interacting $\sim 60 \mathrm{~nm}$ nanocubes on glass (dotted blue line) and on a 7nm silicon film (dotted red line). The dipolar mode of the silicon supported film appears around 460nm and, unlike the glass supported monolayer dipolar band, coincides conveniently with the $458 \mathrm{~nm}$ of the argon ion laser. As a result stronger enhancement of the Raman signal was observed for the silicon versus glass supported nanocubes when using this wavelength. The SERS signal intensity of the rhodamine 6G 
(Rh6G) sample was found to be 10 times greater on silicon (Fig. 5.15 and 5.16) when excited with 458nm wavelength.

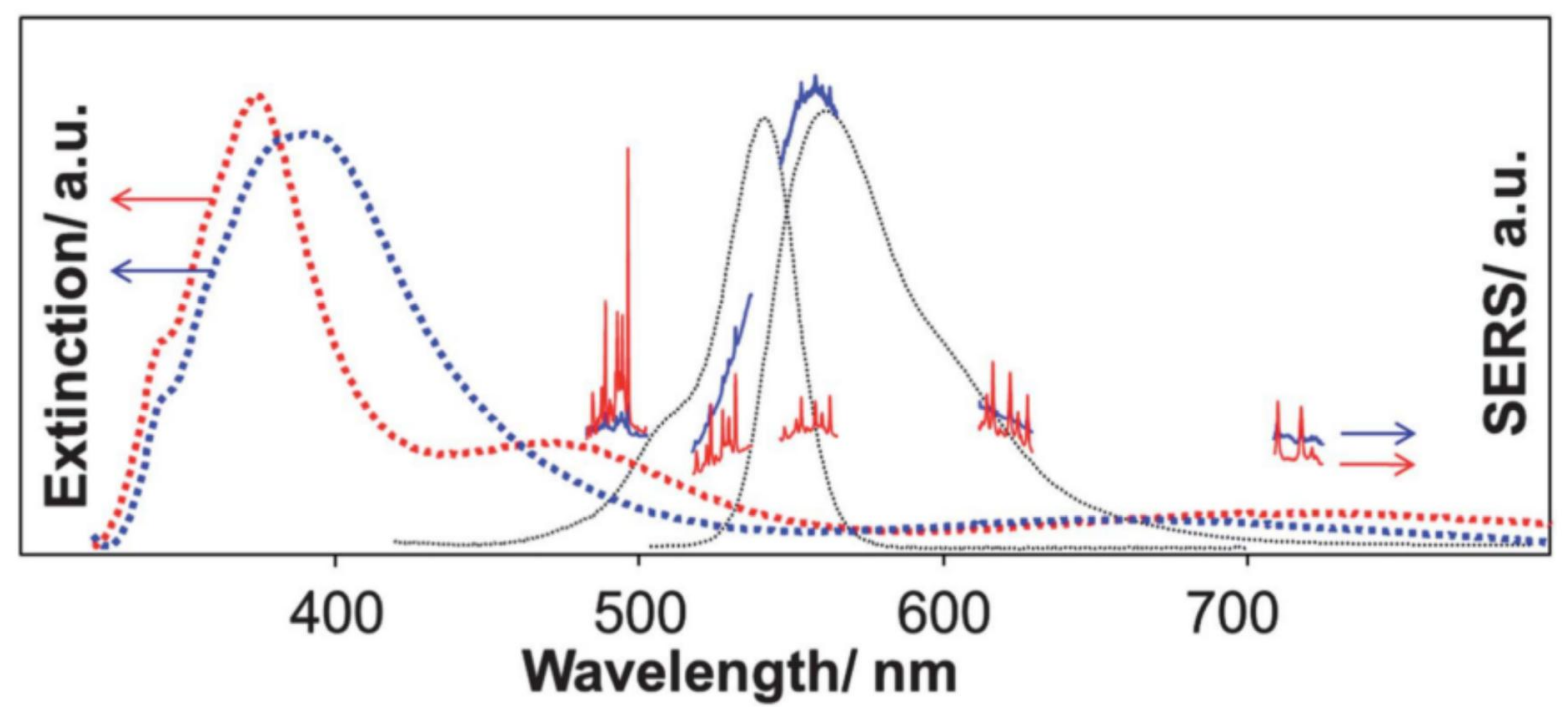

Figure 5.15. SERS spectra of rhodamine 6G on glass (blue curves) and a $7 \mathrm{~nm}$ silicon film (red curves) at different excitation wavelengths 458, 488, 514, 568 and 647nm. Extinction spectra for nanocube-lipid monolayers on glass (blue dashed curve) and silicon (red dashed curve) are presented. Extinction and fluorescence spectra for Rh6G are shown as black dotted lines. SERS spectra were normalized by using the intensity of the $1508 \mathrm{~cm}^{-1}$ band of Rh6G on glass at different wavelengths.

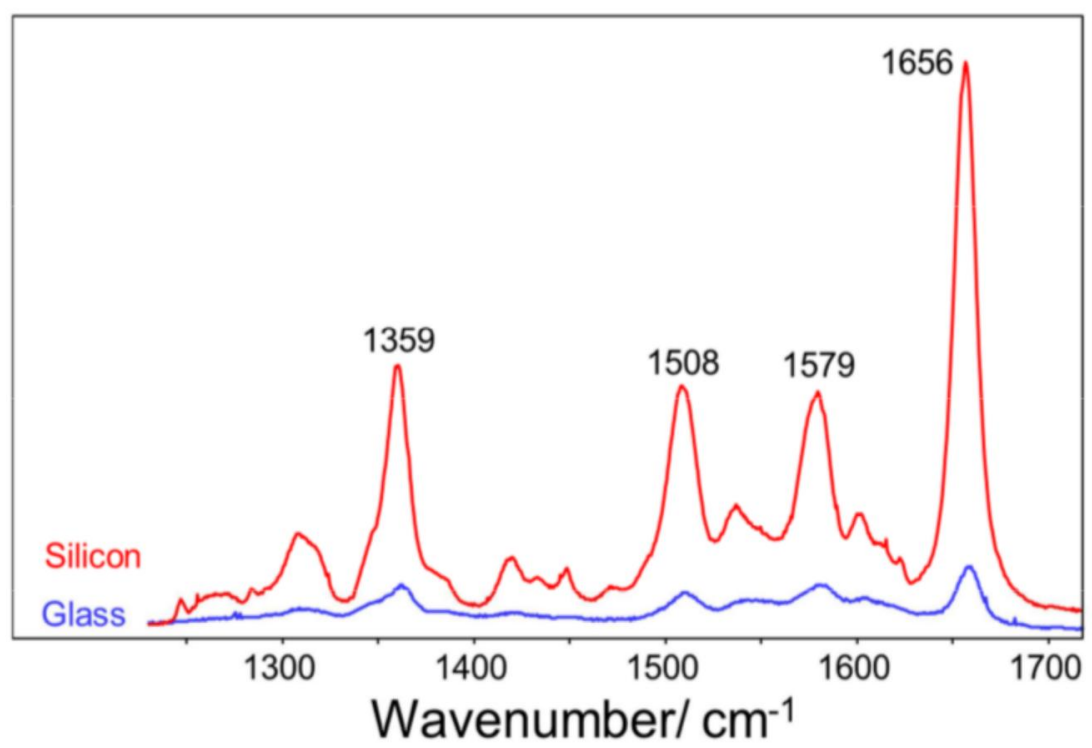

Figure 5.16. SERS spectra of Rhodamine $6 \mathrm{G}(1 \mu \mathrm{M})$ deposited on a monolayer of $\sim 60 \mathrm{~nm}$ silver nanocubes/DOPC on glass (blue) and $7 \mathrm{~nm}$ silicon film (red). The spectra were obtained by averaging spectra from 9 different areas of the sample. Accumulation time in each point was 60 seconds. Excitation wavelength used was $458 \mathrm{~nm}$ with laser power below $1 \mathrm{~mW}$. 
When other excitation wavelengths were used the difference in the signal intensity was not as significant (Fig. 5.15, SERS spectra), however, silicon supported nanocubes provided a stronger enhancement in the entire visible region, as can be seen from the Fig. 5.15 spectra and Fig. 5.17 enhancement factors.

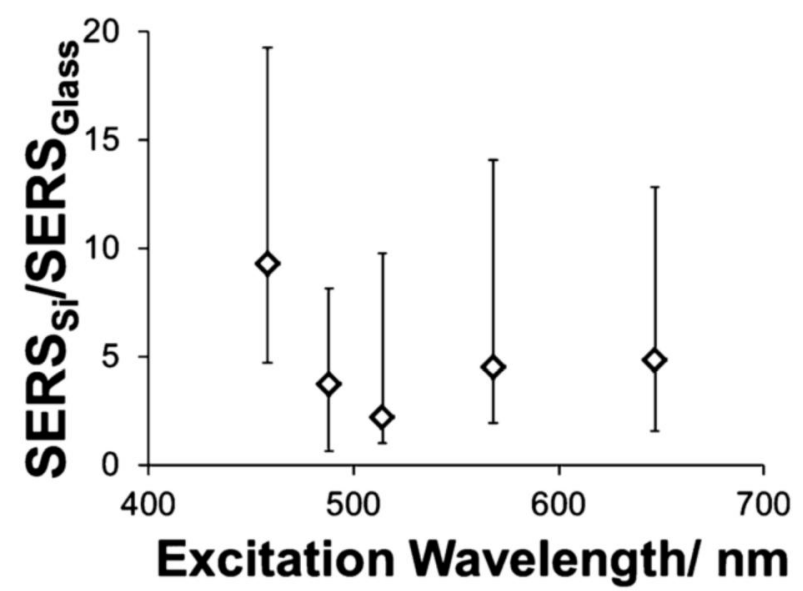

Fig. 5.17 SERS enhancement factor as a function of excitation wavelength for spectra shown in Fig. 5.15.

However, not only the Raman signal intensity was modified when silicon supported nanocubes were used. When comparing the fluorescence signal background for Rh6G on the two substrates it was found to be 10 times stronger on glass than on silicon (Fig. 5.15). Yet, when the laser power was increased to 5, 25, or 50mW a decrease in SERS or fluorescence signals with time could be detected accompanied by amorphous carbon formation (Fig. 5.18 and 5.19) as a result of Rh6G photochemical degradation. ${ }^{37-40}$ The process took place at both silicon and glass parts of the substrate, but was considerably (2-10 times) faster on the glass side. Since the nanocube monolayer was deposited simultaneously on the glass and silicon sides of the substrate and Rh6G adsorption was done simultaneously as well the effect does not result from the difference in nanoparticle density or Rh6G concentration. Therefore stronger fluorescence quenching and decreased rates of amorphous carbon formation clearly indicate the existence of additional de-excitation pathways for Rh6G on silicon compared to 
the glass supported nanocube monolayer. As a result the molecules do not reside in the excited state long enough to undergo photocatalytic degradation.

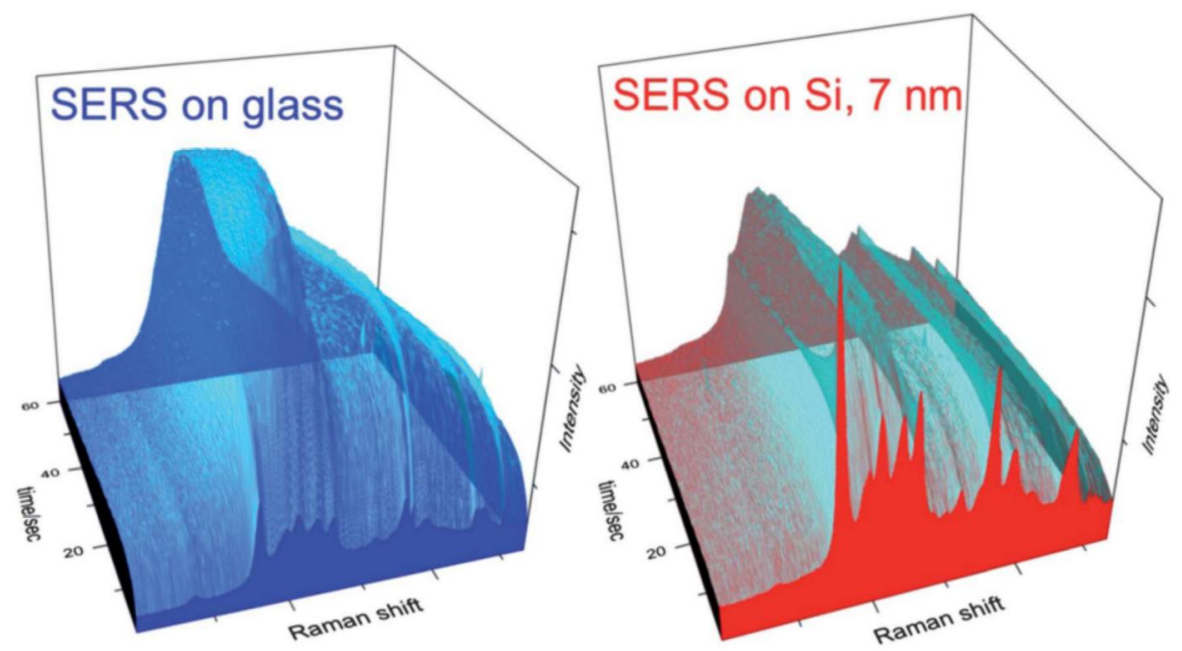

Figure 5.18. Time dependence of the rhodamine 6G SERS spectrum on glass (blue) and a $7 \mathrm{~nm}$ silicon film (red). The excitation wavelength was $488 \mathrm{~nm}$ and power was $50 \mathrm{~mW}$. Disappearance of the SERS spectrum and the appearance of the carbon spectrum occur faster on glass.

There are several possible mechanisms of de-excitation: ${ }^{41}$ non-radiative dipoledipole interaction that has been observed and well described at a semiconductor surface; ${ }^{42,43}$ excitation of waveguide modes; ${ }^{44,45}$ and excitation of dipolar LSPR. ${ }^{46}$ The first mechanism predicts faster de-excitation on silicon due to the stronger image-dipole as compared to glass. The second mechanism should only be present on silicon since the waveguide modes only exist in thin silicon films. Finally, the third mechanism also predicts stronger deexcitation on a silicon substrate due to the greater overlap between the Rh6G fluorescence and D LSPR. In that respect not only can silicon substrates serve the purpose of shifting the dipolar mode to the desired spectral range for optimization of the Raman signal enhancement but also create a favourable platform for molecular stability, preventing photochemical degradation. It is likely that a thin silica film at the silicon surface is present in our experiments. This should not, however, change the observations qualitatively and was confirmed by the experiments with hydrogen terminated silicon substrates. 

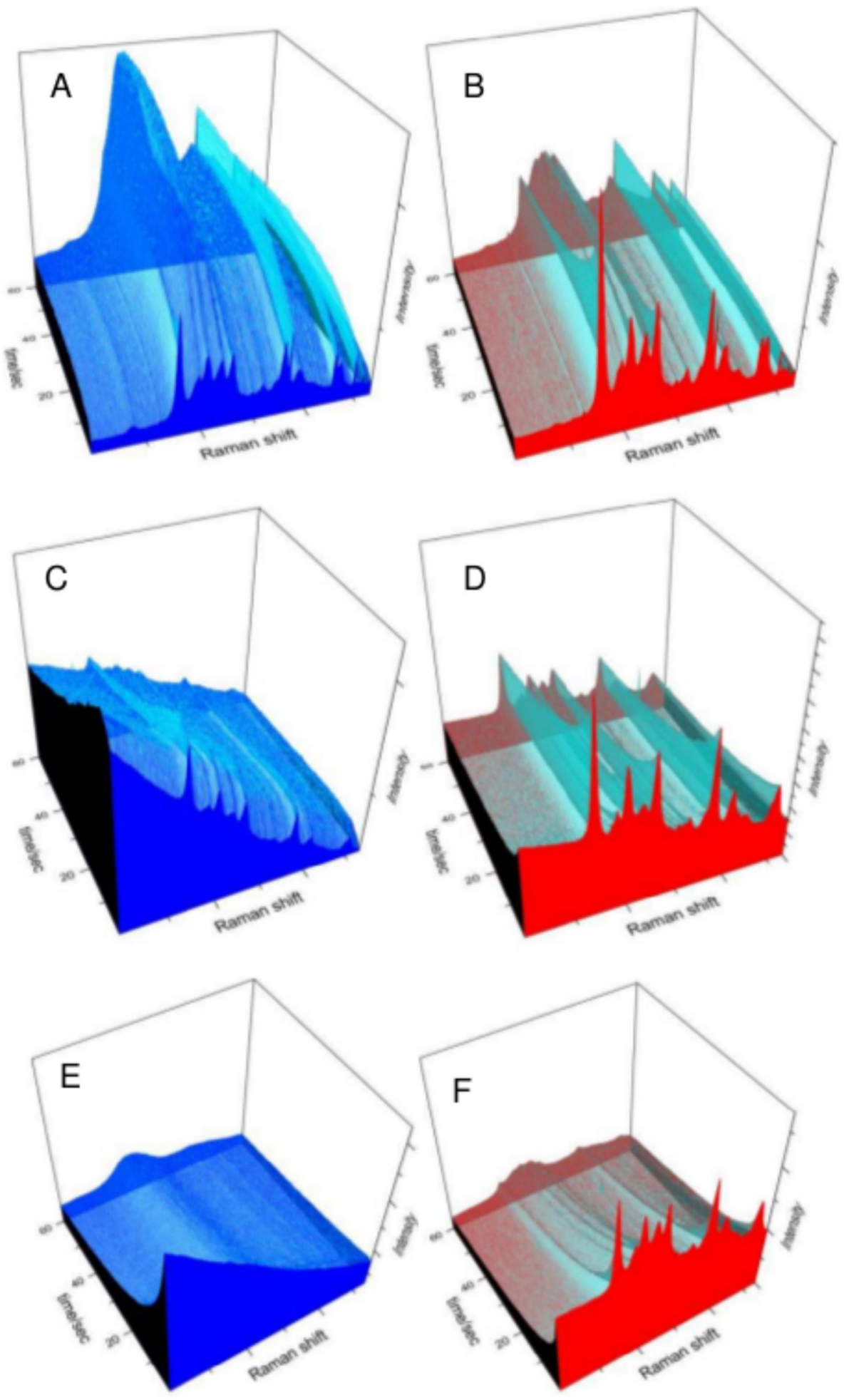

Figure 5.19. SERS spectra of Rhodamine $6 \mathrm{G}(1 \mu \mathrm{M})$ deposited on a monolayer of $\sim 60 \mathrm{~nm}$ silver nanocubes/DOPC on a glass ( $A, C, E$, blue surfaces) and $7 \mathrm{~nm}$ silicon film (B,D,F, red surfaces). Time progression of the spectra over 60 seconds is presented. Excitation wavelength of $458(A, B)$ or 488 $(C-F) n m$ was used. Laser power was $25 \mathrm{~mW}(A, B) ; 5 \mathrm{~mW}(C, D)$, and $50 \mathrm{~mW}(E, F)$. 


\subsection{Conclusions}

In conclusion, we demonstrated a simple but effective way to control plasmonic properties of weakly interacting silver nanocubes by supporting them on thin silicon films. A fine relationship between such films thickness and plasmonic properties was observed. Silicon films with a thickness gradient will likely be very useful in consequent studies.

\subsection{References}

1 Y. Sun and Y. Xia, Science, 2002, 298, 2176-2179.

2 L. J. Sherry, S.-H. Chang, G. C. Schatz, R. P. Van Duyne, B. J. Wiley and Y. Xia, Nano Lett., 2005, 5, 2034-2038.

3 J. M. McMahon, Y. Wang, L. J. Sherry, R. P. Van Duyne, L. D. Marks, S. K. Gray and G. C. Schatz, J. Phys. Chem. C, 2009, 113, 2731-2735.

4 E. Ringe, J. M. McMahon, K. Sohn, C. Cobley, Y. Xia, J. Huang, G. C. Schatz, L. D. Marks and R. P. Van Duyne, J. Phys. Chem. C, 2010, 114, 12511-12516.

5 Y. H. Lee, H. Chen, Q.-H. Xu and J. Wang, J. Phys. Chem. C, 2011, 115, 7997-8004.

$6 \quad$ N. Ahamad and A. Ianoul, J. Phys. Chem. C, 2011, 115, 3587-3594.

$7 \quad$ M.A.Mahmoud, C. E. Tabor and M. A. El-Sayed, J. Phys. Chem. C, 2009, 113, 54935501.

8 M. A. Mahmoud and M. A. El-Sayed, J. Phys. Chem. C, 2008, 112, 14618-14625.

9 A. R. Tao, J. Huang and P. Yang, Acc. Chem. Res., 2008, 41, 1662- 1673.

10 S. Y. Lee, L. Hung, G. S. Lang, J. F. Cornett, I. D. Mayergoyz and O. Rabin, ACS Nano, 2010, 4, 5763-5772.

11 M. Rycenga, P. H. Camargo,W. Li, C. H. Moran and Y. Xia, J. Phys. Chem. Lett., 2010, 1, 696-703.

12 W. J. Galush, S. A. Shelby, M. J. Mulvihill, A. Tao, P. Yang and J. T. Groves, Nano Lett., 2009, 9, 2077-2082.

13 M. K. Knight, Y. Wu, J. B. Lassiter, P. Nordlander and N. J. Halas, Nano Lett., 2009, 9, 2188-2192.

14 P. Albella, B. Garcia-Cuerto, F. Gonzalez, F. Moreno, P. C. Wu, T.-H. Kim, A. Brown, 
Y. Yang, H. O. Everitt and G. Videen, Nano Lett., 2011, 11, 3531-3537.

15 P. A. Letnes, I. Simonen and D. L. Mills, Phys. Rev. B: Condens. Matter Mater. Phys., 2011, 83, 075426.

16 M. D. Malinsky, K. L. Kelly, G. C. Schatz and R. P. Van Duyne, J. Phys. Chem. B, 2001, 105, 2343-2350.

17 N. Pinna, M. Maillard, A. Courty, V. Russier and M. P. Pileni, Phys. Rev. B: Condens. Matter Mater. Phys., 2002, 66, 045415.

18 M. A. Mahmoud, M. Chamanzar, A. Adibi and M. A. El-Sayed, J. Am. Chem. Soc., 2012, 134, 6434-6442.

19 H. Chen, L. Shao, T. Ming, K. C. Woo, Y. C. Man, J. Wang and H.-Q. Lin, ACS Nano, 2011, 5, 6754-6763.

20 F. J. Beck, E. Verhagen, S. M. Mokkapati, A. Polman and K. R. Catchpole, Opt. Express, 2011, 19, A146-A156.

21 S. Zhang, K. Bao, N. J. Halas, H. Xu and P. Nordlander, Nano Lett., 2011, 11, 16571663.

22 N. Ahamad, A. Bottomley and A. Ianoul, J. Phys. Chem. C, 2012, 116, 185-192.

23 H. Chen, T. Ming, S. Zhang, Z. Jin, B. Yang and J. Wang, ACS Nano, 2011, 5, 48654877.

24 K. R. Catchpole and A. Polman, Appl. Phys. Lett., 2008, 93, 191113.

25 S. Pillai, K. R. Catchpole, T. Trupke and M. A. Green, J. Appl. Phys., 2007, 101, 093105.

26 A. Bottomley and A. Ianoul, ChemPhysChem, 2011, 12, 2912-2914.

27 N. Ahamad, D. Prezgot and A. Ianoul, J. Nanopart. Res., 2012, 14, 724.

28 X. Xia, J. Zeng, L. K. Oetjen, Q. Liand and Y. Xia, J. Am. Chem. Soc., 2012, 134, 1793-1801.

29 F. Zhou, Z.-Y. Li, Y. Liu and Y. Xia, J. Phys. Chem. C, 2008, 112, 20233-20240.

30 A. V. Whitney, J. W. Elam, S. Zou, A. V. Zinovev, P. C. Stair, G. C. Schatz and R. P. Van Duyne, J. Phys. Chem. B, 2005, 109, 20522-20528.

31 D. D. Evanoff, R. L. White and G. Chumanov, J. Phys. Chem. B, 2004, 108, 1522 1524. 
32 F. Hao, Y. Sonnefraud, P. Van Dorpe, S. A. Maier, N. J. Halas and P. Nordlander, Nano Lett., 2008, 8, 3983-3988.

33 A. Feofanov, A. Ianoul, E. Kryukov, S. Maskevich, G. Vasiliuk, L. Kivach and I. Nabiev, Anal. Chem., 1997, 69, 3731-3740.

34 M. Das, D. Hohertz, R. Nirwan, A. G. Brolo, K. L. Kavanagh and R. Gordon, IEEE Photonics J., 2011, 3, 441-449.

35 J. Becker, A. trugler, A. Jakab, U. Hohenester and C. Sonnichsen, Plasmonics, 2010, 5, 161-167.

36 D. D. Evanoff and G. Chumanov, ChemPhysChem, 2005, 6, 1-12.

37 J. I. Gersten and A. Nitzan, Surf. Sci., 1985, 158, 165-189.

38 E. J. Bjerneld, F. Svedberg, P. Johansson and M. Kall, J. Phys. Chem. A, 2004, 108, 4187-4193.

39 J. S. Suh, M. Moskovits and J. Shakhesemampour, J. Phys. Chem., 1993, 97, 16781683.

40 R. A. Alvarez-Puebla, J. Phys. Chem. Lett., 2012, 3, 857-866.

41 W. L. Barnes, J. Mod. Phys., 1998, 45, 661-699.

42 T. Hayashi, T. G. Castner and R. W. Boyd, Chem. Phys. Lett., 1983, 94, 461-466.

43 P. M. Whitemore, A. P. Alivisatos and C. B. Harris, Phys. Rev. Lett., 1983, 50, 10921094.

44 H. R. Stuart and D. G. Hall, Phys. Rev. Lett., 1998, 80, 5663- 5666.

45 B. J. Soller and D. G. Hall, J. Opt. Soc. Am. A, 2001, 18, 2577- 2584.

46 M. Thomas, J.-J. Greffet, R. Carminati and J. R. Arias-Gonzalez, Appl. Phys. Lett., 2004, 85, 3863-3865. 
Chapter 6

Reflection and Absorption Spectra of Silver Nanocubes on a Dielectric Substrate:

Anisotropy, Angle, and Polarization Dependencies

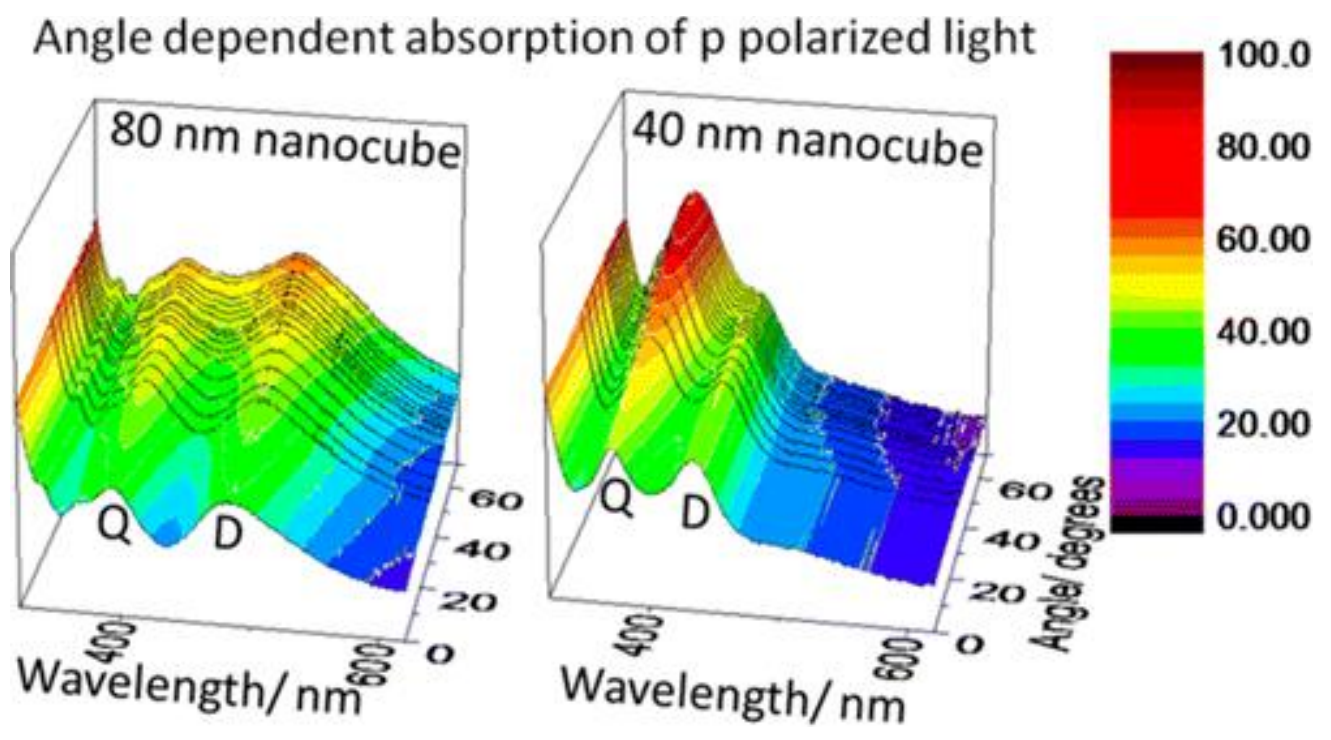


Modified from original manuscript published as:

Bottomley, A. and Ianoul, A., Reflection and Absorption Spectra of Silver Nanocubes on a Dielectric Substrate: Anisotropy, Angle, and Polarization Dependencies. J. Phys. Chem. C 2014, 27509-27515.

doi:10.1021/jp508629d

Department of Chemistry, Carleton University, 1125 Colonel By Drive, Ottawa, Ontario, Canada, K1S 5B6

\subsection{Abstract}

Angle and polarization dependent UV-visible reflection and transmission spectra were measured for monolayers of weakly interacting silver nanocubes (of 40 and 80nm edge lengths) supported by thin films of titanium oxide on glass utilizing both front and rear geometries with respect to the incident radiation. The supporting substrate mediates the hybridization of dipolar and quadrupolar plasmon resonances. From the spectra absolute extinction and absorption cross sections for supported silver nanocubes were determined. A strong angle dependence of the extinction and reflection spectra for p- but not s-polarized light was observed. This dependence is considerably more pronounced for the hybridized quadrupolar resonance of the 40nm cubes. When illuminated from the rear, a significantly stronger absorption of light by the dipolar but not the quadrupolar resonance occurs for both cube sizes. Such absorption anisotropy could result from the wavelength dependent constructive or destructive interference between incident and scattered light from the nanocubes. This study demonstrates the impact of a dielectric substrate on the optical properties of novel plasmonic materials.

\subsection{Introduction}

It has been recognized that properties of supported plasmonic nanoparticles differ quite significantly from those of nanoparticles suspended in a solution. ${ }^{1,2}$ The effect depends on the local refractive index as well as on certain physicochemical characteristics of the 
substrate leading to appearance of new behaviours. Such phenomena include hybridization of plasmonic modes in silver nanoparticles induced by proximity to a dielectric substrate mediated by the refractive index of the both the substrate and the superstrate (usually air). ${ }^{3-5}$ The resulting modes could interfere with each other generating the appearance of Fano like resonances in their extinction spectra. ${ }^{6,7}$ Anisotropic Raman signal enhancement by plasmonic nanoparticles is another phenomenon that derives from the dielectric substrate induced changes in light behaviour. ${ }^{8}$ Anisotropic photoelectric current in plasmonic solar cells has also been observed and is related to electron tunnelling through the substrate. ${ }^{9-12}$ Finally, the appearance of a gap mode for gold supported silver nanocubes has also been demonstrated. This gap mode appears due to excitation of surface plasmon polaritons (SPP) in the support. ${ }^{13,14}$ In all cases the nature (dielectric, semiconductor, metal) and properties (refractive index, conductivity, ability to support SPP) of the substrate determines the existence and magnitude of any particular phenomenon.

Motivated by these findings we began a systematic investigation into the effect of the supporting substrate on optical properties of supported silver nanocubes. Previously we have reported that by choosing an appropriate dielectric substrate it is possible to tune the degree of plasmon mode hybridization and improve the refractive index sensitivity and surface enhanced Raman scattering (SERS) signal. ${ }^{15}$ In this work we investigate the effect of the supporting dielectric substrate on visible light extinction by weakly interacting silver nanocubes.

In particular, we looked to investigate how electron oscillations corresponding to various hybridized resonances can be excited by varying polarization, angle, and illumination geometry (illumination from air or through the substrate). Nanocubes were chosen as they support higher order resonances at relatively small sizes compared to 
spherical geometries in addition to the large contact area the cubes make with the substrate, yielding a more pronounced effect. ${ }^{16}$ We chose nanocubes of two sizes: 40 and $80 \mathrm{~nm}$. While suspended in an isotropic environment the former only supports dipolar and octupolar resonances, whereas the latter supports additional higher order modes. When deposited on a dielectric substrate both cube sizes support hybrid dipolar and quadrupolar modes that are spatially separated in and above the substrate. Finally, we chose thin films of titanium oxide on glass as a method to vary the refractive index of the substrate without supporting SPPs and minimizing electron tunnelling through the substrate.

\subsection{Materials and Methods}

Chemicals: Silver nitrate $\left(\mathrm{AgNO}_{3}, 99+\%\right)$, polyvinylpyrrolidone (PVP, $\mathrm{M}_{\mathrm{w}} \sim 55$ 000), anhydrous $99.8 \%$ ethylene glycol (EG), sodium sulfide, and chloroform were purchased from Sigma-Aldrich and used as obtained. Ethanol (95\%) was obtained from Commercial Alcohols and used without further purification. 1,2-Dioleoyl-sn-glycero-3phosphocholine (DOPC) was purchased from Avanti Polar Lipids as a powder and used as provided.

Preparation of Nanocubes: Nanocubes were synthesized according to the procedure described in the literature. ${ }^{17}$ Typically, a $100 \mathrm{~mL}$ round-bottom flask containing $35 \mathrm{~mL}$ of EG and a clean stir bar was placed in an oil bath set to $150^{\circ} \mathrm{C}$ and allowed to heat for $1 \mathrm{~h}$. Using a glass syringe $5 \mathrm{~mL}$ of EG containing $0.4 \mathrm{~g}$ of dissolved PVP was injected. Five minutes later using a micropipette, $400 \mu \mathrm{L}$ of $3 \mathrm{mM}$ sodium sulfide dissolved in EG was added. After an additional $5 \mathrm{~min}$, 2.5mL of 282mM silver nitrate dissolved in EG was injected slowly using a glass syringe. Upon addition of the silver nitrate the solution immediately turned black and slowly became a transparent yellow, then changed to an ochre colour while some plating in the flask occurred. The reaction was monitored by periodically taking small aliquots out of 
the reaction flask using a Pasteur pipet and dispersing it in a cuvette filled with ethanol for UV-visible spectroscopy. To yield smaller 40nm cubes the reaction was quenched when the appearance of a small but distinct peak at 350nm was observed in the spectrum. To obtain larger cubes with an edge length of $80 \mathrm{~nm}$ an additional $7 \mathrm{~mL}$ of $282 \mathrm{mM}$ silver nitrate in EG was added quickly to the reaction at this time and monitored until the desired spectrum was observed and the reaction quenched.

Purification of Colloidal Silver Nanocubes: The as synthesized colloidal nanocubes begin suspended in EG, containing excess PVP and nanorods as a reaction by product. EG and PVP were removed by diluting the colloidal solution with ethanol (1:1 by volume) followed by centrifugation at $13800 \mathrm{~g}$. The precipitate was collected and redispersed in ethanol via sonication. This process was repeated multiple times for complete removal of EG and PVP. Silver nanorods were separated from the nanocubes by filtering the prewashed NCs in ethanol using PTFE syringe filters (pore size: 0.45 and $0.22 \mu \mathrm{m}$ )

Preparation of LB Monolayers: Nanocube substrates were prepared according to the procedure reported previously. ${ }^{16}$ Briefly, NIMA 311D trough, filled with Milli-Q water (18.2 MScm) was used to prepare the Langmuir films of different NC samples. In order to form monolayers an appropriate amount of each sample was transferred into chloroform and deposited onto the surface of the water using a microsyringe. Each monolayer was left for 20min to allow complete chloroform evaporation. The surface pressure of the monolayer was measured with a paper Wilhelmy plate. Before transferring the monolayer onto solid supports several isotherm cycles were performed to anneal the films. Monolayers of nanocube/lipid mixtures were transferred onto clean substrates at pressures corresponding to the gas phase of the isotherm and with a dip rate of $2 \mathrm{~mm} / \mathrm{min}$ at room temperature.

UV-vis Measurements: UV-vis spectra of the colloidal nanocubes in ethanol and of 
monolayers deposited on the substrates were recorded using Ocean Optics 2000 optic fiber spectrometer and xenon pulse lamp. Polarization was controlled with an ultra broadband wire grid linear polarizer (Edmund Optics). Measurements were performed in front (Figure 6.1 left) and rear (Figure 6.1 right) illumination. Substrates were recorded using Ocean Optics 2000 optic fiber spectrometer and xenon pulse lamp. Polarization was controlled with an ultra broadband wire grid linear polarizer (Edmund Optics). Measurements were performed in front (Figure 1 left) and rear (Figure 1 right) illumination geometries. Irregularities in particle distribution and/or the substrate can influence the peak widths and positions observed in the extinction spectrum, so a position corresponding the most occurring spectrum or statistical mode was chosen as the initial spectra and kept in place for a whole series. A home-built goniometer was used to control the angle of incidence and collect the appropriate reflection and transmission signals. All the measurements were performed with respect to unattenuated light which was used as $100 \%$ intensity $I_{0}$. To calculate the extinction or absorption cross sections the following relationship was used:

$$
I=I_{0} 10^{-\varepsilon \sigma}
$$

where I is the intensity of light lost at the sample, $\varepsilon$ ( $\mathrm{m}^{2} /$ nanocube) is the cross section of interest, and $\sigma$ (nanocubes $\left./ \mathrm{m}^{2}\right)$ is the nanocube surface density in a given monolayer.

$$
\begin{aligned}
& \text { total extinction }=100-T \\
& \text { absorption }=100-T-R
\end{aligned}
$$

It is important to note that the absorption term calculated in eq. 6.3 includes absorption, scattering, and intensity lost to the substrate acting as a waveguide. Spectra were analyzed using Origin Laboratories and GRAMS/AI spectral data processing software. 


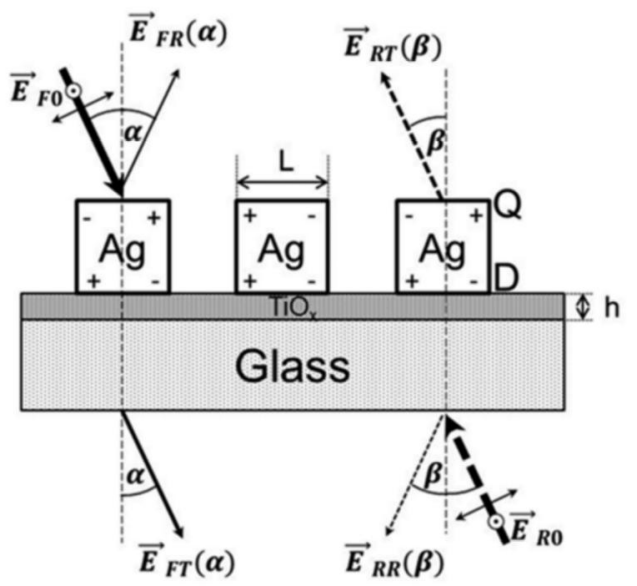

Figure 6.1. Experimental scheme: silver nanocubes of 40 and $80 \mathrm{~nm}$ average edge length were deposited by the Langmuir-Blodgett method onto a glass substrate containing a thin titanium oxide film of 0,5 , and 20nm thickness. Reflection (R) and transmission ( $T$ ) spectra were measured for $\mathrm{s}-$ and $\mathrm{p}$-polarization for front (left) or rear (right) illumination geometry. Absorption was calculated as $100-T-R$. Interparticle separation was controlled to minimize coupling.

Topography Measurements: The topography of the NC monolayer, transferred onto glass substrates at different surface pressures, was obtained using an Ntegra (NTMDT, Russia) atomic force microscope in semi contact mode in air at $21^{\circ} \mathrm{C}$ with $512 \times 512$ points per image. A $100 \times 100 \mu \mathrm{m}^{2}$ scanner (Ntegra) and cantilevers with rotated monolithic silicon tips (135 $\mu \mathrm{m}$ long, $0.3-6 \mathrm{~N} / \mathrm{m}$ spring constant NSG03, resonance frequency $90 \mathrm{kHz}, \mathrm{NT}$ MDT) were used for all topographic measurements. The typical scan rate was $0.5 \mathrm{~Hz}$. AFM images were further processed by Nova image processing software.

\subsection{Results and Discussion}

\section{Extinction and Absorption Cross Sections of Supported Silver Nanocubes:}

Optical cross sections of plasmonic nanoparticles are fundamental parameters for quantifying the strength of nanoparticle-light interactions and thus are important to measure experimentally. To do so, monolayers of silver nanocubes of two sizes (40 and 80nm average edge lengths) were deposited on various dielectric substrates under conditions preventing interparticle coupling (Figure 6.2). Average cube sizes and distributions for each sample were determined via TEM and confirmed by AFM and dipolar peak positions in the solution 
extinction spectra. The images in Figure 6.2 are typical of monolayers deposited on all substrates demonstrating that a single layer deposition of nanocubes was achieved. It is important to note that due to the nature of lateral AFM measurements distances between particles appear smaller than they actually are. UV-visible spectroscopy was employed to show that no coupling peaks were observed in the extinction spectra for all samples indicating interparticle interactions in a particular monolayer are negligible. As such only the particles and their interactions with the substrate are measured. Since the particle surface density is found from the AFM topography measurements (Figure 6.2), it is then possible to calculate the average extinction and absorption cross sections for a single nanocube. It is important to note that although the nanocubes are in a single layer with controlled interparticle distances they have a random rotational alignment perpendicular to the substrate. As such they are giving an average response that includes all rotational alignments. This consideration should also be extended to the substrate itself with respect to the average effective refractive index of the local environment below each of the cube. Figure 6.3 presents results of such calculations for two nanocube sizes on glass with 0,5 , and 20nm titanium oxide films thin films (the stoichiometry of the film is unknown, as such films are labelled as $\mathrm{TiO}_{\mathbf{x}}$ ). 
A: size $80 \mathrm{~nm}$, density $18.9 \pm 2.6 \mathrm{NC} / \mu \mathrm{m}^{2}$

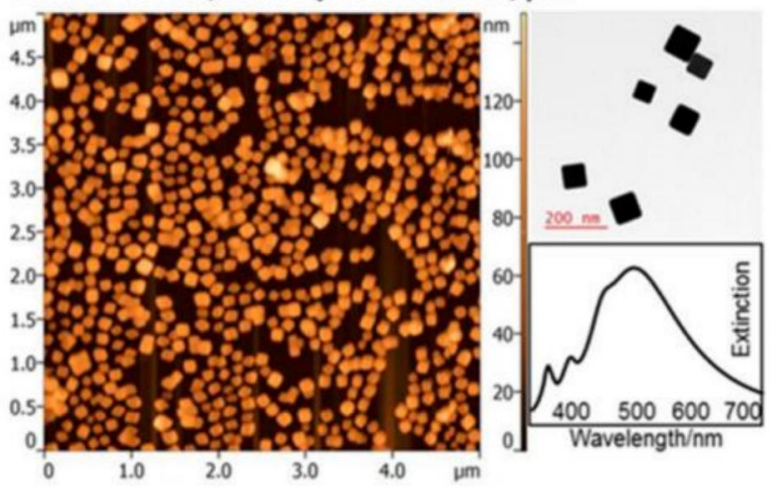

B: size $40 \mathrm{~nm}$, density $82.6 \pm 11.8 \mathrm{NC} / \mu \mathrm{m}^{2}$

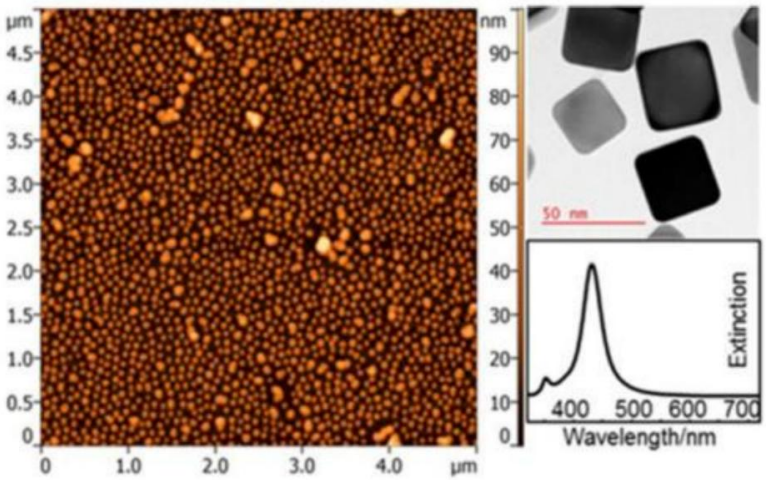

Figure 6.2. AFM topography and TEM images of 80nm (A) and 40nm (B) cubes deposited on a dielectric substrate demonstrate nanocube size and interparticle separation. Nanocube density is indicated. Extinction spectra of nanocubes in suspension are presented as well.

The cubic geometry of the nanoparticles provides a large nanoparticle-substrate contact area, leading to efficient hybridization of dipolar and quadrupolar plasmonic resonances, which appear in the spectra as two separate peaks, indicated D and Q, respectively (Figure 6.3). With increasing titanium oxide film thickness from 0 to $20 \mathrm{~nm}$, the effective refractive index of the substrate increases, shifting the dipolar resonance D to longer wavelengths while having little effect on the quadrupolar resonance Q.

Figure 6.3 presents extinction cross section spectra as solid lines, absorption for front illumination geometry as dotted lines, and absorption for rear illumination geometry as dashed lines. In addition, the physical cross sections calculated using the average nanocube sizes are shown as shaded areas. Two main conclusions can be made from Figure 6.3 data. First, the determined extinction and absorption cross sections are 2 to 5 times greater than their corresponding physical cross sections at the wavelength of maximum plasmonic resonance. The maximum extinction cross section for glass supported $80 \mathrm{~nm}$ cubes at $437 \mathrm{~nm}$ (corresponding to $\mathrm{D}$ resonance) was measured to be $\sim 19000 \mathrm{~nm}^{2}\left(\sim 0.019 \mu \mathrm{m}^{2}\right)$ which is almost 3 times greater than the average physical cross section of $6400 \mathrm{~nm}^{2}$. Similarly, for $40 \mathrm{~nm}$ cubes on glass the maximum extinction cross section measured at $415 \mathrm{~nm}$ is $3700 \mathrm{~nm}^{2}$ 
which is $\sim 2.5$ times greater than the corresponding physical cross section of $1600 \mathrm{~nm}^{2}$. The apparent increase in the cross sections for the 20nm films results from unaccounted light absorption by the substrate which becomes substantial for thicker films.

solid- extinction, dot- absorption front, dash- absorption rear, shaded- physical

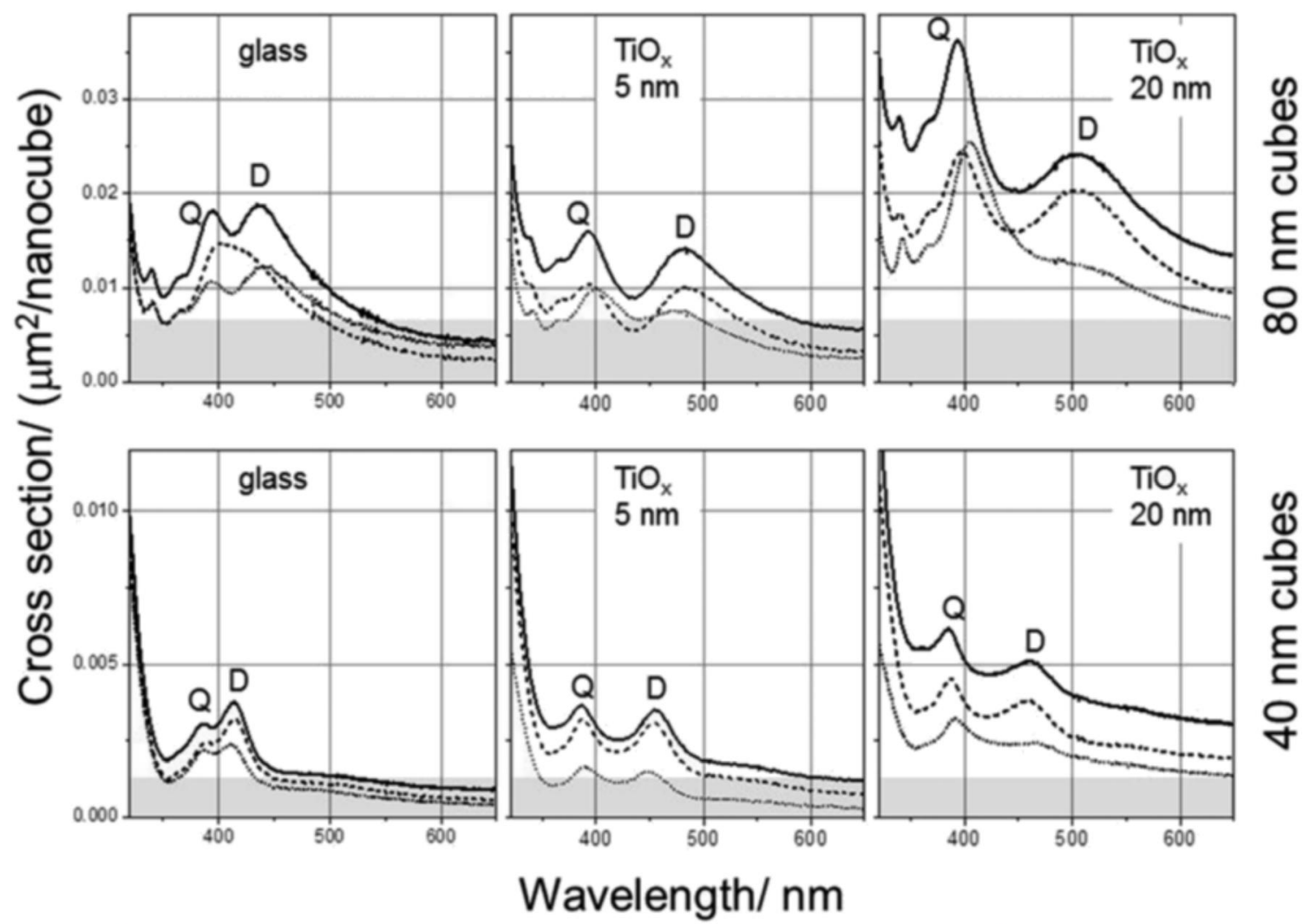

Figure 6.3. Cross sections of extinction (solid lines) and absorption in front (dotted lines) and rear (dashed lines) illumination geometry measured for $80 \mathrm{~nm}$ (top) and 40nm (bottom) silver nanocubes deposited on glass (left) and titanium oxide (5 and 20nm) substrates. Gray shaded areas indicate physical cross section. Quadrupolar and dipolar resonances are labelled.

These absolute cross sections are comparable with previously measured extinction and absorption cross sections for spherical silver nanoparticles. ${ }^{18}$ In that work the maximum extinction cross sections were found to be $5000-8000 \mathrm{~nm}^{2}$ for $30-40 \mathrm{~nm}$ and $30000-50$ $000 \mathrm{~nm}^{2}$ for $70-80 \mathrm{~nm}$ silver nanoparticles in suspension. The corresponding absorption cross sections are 4800 and $13000 \mathrm{~nm}^{2}$, respectively. The numbers are somewhat larger than in our 
present work. However, it should be noticed that our study investigated supported nanoparticles showing two resonances, instead of one. It is possible that the integral cross sections would be closer in values.

The second observation from the spectra is the dependence of the absorption cross section for cubes of both sizes on illumination geometry: front versus rear. Specifically, it appears that the hybrid dipolar resonance is anisotropic, whereas the quadrupolar resonance is not.

\section{Anisotropic Excitation of Dipolar Hybrid Resonance:}

The dipolar hybrid resonance appears to show anisotropic absorption cross sections depending on whether illumination occurs from the front or the rear of the substrate: it is considerably stronger in the rear illumination geometry (Figure 6.3). Interestingly, for both sizes of nanocubes, as the refractive index of the underlying substrate increases and the sample is illuminated from the front, the contribution of the hybrid dipolar mode to the absorption profile is increasingly diminished. This effect is the opposite when illuminated from the rear, yielding an ever increasing profile due to non-negligible titanium oxide film-light interactions with a strong similarity to the extinction spectra. Thus, it is apparent that the extent of the anisotropic behaviour of the dipolar resonance absorption depends on the refractive index of the supporting substrate.

A simple explanation of the anisotropic behaviour for the dipolar resonance can result from the fact that for front illumination geometry both reflected and transmitted light are absorbed by the dipolar and quadrupolar resonances. At the same time, for the rear illumination geometry the reflected light can be absorbed only by the dipolar resonance, since the quadrupolar resonance is located beyond the dielectric/air interface. As a result, the ratio of the dipolar to quadrupolar resonance intensities in the rear illumination geometry is 
enhanced compared to the front illumination geometry.

A more rigorous mechanism of the observed anisotropic dipolar absorption involves accounting for interaction between the incident (primary) light reflected at, or transmitted by, the substrate with one scattered by the nanocubes (secondary). As was shown previously, ${ }^{19}$ complex interference between the primary and secondary light could take place yielding enhanced and spatially very inhomogeneous electric field distribution at the interface. The interference pattern is strongly dependent on the wavelength of light and on illumination geometry due to the phase shift in the reflected light for rear illumination geometry. We hypothesize that for the rear illumination geometry the driving electric field experienced by the dipolar resonances is greater than for the front illumination geometry resulting in apparent enhanced absorption. Detailed calculations of the effect need to be performed to verify the hypothesis similar to those found in ref 19 . The study emphasizes a very strong distance dependence of the electric field from the interface that could result in different interaction of Q and D modes with light and also demonstrates a strong angular dependence very similar to the one observed in the present work.

This mechanism based on interference of primary and secondary waves was put forward to explain the anisotropic response of plasmonic solar cells, where constructive/destructive interference between the incident and scattered light in front illumination geometry was reported resulting in decreased photocurrent as compared to rear illumination geometry. ${ }^{10}$ This was due to enhanced local electric field at the plasmon resonance frequency and resulting generation of electron-hole pairs. As such the general mechanism is similar to the one we propose in the present study to explain the anisotropic absorption of light by dielectric supported nanocubes. 
for Supported Nanocubes: A very strong angle/polarization dependence of resonance excitation in supported nanocubes prompted us to investigate if absorption anisotropy is also sensitive to these parameters. ${ }^{15}$ Reflection and transmission data were measured for supported nanocubes on various dielectric supports as a function of angle for front and rear geometries with both s- and p-polarized light. Corresponding absorption spectra were further calculated (eq. 6.3). The data for the $5 \mathrm{~nm}$ titanium oxide film on glass substrate are presented in Figures 6.4-6.7. The spectra were measured for p- (Figures 6.4 and 6.6), and s(Figures 6.5 and 6.7) polarizations of incident radiation, in both rear and front geometries for 40nm (Figure 6.4 and 6.5) and 80nm (Figures 6.6 and 6.7) cubes at 16 different angles of incidence (0, 30, 35, 40, 45, 50, 52, 54, 56, 58, 60, 62, 64, 66, 68, and $70 \mathrm{deg})$.

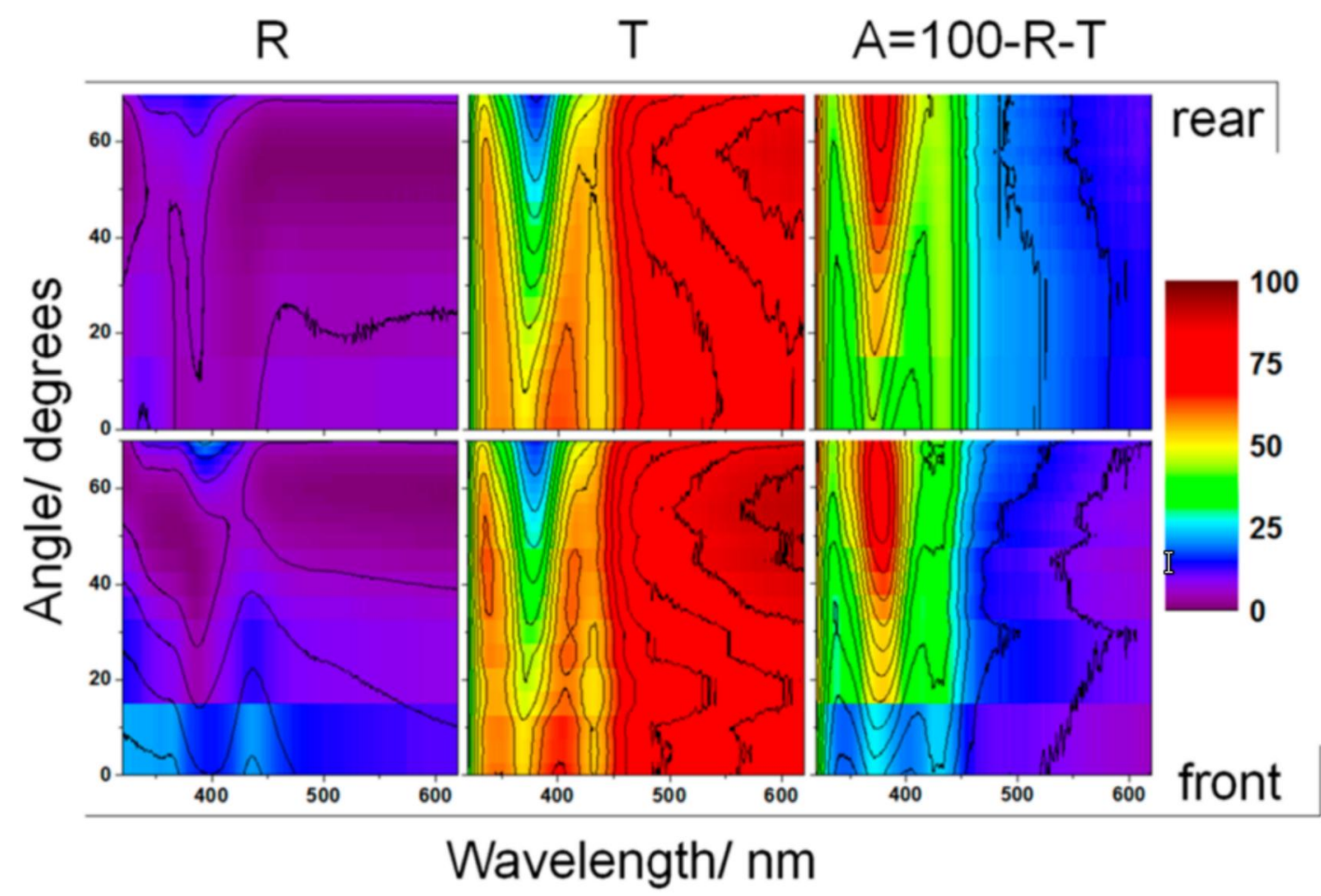

Figure 6.4. P-polarized light angle dependent reflection $(R)$, transmission $(T)$, and absorption $(A)$ for a $40 \mathrm{~nm}$ nanocube sample on a $5 \mathrm{~nm}$ titanium oxide film. The signal is measured in $\%$ for both rear (top) and front (bottom) illumination geometries. 


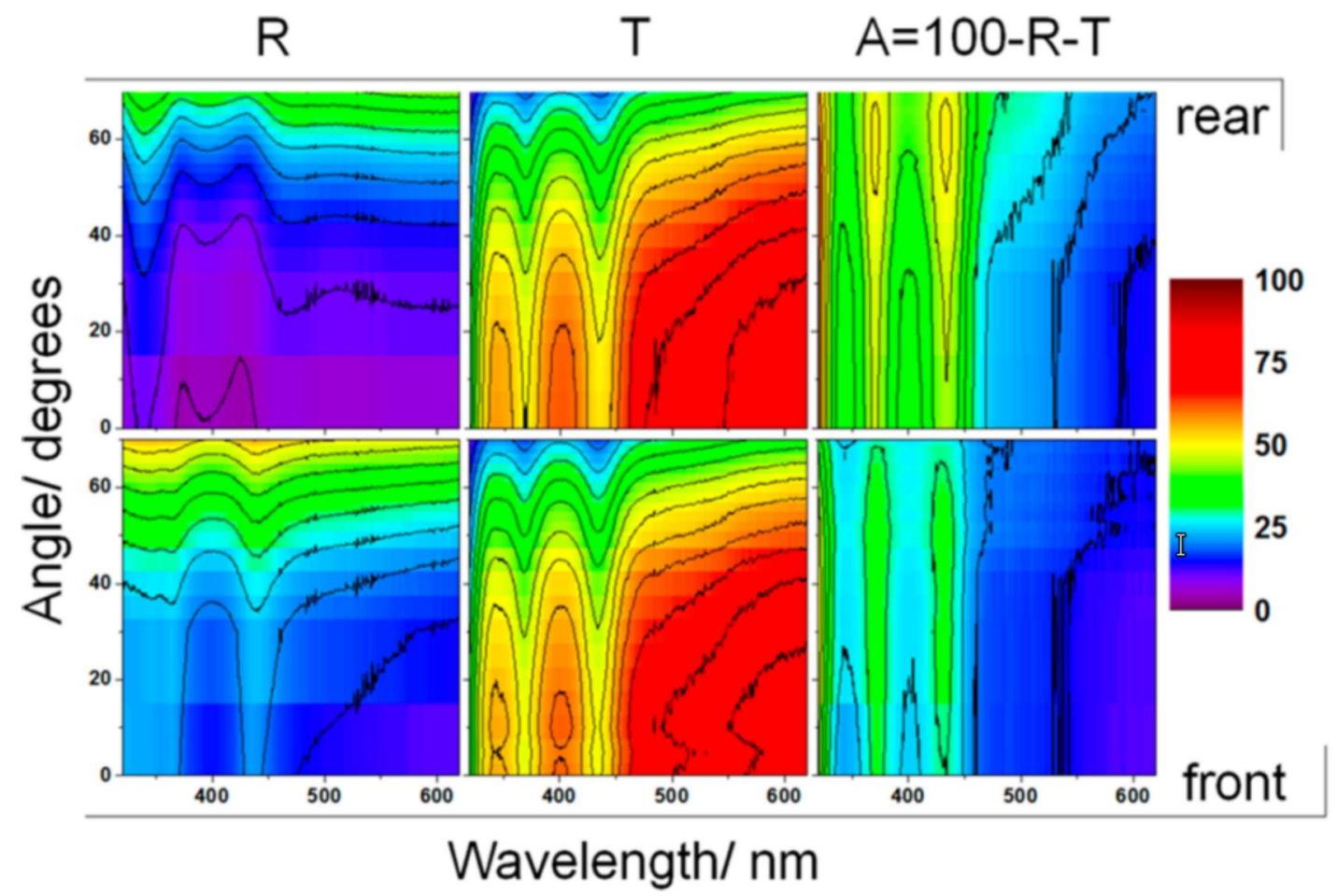

Figure 6.5. S-polarized light angle dependent reflection (R), transmission ( $T$ ), and absorption (A) for a $40 \mathrm{~nm}$ nanocube sample on a $5 \mathrm{~nm}$ titanium oxide film. The signal is measured in $\%$ for both rear (top) and front (bottom) illumination geometries.

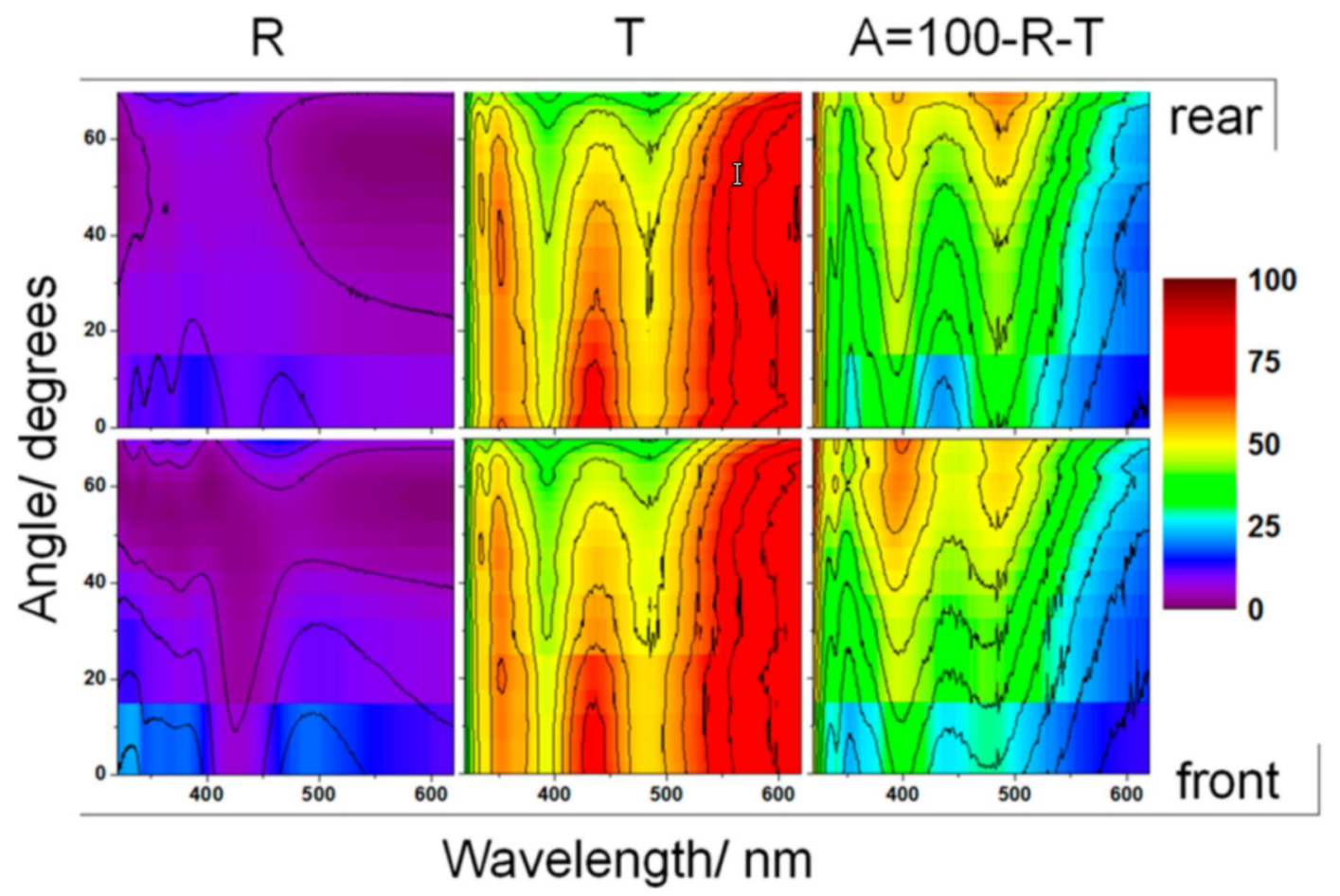

Figure 6.6. P-polarized light angle dependent reflection (R), transmission ( $T$ ), and absorption $(A)$ for an $80 \mathrm{~nm}$ nanocube sample on a $5 \mathrm{~nm}$ titanium oxide film. The signal is measured in $\%$ for both rear (top) and front (bottom) illumination geometries. 


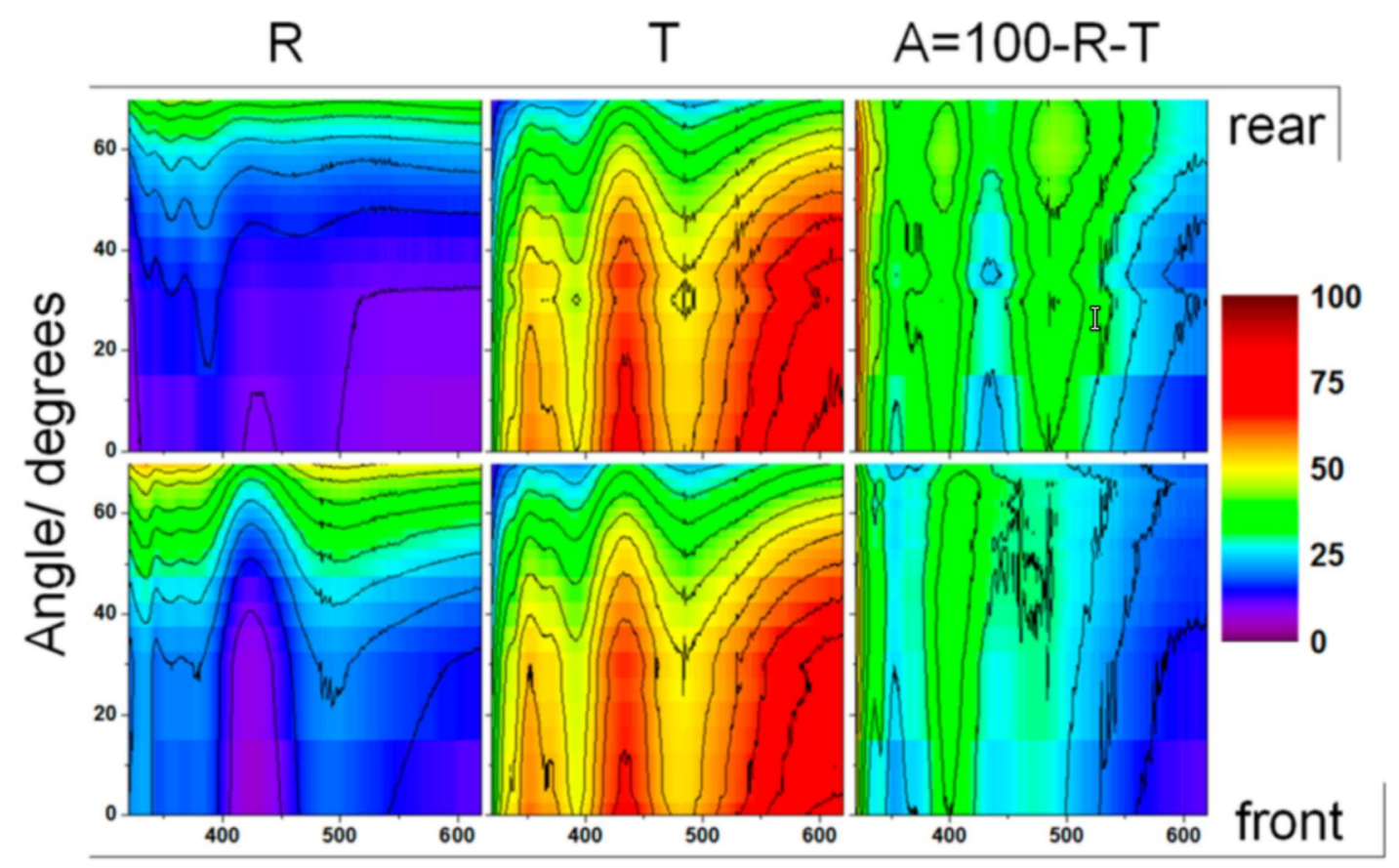

Wavelength/ $\mathrm{nm}$

Figure 6.7. S-polarized light angle dependent reflection (R), transmission ( $T$ ), and absorption $(A)$ for an $80 \mathrm{~nm}$ nanocube sample on a $5 \mathrm{~nm}$ titanium oxide film. The signal is measured in $\%$ for both rear (top) and front (bottom) illumination geometries.

Transmission of light (Figures 6.4-6.7, middle columns, T) is largely independent with respect to front and rear illumination yielding very similar maps for any particular cube size and polarization..$^{20,21}$ The major difference observed is for reflection measurements which vary significantly between front and rear illumination (Figures 6.4-6.7, left columns, R). Transmission and reflection spectra for both cube sizes show strong angle dependence, particularly when using p-polarized light. Consequently as the transmission spectra are largely invariable from front to back, making the dependencies observed in the reflection spectra the major contributors to anisotropy in the calculated absorption spectra using eq 6.3 (Figures 6.4-6.7, right columns, A).

The reflection spectra for both sizes of nanocubes show a strong dependency for ppolarized light as the angle of incidence is varied. The dependence comes from the 
plasmonic nature of the nanocubes but also from the angle dependent reflection of light from a dielectric surface. The minima in the reflection spectra are observed at $400 \mathrm{~nm}$ for $40 \mathrm{~nm}$ cubes (Figures 6.4, 6.5, columns R) and at about 430nm for 80nm nanocubes (Figures 6.6, 6.7, columns R) corresponding to their plasmonic resonances. On the other hand, the intensity of reflected p-polarized light is negligible at the Brewster angle (observed at 50-55 in Figures 6.4 and 6.6). Similarly, the monotonous increase of the intensity of spolarized light reflected at larger angles (Figures 6.5 and 6.7) is also well-known.

Transmission spectra contain the same contribution of the angle dependent reflection of light at the air-dielectric interface as the reflection spectra, in addition to the angle dependent reflection and absorption by the plasmonic resonances. In contrast the calculated absorption spectra (eq 6.3) only contain the absorption due to the plasmonic resonances, and thus the observed angle dependence of the absorption spectra is purely plasmonic in nature.

Several observations can be made from the absorption maps, shown in Figures 6.4-6.7 A: s- and p-polarized light have different sensitivities with respect to angle of incidence and a difference exists between small and large cubes, as well as between front and rear illumination geometries.

For s-polarized light, absorption does not seem to be sensitive to the angle of incidence for either nanocube size in addition to a stronger absorption for the rear illumination geometry (Figures 6.5, 6.7, right columns) especially at the dipolar resonance wavelength. The mechanism proposed above for normal spectra can explain the enhanced light absorption for the rear illumination geometry.

In contrast, for p-polarization the illumination geometry does not seem to play a major role, since the front and rear illuminated absorption maps are quite similar. However, the angle dependence is very significant, particularly for the smaller cubes (Figures 6.4, 6.6). 
It appears that with increasing angle of incidence absorption of light increases dramatically. For example, for smaller cubes the hybrid quadrupolar mode Q demonstrates a steady 3× increase in the intensity with increasing angle of incidence (Figure 6.4, right column) while the hybrid dipolar mode does not change. This is not the case for larger nanocubes, which show a constant increase (up to two times) in absorption for both modes with increasing angle of incidence (Figure 6.6, right column). This latter fact has not been reported before. It appears that for the smaller nanocubes there are fewer plasmonic states that could be excited by the p- polarized light, whereas these states are not as discrete for the larger cubes, and broadening of the resonances dominates the dependence.

An additional aspect to consider for such materials is how they behave as a waveguide. This is particularly relevant for use in photovoltaic enhancement where plasmonic particles are used to scatter light into the substrate and/or couple directly to the cell itself. While this work calculates absorption it does not differentiate between light that is scattered into the substrate or absorbed by the particles, making it difficult to determine if this particular configuration could yield a reasonable enhancement. However, an in depth understanding of the relationship between transmission, reflection, and absorption is required to minimize losses before considering the proportion of absorbed light that can be coupled into a waveguide. A recent study by Pennanen and Toppari revealed interference effects within the substrate itself as well as anisotropic response with respect to illumination geometry similar to those observed here. ${ }^{21}$

\subsection{Conclusions}

In this work, the optical and physical cross sections of dielectric supported silver nanocubes (40 and 80nm in edge length) were determined. The behaviour of these optical materials was also observed with respect to polarization, angle of incidence, and illumination 
geometry (front and back). A strong anisotropic behaviour for the hybrid dipolar mode for reflection and absorption with respect to illumination geometry was observed. The angle dependence of particular modes were also shown to vary with size, as the hybrid dipolar resonance of smaller cubes appears to be insensitive to increasing angle of incidence, while the opposite is true for the larger cubes. This work demonstrates that the transmission of light is largely insensitive to the above variables, showing that changes that occur in the reflection spectra dominate changes in the absorption spectra. The strong dependence of the plasmonic response of silver nanocubes on the supporting dielectric substrate can prove as a useful tool toward developing and understanding more efficient and effective plasmonic materials.

\subsection{References}

1 Noguez, C. Surface Plasmons on Metal Nanoparticles: The Influence of Shape and Physical Environment. J. Phys. Chem. C 2007, 111, 3806-3819.

2 Knight, M. W.; Wu, Y.; Lassiter, J. B.; Nordlander, P.; Halas, N. J. Substrates Matter: Influence of an Adjacent Dielectric on an Individual Plasmonic Nanoparticle. Nano Lett. 2009, 9, 2188-2192.

3 Ringe, E.; McMahon, J. M.; Sohn, K.; Cobley, C.; Xia, Y.; Huang, J.; Schatz, G. C.; Marks, L. D.; Van Duyne, R. P. Unraveling the Effects of Size, Composition, and Substrate on the Localized Surface Plasmon Resonance Frequencies of Gold and Silver Nanocubes: A Systematic Single-Particle Approach. J. Phys. Chem. C 2010, 114, 12511-12516.

4 Sherry, L. J.; Chang, S.-H.; Schatz, G. C.; Van Duyne, R. P. Localized Surface Plasmon Resonance Spectroscopy of Single Silver Nanocubes. Nano Lett. 2005, 5, 2034-2038.

$5 \quad$ McMahon, J. M.; Wang, Y.; Sherry, L. J.; Van Duyne, R. P.; Marks, L. D.; Gray, S. K.; Schatz, G. C. Correlating the Structure, Optical Spectra, and Electrodynamics of Single Silver Nanocubes. J. Phys. Chem. C 2009, 113, 2731-2735.

6 Lovera, A.; Gallinet, B.; Nordlander, P.; Martin, O. J. F. Mechanisms of Fano Resonances in Coupled Plasmonic Systems. ACS Nano 2013, 7, 4527-4536.

7 Zhang, S.; Bao, K.; Halas, N. J.; Xu, H.; Nordlander, P. Substrate- Induced Fano 
Resonances of a Plasmonic Nanocube: A Route to Increased-Sensitivity Localized Surface Plasmon Resonance Sensors Revealed. Nano Lett. 2011, 11, 1657-1663.

8 Jayawardhana, S.; Rosa, L.; Juodkazis, S.; Stoddart, P. R. Additional Enhancement of Electric Field in Surface-Enhanced Raman Scattering due to Fresnel Mechanism. Sci. Rep. 2013, 3, 2335.

$9 \quad$ Beck, F. J.; Polman, A.; Catchpole, K. R. Tunable Light Trapping for Solar Cells Using Localized Surface Plasmons. J. Appl. Phys. 2009, 105, 114310.

10 Beck, F. J.; Mokkapati, S.; Polman, A.; Catchpole, K. R. Asymmetry in Photocurrent Enhancement by Plasmonic Nanoparticle Arrays Located on the Front or on the Rear of Solar Cells. Appl. Phys. Lett. 2010, 96, 033113.

11 Powell, A. W.; Wincott, M. B.; Watt, A. A. R.; Assender, H. E.; Smith, J. M. Controlling the Optical Scattering of Plasmonic Nanoparticles Using a Thin Dielectric Layer. J. Appl. Phys. 2013, 113, 184311.

12 Spinelli, P.; Ferry, V. E.; van de Groep, J.; van Lare, M.; Verschuuren, M. A.; Schropp, R. E. I.; Atwater, H. A.; Polman, A. Plasmonic Light Trapping in Thin-Film Si Solar Cells. J. Opt. 2012, 14, 024002.

13 Moreau, A.; Ciracì, C.; Mock, J. J.; Hill, R. T.; Wang, Q.; Wiley, B. J.; Chilkoti, A.; Smith, D. R. Controlled-Reflectance Surfaces with Film-Coupled Colloidal Nanoantennas. Nature 2012, 492,86-89.

14 Lei, D. Y.; Fernández-Domínguez, A. I.; Sonnefraud, Y.; Appavoo, K.; Haglund, R. F.; Pendry, J. B.; Maier, S. A. Revealing Plasmonic Gap Modes in Particle-on-Film Systems Using Dark-Field Spectroscopy. ACS Nano 2012, 6, 1380-1386.

15 Bottomley, A.; Prezgot, D.; Staff, A.; Ianoul, A. Fine Tuning of Plasmonic Properties of Monolayers of Weakly Interacting Silver Nanocubes on Thin Silicon Films. Nanoscale 2012, 4, 6374-6382.

16 Ahamad, N.; Bottomley, A.; Ianoul, A. Optimizing Refractive Index Sensitivity of Supported Silver Nanocube Monolayers. J. Phys. Chem. C 2012, 116, 185-192.

17 Skrabalak, S. E.; Au, L.; Li, X.; Xia, Y. Facile Synthesis of Ag Nanocubes and Au Nanocages. Nat. Protoc. 2007, 2, 2182-2190.

18 Evanoff, D. D.; Chumanov, G. Size-Controlled Synthesis of Nanoparticles. 2. Measurement of Extinction, Scattering, and Absorption Cross Sections. J. Phys. Chem. B 2004, 108, 13957-13962.

19 Lermé, J.; Bonnet, C.; Broyer, M.; Cottancin, E.; Manchon, D.; Pellarin, M. Optical Properties of a Particle above a Dielectric Interface: Cross Sections, Benchmark Calculations, and Analysis of the Intrinsic Substrate Effects. J. Phys. Chem. C 2013, 
117, 6383-6398.

20 Mokkapati, S.; Beck, F. J.; de Waele, R.; Polman, a; Catchpole, K. R. Resonant NanoAntennas for Light Trapping in Plasmonic Solar Cells. J. Phys. D. Appl. Phys. 2011, 44, 185101.

21 Pennanen, A. M.; Toppari, J. J. Direct Optical Measurement of Light Coupling into Planar Waveguide by Plasmonic Nanoparticles. Opt. Express 2013, 21 (Suppl 1), A23-35. 


\section{Chapter 7}

Dynamics of nanocubes embedding into polymer films investigated via spatially resolved plasmon modes

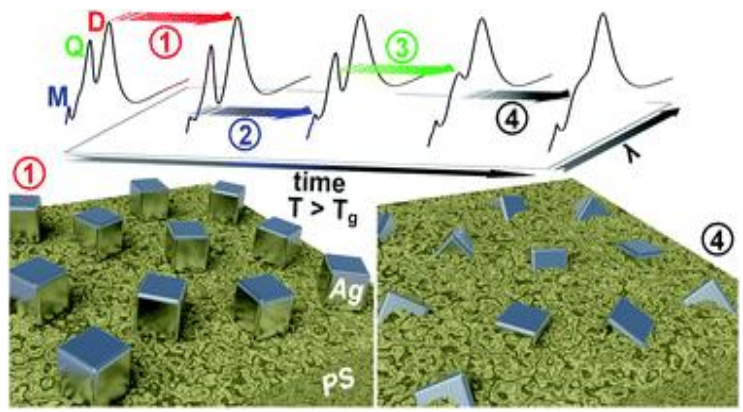


Modified from original manuscript published as:

Bottomley, A., Prezgot, D., Coyle, P. J., and Ianoul, A., Dynamics of nanocubes embedding into polymer films investigated via spatially resolved plasmon modes. Nanoscale 2016, 8 , 11168-11176.

Department of Chemistry, Carleton University, 1125 Colonel By Drive, Ottawa, Ontario, Canada, K1S 5B6

\subsection{Abstract}

Integration of nanoparticles into thin films is essential for the development of functional materials, studies of fundamental interfacial processes, and exploitation of inherent properties from the particles themselves. In this work, we systematically investigate the process of incorporation of silver nanocubes into thin polystyrene films at temperatures just above the polymer glass transition. The process of nanocrystal incorporation can be precisely monitored via far-field spectroscopy to observe the response of spatially resolved hybrid plasmon modes. Each plasmon resonance has a distinct dynamic range and maximum sensitivity forming a complementary set of nanorulers that operates over a distance comparable to the edge length of the cubes. The approach explored in this work is a general robust method for the development of long-range polychromatic nanorulers.

\subsection{Introduction}

The range of applications of noble metal nanoparticles is extensive due to their ability to support localized surface plasmons yielding intense optical properties and extremely high sensitivities to the material, geometry, and local environment. ${ }^{1}$ Their optical properties are tunable across the ultraviolet to the infrared, finding use in applications such as fluorescence enhancement, ${ }^{2}$ surface-enhanced Raman spectroscopy, ${ }^{3}$ catalysis, ${ }^{4,5}$ sensing, ${ }^{6}$ and photovoltaic enhancement. ${ }^{7}$ They also lend themselves as effective nanorulers ${ }^{8-11}$ for sub-diffraction measurements that can be performed using simple far-field observations 
while achieving spatial resolutions on the nanometer and, in some cases, angstrom scale. ${ }^{12-14}$ The high sensitivity to changes in the local environment surrounding plasmonic nanoparticles can be used to investigate phenomena such as the glass transition of various polymer films, allowing direct investigations into the thermodynamics of polymer interfaces. ${ }^{15-22}$ These types of investigations are of importance as polymers are a common choice for solid supported nanomaterials.

Precise control over the relative orientation and distances of nanocrystals is critical in nanoscale engineering and design when the geometry of individual components in various nanoarchitectures defines their useful properties. In this respect, achieving the ability to continuously monitor nanoscale assembly processes over large distances with high precision remains a desirable yet challenging goal. To address these needs a wide variety of nanorulers utilizing various interactions and properties of plasmonic nanomaterials have been explored. This includes rulers based on coupling interactions between adjacent particles, ${ }^{8,9,13,23,24}$ Fano interferences as a result of the interaction, ${ }^{8,25}$ and particle coupling with metal surfaces. ${ }^{12,14,26}$ Another common approach to plasmonic-based nanorulers utilizes the relationship between a plasmon and an emitter of some kind, including distance dependent surface enhanced Raman spectroscopy,${ }^{27}$ nanometal surface energy transfer interactions, ${ }^{10,28,29}$ and plasmophore surface enhanced fluorescence. ${ }^{30}$ The resolution, range, sensitivity, and ease of fabrication of nanorulers varies considerably, in some cases yielding angstrom resolution for interparticle/substrate coupling bands, while other systems can attain up to $100 \mathrm{~nm}$ in dynamic range such as the plasmophore based systems. A common element of the nanorulers described above is the interaction of a plasmonic particle with a specific nearby object; often this is another metal particle or surface or an emitter such as a fluorophore or a Raman label. Consequently, many of these systems need to be specifically calibrated for every 
circumstance as they are extremely sensitive to small changes in shape, orientation of the components, and the local environment of the system itself. Alternatively some nanorulers are simply based on the change in the refractive index of their surrounding environment. Refractive index sensing with inherent plasmonic modes does not need a coupling band or interaction with an emitter to function. These simple nanorulers have been shown to accurately determine the thickness of dielectric spacers up to $30 \mathrm{~nm} .^{31}$

Of particular interest, due to their ability to support strong hybridized modes ${ }^{32}$ silver nanocubes are an excellent candidate for use as a nanoruler and an optical reporter in composite materials, as individual nanocubes are highly sensitive to anisotropy in their local environment. ${ }^{33}$ Thus, it is possible to gain significant control over the optical properties of silver nanocubes while also gaining insight into the position, orientation, and degree of anisotropy in their local refractive index at any time. The spatially separated modes that occur when silver nanocubes are placed in an anisotropic environment allow for the resulting hybrid modes to be tuned independently for a small range in the visible spectrum. This level of control and information feedback about the conditions surrounding the nanocrystals makes this platform an effective tool for the production of composite materials with specific properties.

In the present work, we investigate how the sensitivities of hybrid plasmonic modes generated by anisotropy in the refractive index of the surrounding environment can yield significant control over the spectral signature of ensembles of silver nanocubes. In addition to spectral control the response of these hybrid modes to their changing environment allows them to be used as a set of three complementary nanorulers giving a combined dynamic range of $\sim 60 \mathrm{~nm}$. This is accomplished without the use of interparticle or substrate coupling bands or the use of emitters such as fluorophores or Raman labels. 


\subsection{Experimental}

\section{Chemicals}

Silver nitrate $\left(\mathrm{AgNO}_{3}, 99 \%+\right)$, polyvinylpyrrolidone (PVP, $\mathrm{M}_{\mathrm{w}} \sim 55$ 000), polystyrene (PS, $\mathrm{M}_{\mathrm{w}} \sim 192$ 000), anhydrous 99.8\% ethylene glycol (EG), sodium sulfide, toluene, and chloroform were purchased from Sigma-Aldrich and used as obtained. Ethanol (95\%) was obtained from Commercial Alcohols and used without further purification. 1,2Dioleoyl-sn-glycero-3-phosphocholine (DOPC) was purchased from Avanti Polar Lipids as a powder and used as obtained.

\section{Preparation of nanocubes}

Silver nanocubes were synthesized according to the procedure described in the literature. ${ }^{34}$ To obtain larger cubes, $25 \mu \mathrm{L}$ of $0.48 \mathrm{M}$ sodium chloride in EG was added to the reaction flask prior to the injection of silver nitrate. When the characteristic peak found at 350 nm was developed an additional $1.5 \mathrm{~mL}$ of $282 \mathrm{mM}$ silver nitrate was added at a rate of $0.75 \mathrm{~mL} \mathrm{~min}^{-1}$. The reaction was thermally quenched when the desired spectrum was observed.

\section{Preparation of polystyrene thin films}

Polystyrene was dissolved in toluene to produce $1 \%$ and $0.5 \% \mathrm{w} / \mathrm{w}$ solutions, which were then filtered through a $0.220 \mu \mathrm{m}$ PTFE filter. Thin films were produced via static spincoating on a Laurell WS-400-6NPP/LITE spin-coater. Films were allowed to anneal at 130 ${ }^{\circ} \mathrm{C}$ overnight.

\section{Preparation of AgNC monolayers}

Nanocube substrates were prepared by forming Langmuir films on a NIMA 311D trough filled with Milli-Q water (18.2 M $\Omega$ cm). An appropriate amount of AgNC and DOPC was dispersed in chloroform and deposited onto the surface of the water using a micro- 
syringe. Each monolayer was left for 20 min to allow complete chloroform evaporation. The surface pressure of the monolayer was measured with a paper Wilhelmy plate. Before transferring the monolayer onto solid supports several isotherm cycles were performed to anneal the films. Monolayers were transferred onto clean PS coated substrates at pressures corresponding to the gas phase of the isotherm via the Langmuir-Schaefer deposition method.

\section{Heating of supported monolayers of PS films}

A small steel block connected to a thermocouple was placed on top of a hotplate and used to heat substrates placed on it for specific intervals. Substrates were periodically imaged via AFM and UV-visible spectroscopy.

\section{UV-visible measurements}

Extinction spectra were obtained using a Cary 7000 equipped with a universal measurement analyzer. Spectra were processed and analyzed using OriginLab data analysis software. Multiple regions of the substrate were measured at each interval. Irregularities in particle distribution and/or the substrate can influence the peak widths and positions observed in the extinction spectrum, so the most occurring spectrum or statistical mode was considered as the actual measurement.

\section{Topographical measurements}

The topography of the NC monolayers transferred onto solid substrates was obtained using an Ntegra (NT-MDT, Russia) atomic force microscope in semi-contact mode in air at $21{ }^{\circ} \mathrm{C}$ with $512 \times 512$ or $1024 \times 1024$ points per image. A $100 \times 100 \mu \mathrm{m}^{2}$ scanner (Ntegra) and cantilevers with rotated monolithic silicon tips (135 $\mu \mathrm{m}$ long, $0.3-6 \mathrm{~N} \mathrm{~m}^{-1}$ spring constant, NSG03, resonance frequency $90 \mathrm{kHz}$, NT-MDT) were used for all topographic measurements. The typical scan rate was $0.5 \mathrm{~Hz}$. AFM images were further processed by 
using Nova image processing software.

\section{Numerical modelling}

Finite difference time domain modelling was performed using Lumerical Solutions Inc. (FDTD Solutions v8.15). The simulation objects consisted of a $62 \mathrm{~nm}$ silver nanocube with a corner radius of $12.5 \mathrm{~nm}$ and a $2 \mathrm{~nm}$ capping layer of PVP supported by a $150 \mathrm{~nm}$ film of PS supported by an arbitrarily large $\mathrm{SiO}_{2}$ substrate extended to the boundaries of the simulation (800 nm). The materials Ag, 35,36 PVP, 37 and PS 38 were described by an analytical model fitted to tabulated permittivity data. A mesh override region extending at least $10 \mathrm{~nm}$ past the nanocube was used with a $0.5 \mathrm{~nm}$ mesh size. A total-field scattered-field (TFSF) light source was used, and a perfect matched layer (PML) was used for the boundary conditions.

\subsection{Results and Discussion}

\section{Spectral effects of embedding nanocubes into polystyrene}

Supported silver nanocubes show very characteristic extinction spectra that depend on the size of the cubes and the dielectric properties of the support. ${ }^{39,40}$ There are two hybrid peaks present in the spectra assigned as dipolar and quadrupolar surface plasmon resonances. These hybrid modes are at their sharpest when the cube is not coupling to another metal surface. Plasmonic coupling produces a peak at a longer wavelength while also making the inherent peaks more broad and less distinguished. ${ }^{41,42}$

In this work nanocubes with the average edge length of $62 \pm 11 \mathrm{~nm}$ (Fig. 7.1) were studied. The size distribution is not particularly narrow; however the size dependence of the position of the quadrupolar resonance is relatively weak $^{43}$ and the corresponding UV-vis extinction spectrum shows characteristic sharp signatures with relatively narrow half widths when on a substrate. The dipole (D) resonance for the nanocubes while in solution is at 481 
$\mathrm{nm}$ with the measured full width at half maximum (FWHM) of around $90 \mathrm{~nm}$ (Fig. 7.1A).

Other higher order resonances present in the solution spectrum are at 393 and $346 \mathrm{~nm}$. The E-field distribution corresponding to these modes is shown as insets in Fig. 7.1A and appears to be symmetrical.

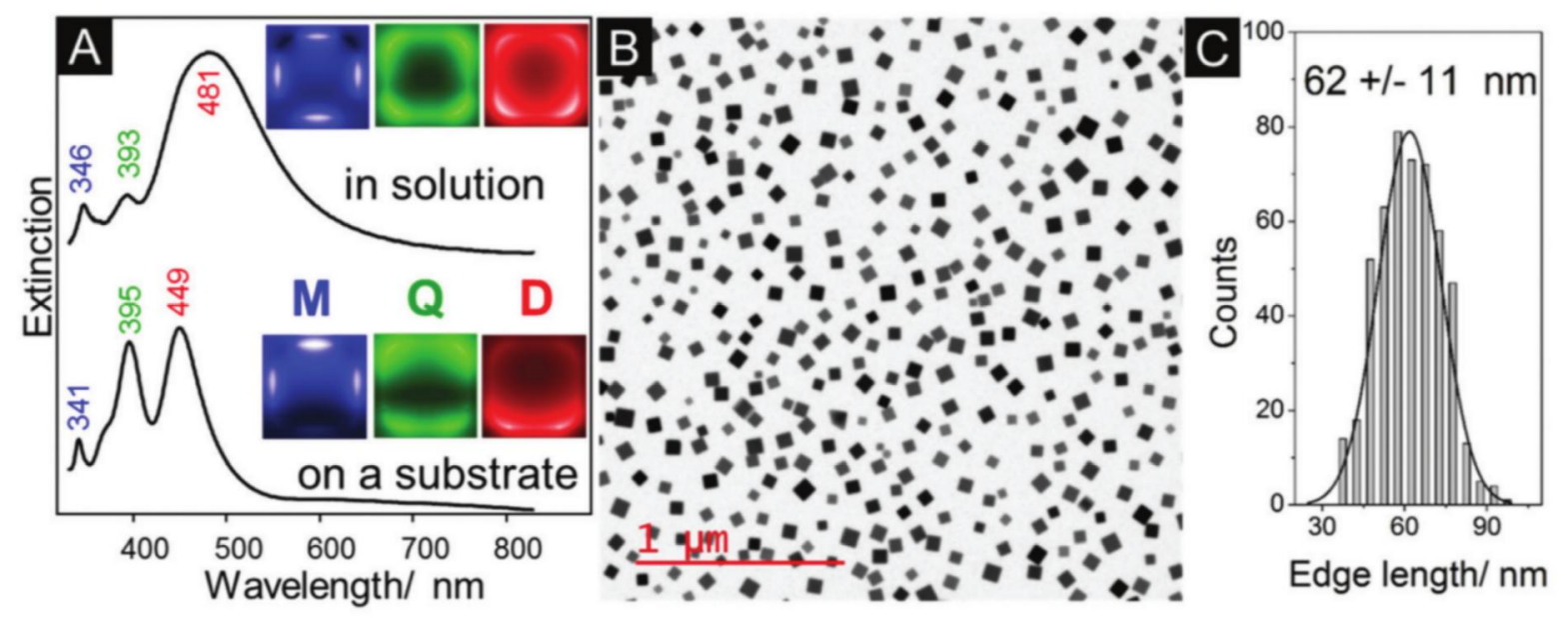

Figure 7.1. (A) UV-visible extinction spectra of a nanocube sample in ethanol (top) and on a polystyrene substrate in air (bottom). E-field distributions for the dipolar (D), quadrupolar (Q), and multipolar (M) resonances are shown for both environments. (B) TEM image of as synthesized cubes. (C) Histogram showing size distribution in terms of edge length for the sample.

When supported by a thin polystyrene (PS) film the cubes exhibit a narrow hybrid dipolar resonance $^{32}(\mathrm{D})$ at $449 \mathrm{~nm}(\mathrm{FWHM} \sim 40 \mathrm{~nm})$, a hybrid quadrupolar resonance (Q) at $395 \mathrm{~nm}$ (FWHM $30 \mathrm{~nm}$ ), and a multipolar resonance (M) at $341 \mathrm{~nm}(14 \mathrm{~nm})$ (Fig. 7.1A). Assignment and spatial distribution of the resonances have been studied theoretically and experimentally, for example using electron energy loss spectroscopy ${ }^{44-47}$ and cathodoluminescence. ${ }^{48}$ The E-field distribution maps (Fig. 7.1A, inset) show that the E-field associated with the hybrid dipolar resonance is primarily located at the bottom part of the cube, quadrupolar at the top, and multipolar resonances have their significant component associated with the sides of the cube. This spatially resolved nature of plasmonic modes as well as small FWHMs enables us to precisely monitor the dynamics and changing local environment around the nanocubes as they embed into their supporting polymer. 
At room temperature PS exists in the glassy state and can therefore act as a dielectric substrate smooth enough to support nanocube monolayers. Bulk PS experiences a glass transition at $\sim 100{ }^{\circ} \mathrm{C} .{ }^{49}$ While the transition temperature depends on factors such as the chain length and tacticity, a significant depression in the $\mathrm{T}_{\mathrm{g}}$ to $\sim 75{ }^{\circ} \mathrm{C}$ occurs for ultra-thin films. ${ }^{50}$ Extinction spectra of the nanocube monolayers are allowed to evolve over time when the system is kept above the supporting PS glass transition temperature at $112 \pm 1{ }^{\circ} \mathrm{C}$ (Fig. 7.2). The observed changes do not result from nanocube transformation, such as rounding or oxidation, ${ }^{43}$ but from their response to being embedded into the PS film. Exclusion of thermally induced damage was addressed by comparing the PS supported sample to a glass supported sample. The glass supported cubes, heated alongside the PS supported cubes, showed no changes in their extinction spectrum at the end of the experiment. Therefore, the observed spectroscopic evolution is associated with the nanocrystals embedding into the polystyrene film exclusively.
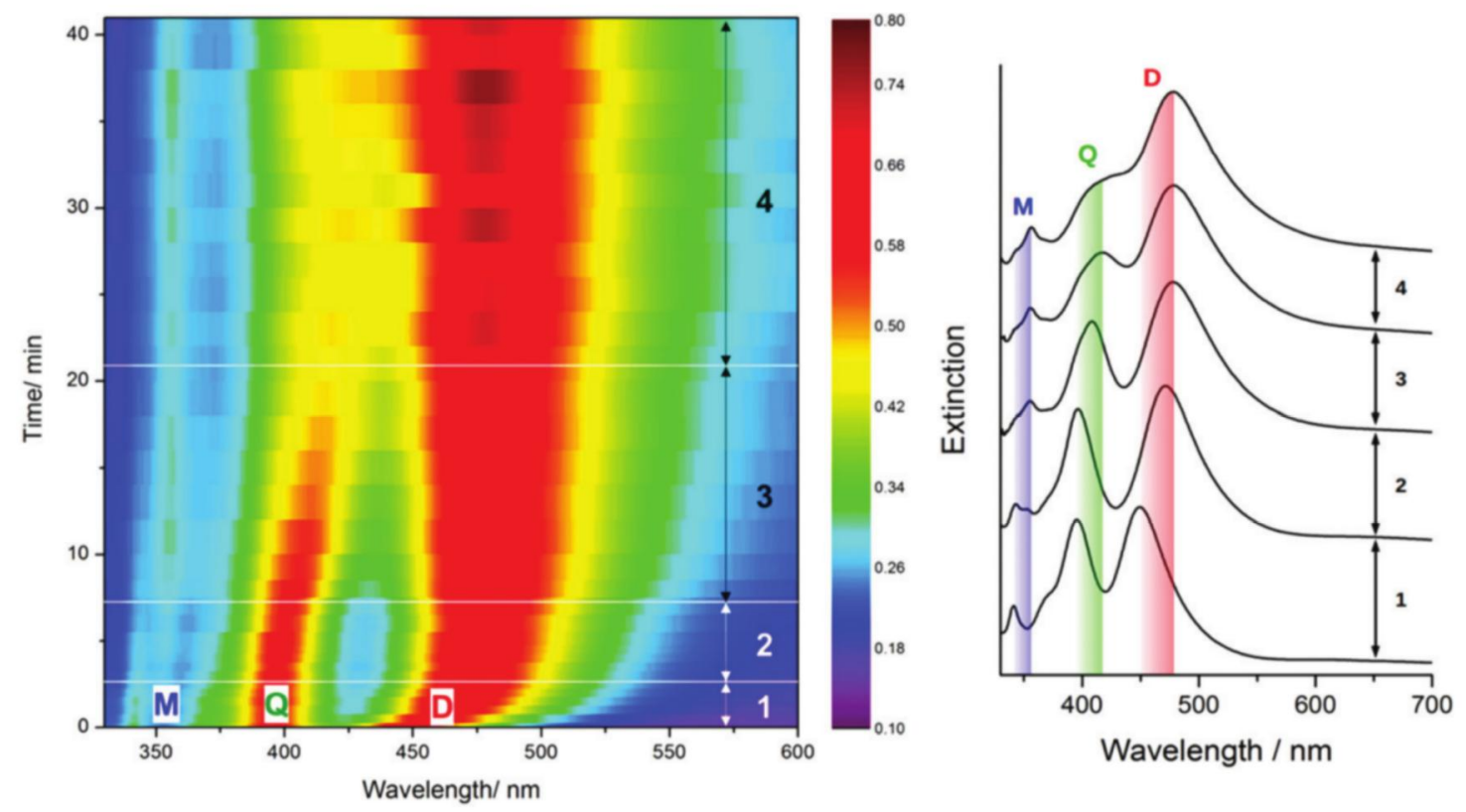

Figure 7.2. (Left) Contour plot depicting the attenuation of light as a function of time. Contributions from plasmon modes are labelled as dipolar (D), quadrupolar (Q), and multipolar (M). (Right) Single extinction spectra corresponding to the horizontal lines on the contour plot. The red, green, and blue bands indicate the overall shift for $\mathrm{D}, \mathrm{Q}$, and $\mathrm{M}$ modes respectively. 
The spectroscopic changes that occur with time can be separated into the four stages shown in Fig. 7.2. The first stage is characterized by a very strong red-shift of the D resonance from the original $449 \mathrm{~nm}$ to $480 \mathrm{~nm}$. Both the position and intensity of the Q resonance remain almost invariant. In the $\mathrm{M}$ resonance region, a peak around $350 \mathrm{~nm}$ is developed in addition to the original peak at $341 \mathrm{~nm}$.

During the second stage both D and Q resonances slightly red-shift. At the same time, the $\mathrm{M}$ resonance shows a two state-like transition between the peaks at $\sim 341 \mathrm{~nm}$ and 350 nm. During the third stage, the D resonance reaches a max. at $485 \mathrm{~nm}$, Q shows the greatest red-shift to $\sim 414 \mathrm{~nm}$, and the resonance at 340 disappears, leaving only a $350 \mathrm{~nm}$ multipolar resonance in the region. Finally, during the fourth stage the D and $\mathrm{M}$ resonances remain unchanged. At the same time, the Q resonance becomes obscured by the overlap with the D mode.

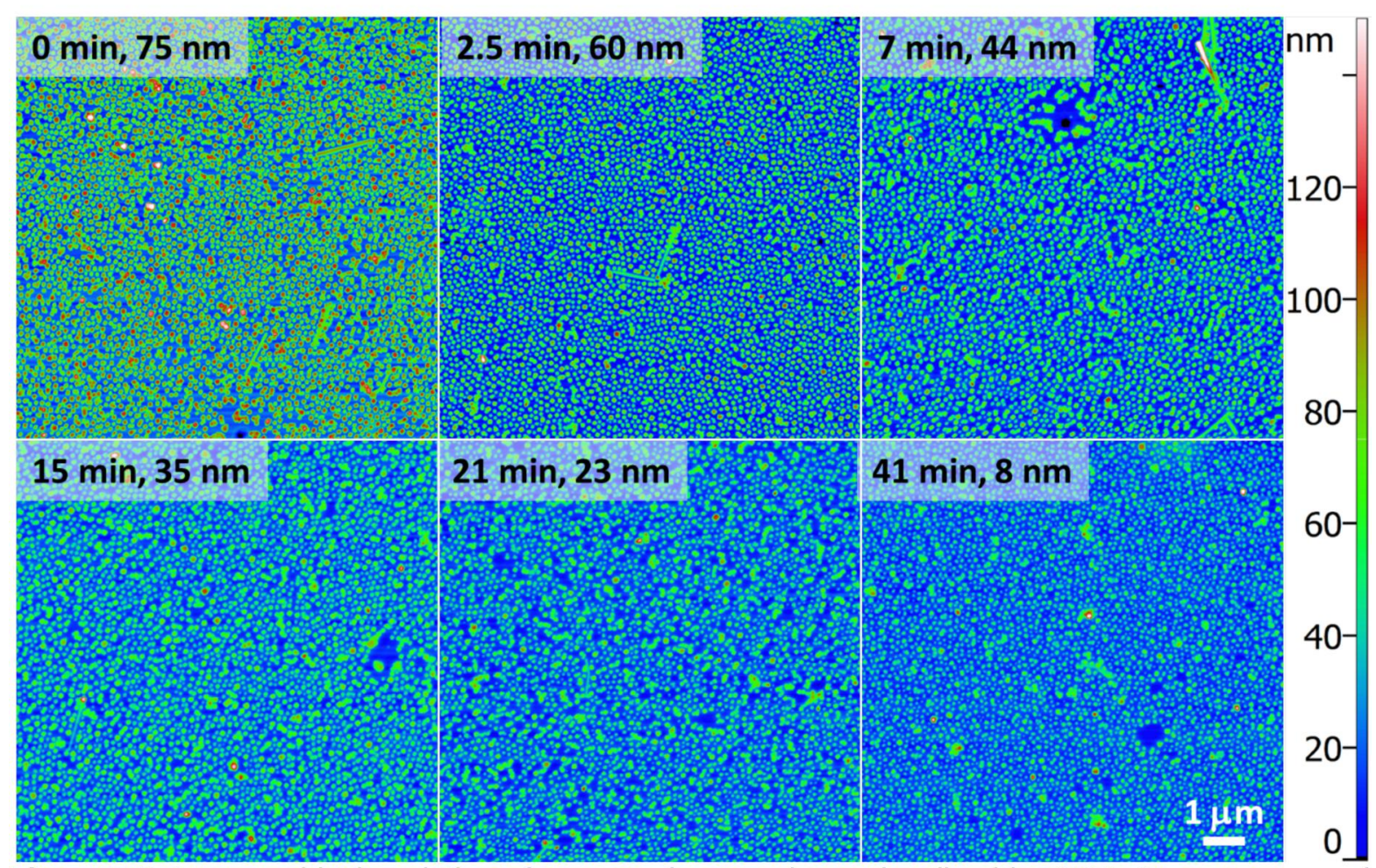

Figure 7.3. Topographical evolution of a nanocube monolayer visualized by AFM. Average exposed cube height and duration of heating are indicated for each panel. 
The spectral changes can be reasonably well explained by the process of incorporation of nanocubes into the PS film upon heating as observed by AFM (Fig. 7.3 and 7.4). As a result of incorporation, the environment surrounding the nanocubes becomes more isotropic and the spectrum moves towards that of nanocubes in a high refractive index solution as hybridization of the plasmonic modes is reduced.
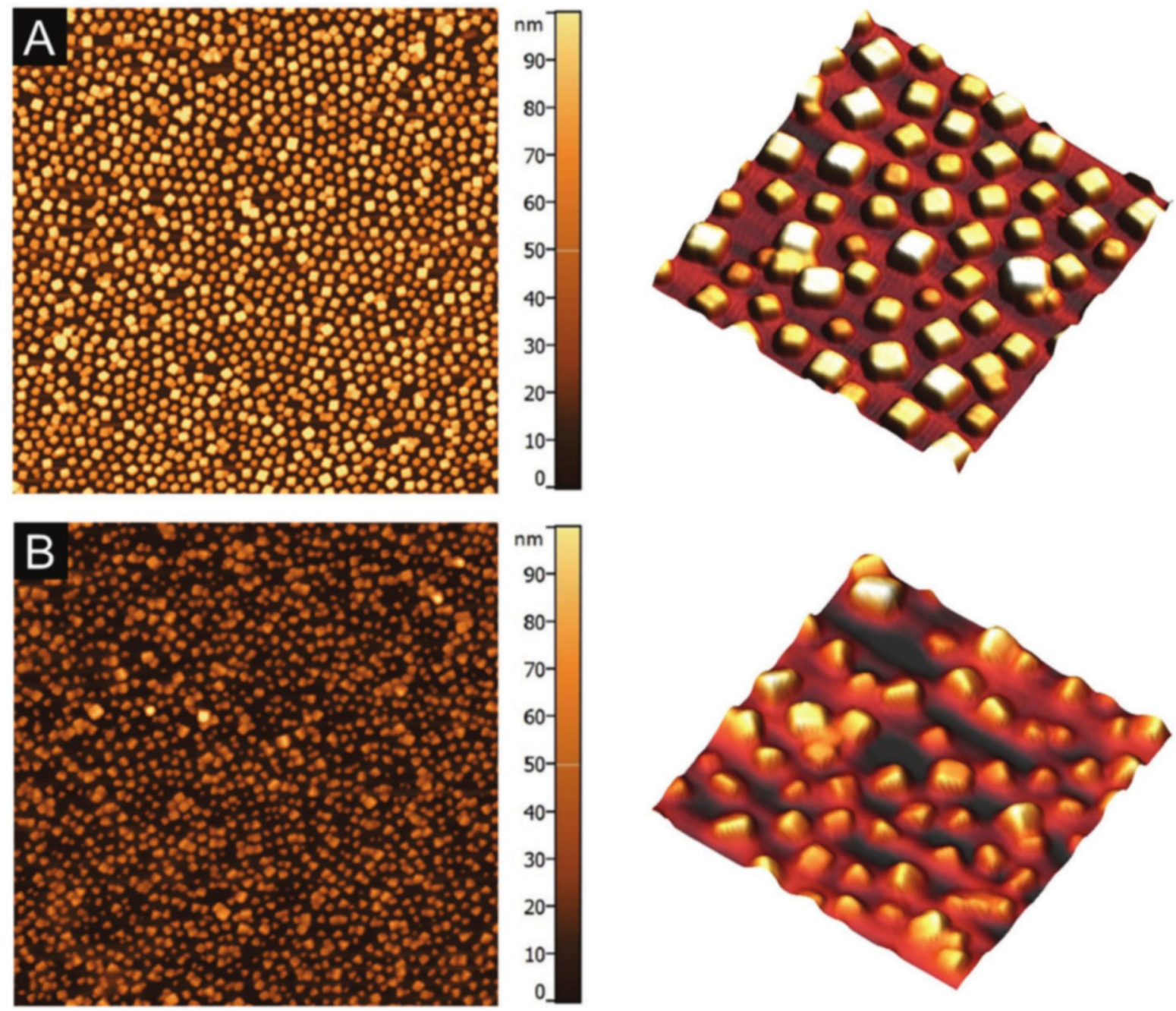

Figure 7.4. AFM images ( $5 \times 5 \mu \mathrm{m}^{2}$, left, and $1 \times 1 \mu \mathrm{m}^{2}$, right) of nanocube monolayers (A) initially and $(B)$ after heating for $30 \mathrm{~min}$ at $112{ }^{\circ} \mathrm{C}$.

\section{Extinction spectrum as a nanoruler}

Detailed AFM measurements of the nanocube monolayer for each stage of 
spectroscopic changes help us to better determine the relationship between the geometry of the system and its optical signature. According to AFM measurements, the average height of the nanocube monolayer decreases with heating time (Fig. 7.3). The original untreated monolayer showed an average height of $75 \mathrm{~nm}$ above the substrate (Fig. 7.4A and 7.3). This is somewhat greater than the average size of the nanocubes as determined from TEM (Fig. 1, $62 \mathrm{~nm}$ ). The discrepancy can be accounted for by the PVP layer around the nanocube, which can be $1-4 \mathrm{~nm}$ in thickness, ${ }^{51}$ and imperfections of the topographic analysis. Upon further heating for $15 \mathrm{~min}$, the average height of the monolayer decreases to $\sim 50 \%$ of the nanocube edge length. After 40 min of heating, the average height of the cubes within the monolayer is only around $8 \mathrm{~nm}$.

It is possible to correlate the spectral changes in the NC/PS system to the average exposed height of the nanocrystals. For example, in this particular experiment (under specific heating conditions and geometry) the four stages described above and exemplified by Fig. 7.2, 7.3 and 7.4 correspond to the following changes in the AFM data:

1. During the first stage (less than 2 minutes of heating) the average height of the nanocubes within the monolayer decreases from $75 \mathrm{~nm}$ to $55 \mathrm{~nm}$ while retaining their orientation and spatial arrangement. This region has the highest overall sensitivity and is dominated by the peak shift of the dipolar resonance.

2. During the second stage the average height of the exposed nanocubes decreases to $\sim 45 \mathrm{~nm}$, occurring between 2 and 7 minutes of heating. The most sensitive observable for this stage is the emergence of a pair of multipolar (M) modes at $\sim 341$ and $350 \mathrm{~nm}$. As the particles embed further into the substrate, the full transition of peak intensity from the higher energy to the lower energy $M$ mode occurs. Additionally a portion of the cube population begins to experience a tilting motion during this stage. 
3. The third stage (7-21 minutes) sees further incorporation of the cubes into the polymer layer. The average height decreases below $30 \mathrm{~nm}$, which is roughly half the nanocube size. By this point nearly all of the cubes have experienced some type of tilting motion. During this stage, the sensitivity of the Q mode is at its greatest.

4. The final stage in this experiment corresponds to almost complete submergence of the particles. The average height decreases to less than $25 \mathrm{~nm}$ with the majority of cubes having only a single corner exposed. This stage has the least sensitivity of the four and is associated with a loss of anisotropy in the local environment around the cubes.

This analysis clearly indicates that with the help of three independent and spatially separated spectroscopic markers $\mathrm{D}, \mathrm{Q}$, and $\mathrm{M}$ it is possible to monitor the position of embedded nanoparticles over the distance of $\sim 60 \mathrm{~nm}$. The maximum sensitivity for each of the markers occurs at 0,22 , and $36 \mathrm{~nm}$ depth for the $\mathrm{D}, \mathrm{M}$, and $\mathrm{Q}$ modes respectively and therefore provides an opportunity to measure not only very short distances, but also short lateral displacements over an extended range greater than any of the modes individually.

For a nanocube on top of the PS film (embedding depth $0 \mathrm{~nm}$ ) the E-field is distributed around the bottom, middle and top of the supported cube observed for the D, M, and Q resonances respectively (Fig. 7.1A). As the nanocube embeds into the polymer the three resonances sense changes in the environment during different time intervals (Fig. 7.5). The dependencies can be fairly well fitted with the mono-exponential decaying function shown in eqn (1):

$$
\text { Relative Signal Increase }=I_{0}-I_{1} e^{-\left(t-t_{0}\right) / \tau}
$$

where $I_{0}, I_{1}$, and $t_{0}$ are fitting parameters to give characteristic time constants $\tau$ of 1.6, 9.3, and 23.4 minutes for $\mathrm{D}, \mathrm{M}$, and $\mathrm{Q}$ responses respectively. 


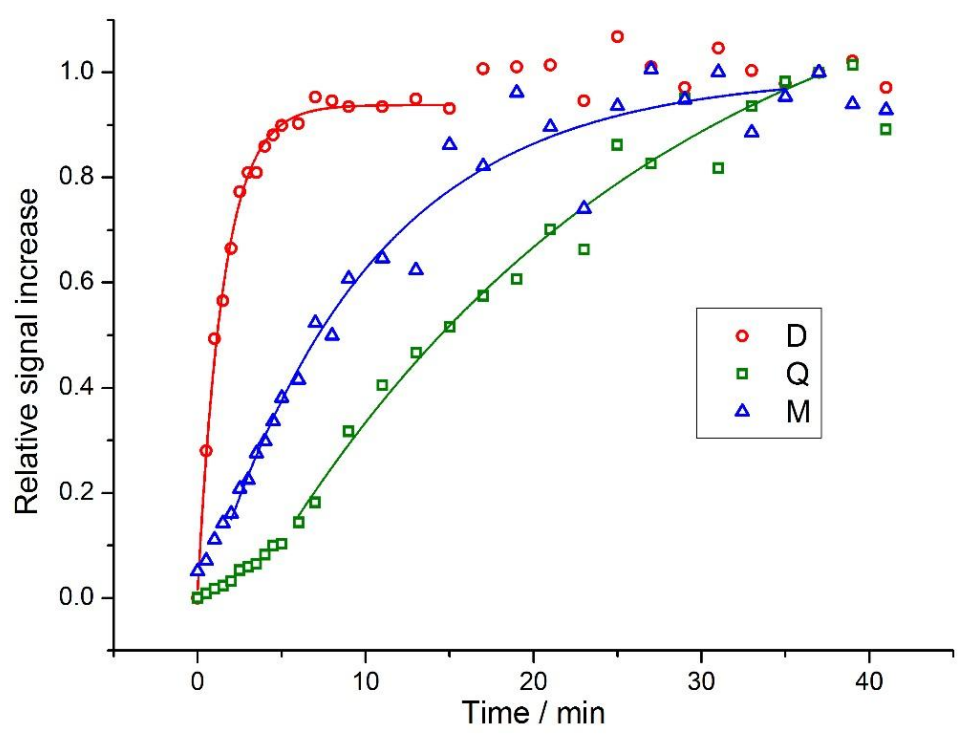

Figure 7.5. Evolution of plasmonic modes. Relative change in wavelength of the dipolar resonance (red circles, relative change in intensities of the multimode resonances (blue triangles), and relative change in the wavelength of quadrupolar resonance (green square) versus time.

As the rate of incorporation of the nanocrystals into the polymer film is a function of temperature and the glass transition temperature of a thin polymer film is dependent on many factors such as film thickness, ${ }^{50}$ we plot the relative spectral features as a function of depth of immersion of the cubes into the film to yield a relationship that is dependent only on the position of the cubes. Fig. 7.6 shows the normalized relative response of peak positions for the $\mathrm{D}$ and $\mathrm{Q}$ modes, and the relative intensity of the $\mathrm{M}$ modes. Dipolar and quadrupolar relationships can be expressed as peak shift in nanometers over distance in nanometers, yielding sensitivities in units of $\mathrm{nm} \mathrm{nm}^{-1}$ (spectral/physical). The dipolar resonance shows the greatest sensitivity at the initial point of the experiment of $2.1 \mathrm{~nm}$ $\mathrm{nm}^{-1}$, decreasing exponentially as the cubes become further imbedded into the polymer. Initially, the Q mode is insensitive to the changing environment around the cube, but rapidly increases in sensitivity after $\sim 20 \mathrm{~nm}$ of immersion to a maximum of $1.2 \mathrm{~nm} \mathrm{~nm}-1$ at $36 \mathrm{~nm}$ depth, roughly half the edge length of the cubes. The transition between the $\mathrm{M}$ resonances is not one of spectral shift, but the disappearance of one mode and the emergence of the other 
and thus sensitivity is the percentage change in $\mathrm{nm}^{-1}$ and is strongly dependent on the sensitivity of the instrumentation used for measurements. This transition has a dynamic range between 10 and $35 \mathrm{~nm}$ of embedding with a maximum sensitivity found at $22 \mathrm{~nm}$. This maximum corresponds to the condition when both multipolar resonances are present and of equal intensity and conveniently supplements the low point in observed sensitivity at the intersection of the D and Q modes.

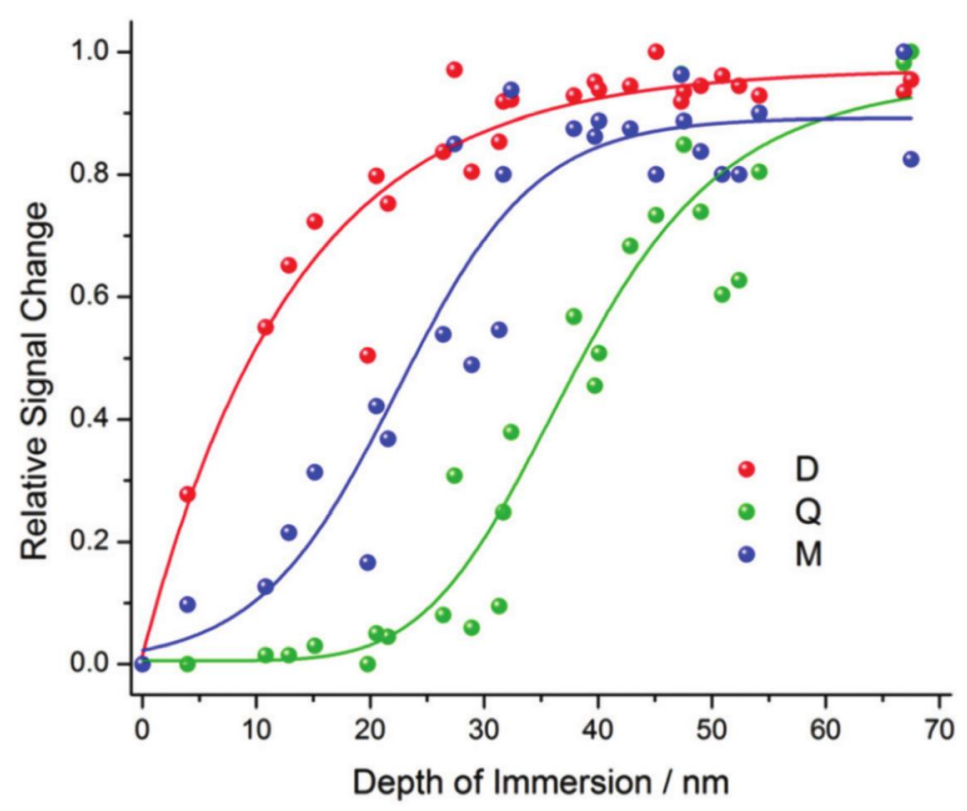

Figure 7.6. Evolution of plasmonic modes as a function of nanocrystal depth. Red and green data points correspond to the normalized peak shift versus particle depth for the dipolar (D) and quadrupolar (Q) modes respectively. The blue data points represent the relative change in intensities of the multimode $(\mathrm{M})$ resonances versus depth of nanocube immersion. Fitted lines are empirical and based on a mono-exponential decay for $\mathrm{D}$, and logistic functions for $\mathrm{Q}$ and $\mathrm{M}$ modes.

\section{Free energy change on embedding a particle}

The driving force for incorporation of nanocubes into a PS film is described by the Helmholtz free energy decrease $A_{s}$ as determined by Kovacs et al. ${ }^{15,16,22}$ If we assume that the initial and final states are $\mathrm{A}_{\mathrm{s}}=a \gamma_{1}$ and $\mathrm{A}_{\mathrm{s}}=a \gamma_{12}$ where $a$ is the area, and $\gamma_{1}$ and $\gamma_{12}$ are the surface energies of a non-interacting nanocrystal surface and the interface between the 
nanocrystal and the substrate, the change in surface free energy is described by eqn (2) such that if $\gamma_{1}>\gamma_{12}$, then $\Delta A_{s}<0$ and the interaction is spontaneous:

$$
\Delta \mathrm{A}_{\mathrm{s}}=\mathrm{a}\left(\gamma_{12}-\gamma_{1}\right)
$$

Satisfying eqn (2) is sufficient to determine whether particles will begin to incorporate themselves into the polymer, but not sufficient to predict whether particles will become fully embedded. If the system reaches a local minimum at any point before the particles become fully embedded, the sinking process will cease. ${ }^{15}$ If we consider the reformation of the polystyrene surface with energy $\gamma_{2}$ as the particle continues to sink, it becomes apparent that to remain spontaneous the surface energy of the particle must be greater than the sum of the interfacial surface energy and the polymer surface energy, yielding inequality (3):

$$
\gamma_{1}>\gamma_{2}+\gamma_{12}
$$

During this experiment, we did not observe the full immersion of nanocubes into polystyrene even at extended times in excess of 1 hour and elevated temperatures up to 140 ${ }^{\circ} \mathrm{C}$. As such, this system fails to satisfy inequality (3) and suggests that we observed the intermediate regime that is described by inequality (4):

$$
\gamma_{12}<\gamma_{1}<\gamma_{2}+\gamma_{12}
$$

This equation states that the surface tension of the underlying polymer is simply too high relative to the particle surface energy to allow the particles to fully embed themselves. This conclusion is consistent with the experimental observations of other groups who have succeeded in fully embedding silver nanocubes into polystyrene only by solvent annealing under vacuum, ${ }^{52}$ which effectively lowers the surface tension of the polymer, satisfying inequality (3). Additionally, observing this regime is a direct consequence of utilizing 
surface passivated nanocrystals. The surface energies of bulk polyvinylpyrrolidone ${ }^{53}$ and polystyrene $^{54}$ are similar at $\sim 43$ and $33 \mathrm{~mJ} \mathrm{~m}^{-2}$ respectively and significantly lower than that of bare metals. For example, Kovacs et al. ${ }^{15}$ lists the surface energy for bare silver at 1100 $\mathrm{mJ} \mathrm{m}^{-2}$, which is two orders of magnitude higher than the surface energy of polystyrene, effectively forcing inequality (3) to be always true for an unpassivated silver particle. The mechanism for fully embedding particles is often reported with the generation of a wetting layer around $1 \mathrm{~nm}$ thick. ${ }^{17,18}$ This does not occur in our experiment as this would satisfy inequality (3) and lead to fully embedded particles. This relationship sheds some light on the surface energy of the interface between the particle and the polymer, and also opens experimental avenues to produce nanocomposites that allow for control over physical access to the surface of the nanocrystals.

\section{Spectra of partially embedded cubes: modelling}

To investigate the process in more detail, the finite-domain time-difference (FDTD) method was used to model the extinction spectra of a nanocube embedded iteratively in 5 nm steps into a polystyrene film (Fig. 7.7). The nanocube’s orientation as well as the light source's angle of incidence were kept normal to the substrate. As the nanocube is lowered into the substrate a rapid shift in the D mode is initially observed while the $\mathrm{Q}$ remains relatively insensitive; the $\mathrm{D}$ mode shifts $\sim 60 \mathrm{~nm}$ while the $\mathrm{Q}$ mode only shifts $\sim 5 \mathrm{~nm}$ at 30 $\mathrm{nm}$ of immersion. As the cube is further immersed in the substrate the sensitivity of the D mode gradually diminishes as the Q mode's sensitivity increases until it reaches a maximum at $55 \mathrm{~nm}$ of immersion. This results in a red-shift of the Q mode by $\sim 50 \mathrm{~nm}$ between $30 \mathrm{~nm}$ and $60 \mathrm{~nm}$ of immersion, while the D mode only shifts $15 \mathrm{~nm}$ (Fig. 7.7). 


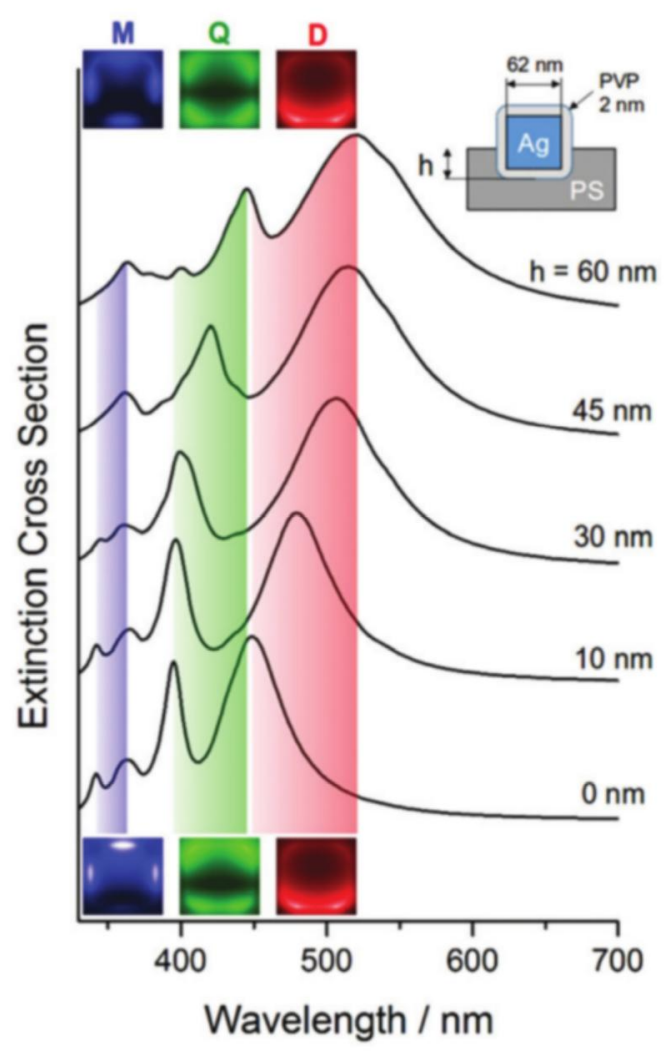

Figure 7.7. FDTD calculated extinction spectra of a silver nanocube in the polystyrene film for different embedding depths. E-Field distributions (imbedded) are given for the initial and final states of the simulation. The red, green, and blue bands indicate the overall shift for $\mathrm{D}, \mathrm{Q}$, and $\mathrm{M}$ modes respectively.

In general this model reliably follows the trends observed experimentally. When comparing sensitivities derived from the simulations to the experimentally observed values we find that the $\mathrm{D}$ mode is shown to have a maximum sensitivity of $4.0 \mathrm{~nm} \mathrm{~nm}^{-1}$ as opposed to $2.1 \mathrm{~nm} \mathrm{~nm}^{-1}$ while the $\mathrm{Q}$ reaches a maximum of $1.8 \mathrm{~nm} \mathrm{~nm} \mathrm{~nm}^{-1}$ as opposed to $1.2 \mathrm{~nm} \mathrm{~nm}^{-1}$. The increased sensitivity is a consequence of the model describing an idealized scenario involving only a single nanocube. The experimental measurements were performed on an ensemble of nanocubes which introduce a degree of polydispersity in terms of size, depth of immersion, and orientation, all of which contribute to band-broadening and consequently a reduction in the sensitivity when compared to the single particle model.

While general agreement between the model and experimental results was obtained, 
it should be noted that the model begins to diverge from the experimental results after the cube is immersed at a depth greater than half its edge length. While almost no further peak shift of the D mode was observed after the cubes were half immersed experimentally (Fig. 7.6), the model predicts a further peak shift of $20 \mathrm{~nm}$ during the second half of the experiment (Fig. 7.8A). The Q mode also shifts faster experimentally than predicted by the model; the predicted point of maximum sensitivity of the Q mode is after the nanocubes have been immersed $55 \mathrm{~nm}$, while we observe this point at $36 \mathrm{~nm}$ of immersion in the experiment. A shift to lower energy for the M mode is predicted by the model occurring after the cube has been half-immersed, while the actual transition observed experimentally occurs at a much earlier stage. Error tends to be significant in modelling the imaginary component of the dielectric function in this region of the spectrum for silver. The discrepancy is attributed to interference from the interband transition edge for silver $\sim 3.8 \mathrm{eV}(326 \mathrm{~nm})$ affecting a small spectral range near this position. ${ }^{36}$ For this reason we do not discuss the modelled results of the $\mathrm{M}$ mode in detail. The divergence between the model and experimental results primarily corresponds with our third stage of experimental observations where tilting of the nanocubes becomes significant. The model described thus far has consisted of a nanocube orientated normal to the substrate. It therefore becomes necessary to examine the effect of nanocube orientation on the optical properties of the particle in order to consolidate the predicted and experimental results. 

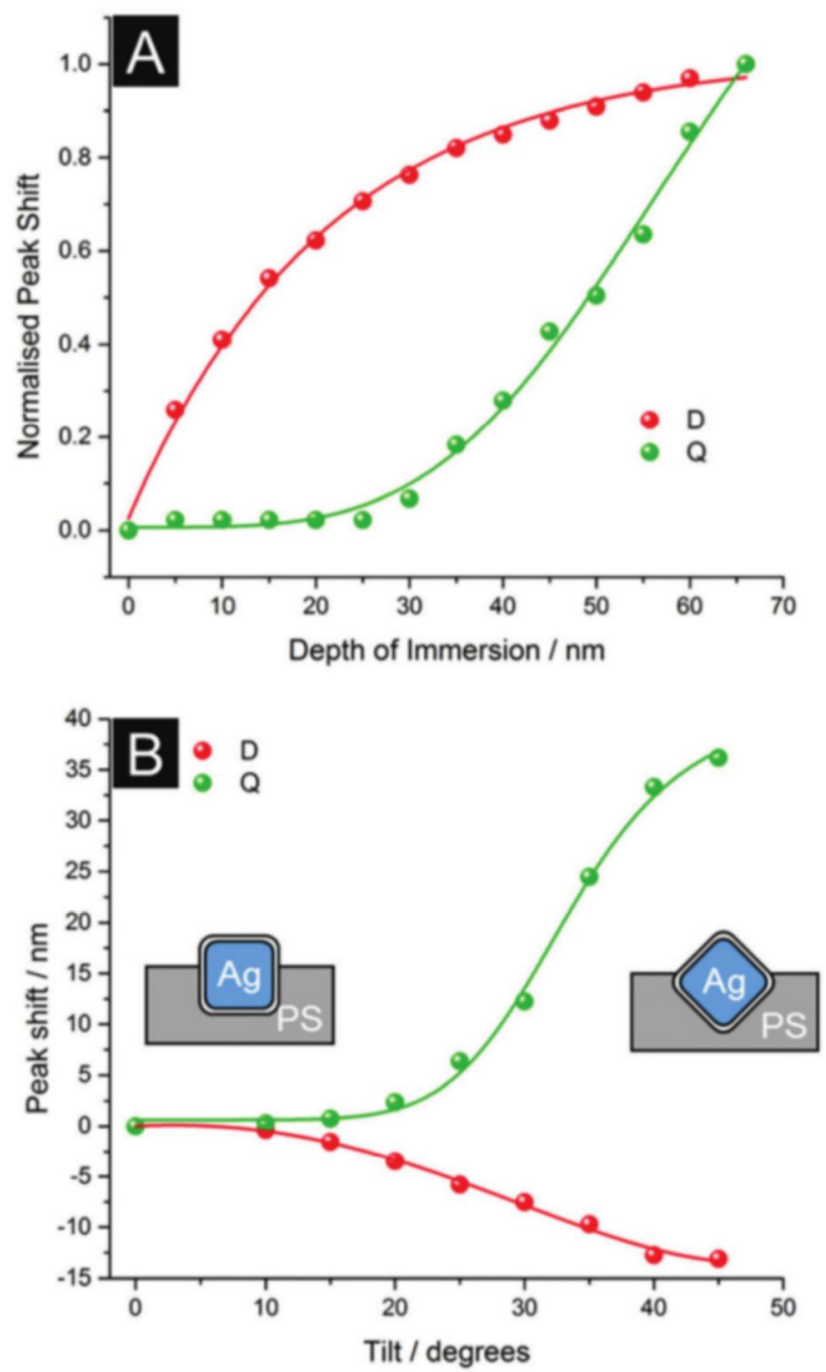

Figure 7.8. Peak shifts from FDTD modelling displaying: (A) normalized peakshift versus depth of immersion for a normal oriented $62 \mathrm{~nm}$ cube imbedding into polystyrene. (B) Relative peak shift against angle of tilt about a single axis for a $62 \mathrm{~nm}$ cube held at a constant depth of $40 \mathrm{~nm}$ in polystyrene. Very little change is observed at small angles $\left(<15^{\circ}\right)$, but increasing the angle of tilt results in a small blue shift of the $\mathrm{D}$ mode and a rapid red shift of the $\mathrm{Q}$ mode.

\section{Effects of nanocube orientation on extinction spectra}

FDTD simulations were utilized to independently examine the effect of nanocube orientation on their extinction spectra using an unpolarized light source orientated perpendicular to the substrate, which we define as the $\mathrm{z}$ axis. A series of simulations was performed on a cube immersed $40 \mathrm{~nm}$ into poly tyrene, which was rotated between $0^{\circ}$ and $45^{\circ}$ about a single axis through its center. Experimentally the cubes are free to rotate along 
three degrees of freedom in terms of the $\mathrm{x}, \mathrm{y}$ and $\mathrm{z}$ axes. Individual rotations about the $\mathrm{x}$ and $\mathrm{y}$ axes are degenerate in terms of spectral response while rotation about the $\mathrm{z}$ axis is unobservable as all orientations are present at the initial and final states of the experiment. We observe cubes having rotated about both the $\mathrm{x}$ and $\mathrm{y}$ axes at a variety of angles after 30 minutes of heating (Fig. 7.4), with the majority having rotated to large angles $\left(>25^{\circ}\right)$ on at least one of the two axes. Fig. 7.8B describes the peak shift of the D and Q modes as the cube is rotated about a single axis. A rotation of less than $15^{\circ}$ results in very little spectral response, but further rotation results in a blue shift of the D mode by $13 \mathrm{~nm}$ and a red shift of the Q mode up to $36 \mathrm{~nm}$ at $45^{\circ}$. By contrasting these spectral shifts against those associated with the normal-oriented cube model we illustrate that the experimental shifts in the final stages of the experiment arise from the combination of the nanocube immersion and the change in orientation which occur simultaneously. While a small ( $\sim 15 \mathrm{~nm})$ red shift of the D mode is predicted for the last $20 \mathrm{~nm}$ of immersion of a normal-oriented nanocube (Fig. 7.8A), the change in orientation of the nanocube results in a comparable blue shift. The competition between these phenomena leads to the observation of the apparent saturation of the shift of the D mode. Meanwhile, the red shift of the Q mode associated with the change in orientation of the nanocube accumulates with the red shift associated with further immersion of the nanocube. The combined effect leads to a more rapid shift of the Q mode than would be observed if the nanocube did not change orientation.

\section{Subfacial sensitivity of hybridized modes}

As long as there is refractive index contrast present in a system, spatially resolved plasmon modes can be used to track the changes in their surroundings. The initial optical signature and spectral evolution of the system as particles embed themselves into the polymer layer are dependent on the refractive index of the surrounding environment above 
the system, the polymer itself, and in cases where the particle can migrate near the bottom of the polymer film the refractive index of the underlying solid support will have a significant impact on the spectral features of the film. As the system evolves, it moves from a strongly hybridized regime resulting from high anisotropy to a more isotropic system that results in less hybridization as the cubes embed themselves into the polymer layer. However, if the polymer layer is chosen such that it is thin enough so that cubes can nearly reach the bottom of it, and the film is situated on a substrate of relatively high refractive index it becomes possible to push the system back in the direction of hybridization by inducing anisotropy within the material. This scenario was experimentally explored by using a thin polystyrene film ( $\sim 50 \mathrm{~nm})$ on top of a high refractive index substrate of titanium oxide (Fig. 7.9). As a result of the increased refractive index of the substrate, the $\mathrm{D}$ mode does not saturate before the cubes stop embedding. As the relative refractive indices of both the substrate and the superstrate are higher than the initial setup at time zero, all modes are red shifted but maintain a degree of hybridization. This system adds an extra dimension to the nanoruler, extending its dynamic range. Similar improvements can be made by choosing a higher refractive index polymer, again increasing the sensitivity and dynamic range of the system. High-n polymers such as polyphosphazenes and polyphosphonates provide an attractive alternative to polystyrene due to their high refractive index, up to 1.75 compared to polystyrene's $n$ of 1.60 at $550 \mathrm{~nm}$ as well as a similar $T_{\mathrm{g}}$ and processability. ${ }^{55}$ FDTD modelling was employed to compare the refractive index sensitivity of the nanoruler on a polymer with $n=1.75$ against the polystyrene model. An improvement in the maximum sensitivity of both the D and Q modes was observed. The D mode's initial sensitivity increases from 4.0 to $5.0 \mathrm{~nm} \mathrm{~nm}^{-1}$, while the Q mode's maximum sensitivity increases from 1.8 to $2.2 \mathrm{~nm} \mathrm{~nm}^{-1}$ at $50 \mathrm{~nm}$ of immersion. With alternative material choices of both the 
substrate and the polymer film, provided the process of incorporation remains energetically favourable, it becomes possible to further improve the sensitivity and dynamic range for this type of nanoruler.

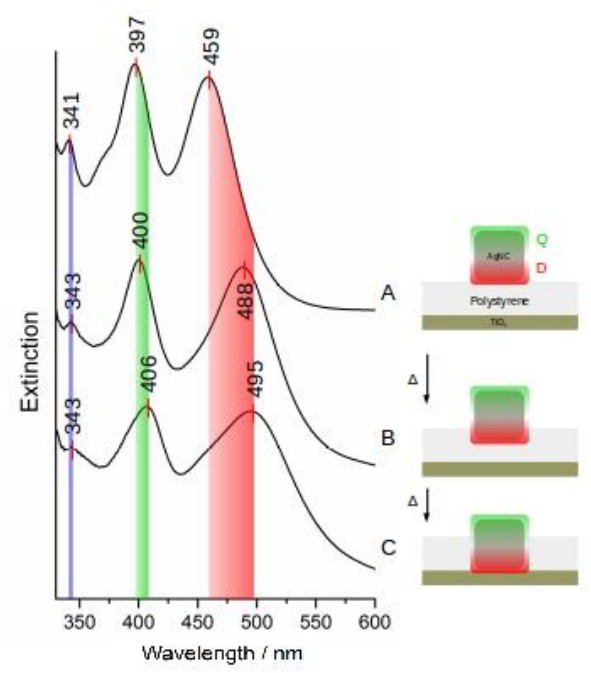

Figure 7.9. Experimental schematic: A monolayer of silver nanocubes deposited via the LangmuirSchaefer technique onto a thin $50 \mathrm{~nm}$ film of polystyrene on a $20 \mathrm{~nm}$ thin film of high refractive index titanium oxide. As the system is heated near the glass transition temperature of the underlying polymer the nanocubes begin to diffuse into the polymer layer. The position of the nanocubes and corresponding extinction spectrum are shown for the initial (A), intermediate (B), and final (C) position. Due to the high refractive index of the underlying titanium oxide, the $D$ mode shifts further when compared to polystyrene on glass system. The $\mathrm{Q}$ mode only shifts marginally as the cubes themselves are larger than the polymer layer and a large portion of the cube remains exposed keeping the $\mathrm{Q}$ mode sensitive to the surrounding environment (air) above the composite material.

\subsection{Conclusions}

We fabricated a nanocomposite composed of silver nanocube monolayers on polystyrene thin films as a platform for the precise control and observation of nanoparticles embedding into a polymer. This platform is a useful avenue for the investigation of surface energies and interfacial energies between surface passivated nanocrystals and polymer matrices, yielding insight into the behaviour and driving forces acting on the particles. We have also determined that once a system is carefully calibrated it becomes possible to know the average distance and orientation of the nanocrystals embedded into the polymer using simple far field optical measurements alone. This type of anisotropic nanoruler is based on 
plasmon hybridization induced by proximity to a dielectric substrate and utilizes the combined refractive index sensitivity of the spatially resolved plasmon modes. Contrary to a system based on interparticle and substrate-particle coupling modes or particle-emitter combinations, the use of hybrid modes to determine the anisotropy of the local environment is a simple platform utilizing the inherent properties of the particles while yielding a reasonably large quasilinear dynamic range similar to the size of the particle itself. The platform described above is a general robust approach to nano-positioning and sensing and can be easily modified to include additional parameters such as a high refractive index substrate below the polymer-nanocrystal composite.

\subsection{References}

1 C. Noguez, J. Phys. Chem. C, 2007, 111, 3806-3819.

2 A. Ianoul and A. Bergeron, Langmuir, 2006, 22, 10217-10222.

$3 \quad$ P. C. Lee and D. Meisel, J. Phys. Chem., 1982, 86, 3391-3395.

4 H. Robatjazi, S. M. Bahauddin, C. Doiron and I. Thomann, Nano Lett., 2015, 15, 6155-6161.

5 J. R. Adleman, D. A. Boyd, D. G. Goodwin and D. Psaltis, Nano Lett., 2009, 9, 44174423.

6 A. D. McFarland and R. P. Van Duyne, Nano Lett., 2003, 3, 1057-1062.

$7 \quad$ H. Tan, R. Santbergen, A. H. M. Smets and M. Zeman, Nano Lett., 2012, 12, 40704076.

8 J. Butet and O. J. F. Martin, ACS Nano, 2014, 8, 4931-4939.

$9 \quad$ S. Shen, L.-Y. Meng, Y. Zhang, J. Han, Z. Ma, S. Hu, Y. He, J.-F. Li, B. Ren, T.-M. Shih, Z. Wang, Z.-L. Yang and Z.-Q. Tian, Nano Lett., 2015, 15, 6716-6721.

10 R. E. Armstrong, R. A. Riskowski and G. F. Strouse, Photochem. Photobiol., 2015, 91, 732-738.

11 X. Wang, S. Li, P. Zhang, F. Lv, L. Liu, L. Li and S. Wang, Adv. Mater., 2015, 27, 6040-6045. 
12 R. T. Hill, K. M. Kozek, A. Hucknall, D. R. Smith and A. Chilkoti, ACS Photonics, 2014, 1, 974-984.

13 B. Gallinet, T. Siegfried, H. Sigg, P. Nordlander and O. J. F. Martin, Nano Lett., 2013, 13, 497-503.

14 R. T. Hill, J. J. Mock, A. Hucknall, S. D. Wolter, N. M. Jokerst, D. R. Smith and A. Chilkoti, ACS Nano, 2012, 6, 9237-9246.

15 G. Kovacs and P. Vincett, J. Colloid Interface Sci., 1982, 90, 335-351.

16 G. J. Kovacs and P. S. Vincett, Thin Solid Films, 1984, 111, 65-81.

17 R. D. Deshmukh and R. J. Composto, Langmuir, 2007, 23, 13169-13173.

18 J. Erichsen, J. Kanzow, U. Schürmann, K. Dolgner, K. Günther-Schade, T. Strunskus, V. Zaporojtchenko and F. Faupel, Macromolecules, 2004, 37, 1831-1838.

19 S. A. Hutcheson and G. B. McKenna, Phys. Rev. Lett., 2005, 94, 076103.

20 J. Teichroeb and J. Forrest, Phys. Rev. Lett., 2003, 91, 016104.

21 J. S. Sharp, J. H. Teichroeb and J. A. Forrest, Eur. Phys. J. E, 2004, 15, 473-487.

22 G. J. Kovacs, J. Vac. Sci. Technol., 1982, 20, 419.

23 P. K. Jain, W. Huang and M. A. El-Sayed, Nano Lett., 2007, 7, 2080-2088.

24 A. I. Dolinnyi, J. Phys. Chem. C, 2015, 119, 4990-5001.

25 N. Liu, M. Hentschel, T. Weiss, A. P. Alivisatos and H. Giessen, Science, 2011, 332, 1407-1410.

26 A. Moreau, C. Ciracì, J. J. Mock, R. T. Hill, Q. Wang, B. J. Wiley, A. Chilkoti and D. R. Smith, Nature, 2012, 492, 86-89.

2727 A. K. Singh, S. A. Khan, Z. Fan, T. Demeritte, D. Senapati, R. Kanchanapally and P. C. Ray, J. Am. Chem. Soc., 2012, 134, 8662-8669.

2828 C. J. Breshike, R. A. Riskowski and G. F. Strouse, J. Phys. Chem. C, 2013, 117, 23942-23949.

29 X. Zhang, C. A. Marocico, M. Lunz, V. A. Gerard, Y. K. Gun’ko, V. Lesnyak, N. Gaponik, A. S. Susha, A. L. Rogach and A. L. Bradley, ACS Nano, 2012, 6, 92839290. 
30 G. R. Bourret, T. Ozel, M. Blaber, C. M. Shade, G. C. Schatz and C. A. Mirkin, Nano Lett., 2013, 13, 2270-2275.

31 L. Li, T. Hutter, W. Li and S. Mahajan, J. Phys. Chem. Lett., 2015, 6, 2282-2286.

32 S. Zhang, K. Bao, N. J. Halas, H. Xu and P. Nordlander, Nano Lett., 2011, 11, 16571663.

33 N. Ahamad, A. Bottomley and A. Ianoul, J. Phys. Chem. C, 2012, 116, 185-192.

34 S. E. Skrabalak, L. Au, X. Li and Y. Xia, Nat. Protoc., 2007, 2, 2182-2190.

35 E. D. Palik, Handbook of Optical Constants, Academic Press, San Diego, 1998.

36 H. U. Yang, J. D’Archangel, M. L. Sundheimer, E. Tucker, G. D. Boreman and M. B. Raschke, Phys. Rev. B: Condens. Matter, 2015, 91, 235137.

37 T. A. F. König, P. A. Ledin, J. Kerszulis, M. A. Mahmoud, M. A. El-Sayed, J. R. Reynolds and V. V. Tsukruk, ACS Nano, 2014, 8, 6182-6192.

38 N. Sultanova, S. Kasarova and I. Nikolov, Acta Phys. Pol., A, 2009, 116, 585-587.

39 L. J. Sherry, S.-H. Chang, G. C. Schatz and R. P. Van Duyne, Nano Lett., 2005, 5, 2034-2038.

40 Q. Zhang, W. Li, C. Moran, J. Zeng, J. Chen, L. P. Wen and Y. Xia, J. Am. Chem. Soc., 2010, 132, 11372-11378.

41 N. Ahamad and A. Ianoul, J. Phys. Chem. C, 2011, 115, 3587-3594.

42 M. Mahmoud, C. Tabor and M. El-Sayed, J. Phys. Chem. C, 2009, 113, 5493-5501.

43 E. Ringe, J. M. McMahon, K. Sohn, C. Cobley, Y. Xia, J. Huang, G. C. Schatz, L. D. Marks and R. P. Van Duyne, J. Phys. Chem. C, 2010, 114, 12511-12516.

44 O. Nicoletti, F. De la Peña, R. K. Leary, D. J. Holland, C. Ducati and P. A. Midgley, Nature, 2013, 502, 80-84.

45 M. Bosman, V. J. Keast, M. Watanabe, A. I. Maaroof and M. B. Cortie, Nanotechnology, 2007, 18, 165505.

46 S. Mazzucco, N. Geuquet, J. Ye, O. Stéphan, W. Van Roy, P. Van Dorpe, L. Henrard and M. Kociak, Nano Lett., 2012, 12, 1288-1294.

47 D. Rossouw, M. Couillard, J. Vickery, E. Kumacheva and G. A. Botton, Nano Lett., 2011, 11, 1499-1504. 
48 E. J. R. Vesseur, R. de Waele, M. Kuttge and A. Polman, Nano Lett., 2007, 7, $2843-$ 2846.

49 J. Rieger, J. Therm. Anal., 1996, 46, 965-972.

50 G. Vignaud, M. S. Chebil, J. K. Bal, N. Delorme, T. Beuvier, Y. Grohens and A. Gibaud, Langmuir, 2014, 30, 11599-11608.

51 A. Kyrychenko, O. M. Korsun, I. I. Gubin, S. M. Kovalenko and O. N. Kalugin, J. Phys. Chem. C, 2015, 119, 7888-7899.

52 B. Gao, G. Arya and A. R. Tao, Nat. Nanotechnol., 2012, 7, 433-437.

53 J. Brandrup, E. H. Immergut and E. A. Grulke, Polymer Handbook, Wiley, New York, 4th edn, 1999.

54 M. K. Chaudhury, Mater. Sci. Eng., R, 1996, 16, 97-159.

55 T. Higashihara and M. Ueda, Macromolecules, 2015, 48, 1915-1929. 


\section{Concluding Remarks}

Plasmonics is a far reaching subject with potential applications across the sciences and engineering. Silver is one of the most studied plasmonic materials to date due to its unparalleled quality factor, ease of fabrication, and wide array of accessible shapes.

The major focus of this work was on the, understanding, optimization, and utilization of spatially separated hybrid plasmon modes present in ensembles of solid supported silver nanocubes. The work reported in this thesis reveals that the highly tunable nature of plasmonic modes present in solid supported silver nanocubes can be effectively extended to large ensembles in the macroscale, allowing for fabrication of optical materials with coherent predicitable behavour. The refractive index sensitivities and figures of merit for individual modes in these ensembles have shown that this platform can be utilized as highly effective RIS and SERS sensing platforms across the visible spectrum. Further more, the anisotropic behaviour exhibited by the hybrid modes in terms of scattering, absorption and transmission, with respect to illumination geometry extends both the understanding of these materials, but also their potential applications highlighted in chapter 6, and can be exploited for projection display technologies. ${ }^{1}$ This work also demonstrates the potential that can be gained from utilizing the spatial and energetic separation of all of the visible modes present in supported nanocrystals. This is highlighted in chapter 7 by utilization of modes as a collective set of nanorulers. This has potential for investigation and sensing of various interfacial systems, but also yields an easy far-field method for quality control and fine tuning of desired optical properties for nanocomposites that these particles.

Despite the large amount of fundamental research that has already been published on the subject of silver nanocrystals and their interactions with solid substrates, it is the opinion 
of the author that there is still a great deal of research that can be performed. Continued research on this topic could include directions such as utilizing thermoplasmonic properties as a method for imbedding a particle within a matrix such as a polymer, or as a method for triggering localized heating in thermochromic materials. Creative approaches utilizing the understanding of this system provided by this work will contiune to open up new avenues of application and fundamental science.

Some preliminary experiments performed by the author not included in this thesis, have potential in the utilization of atomic layer deposition of metal oxides to precisely control the effective refractive index of the superstrate. Precise control of both the substrate and superstrate appear to lead to super absorbers that are tunable in the visible spectrum. This in turn has potential for use in thermoplasmonic applications, RI sensing, field enhanced spectroscopy, and optical materials. ${ }^{2,3}$ Additionally, if one prepares monolayers of AgNCs on a substrate that inhibits ALD growth, it becomes possible to re-disperse the particles after the ALD process yielding a solution of cubes with an anisotropic shells leaving a single exposed face. This platform maintains the anisotropic in the local environment while in solution, additionally the cube can undergo galvanic exchange to produce a solid supported gold nanocage. ${ }^{4}$ A potential application of this could be plasmon enhanced photocatalysis. ${ }^{5}$

The majority of the experiments I performed involved the use of a dielectric substrate. However some experiments were performed on the generation of a coupling band between silver nanocubes and a thin $\sim 100 \mathrm{~nm}$ film of gold. The interaction was mediated by varying the gap between metals using polyelectrolyte layering. Details can be found in the SPIE proceedings listed below.

Research on silver nanowire coatings on optical fibers containing a tilted fiber Bragg 
grating was performed in collaboration with the Albert Group in the department of electronics at Carleton University, under the NSERC funded project 'Multimodal Optical Sensor Applications, Interfaces, and Controls’ (MOSAIC). The fiber grating scatters light out of the core of the fiber into the cladding, where it can intact with the surface and local environment around the fiber. My role in this project was the synthesis of silver nanowires, and self assembly of them over top of the region of the fiber that contains the grating. This lead to a 3.5x improvement in the refractive index sensitivity compared to the bare fiber. The details of this were produced an article, a proceedings paper, and a US patent.

Collaboration with the Ye group in the department of electronics at Carleton University, yielded progress and insight into the use of silver nanocubes for thin-film photovoltaic enhancement. The details of which were published in two proceedings papers for Photonics West.

\section{Patents, Articles, and Proceedings not included in this thesis:}

J. Albert, A. Ianoul, A. Bialiayeu, A. Bottomley, “Optical Fiber with Grating and Particulate Coating”, US Patent, 20150140556

A. Bialiayeu, A. Bottomley, D. Prezgot, A. Ianoul and J. Albert, "Plasmon-enhanced refractometry using silver nanowire coatings on tilted fibre Bragg gratings", Nanotechnology, 2012, 23, 444012

A. Bottomley and A. Ianoul, "Plasmonic properties of silver nanocube monolayers deposited on thin metal films”, SPIE OPTO, 2014, 899613-899613-6

F. Hejazi, S. Y. Ding, Y. Sun, A. Bottomley, A. Ianoul and W. N. Ye, "Improving photovoltaic devices using silver nanocubes”, SPIE OPTO, 2013, 862004-862004-9

A. Bottomley, A. Staff, D. Prezgot and A. Ianoul, "Plasmonic properties of weakly interacting silver nanocubes on high refractive index substrates”, SPIE BiOS, 2013, 859709859709-6

F. Hejazi, S. Y. Ding, Y. Sun, A. Bottomley, A. Ianoul and W. N. Ye, "Design of plasmonic enhanced silicon-based solar cells”, Photonics North, 2012, 84121L-84121L-7162

A. Bialiayeu, J. Albert, A. Ianoul, A. Bottomley and D. Prezgot, "Silver Nanowire Coated Tilted Fibre Bragg Gratings”, Advanced Photonics Congress, 2012, BW2E.1 


\section{Conference Presentations Given by the Author:}

Canadian Society for Chemistry

Canadian Society for Chemistry

Surface Plasmon Photonics 6

Photonics West

Photonics West

Canadian Society for Chemistry

Canadian Society for Chemistry

$\begin{array}{lr}\text { Ottawa, ON, CA } & \text { Oral }-2015 \\ \text { Vancouver, BC, CA } & \text { Oral }-2014 \\ \text { Ottawa, ON, CA } & \text { Poster }-2014 \\ \text { San Francisco, CA, US } & \text { Poster }-2014 \\ \text { San Francisco, CA, US } & \text { Oral }-2013 \\ \text { Quebec City, QC, CA } & \text { Oral }-2013 \\ \text { Calgary, AB, CA } & \text { Oral }-2012\end{array}$

Oral - 2014

Oral - 2013

Oral - 2012

\section{References}

(1) Saito, K.; Tatsuma, T. Nanoscale 2015, 7 (48), 20365-20368.

(2) Coppens, Z. J.; Li, W.; Walker, D. G.; Valentine, J. G. Nano Lett. 2013, 13 (3), 10231028.

(3) Grigoriev, V.; Bonod, N.; Wenger, J.; Stout, B. ACS Photonics 2015, 2 (2), 263-270.

(4) Skrabalak, S. E.; Au, L.; Li, X.; Xia, Y. Nat. Protoc. 2007, 2 (9), 2182-2190.

(5) Robatjazi, H.; Bahauddin, S. M.; Doiron, C.; Thomann, I. Nano Lett. 2015, 15 (9), 6155-6161. 